\title{
Segmentação de Objetos via Transformada Imagem-Floresta Orientada com Restrições de Conexidade
}

\author{
Lucy Alsina Choque Mansilla
}

TESE APRESENTADA

$\mathrm{AO}$

Instituto De Matemática e EstatísticA

DA

Universidade De SÃo Paulo

PARA

OBTENÇÃO DO TÍTULO

$\mathrm{DE}$

DOUTORA EM CiÊnCIAS

\author{
Programa: Ciência da Computação \\ Orientador: Prof. Dr. Paulo André Vechiatto de Miranda
}

Durante o desenvolvimento deste trabalho o autor recebeu auxílio financeiro da CAPES.

São Paulo, Agosto de 2018 


\title{
Segmentação de Objetos via Transformada Imagem-Floresta Orientada com Restrições de Conexidade
}

\author{
Esta versão da tese contém as correções e alterações sugeridas \\ pela Comissão Julgadora durante a defesa da versão original do trabalho, \\ realizada em 10/08/2018. Uma cópia da versão original está disponível no \\ Instituto de Matemática e Estatística da Universidade de São Paulo.
}

Comissão Julgadora:

- Prof. Dr. Paulo André Vechiatto de Miranda (orientador) - IME-USP

- Prof. Dr. Alexandre Xavier Falcão - UNICAMP

- Prof. Dr. Fábio Augusto Menocci Cappabianco - UNIFESP

- Prof. Dr. Thiago Vallin Spina - CNPEM

- Profa. Dra. Nina Sumiko Tomita Hirata - IME-USP 


\section{Agradecimentos}

Agradeço a Deus pela proteção, guia, sabedoria e por colocar as pessoas certas na minha vida e a minha família pelo grande amor, apoio, compreensão e incentivo.

Agradeço profundamente ao meu orientador, o professor Paulo André Vechiatto de Miranda pela disposição e orientação durante todo o meu doutorado.

Gostaria de agradecer aos professores Alexandre Falcão, Fábio Cappabianco, Thiago Spina e a professora Nina Hirata, pelas sugestões e correções dadas na defesa da tese, bem como aos professores Ronaldo Hashimoto e Marcos Tsuzuki pelas sugestões dadas no exame de qualificação. As idéias fornecidas foram fundamentais para a conclusão deste trabalho.

Também gostaria de agradecer aos meus colegas e amigos, obrigada pela amizade, apoio e brincadeiras. Fica na minha mente os momentos compartilhados que fizeram nossos dias mais alegres.

Finalmente, agradeço à CAPES pelo apoio financeiro durante meu doutorado, o que permitiu minha dedicação integral nesta pesquisa. 


\section{Resumo}

\section{CHOQUE-MANSILLA, LUCY A. Segmentação de Objetos via Transformada Imagem-}

Floresta Orientada com Restrições de Conexidade. 2018. 115 f. Tese - Instituto de Matemática e Estatística, Universidade de São Paulo, São Paulo, 2018.

Segmentação de objetos em imagens é um dos problemas mais fundamentais e desafiadores em processamento de imagem e visão computacional. O conhecimento de alto nível e específico do usuário é frequentemente requerido no processo de segmentação, devido à presença de fundos heterogêneos, objetos com bordas fracamente definidas, inomogeneidade de campo, ruído, artefatos, efeitos de volume parcial e seus efeitos conjuntos. Propriedades globais do objeto de interesse, tais como conexidade, restrições de forma e polaridade de borda, são conhecimentos prévios de alto nível úteis para a sua segmentação, permitindo a customização da segmentação para um objeto alvo.

Nesse trabalho, apresentamos um novo método chamado Transformada Imagem-Floresta Orientada Conexa (COIFT, Connected Oriented Image Foresting Transform), que fornece soluções ótimas globais de acordo com uma medida de corte em grafo, incorporando a restrição de conexidade na Transformada Imagem-Floresta Orientada (OIFT, Oriented Image Foresting Transform), com o fim de garantir a geração de objetos conexos, bem como permitir o controle simultâneo da polaridade de borda. Enquanto o emprego de restrições de conexidade em outros arcabouços, tais como no algoritmo de corte-mínimo/fluxo-máximo (min-cut/max-flow), leva a um problema NP-difícil, a COIFT conserva o baixo custo computacional da OIFT. Experimentos mostram que a COIFT pode melhorar consideravelmente a segmentação de objetos com partes finas e alongadas, para o mesmo número de sementes em segmentação baseada em marcadores.

Palavras-chave: transformada imagem-floresta, transformada imagem-floresta orientada, restrição de conexidade, segmentação de imagens, segmentação por corte em grafo, funções de conexidade monotonicamente incrementais, funções de conexidade não monotonicamente incrementais. 


\section{Abstract}

\section{CHOQUE-MANSILLA, LUCY A. Object Segmentation by Oriented Image Foresting}

Transform with Connectivity Constraints. 2018. 115 f. Thesis - Institute of Mathematics and Statistics University of São Paulo, São Paulo, 2018.

Object segmentation is one of the most fundamental and challenging problems in image processing and computer vision. The high-level and specific knowledge of the user is often required in the segmentation process, due to the presence of heterogeneous backgrounds, objects with poorly defined boundaries, field inhomogeneity, noise, artifacts, partial volume effects and their joint effects. Global properties of the object of interest, such as connectivity, shape constraints and boundary polarity, are useful high-level priors for its segmentation, allowing the customization of the segmentation for a given target object.

In this work, we introduce a new method called Connected Oriented Image Foresting Transform (COIFT), which provides global optimal solutions according to a graph-cut measure in graphs, subject to the connectivity constraint in the Oriented Image Foresting Transform (OIFT), in order to ensure the generation of connected objects, as well as allowing the simultaneous control of the boundary polarity. While the use of connectivity constraints in other frameworks, such as in the min-cut/max-flow algorithm, leads to a NP-Hard problem, COIFT retains the low computational cost of OIFT. Experiments show that COIFT can considerably improve the segmentation of objects with thin and elongated parts, for the same number of seeds in segmentation based on markers.

Keywords: image foresting transform, oriented image foresting transform, connectivity constraints, image segmentation, graph-cut segmentation, monotonic-incremental path-cost functions, non-monotonically-incremental path-cost functions. 


\section{Sumário}

Lista de Abreviaturas $\quad$ xi

Lista de Símbolos $\quad$ xiii

Lista de Figuras $\quad$ Xv

$\begin{array}{lc}\text { Lista de Tabelas } & \text { xvii }\end{array}$

1 Introdução $\quad 1$

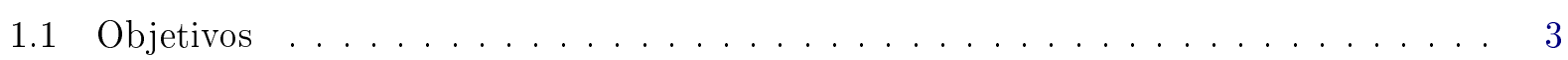

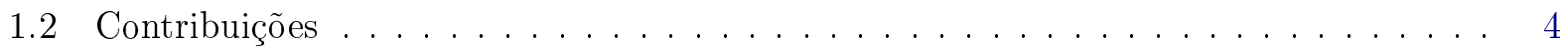

1.3 Organização do Trabalho . . . . . . . . . . . . . . . . . . . 4

2 Conceitos 5

2.1 Noções de imagem digital . . . . . . . . . . . . . . . . . . . . . . . . 5

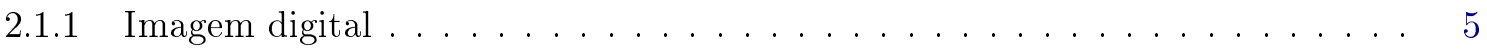

2.1 .2 Representação de uma imagem . . . . . . . . . . . . . . . . . . 6

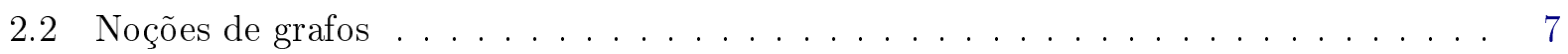

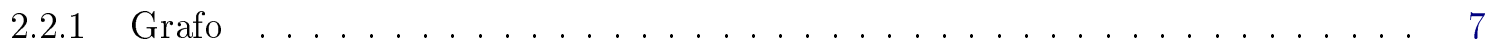

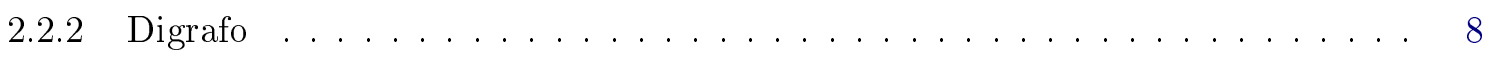

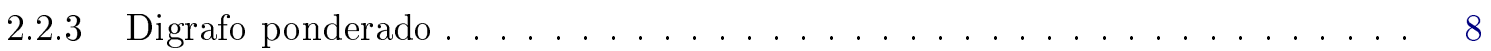

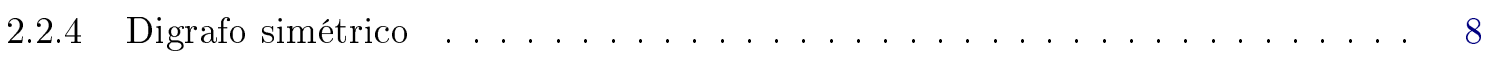

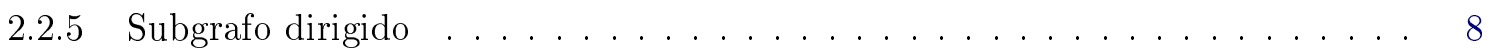

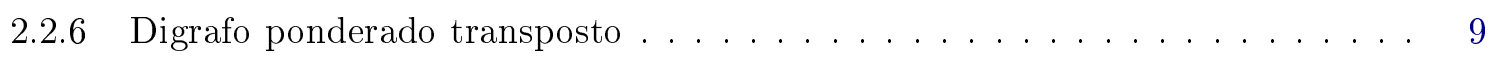

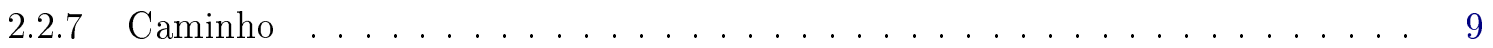

2.2 .8 Ciclo . . . . . . . . . . . . . . . . . . . . . 10

2.2.9 Digrafo ponderado fortemente conexo . . . . . . . . . . . . . . . . . 10

2.3 Grafos a partir de imagens . . . . . . . . . . . . . . . . . . . . . 10

2.4 Segmentação de objetos no digrafo da imagem . . . . . . . . . . . . . . . . . 13

2.4 .1 Segmentação binária . . . . . . . . . . . . . . . . . . . 13

2.4 .2 Segmentação com sementes . . . . . . . . . . . . . . . . . . . . . . 14

2.5 Avaliação do desempenho dos métodos de segmentação . . . . . . . . . . . . . . . . . . . . .

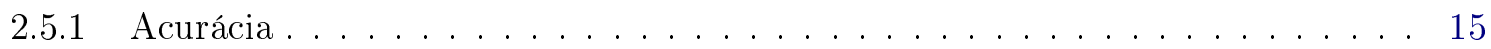

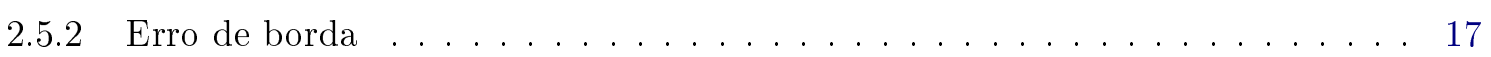


3 Métodos relacionados $\quad 21$

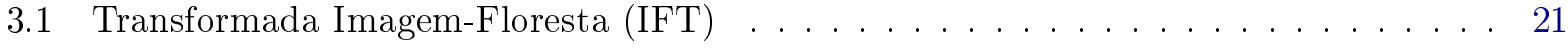

3.1 .1 Definição . . . . . . . . . . . . . . . . . . . . . . . . . 21

3.1 .2 Função de custo de caminho não MI . . . . . . . . . . . . . . . . . . . 24

3.2 Segmentação por competição de sementes . . . . . . . . . . . . . . . . 25

3.2 .1 Exemplo de segmentação . . . . . . . . . . . . . . . . . . . 25

3.2 .2 Algoritmo da IFT . . . . . . . . . . . . . . . . . . . . . . 27

3.2 .3 Fila de prioridade . . . . . . . . . . . . . . . . . . 27

3.2 .4 Zonas de empate . . . . . . . . . . . . . . . . . . . 28

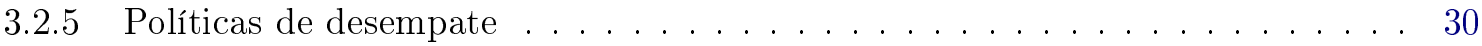

3.3 Condições suficientes para garantir a otimalidade dos caminhos da IFT . . . . . . . . 32

3.3.1 Contra-exemplo para as condições de suavidade da IFT original (PAMI, 2004) 32

3.3.2 Versão suficiente das condições (ISMM, 2017) . . . . . . . . . . . . . 35

3.4 Corte em grafo generalizado . . . . . . . . . . . . . . . . . 36

3.4 .1 Funções de energia . . . . . . . . . . . . . . . . . . . . 36

3.5 Transformada Imagem-Floresta Orientada $(\mathrm{OIFT}) \ldots \ldots \ldots \ldots$. . . . . . . 38

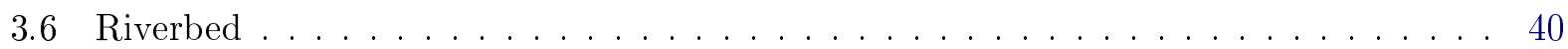

4 Transformada Imagem-Floresta Orientada com restrições de conexidade 41

4.1 Restrição de conexidade . . . . . . . . . . . . . . . . . . . . . . . . . 41

4.2 Segmentação de um objeto conexo via COIFT . . . . . . . . . . . . . . . . . 43

4.2 .1 Algoritmo da COIFT . . . . . . . . . . . . . . . . . . . 46

4.3 Segmentação de um objeto $\gamma$-conexo via COIFT . . . . . . . . . . . . . . . . 47

4.3.1 Algoritmo da COIFT com largura ajustável . . . . . . . . . . . . . . . . . 49

4.3.2 Tratamento de empates de energia . . . . . . . . . . . . . . . . 53

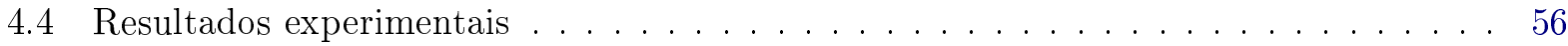

4.4 .1 Conjuntos de imagens . . . . . . . . . . . . . . . . . . . . . 56

4.4 .2 Seleção automática de marcadores . . . . . . . . . . . . . . . . . . . . 59

4.4 .3 Métodos comparados . . . . . . . . . . . . . . . . . . . . . . 60

4.4.4 Experimentos usando COIFT sem largura ajustável . . . . . . . . . . . . . . . 60

4.4.5 Experimentos usando COIFT com largura ajustável . . . . . . . . . . . . . 62

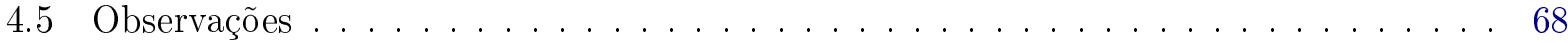

4.5.1 Restrições de conexidade para sementes do fundo . . . . . . . . . . . . 68

4.5.2 Casos intratáveis na COIFT . . . . . . . . . . . . . . . . . . . . . 69

5 Corte em grafo com funções não MI $\quad 71$

5.1 Corte ótimo com a função não MI $f_{\omega_{v}} \ldots \ldots \ldots \ldots \ldots \ldots \ldots \ldots \ldots$

6 Conclusões $\quad 73$

6.1 Considerações finais . . . . . . . . . . . . . . . . . . . . . 73

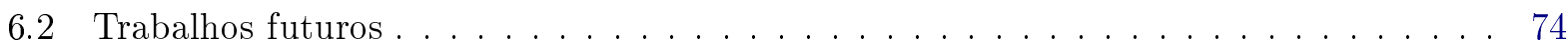

$\begin{array}{ll}\text { A Prova de corretude da Proposição } 1 & 75\end{array}$

$\begin{array}{ll}\text { B Prova de corretude da Proposição } 2 \text { da COIFT } & 77\end{array}$ 
D Prova do teorema da otimalidade do corte pela COIFT com largura ajustável

E Prova do teorema da otimalidade do corte da IFT com $f_{\omega_{v}}$ 


\section{Lista de Abreviaturas}

COIFT Transformada Imagem-Floresta Orientada Conexa (Connected Oriented Image Foresting Transform).

GC Corte em Grafo (Graph Cut).

GGC Corte em Grafo Generalizado (Generalized Graph Cut).

FNMI Funções de custo de caminho não monotonicamente incrementais.

IFT Transformada Imagem-Floresta (Image Foresting Transform).

IFT-SC IFT por Competição de Sementes ( IFT Segmentation by Seed Competition).

IRFC Conexidade Fuzzy Relativa Iterativa (Iterative Relative Fuzzy Connectedness).

MBD Distância mínima de barreira (Minimum Barrier Distance).

MI Monotonicamente incremental.

OIFT Transformada Imagem-Floresta Orientada (Oriented Image Foresting Transform).

ORFC Conexidade Fuzzy Relativa Orientada (Oriented Relative Fuzzy Connectedness).

PW Power Watersheds.

RM-T1 Ressonância Magnética 1 Tesla.

TC Tomografia Computadorizada. 


\section{Lista de Símbolos}

$\mathbb{R} \quad$ conjunto dos números reais.

$\mathbb{R}^{n} \quad$ conjunto dos números reais em $n$ dimensões.

$\mathbb{Z} \quad$ conjunto dos números inteiros.

$\mathbb{Z}^{n} \quad$ conjunto dos números inteiros em $n$ dimensões.

$\hat{I} \quad$ Imagem digital.

$\mathcal{I}$ Domínio da imagem, considerado também como o conjunto de vértices $\mathcal{V}$.

$G \quad$ Digrafo ponderado, simétrico, fortemente conexo.

$\mathcal{V} \quad$ Conjunto de vértices no digrafo $G$.

$\mathcal{A} \quad$ Conjunto de arcos no digrafo $G$.

$\langle s, t\rangle \quad$ Arco com origem no pixel $s$ e destino no pixel $t$.

$\omega_{a}, \delta_{a}, \psi_{a}$ Pesos associados aos arcos.

$\omega_{v} \quad$ Peso associado aos vértices.

$\alpha \quad$ Parâmetro do fator de orientação.

$I(t) \quad$ Intensidade do pixel $t$.

$f \quad$ Função de custo de caminho.

$\mathcal{O} \quad$ Objeto no digrafo da imagem.

$\mathcal{O}_{\text {opt }} \quad$ Objeto ótimo.

$\mathcal{O}^{\text {OIFT }}$ Objeto calculado pelo algoritmo da OIFT.

$\mathcal{O}^{\text {COIFT }}$ Objeto calculado pelo algoritmo da COIFT.

$\mathcal{S} \quad$ Conjunto de sementes.

$\mathcal{S}_{1} \quad$ Conjunto de sementes internas que representam o objeto na imagem.

$\mathcal{S}_{\mathbf{0}} \quad$ Conjunto de sementes externas que representam o fundo na imagem.

$\mathcal{S}_{1}^{c} \quad$ Conjunto de sementes internas conexas.

$\mathcal{S}_{1}^{\gamma c} \quad$ Conjunto de sementes internas $\gamma$-conexas.

$\pi \quad$ Caminho.

П $\quad$ Família de caminhos.

$\pi_{s} \quad$ Caminho com destino no pixel $s$.

$\pi_{s \sim t} \quad$ Caminho com origem no pixel $s$ e destino no pixel $t$.

$\pi_{\mathcal{S} \sim t} \quad$ Caminho com origem em um pixel pertencente ao conjunto de sementes $\mathcal{S}$ e destino no pixel $t$.

$\pi_{s}^{P} \quad$ Caminho definido por uma floresta de espalhamento $P$, com destino no pixel $s$. 
$\hat{L} \quad$ Imagem de rótulos.

$L(s) \quad$ Rótulo do pixel $s$ na imagem de rótulos $\hat{L}$.

$V, V_{\text {opt }}^{f} \quad$ Mapas de custos.

$\mathcal{Q} \quad$ Fila de prioridade.

$\mathcal{C}(\mathcal{O}) \quad$ Borda de corte do objeto $\mathcal{O}$.

$\mathcal{C}\left(\mathcal{O}_{\text {opt }}\right)$ Borda de corte do objeto ótimo $\mathcal{O}_{\text {opt }}$.

$E, \varepsilon_{1}, \varepsilon_{\infty} \quad$ Funções de energia da borda de corte de um objeto na imagem.

$E_{\{\boldsymbol{t}\}} \quad$ Energia ótima a partir de uma única semente interna $t$.

$E_{\boldsymbol{A}} \quad$ Energia ótima a partir de um conjunto de sementes internas $\boldsymbol{A}$. 


\section{Lista de Figuras}

1.1 Exemplo de classificação dos métodos de segmentação segundo o nível de conexidade 3

2.1 Exemplo do mapeamento entre coordenadas espaciais e paramétricas . . . . . . . . . 6

2.2 Representação de uma imagem . . . . . . . . . . . . . . . . . . . . . . . . . 7

2.3 Exemplo de representação de um grafo . . . . . . . . . . . . . . . . . . . . 7

2.4 Exemplo de representação de um digrafo . . . . . . . . . . . . . . . . . . 8

2.5 Exemplo de representação de arcos antiparalelos . . . . . . . . . . . . . . . 8

2.6 Exemplo de caminho em um digrafo de vizinhança-4 . . . . . . . . . . . . . . . 9

2.7 Exemplo de adjacências Euclidianas . . . . . . . . . . . . . . . . . . . . . . . . 11

2.8 Exemplo de adjacência assimétrica . . . . . . . . . . . . . . . . . . 11

2.9 Exemplo de digrafos gerados a partir de uma imagem 2D f . . . . . . . . . . . 12

2.10 Exemplo de segmentação binária . . . . . . . . . . . . . . . . . . . . . 13

2.11 Exemplo de segmentação satisfazendo as restrições de sementes . . . . . . . . . . . . 14

2.12 Diagrama da relação entre os conjuntos usados no Coeficiente de Dice . . . . . . . . 16

2.13 Exemplo de segmentação para um método específico, indicando os verdadeiros e falsos positivos, bem como os verdadeiros e falsos negativos . . . . . . . . . . . . 17

2.14 Exemplo comparativo do uso do Coeficiente de Dice e Erro de Borda . . . . . . . . . . 19

3.1 Exemplo de uma floresta de espalhamento na IFT . . . . . . . . . . . . . . . . . . 23

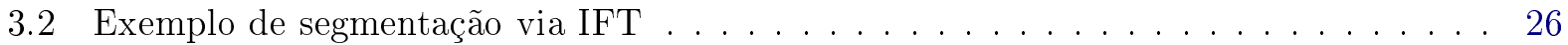

3.3 Exemplo de segmentação pela IFT com a função $f_{\max } \ldots \ldots \ldots \ldots$. . . . . . . 26

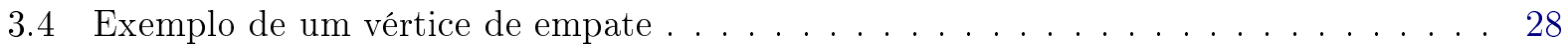

3.5 Exemplo de zonas de empate . . . . . . . . . . . . . . . . . . . . . . . 29

3.6 Exemplo de políticas de desempate para o problema de ambiguidade das florestas . . 31

3.7 Contra-exemplo das condições C1-C3 de uma função de conexidade suave na IFT . . 33

3.8 Contra-exemplo para a definição de suavidade da IFT, considerando $f_{C E}$ e o digrafo

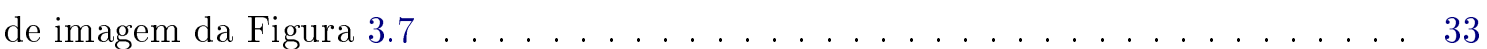

3.9 Contra-exemplo para a definição de suavidade da IFT, mostrando a floresta calculada

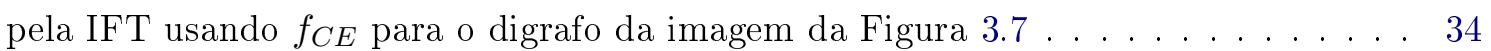

3.10 Exemplo de segmentação usando a função de energia $\varepsilon_{\infty}$ na sua forma dual . . . . . 37

3.11 Exemplo de segmentação com maximização da função de energia $E$. . . . . . . . . 37

3.12 Exemplo de segmentação via OIFT . . . . . . . . . . . . . . . . . . . . . . . . 39

3.13 Exemplo de segmentação por Riverbed . . . . . . . . . . . . . . . . . . . . . . . . . 40

4.1 Exemplo da importância do uso da restrição de conexidade . . . . . . . . . . . . . . . . 42 
4.2 Exemplo de objetos conexos e não conexos . . . . . . . . . . . . . . . . . 42

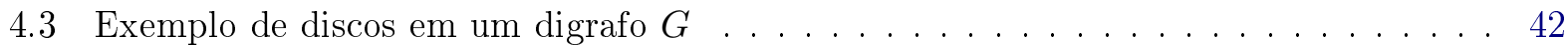

4.4 Exemplos de objetos $\gamma$-conexos e não $\gamma$-conexos $\ldots \ldots \ldots$. . . . . . . . . 43

4.5 Exemplo de um caminho usando a função $f_{\max }^{\# \mathcal{S}_{0}} \ldots \ldots \ldots \ldots \ldots \ldots \ldots \ldots$

4.6 Representação gráfica com resultados das segmentações por OIFT e COIFT . . . . 46

4.7 Exemplos de segmentações via COIFT com largura ajustável . . . . . . . . . . . . . 48

4.8 Representação gráfica com resultados de segmentações dadas pela OIFT e COIFT

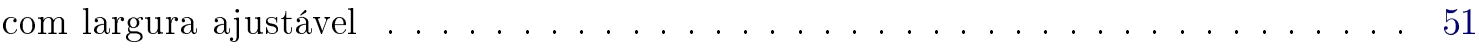

4.9 Exemplo de segmentação via OIFT e COIFT com largura ajustável . . . . . . . . . . 52

4.10 Representação esquemática da segmentação de uma imagem sintética pela OIFT e COIFT no caso de empates de energias . . . . . . . . . . . . . . . . 54

4.11 Exemplo de segmentação pela COIFT com largura ajustável considerando o tratamento de empates de energias . . . . . . . . . . . . . . . . 55

4.12 Exemplo de segmentação da vértebra da coluna. . . . . . . . . . . . . . . 56

4.13 Exemplo de segmentação da massa cinzenta. . . . . . . . . . . . . . . . . 57

4.14 Exemplo de segmentação de um pássaro. . . . . . . . . . . . . . . . . . . 5 57

4.15 Exemplo de segmentação de um pássaro. . . . . . . . . . . . . . . . . . 58

4.16 Exemplo de segmentação de um inseto. . . . . . . . . . . . . . . . . . 58

4.17 Exemplo dos conjuntos de sementes $\mathcal{S}_{\mathbf{1}}$ e $\mathcal{S}_{\mathbf{0}}$ calculados automaticamente . . . . . . 59

4.18 Exemplo de segmentação pela OIFT e COIFT . . . . . . . . . . . . . . . . . . 60

4.19 Exemplo de segmentação da vértebra da coluna via COIFT . . . . . . . . . . . . . 61

4.20 Curva de acurácia média da segmentação da vértebra da coluna por um usuário robô. 61

4.21 Segmentação da massa cinzenta cortical via OIFT e COIFT com largura ajustável 62

4.22 Curva de erro médio (erro de borda) usando um usuário robô para a segmentação da

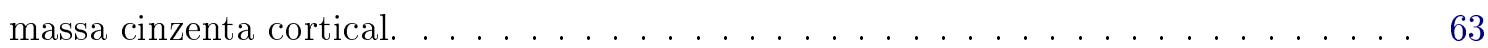

4.23 Segmentações de algumas imagens pertencentes ao conjunto de imagens dos pássaros via OIFT e COIFT com largura ajustável . . . . . . . . . . . . . . . . . . . 64

4.24 Curva de erro médio (erro de borda) usando um usuário robô para segmentar o conjunto de imagens dos pássaros (terceiro conjunto de imagens). . . . . . . . . . 65

4.25 Curva de erro médio (erro de borda) usando os extremos do esqueleto e erosão do objeto no gabarito para segmentar o conjunto de imagens dos pássaros (quarto conjunto

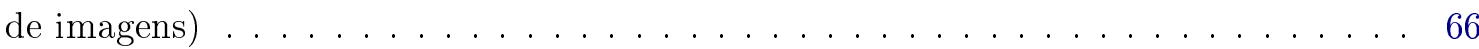

4.26 Curva de erro médio (erro de borda) usando os extremos do esqueleto e erosão do objeto no gabarito para segmentar o conjunto de imagens dos insetos $\ldots \ldots$. . . . 67

4.27 Exemplo de restrição de conexidade para sementes do fundo . . . . . . . . . . . . . 68

4.28 Exemplos de casos intratáveis via COIFT . . . . . . . . . . . . . . . . . . . 69

5.1 Exemplo da otimalidade do corte da IFT com a função $f_{\omega_{v}} \ldots \ldots \ldots \ldots \ldots$

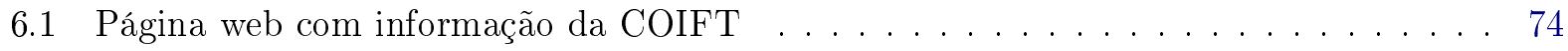

E.1 Representação esquemática para a prova de corretude do Teorema $4 \ldots \ldots$. . . . . 90 


\section{Lista de Tabelas}

2.1 Matriz de confusão para a segmentação binária . . . . . . . . . . . . . . . . . . 16 


\section{Capítulo 1}

\section{Introdução}

A segmentação de imagem é um dos problemas mais fundamentais e desafiadores em processamento de imagem e visão computacional. O conhecimento de alto nível e específico do domínio da aplicação do usuário é frequentemente requerido no processo da segmentação, devido à presença de fundos heterogêneos, objetos com bordas fracamente definidas, inomogeneidade de campo, ruído, artefatos, efeitos de volume parcial e seus efeitos conjuntos [Madabhushi e Udupa (2005)].

Matemática Discreta fornece uma estrutura elegante para o processamento de imagens, sendo rica em algoritmos eficientes, com provas de corretude. Como consequência, muitos métodos de segmentação de imagem foram modelados como problemas de busca e otimização em grafos [Couprie et al. (2010); Falcão et al. (1998, 2000); Grady (2006); Lézoray e Grady (2012); Peng et al. (2011); W.Yang et al. (2010)]. Em tais abordagens, uma importante classe de segmentação considera o problema de particionamento ótimo do grafo sujeito a restrições rígidas, conhecidas como sementes [Bai e Sapiro (2007); Boykov e Funka-Lea (2006); Ciesielski et al. (2007); Cousty et al. (2010); Grady (2006)]. Vários métodos dessa classe podem ser descritos aproximadamente em uma forma unificada de acordo com um arcabouço comum, às vezes referido como, Corte em Grafo Generalizado (GGC, Generalized Graph Cut) [Ciesielski et al. (2012b); Couprie et al. (2010)]. No contexto de segmentação binária, dentro desse arcabouço, existem duas classes importantes de formulações de energia, os problemas de minimização das energias $\varepsilon_{1}$ e $\varepsilon_{\infty}$, como discutido em [Ciesielski et al. (2012b)]. O problema da minimização de $\varepsilon_{1}$ inclui o algoritmo de corte-mínimo/fluxo-máximo (min-cut/max-flow), também popularmente conhecido como método de Corte em Grafo (GC, Graph Cut) [Boykov e Funka-Lea (2006)], enquanto que a segunda classe da minimização de $\varepsilon_{\infty}$ compreende métodos como Bacia Hidrográfica (Watersheds) [Cousty et al. (2010)], Conexidade Fuzzy (FC, Fuzzy Connectedness) [Ciesielski et al. (2007)] e alguns casos particulares da Transformada Imagem-Floresta (IFT, Image Foresting Transform) [Falcão et al. (2004)].

Propriedades globais, tais como conexidade, restrições de forma e polaridade de borda, são conhecimentos prévios de alto nível potencialmente úteis para a segmentação de objetos, permitindo a customização da segmentação para um dado objeto alvo [Lézoray e Grady (2012)]. A conexidade de um objeto na imagem é uma importante propriedade topológica global que foi alvo de estudo em diferentes arcabouços para segmentação de imagens [Lézoray e Grady (2012); Nowozin e Lampert (2010); Vicente et al. (2008); Zeng et al. (2008)].

Dada uma imagem binária representando o resultado de uma segmentação, nesse trabalho, consideramos um componente conexo como um conjunto maximal de pixels, tal que existem caminhos compostos por pixels vizinhos (exemplo: vizinhança-4 ou vizinhança-8) interligando todos seus ele- 
mentos e passando exclusivamente por dentro do objeto.

No contexto de segmentação binária, os métodos baseados em sementes podem ser classificados em três grupos, de acordo com seu nível de conexidade:

1. No primeiro grupo, temos métodos que não garantem nenhum nível de conexidade (Figuras 1.1b). Na comunidade do Corte em Grafo (GC), essa falta de conexidade é usualmente referida como o problema da desconexidade do GC, que ocorre quando os vértices da fonte e sorvedouro estão conectados a todos os pixels da imagem em um modelo de grafo estendido, porém isso resulta em segmentações que não possuem conexidade na topologia da imagem [Boykov e Funka-Lea (2006)].

2. No segundo grupo, nós temos métodos que garantem que os pixels do objeto são conexos a alguma semente interna. No entanto, note que o objeto poderia ser composto por vários componentes conexos separados, contanto que existam sementes do objeto marcadas em cada componente (Figura 1.1c). A maioria dos métodos de segmentação baseados em sementes pertencem a essa classe, incluindo os métodos de Conexidade Fuzzy e Watershed por Marcadores [Ciesielski et al. (2007); Cousty et al. (2010)].

3. No terceiro grupo, temos métodos que garantem que o objeto segmentado forma um único componente conexo no domínio da imagem [Lézoray e Grady (2012); Nowozin e Lampert (2010); Vicente et al. (2008); Zeng et al. (2008)]. Isso é especialmente importante quando o alvo é um objeto único (Figura 1.1d). 


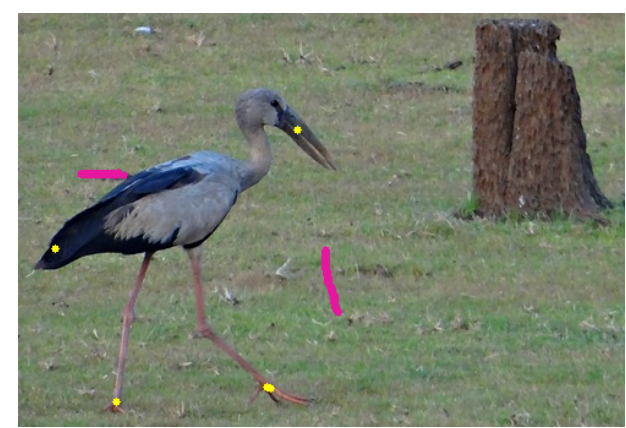

(a)

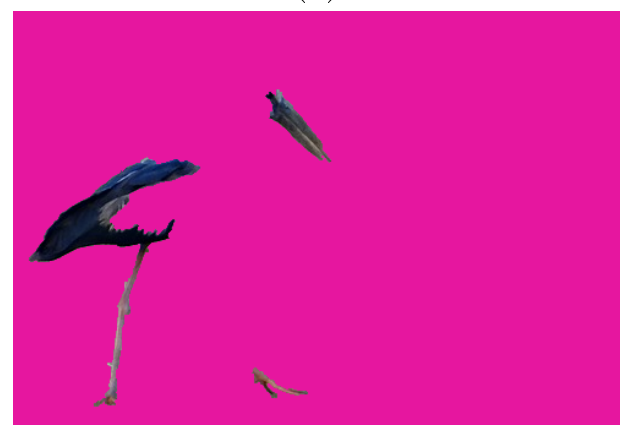

(c)

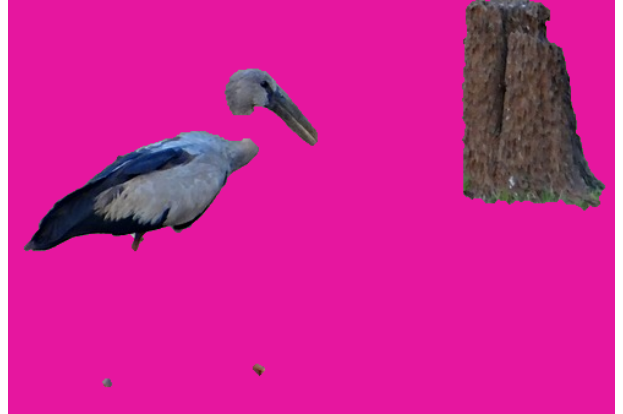

(b)

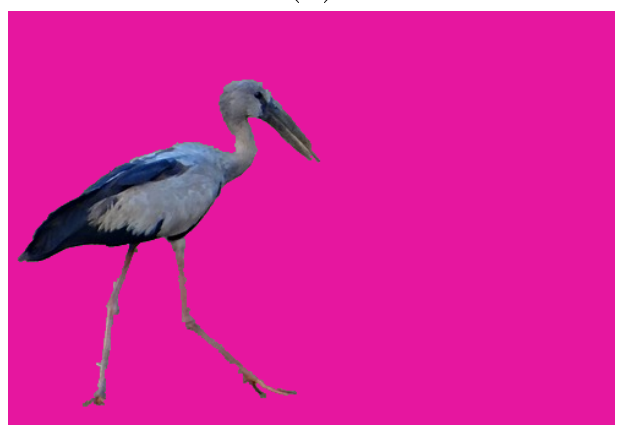

(d)

Figura 1.1: Exemplo de classificação dos métodos de segmentação segundo o nivel de conexidade. (a) Imagem de entrada com sementes selecionadas pelo usuário. (b) Segmentação por Corte em Grafo (GC) mostrando o problema da desconexidade de uma região do objeto à direita, que não foi marcada por nenhuma semente. (c) Segmentação pela IFT resultando em múltiplos componentes conexos, porém estando todos marcados por alguma semente do objeto. (d) Segmentação pelo método proposto produzindo um único componente conexo.

Neste trabalho, usamos o termo de restrição de conexidade para indicar métodos pertencentes ao terceiro grupo.

A minimização de energia $\varepsilon_{1}$ entre todos os objetos satisfazendo a restrição de conexidade foi provada ser um problema NP-difícil [Vicente et al. (2008); Zeng et al. (2008)]. Vicente et al. [Vicente et al. (2008)] propuseram um algoritmo heurístico, chamado DijkstraGC, o qual combina os algoritmos de Dijkstra e Corte em Grafo (GC). No entanto, DijkstraGC ainda é lento, devido ao fato de ele requerer muitas chamadas do algoritmo de corte-mínimo/fluxo-máximo (mincut/max-flow). Outro método, chamado Topology cuts, por Zeng et al. [Zeng et al. (2008)] também encontra somente uma solução aproximada para incorporar conhecimentos prévios da topologia no algoritmo de corte-mínimo/fluxo-máximo (min-cut/max-flow). Nowozin e Lampert adotaram um enfoque diferente resolvendo um problema relacionado de otimização, o qual força a conexidade da rotulagem no arcabouço de Máximo A Posteriori e Campo Aleatório de Markov (MAP-MRF, Maximum A Posteriori - Markov Random Field) [Lézoray e Grady (2012); Nowozin e Lampert (2010)].

\subsection{Objetivos}

Neste trabalho, novas extensões sobre segmentação de imagem usando grafos que exploram a Transformada Imagem-Floresta (IFT) [Falcão et al. (2004)] são propostas, motivadas pelos recentes avanços que têm sido feitos nessa classe, tal como a introdução de restrições de forma, 
incluindo a convexidade geodésica em estrela (GSC, Geodesic Star Convexity) [Mansilla et al. (2013a)] e a restrição de banda geodésica [de Moraes Braz e Miranda (2014)], e polaridade de borda [Mansilla et al. (2013b); Miranda e Mansilla (2014)]. Assim, nosso principal objetivo foi desenvolver um novo método de segmentação baseado em sementes que garanta resultados ótimos sujeitos à restrição de conexidade no problema da minimização de $\varepsilon_{\infty}$ do arcabouço do Corte em Grafo Generalizado (GGC).

\subsection{Contribuições}

Note que, sempre que o conjunto de sementes de objeto forma um único componente conexo no grafo, a segmentação resultante também será conexa para métodos do grupo 2. Portanto, uma forma de converter métodos do grupo 2 para o grupo 3, consiste em estender o conjunto de sementes de objeto para um conjunto conexo de sementes. Com base nesse princípio, propomos um novo método do grupo 3, porém selecionando dentre todas possíveis extensões das sementes de objeto, uma extensão ótima de acordo com uma energia de corte em grafo.

O novo algoritmo, chamado Transformada Imagem-Floresta Orientada Conexa (COIFT, Connected Oriented Image Foresting Transform) [Mansilla e Miranda (2016); Mansilla et al. (2016)], fornece soluções ótimas globais para o problema da minimização de $\varepsilon_{\infty}$ (o qual será apresentado aqui na sua forma dual equivalente, como um problema de maximização), sujeito a restrições de borda de alto nível (restrições de conexidade e polaridade), conservando a baixa complexidade computacional do algoritmo da IFT.

Uma segunda contribuição corresponde à prova da otimalidade, de acordo com uma medida de corte em grafo, de uma função não monotonicamente incremental, que corresponde a um caso particular da função de custo do Riverbed [Miranda et al. (2012)], em segmentação por região.

Por fim, apresentamos um estudo sobre condições suficientes para garantir a otimalidade dos caminhos calculados pela IFT [Condori et al. (2017)], demonstrando por meio de um contra-exemplo, a insuficiência das condições originalmente propostas por Falcão et al. [Falcão et al. (2004)].

\subsection{Organização do Trabalho}

No Capítulo 2 apresentamos os conceitos básicos e notações referentes a imagens digitais, teoria de grafos e segmentação de imagens, os quais serão usados nos capítulos seguintes. A metodologia adotada usa a IFT, a qual é apresentada no Capítulo 3. Nesse capítulo também são apresentados alguns outros métodos relacionados como Corte em Grafo Generalizado (GGC), Transformada Imagem-Floresta Orientada (OIFT, Oriented Image Foresting Transform) e o método Riverved [Miranda et al. (2012)], incluindo na Seção 3.3 uma revisão das condições de otimalidade da IFT conduzidas durante o doutorado. No Capítulo 4, o nosso novo método com restrição de conexidade é então apresentado. Outras contribuições realizadas durante o doutorado são apresentadas no Capítulo 5. Finalmente, no Capítulo 6 discutimos algumas conclusões sobre os resultados obtidos. As provas teóricas realizadas de proposições e teoremas do trabalho estão disponíveis nos Apêndices A-E . 


\section{Capítulo 2}

\section{Conceitos}

Neste capítulo são apresentados os conceitos básicos e notações que serão usados ao longo do documento. No entanto, leitores mais familiarizados podem ir diretamente para o Capítulo 3.

\subsection{Noções de imagem digital}

Seja $\hat{I}_{c}=\left\langle\mathcal{I}_{\boldsymbol{c}}, \vec{I}_{c}\right\rangle$ uma imagem contínua (real ou física), na qual $\mathcal{I}_{\boldsymbol{c}}$ representa o espaço da imagem em $\mathbb{R}^{n}$ (espaço contínuo) e $\vec{I}_{c}: \mathcal{I}_{\boldsymbol{c}} \rightarrow \mathbb{R}^{m}$ é uma função contínua que mapeia $\mathcal{I}_{\boldsymbol{c}}$ em um conjunto de $m$ propriedades físicas da imagem, como a reflexão de luz (brilho). Atualmente, os dispositivos de aquisição de imagens não conseguem gerar uma imagem contínua, pois computacionalmente é inviável mapear os infinitos valores de $\mathcal{I}_{\boldsymbol{c}}$ com os infinitos valores possíveis que as propriedades físicas da imagem podem assumir. Assim, uma conversão de uma imagem contínua $\hat{I}_{c}$ para uma imagem digital é necessária. Nessa conversão é necessário selecionar somente algumas amostras de $\mathcal{I}_{\boldsymbol{c}}$ (processo de amostragem) e associar cada amostra a um ou mais valores discretos representativos de alguma ou algumas propriedades físicas da imagem (processo de quantização).

\subsubsection{Imagem digital}

Seja $\hat{I}=\langle\mathcal{I}, \vec{I}\rangle$ uma imagem digital, na qual $\mathcal{I}$ é o domínio da imagem representado por um conjunto finito de pontos do $\mathbb{Z}^{n}$, cujos elementos são conhecidos como spels (space elements), e $\vec{I}: \mathcal{I} \rightarrow \mathbb{Z}^{m}$ é um mapeamento vetorial que associa a cada spel $t \in \mathcal{I}$ um conjunto de $m$ valores escalares $I_{i}(t)$, com $i=1,2, \ldots, m$, os quais estão associados com alguma propriedade física. $\mathrm{O}$ valor de $n$ refere-se à dimensão da imagem e o valor de $m$ ao número de bandas (ou canais).

Por exemplo, uma imagem colorida representa uma imagem com 3 bandas de cor $(m=3)$, a qual pode estar em $2 \mathrm{D}$ quando $\mathcal{I} \subset \mathbb{Z}^{2}$ ou $3 \mathrm{D}$ quando $\mathcal{I} \subset \mathbb{Z}^{3}$, sendo que cada spel é chamado de pixel (picture element) para imagens $2 \mathrm{D}$ e de voxel (volume element) para imagens 3D. Assim, cada spel $t \in \mathcal{I}$ será associado a um vetor de 3 dimensões $\vec{I}(t)=\left(I_{1}(t), I_{2}(t), I_{3}(t)\right) \in \mathbb{Z}^{3}$ que representa os valores de luz refletida em $t \in \mathcal{I}$ nos comprimentos de onda do vermelho (R, red), verde (G, green) e azul (B, blue), respectivamente.

Note que cada spel tem uma coordenada espacial em $\mathcal{I}$ e uma coordenada paramétrica $\left(I_{1}(t), I_{2}(t), \ldots, I_{m}(t)\right) \in \mathbb{Z}^{m}$, que define um espaço de parâmetros (características ou atributos) da imagem $\hat{I}$. No caso do exemplo da imagem colorida, temos um espaço de cores RGB, no qual $\vec{I}(t)$ indica uma única cor no pixel $t$. Na Figura 2.1 podemos observar o mapeamento entre coorde- 
nadas espaciais e paramétricas para uma dada imagem colorida, em que spels de mesma cor caem na mesma célula (bin) no espaço paramétrico.

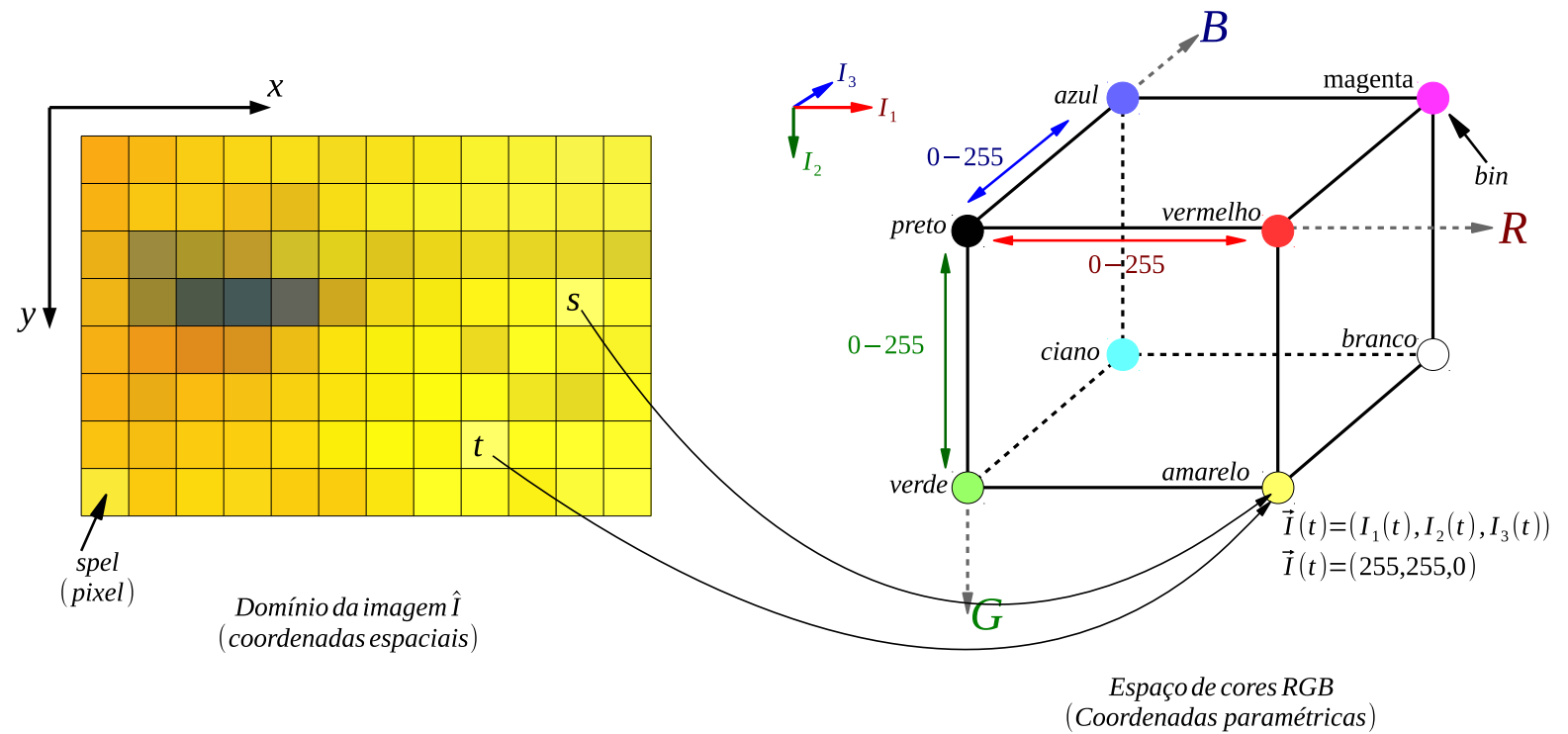

Figura 2.1: Mapeamento $\hat{I}: \mathcal{I} \rightarrow \mathbb{Z}^{m}$ a partir do dominio $\mathcal{I}$ da imagem sobre o espaço de cores $R G B$, para uma imagem colorida.

Uma imagem $\hat{I}$ em tons de cinza e bidimensional (por exemplo, uma foto em branco e preto, uma imagem de ultrasom ou uma fatia tomográfica) possui apenas uma banda $I$ (isto é, $I(t)=I_{i}(t)$ para $i=m=1$ ). Assim, uma imagem $\hat{I}$ em tons de cinza pode ser simplificada para $\hat{I}=\langle\mathcal{I}$, I , tal que para valores altos de $I(t)$ os pixels da imagem são apresentados na tela como pixels claros e para valores baixos como pixels escuros. No caso de $I(t) \in\{0,1\}$ para todo $t \in \mathcal{I}, \hat{I}$ é dita binária.

Uma imagem em tons de cinza e multidimensional define um domínio de amostragem $\mathcal{I} \subset \mathbb{Z}^{n}$, para $n>2$. Por exemplo, uma sequência espacial de fatias tomográficas é uma imagem tridimensional $\left(\mathcal{I} \subset \mathbb{Z}^{3}\right)$ e uma sequência espacial e temporal de fatias tomográficas é uma imagem tetra-dimensional $\left(\mathcal{I} \subset \mathbb{Z}^{4}\right)$.

Uma imagem multibanda é dada quando $\vec{I}$ associa a cada spel $t$ mais de um valor escalar $I_{i}(t)$, para $i=1,2, \ldots, m$ com $m>1$. Por exemplo, uma imagem de satélite, com sensor Thematic Mapper, tem $n=2$ e $m=7$, possui bandas que correspondem a imagens em tons de cinza obtidas nos comprimentos de onda do azul, vermelho, verde, infravermelho, infravermelho próximo, termal e ultravioleta.

\subsubsection{Representação de uma imagem}

A representação de uma imagem pode ser matricial ou vetorial. No caso da representação matricial a imagem é considerada como uma matriz com $n$ dimensões (por exemplo $n=3$ ), na qual cada célula terá $m$ valores $\left(I_{1}(t), I_{2}(t), \ldots, I_{m}(t)\right)$, mas a forma mais comum de armazenar os valores dos pixels é vetorialmente, fatia por fatia (ao longo do eixo $Z$ ), da esquerda para direita (ao longo do eixo $X$ ), e de cima para baixo (ao longo do eixo $Y$ ) no caso de uma imagem 3D.

Por exemplo, no caso de uma imagem em tons de cinza e bidimensional, a imagem pode ser representada na forma de uma matriz de tamanho $N \times M$ pixels ( $N$ linhas e $M$ colunas), ou como 
um vetor (Figura 2.2) tal que o índice $p$ do vetor se relaciona com as coordenadas espaciais $(x, y)$ por

$$
\begin{aligned}
p & =x+M * y \\
x & =p \% M \\
y & =p \div M
\end{aligned}
$$

tal que $\%$ e $\div$ denotam os operadores de resto e de divisão inteira, respectivamente.

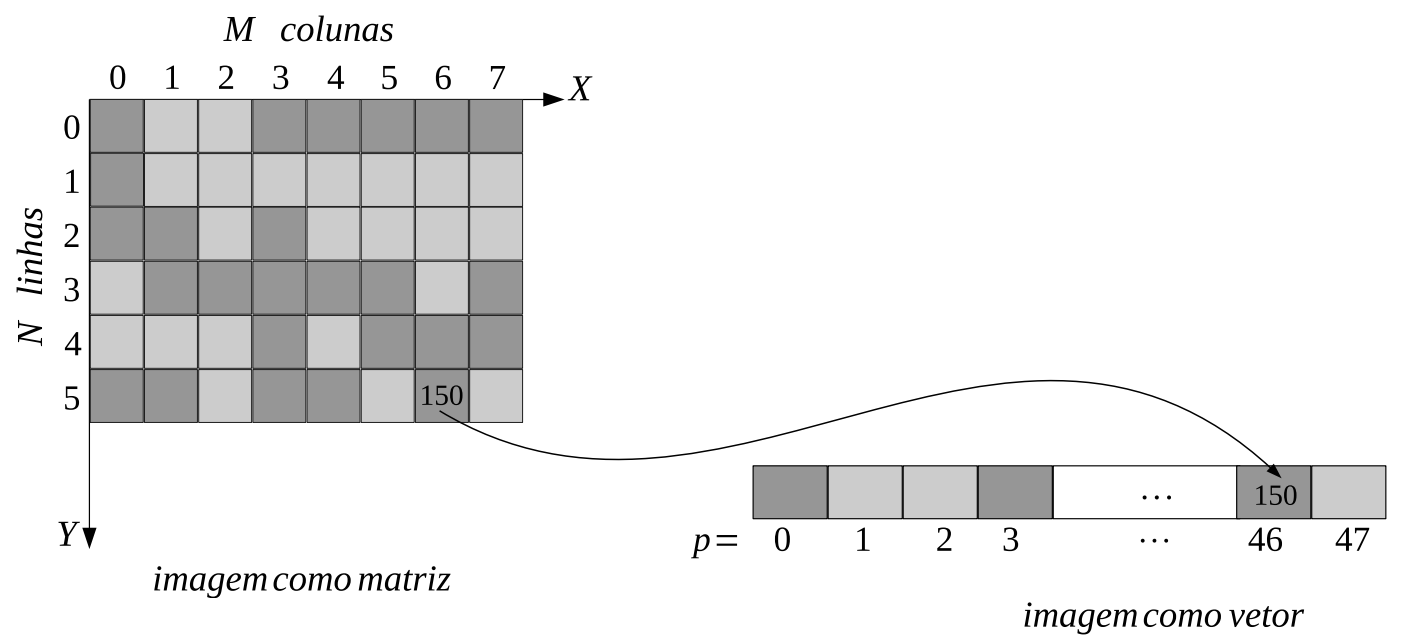

Figura 2.2: Representação de uma imagem $2 D$ em tons de cinza de forma matricial e vetorial. Para o pixel na posição $(x, y)=(6,5)$ temos que seu indice correspondente no vetor é $p=46$, com uma intensidade $I(p)=150$.

\subsection{Noções de grafos}

\subsubsection{Grafo}

Um grafo é um par $\langle\mathcal{V}, \boldsymbol{\xi}\rangle$, no qual $\mathcal{V}$ é um conjunto finito não vazio de vértices (ou nós) e $\boldsymbol{\xi}$ é um conjunto composto por pares de elementos distintos de $\mathcal{V}$, denominados arestas. Usamos $|\boldsymbol{\xi}|$ e $|\mathcal{V}|$ para denotar o número de arestas e vértices do grafo, respectivamente (Figura 2.3).

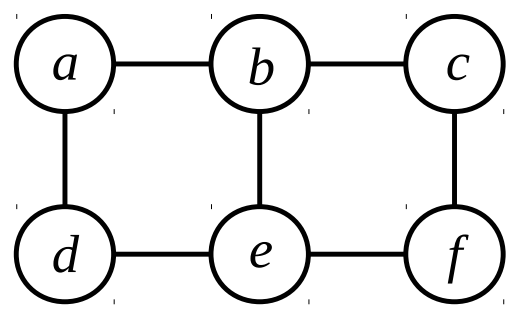

Figura 2.3: Exemplo de representação de um grafo com $\mathcal{V}=\{a, b, c, d, e, f\} \quad e$ $\boldsymbol{\xi}=\{\langle a, b\rangle,\langle b, c\rangle,\langle a, d\rangle,\langle b, e\rangle,\langle c, f\rangle,\langle d, e\rangle,\langle e, f\rangle\}$, no qual $|\mathcal{V}|=6$ e $|\boldsymbol{\xi}|=7$. 


\subsubsection{Digrafo}

Um digrafo (grafo dirigido ou orientado) é um par $\langle\mathcal{V}, \mathcal{A}\rangle$, no qual $\mathcal{V}$ é um conjunto finito não vazio de vértices e $\mathcal{A}$ é um conjunto composto por pares ordenados de elementos distintos de $\mathcal{V}$ (isto é, para $u, v \in \mathcal{V}$, os pares $\langle u, v\rangle$ e $\langle v, u\rangle$ são considerados distintos), chamados de arcos ou arestas dirigidas. Usamos a notação $\langle u, v\rangle$, em um digrafo, para indicar um arco que aponta a partir do vértice $u$ até o vértice $v$ (Figura 2.4).

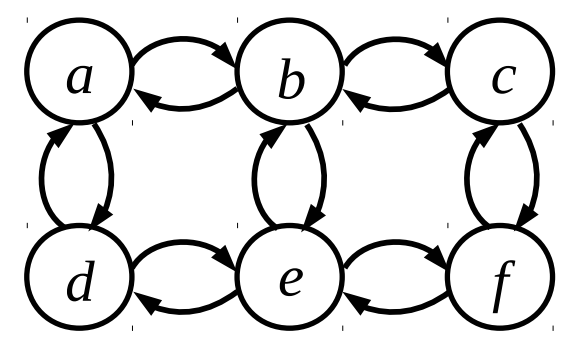

Figura 2.4: Exemplo de representação de um digrafo com $\mathcal{V}=\{a, b, c, d, e, f\} \quad e$ $\mathcal{A}=\{\langle a, b\rangle,\langle b, a\rangle,\langle b, c\rangle,\langle c, b\rangle,\langle a, d\rangle,\langle d, a\rangle,\langle b, e\rangle,\langle e, b\rangle,\langle c, f\rangle,\langle f, c\rangle,\langle d, e\rangle,\langle e, d\rangle,\langle e, f\rangle,\langle f, e\rangle\}$, no qual $|\mathcal{V}|=6$ e $|\mathcal{A}|=14$.

Dois arcos são antiparalelos se eles interligam um mesmo par de vértices com orientações opostas, isto é, para $u, v \in \mathcal{V}$ existe um arco $\langle u, v\rangle$ e outro $\langle v, u\rangle$ (Figura 2.5).

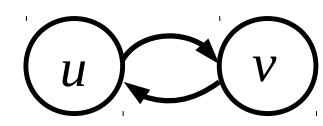

Figura 2.5: Exemplo de representação de dois arcos antiparalelos.

\subsubsection{Digrafo ponderado}

Um digrafo ponderado $G=\langle\mathcal{V}, \mathcal{A}, \omega\rangle$ é um digrafo que possui valores (pesos) associados a seus arcos e/ou vértices, em que $\omega: \mathcal{V} \cup \mathcal{A} \rightarrow \mathbb{R}$ é uma função que associa pesos aos elementos do digrafo. Usamos $\omega_{a}: \mathcal{A} \rightarrow \mathbb{R}$ para denotar os pesos associados aos arcos e $\omega_{v}: \mathcal{V} \rightarrow \mathbb{R}$ para indicar os pesos associados aos vértices.

\subsubsection{Digrafo simétrico}

Um digrafo $G=\langle\mathcal{V}, \mathcal{A}, \omega\rangle$ é simétrico se para todo arco $\langle u, v\rangle \in \mathcal{A}$ existe um arco antiparalelo $\langle v, u\rangle \in \mathcal{A}$. Note porém que podemos ter $\omega_{a}(\langle u, v\rangle) \neq \omega_{a}(\langle v, u\rangle)$ em um grafo simétrico.

\subsubsection{Subgrafo dirigido}

Um digrafo $G_{1}=\left\langle\mathcal{V}_{\mathbf{1}}, \mathcal{A}_{\mathbf{1}}, \omega\right\rangle$ é subgrafo de um digrafo $G=\langle\mathcal{V}, \mathcal{A}, \omega\rangle$, se para todo vértice $v \in \mathcal{V}_{\mathbf{1}}$ temos $v \in \mathcal{V}$ e para todo arco $\langle u, v\rangle \in \mathcal{A}_{\mathbf{1}}$ temos $\langle u, v\rangle \in \mathcal{A}$ (isto é, $\mathcal{V}_{\mathbf{1}} \subseteq \mathcal{V}$ e $\mathcal{A}_{\mathbf{1}} \subseteq \mathcal{A}$ ).

Dado um subconjunto $\mathcal{V}_{\mathbf{2}}$ de vértices de um digrafo $G=\langle\mathcal{V}, \mathcal{A}, \omega\rangle$, o subgrafo de $G$ induzido por $\mathcal{V}_{\mathbf{2}}$, denotado por $G\left[\mathcal{V}_{\mathbf{2}}\right]$, é o subgrafo formado por $\mathcal{V}_{\mathbf{2}}$ e todos os arcos $\langle u, v\rangle \in \mathcal{A}$ tal que $u$ e $v$ pertencem a $\mathcal{V}_{\mathbf{2}}$. Isto é, $G\left[\mathcal{V}_{\mathbf{2}}\right]$ é o subgrafo $G_{2}=\left\langle\mathcal{V}_{\mathbf{2}}, \mathcal{A}_{\mathbf{2}}, \omega\right\rangle$, com $\mathcal{A}_{\mathbf{2}}=\left\{\langle u, v\rangle \in \mathcal{A} \mid u, v \in \mathcal{V}_{\mathbf{2}}\right\}$. 


\subsubsection{Digrafo ponderado transposto}

A transposta $G^{T}=\left\langle\mathcal{V}, \mathcal{A}^{T}, \omega^{T}\right\rangle$ de um digrafo $G=\langle\mathcal{V}, \mathcal{A}, \omega\rangle$, é o único digrafo ponderado no mesmo conjunto de vértices $\mathcal{V}$ com todos os arcos invertidos em relação à orientação dos $\operatorname{arcos}$ correspondentes em $G$. Isto é, para qualquer arco $\langle u, v\rangle \in \mathcal{A}^{\boldsymbol{T}}$ temos o $\operatorname{arco}\langle v, u\rangle \in \mathcal{A}$ e $\omega_{a}^{T}(\langle u, v\rangle)=\omega_{a}(\langle v, u\rangle)$.

\subsubsection{Caminho}

Dado um digrafo ponderado $G=\langle\mathcal{V}, \mathcal{A}, \omega\rangle$, uma sequência de vértices $\left\langle v_{0}, v_{1}, v_{2}, \ldots, v_{i-1}, v_{i}, \ldots, v_{k}\right\rangle$ no digrafo $G$, tal que $\left\langle v_{i-1}, v_{i}\right\rangle \in \mathcal{A}$, com $i=1,2, \ldots, k$, define um caminho $\pi$ com origem no vértice $v_{0}$ e destino no vértice $v_{k}$, formado por $k \operatorname{arcos}\left(\left\langle v_{0}, v_{1}\right\rangle,\left\langle v_{1}, v_{2}\right\rangle, \ldots,\left\langle v_{k-1}, v_{k}\right\rangle\right)$, sendo $k$ o seu comprimento.

Se todos os vértices do caminho são distintos, temos um caminho simples ou elementar. Todos os caminhos considerados neste trabalho são caminhos simples. Usamos a notação $\pi_{t}$ para indicar um caminho com destino no vértice $t$. Outras letras gregas, tal como $\tau$, também podem ser usadas para denotar diferentes caminhos. Quando desejamos indicar explicitamente a origem do caminho, a notação $\pi_{r \sim t}=\left\langle v_{0}=r, v_{1}, \ldots, v_{k}=t\right\rangle$ também pode ser usada, tal que $r$ representa o vértice origem e $t$ o vértice destino (Figura 2.6a). De um modo mais geral, nós podemos usar $\pi_{\boldsymbol{S} \sim t}=\left\langle v_{0}, v_{1}, \ldots, v_{k}=t\right\rangle$ para indicar um caminho com origem restrita ao conjunto $\boldsymbol{S}$, isto é $v_{0} \in \boldsymbol{S}$ (Figura 2.6b). Um caminho $\pi_{t}=\langle t\rangle$ composto por um único vértice é denominado caminho trivial. Usamos $\pi_{t}=\pi_{s} \cdot\langle s, t\rangle$ para indicar a extensão de um caminho $\pi_{s}$, com término em $s$, por um arco $\langle s, t\rangle \in \mathcal{A}$ (Figura 2.6c).

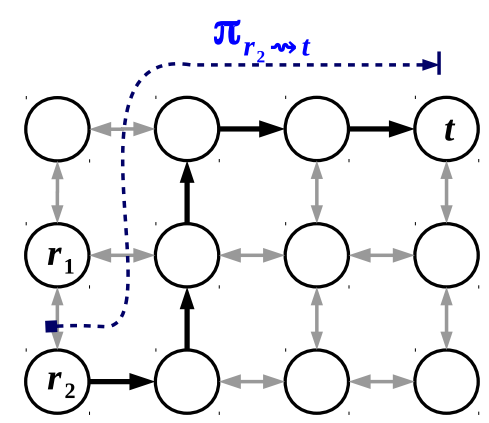

(a)

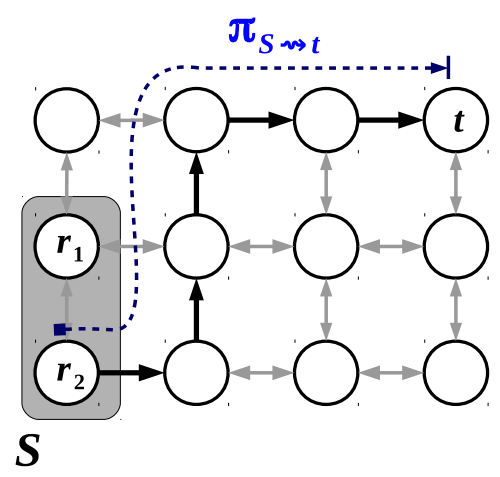

(b)

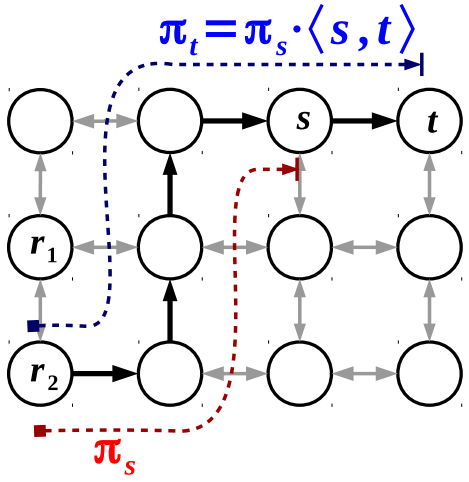

(c)

Figura 2.6: Exemplo de caminho em um digrafo de vizinhança-4 mostrando: (a) Um caminho com origem no vértice $r_{2}$ e destino no vértice $t$ (linha pontilhada azul). (b) Um caminho com origem no vértice $r_{2} \in \mathcal{S}$, tal que $\mathcal{S}=\left\{r_{1}, r_{2}\right\}$, e destino no vértice $t$. (c) Um caminho $\pi_{s}$ (linha pontilhada vermelha) estendido pelo $\operatorname{arco}\langle s, t\rangle$. 


\subsubsection{Ciclo}

Um ciclo em um grafo é um caminho $\left\langle v_{0}, v_{1}, v_{2}, \ldots, v_{k}\right\rangle$ sem vértices repetidos com exceção do primeiro e do último que coincidem (isto é, $v_{0}=v_{k}$ ), e que tem comprimento maior que 1 (isto é, $k>1$ ). Se o grafo não possui ciclos, então o grafo é denominado acíclico.

\subsubsection{Digrafo ponderado fortemente conexo}

Dado um digrafo ponderado $G=\langle\mathcal{V}, \mathcal{A}, \omega\rangle$, dois vértices $v_{i}$ e $v_{j}$ em $\mathcal{V}$ são fortemente conexos se existe um caminho de $v_{i}$ até $v_{j}$ e um caminho de $v_{j}$ até $v_{i}$. Um digrafo $G$ é fortemente conexo se todos seus vértices são fortemente conexos a qualquer outro vértice em $G$.

\subsection{Grafos a partir de imagens}

Uma relação de adjacência entre spels de uma imagem $\hat{I}=\langle\mathcal{I}, \vec{I}\rangle$ leva à definição de um digrafo ponderado, simétrico, fortemente conexo $G=\langle\mathcal{V}, \mathcal{A}, \omega\rangle$, em que os vértices em $\mathcal{V}$ são os spels em $\mathcal{I}$ e os arcos em $\mathcal{A}$ são definidos pela relação de adjacência. Em geral, $\mathcal{A}$ depende das posições relativas dos spels, o que leva a uma relação invariante à translação, mas $\mathcal{A}$ pode ser variante à translação caso dependa de outras propriedades locais da imagem, tais como cor e gradiente.

A seguir temos alguns exemplos de relações de adjacência para uma imagem 2D, nos quais os vértices do digrafo gerados correspondem aos pixels $t=(x, y)$ :

- Adjacência Circular:

$\mathcal{A}_{\mathbf{1}}=\left\{\langle s, t\rangle \in \mathcal{V}^{\mathbf{2}} \mid s \neq t \wedge d(s, t) \leqslant \rho\right\}$, tal que $d$ é a distância Euclidiana entre os pixels $s$ e $t$, e $\rho$ é uma constante dada.

- Adjacência baseada em conjunto:

$$
\begin{aligned}
& \mathcal{A}_{\mathbf{2}}=\left\{\langle s, t\rangle \in \mathcal{V}^{2} \mid t-s \in\{(-1,-1),(-1,1),(1,-1),(1,1)\}\right\} \\
& \mathcal{A}_{\mathbf{3}}=\left\{\langle s, t\rangle \in \mathcal{V}^{\mathbf{2}} \mid t-s \in\{(-1,-1),(1,-1)\}\right\}
\end{aligned}
$$

No primeiro exemplo, $\rho=1$ define uma vizinhança-4 em 2D (Figura 2.7a) e vizinhança-6 em 3D (Figura 2.7d), enquanto $\rho=\sqrt{2}$ define uma vizinhança-8 em 2D (Figura 2.7b). Valores maiores de $\rho$ em 2D fazem com que pixels que não compartilham primitivas geométricas sejam adjacentes (Figura 2.7c). Essas vizinhanças são chamadas de adjacências Euclidianas, as quais correspondem a uma relação simétrica e invariante a translação.

O segundo e terceiro exemplo (adjacências baseadas em conjunto) estão relacionados com a definição de elemento estruturante planar usada na morfologia matemática, o que pode permitir a definição de uma relação de adjacência assimétrica como no caso de $\mathcal{A}_{\mathbf{3}}$ (Figura 2.8).

Nas Figuras 2.9b-e podemos observar exemplos dos possíveis digrafos gerados a partir das relações de adjacência $\mathcal{A}_{\mathbf{1}}, \mathcal{A}_{\mathbf{2}}$ e $\mathcal{A}_{\mathbf{3}}$ entre os pixels de uma imagem 2D fornecida (Figura 2.9a). 


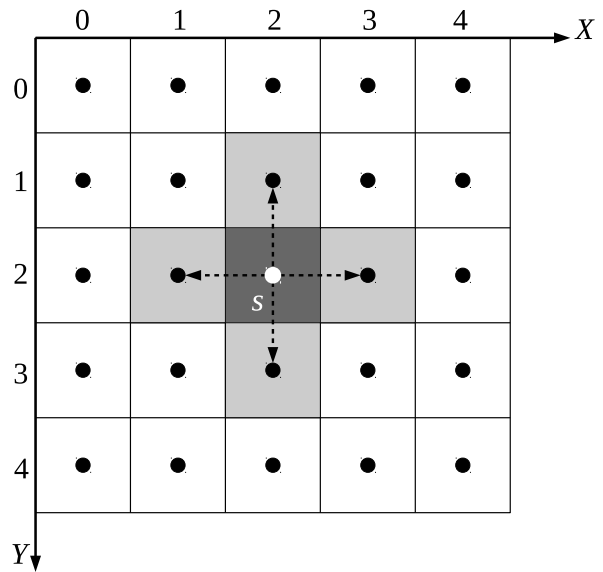

(a)

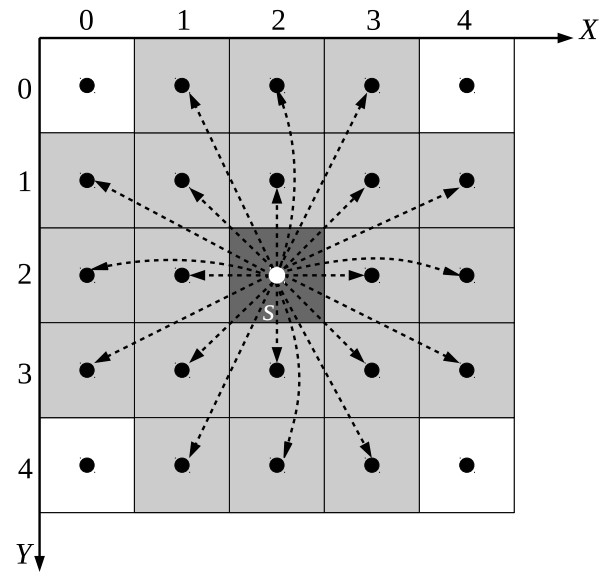

(c)

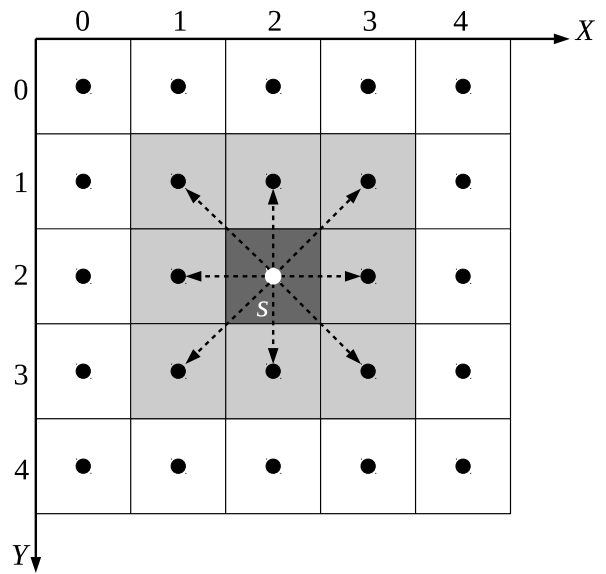

(b)

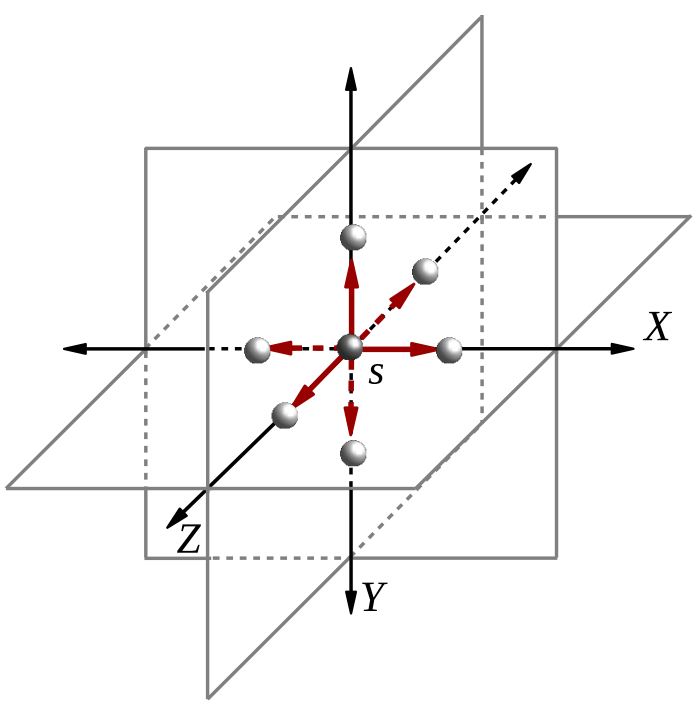

(d)

Figura 2.7: Exemplo de adjacências Euclidianas: Caso $2 D$ com raio $(a) \rho=1,(b) \rho=\sqrt{2},(c) \rho=\sqrt{5}$ e caso $3 D$ com raio (d) $\rho=1$.

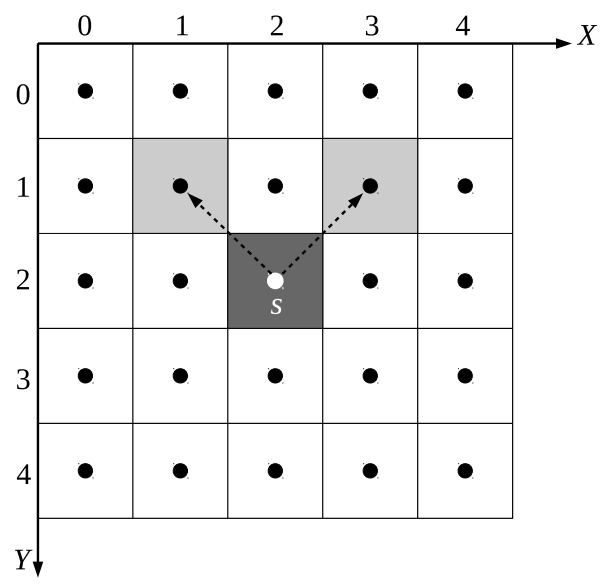

Figura 2.8: Exemplo de adjacência assimétrica, $\mathcal{A}_{\mathbf{3}}=\left\{\langle s, t\rangle \in \mathcal{V}^{2} \mid t-s \in\{(-1,-1),(1,-1)\}\right\}$ 


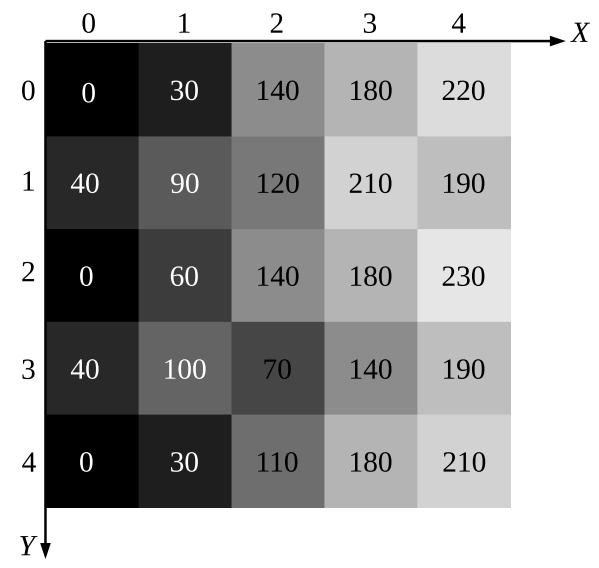

(a) $\hat{I}=\langle\mathcal{I}, I\rangle$

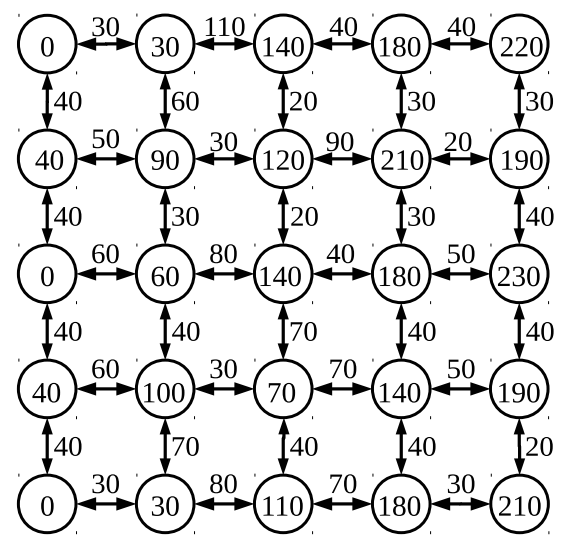

(b) $G_{1}=\left\langle\mathcal{I}, \mathcal{A}_{\mathbf{1}}, \omega\right\rangle(\rho=1)$

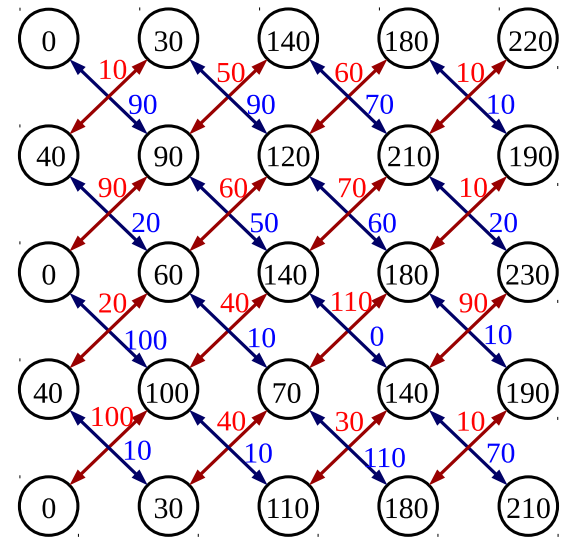

(d) $G_{3}=\left\langle\mathcal{I}, \mathcal{A}_{2}, \omega\right\rangle$

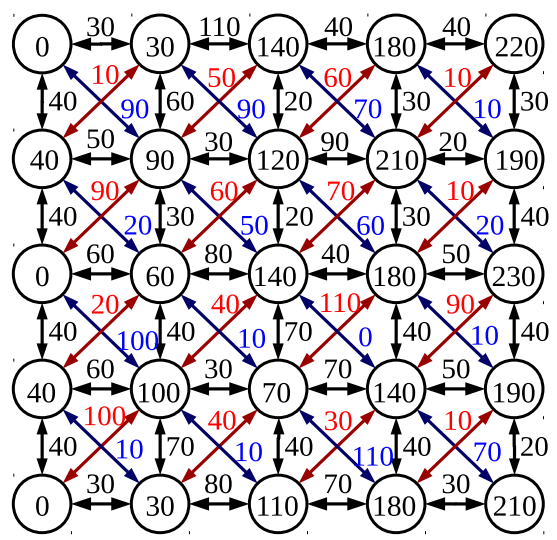

(c) $G_{2}=\left\langle\mathcal{I}, \mathcal{A}_{\mathbf{1}}, \omega\right\rangle(\rho=\sqrt{2})$

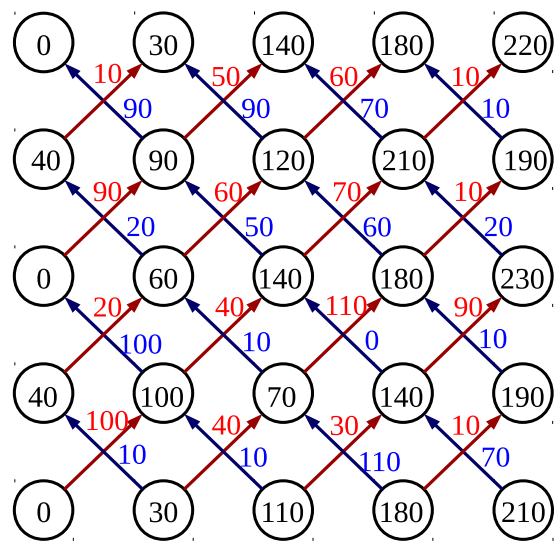

(e) $G_{4}=\left\langle\mathcal{I}, \mathcal{A}_{\mathbf{3}}, \omega\right\rangle$

Figura 2.9: Exemplo de digrafos gerados a partir de (a) uma imagem $2 D$ em tons de cinza. Os números dentro dos pixels representam os valores de intensidade (brilho) $I(t)$ para todo pixel $t \in \mathcal{I}$. Em (b) $e(c)$ temos os digrafos $G_{1}$ e $G_{2}$ gerados usando a relação de adjacência circular (ou Euclidiana) $\mathcal{A}_{1}$ com $\rho=1$ e $\rho=\sqrt{2}$, respectivamente. Em (d) e (e) temos os digrafos $G_{3}$ e $G_{4}$ gerados usando as relações de adjacência baseadas em conjunto $\mathcal{A}_{\mathbf{2}}$ e $\mathcal{A}_{\mathbf{3}}$, respectivamente. Os números dentro dos vértices representam o valor associado aos vértices do digrafo (isto é, $\omega_{v}(t)=I(t)$ para todo vértice $t$ no digrafo). As setas duplamente direcionadas representam arcos antiparalelos igualmente ponderados. Os números ao lado das setas são os valores associados aos arcos do digrafo (isto é, $\omega_{a}(\langle s, t\rangle)=|I(t)-I(s)|$ para todo arco $\langle s, t\rangle$ no digrafo). Note que somente os digrafos $G_{1}$ e $G_{2}$ são simétricos e fortemente conexos, o digrafo $G_{3}$ é simétrico e o digrafo $G_{4}$ é assimétrico. 


\subsection{Segmentação de objetos no digrafo da imagem}

Uma imagem $\hat{I}=\langle\mathcal{I}, \vec{I}\rangle$ pode conter vários objetos de interesse para uma dada aplicação. A segmentação é uma operação de processamento de imagens que reconhece e delineia esses objetos em $\hat{I}$, gerando uma imagem de rótulos como resultado da segmentação.

Uma imagem de rótulos é uma imagem em tons de cinza $\hat{L}=\langle\mathcal{I}, L\rangle$, na qual $L: \mathcal{I} \rightarrow \mathbb{Z}$ é uma função que associa todo pixel $t \in \mathcal{I}$ a um valor inteiro $L(t)$, denominado rótulo, o qual representa um identificador único para cada objeto, incluindo o fundo.

\subsubsection{Segmentação binária}

A segmentação de uma imagem $\hat{I}$ que contém um único objeto, o qual pode ser composto de vários componentes, gera como resultado uma imagem rotulada binária $\hat{L}=\langle\mathcal{I}, L\rangle$ tal que, para todo $t \in \mathcal{I}, L(t) \in\{0,1\}$, conhecida como segmentação binária, na qual 0 e 1 representam os rótulos do fundo e do objeto, respectivamente (Figura 2.10c).

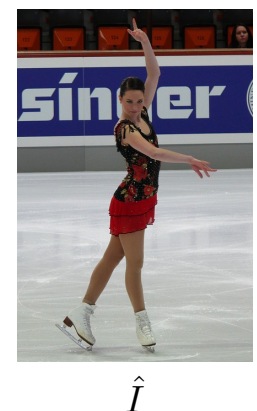

(a)

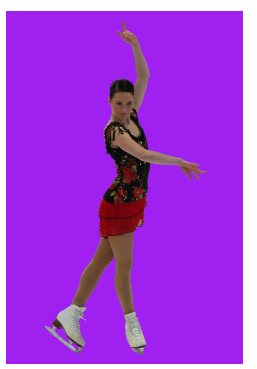

(b)

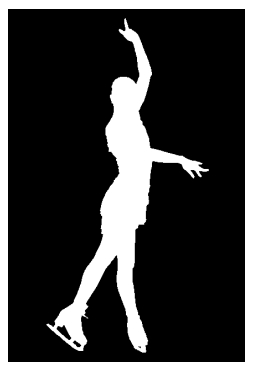

$\hat{L}=\langle\mathcal{I}, L\rangle$

(c)

Figura 2.10: Exemplo de segmentação binária: (a) Imagem $\hat{I}=\langle\mathcal{I}, \vec{I}\rangle$. (b) Imagem segmentada representando o objeto $\mathcal{O}$. (c) Imagem de rótulos $\hat{L}$. Note que o mapa de rótulos $L$ contém valores de 1 (branco em $\hat{L})$ representando o objeto e 0 (preto em $\hat{L}$ ) representando o fundo.

A seguir definiremos conceitos relacionados à segmentação binária. No entanto, esses conceitos podem ser estendidos para a segmentação com múltiplos objetos.

Definição 1 (Objeto) Um objeto $\mathcal{O}$ na imagem $\hat{I}$ é um subconjunto de $\mathcal{I}$, formado por um ou mais componentes conexos, definido por:

$$
\mathcal{O}=\{t \in \mathcal{I} \mid L(t)=1\}
$$

Definição 2 (Borda) A borda do objeto é um conjunto de vértices $s \in \mathcal{O}$ que possui ao menos um vértice vizinho adjacente $t$ pertencente ao fundo (isto é, $t \in \mathcal{I} \backslash \mathcal{O}$ ), definido por:

$$
\mathcal{B}(\mathcal{O})=\{s \in \mathcal{O} \mid \exists\langle s, t\rangle \in \mathcal{A} \wedge t \notin \mathcal{O}\}
$$

Definição 3 (Corte) Um corte no digrafo $G$ ou borda de corte do objeto $\mathcal{O}$ em $G$ é o conjunto de todos os arcos $\langle s, t\rangle \in \mathcal{A}$ que conectam os vértices representando o objeto com os vértices representando o fundo, definido por:

$$
\mathcal{C}(\mathcal{O})=\{\langle s, t\rangle \in \mathcal{A} \mid s \in \mathcal{O} \wedge t \notin \mathcal{O}\}
$$




\subsubsection{Segmentação com sementes}

Considere uma imagem $\hat{I}$ contendo um único objeto alvo $\mathcal{O}$ a ser segmentado. Um algoritmo de segmentação pode levar a múltiplos resultados, uma vez que uma imagem pode conter diferentes objetos possíveis a serem segmentados, sendo necessário algum tipo de informação que permita a identificação do objeto alvo. Na segmentação interativa, essa informação é obtida através dos marcadores na imagem fornecidos pelo usuário. Esses marcadores são conjuntos de pixels chamados de pixels sementes ou sementes. Assim, um conjunto de sementes é um conjunto de pixels identificando um objeto na imagem (incluindo o fundo).

Na segmentação binária, consideramos dois conjuntos de sementes, um conjunto $\mathcal{S}_{1}$ de sementes internas composto por pixels selecionados dentro do objeto (isto é, $\mathcal{S}_{\boldsymbol{1}} \subset \boldsymbol{O} \subset \boldsymbol{I}$ ) e um conjunto $\mathcal{S}_{\mathbf{0}}$ de sementes externas composto por pixels selecionados no fundo (isto é, $\mathcal{S}_{\mathbf{0}} \subset \mathcal{I} \backslash \mathcal{O}$ ), tal que $\mathcal{S}_{1} \cap \mathcal{S}_{0}=\varnothing$.

Seja $\mathcal{U}$ o conjunto de todos os objetos possíveis de serem segmentados na imagem $\hat{I}$. Cada objeto $\mathcal{O} \in \mathcal{U}$ é identificado com a função característica $L$ do objeto $\mathcal{O}$ definida por:

$$
L(t)= \begin{cases}1, & \text { se } t \in \mathcal{O} \\ 0, & \text { caso contrário. }\end{cases}
$$

Assim, os conjuntos de sementes $\mathcal{S}_{1}$ e $\mathcal{S}_{\mathbf{0}}$ restringem os possíveis resultados da segmentação para o subconjunto $\mathcal{U}\left(\mathcal{S}_{1}, \mathcal{S}_{\mathbf{0}}\right)$ (Equação 2.6), tal que para qualquer objeto $\mathcal{O} \in \mathcal{U}\left(\mathcal{S}_{1}, \mathcal{S}_{\mathbf{0}}\right)$, sempre se cumpre que para todo $t \in \mathcal{S}_{1}$ temos $t \in \mathcal{O}(L(t)=1)$ e para todo $t \in \mathcal{S}_{\mathbf{0}}$ temos $t \notin \mathcal{O}(L(t)=0)$.

$$
\mathcal{U}\left(\mathcal{S}_{1}, \mathcal{S}_{0}\right)=\left\{\mathcal{O} \subseteq \mathcal{V} \mid \mathcal{S}_{1} \subseteq \mathcal{O} \subseteq \mathcal{V} \backslash \mathcal{S}_{0}\right\}
$$

Por exemplo, na Figura 2.11 podemos observar que os conjuntos de sementes $\mathcal{S}_{1}$ e $\mathcal{S}_{\mathbf{0}}$ reduzem o número de soluções possíveis da segmentação para a imagem $\hat{I}$, eliminando aquelas soluções que não contêm os conjuntos de sementes $\mathcal{S}_{1}$ e $\mathcal{S}_{\mathbf{0}}$ no objeto e no fundo, respectivamente.

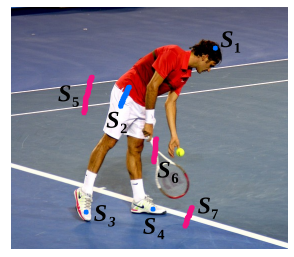

(a) $\hat{I}=\langle\mathcal{I}, \vec{I}\rangle$

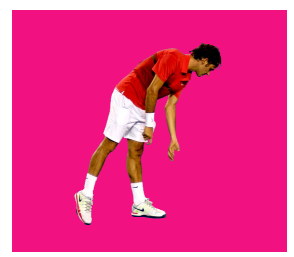

(b)

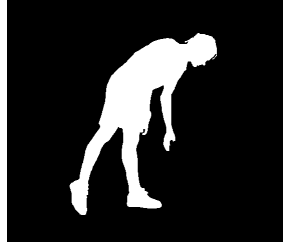

(c) $\hat{L}_{1}=\left\langle\mathcal{I}, L_{1}\right\rangle$

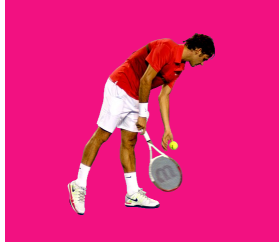

(d)

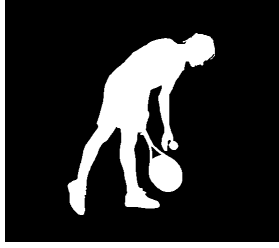

(e) $\hat{L}_{2}=\left\langle\mathcal{I}, L_{2}\right\rangle$

Figura 2.11: Exemplo de segmentação satisfazendo as restrições de sementes: (a) Imagem de entrada $\hat{I}$ com conjuntos de sementes $\mathcal{S}_{\mathbf{1}}$ e $\mathcal{S}_{\mathbf{0}}$ selecionados pelo usuário, tal que $\boldsymbol{S}_{\mathbf{1}}=\boldsymbol{S}_{\mathbf{1}} \cup \boldsymbol{S}_{\mathbf{2}} \cup \boldsymbol{S}_{\mathbf{3}} \cup \boldsymbol{S}_{\mathbf{4}}$ e $\boldsymbol{S}_{\mathbf{0}}=\boldsymbol{S}_{\mathbf{5}} \cup \boldsymbol{S}_{\mathbf{6}} \cup \boldsymbol{S}_{\mathbf{7}}$. (b) Imagem representando o resultado $\mathcal{O}_{1}$ de uma possivel segmentação da imagem $\hat{I}$, que satisfaz as restrições de sementes $\mathcal{S}_{1}$ e $\mathcal{S}_{0}\left(\mathcal{O}_{1} \in \mathcal{U}\left(\mathcal{S}_{1}, \mathcal{S}_{0}\right)\right)$. Note que esse resultado possui a função característica $L_{1}$, mostrada em (c). (d) Imagem representando o resultado $\mathcal{O}_{2}$ de uma segmentação de $\hat{I}$ que não satisfaz as restrições de sementes $\mathcal{S}_{1}$ e $\mathcal{S}_{0}\left(\mathcal{O}_{2} \notin \mathcal{U}\left(\mathcal{S}_{1}, \mathcal{S}_{0}\right)\right)$, e sua função característica correspondente $L_{2}$ em $(e)$. 


\subsection{Avaliação do desempenho dos métodos de segmentação}

O desempenho de um método de segmentação pode ser avaliado usando uma medida de acurácia, uma medida de erro de borda ou seu tempo de execução. As duas primeiras medidas avaliam a qualidade dos resultados, enquanto que a última avalia a velocidade com que os resultados são obtidos pelo método.

\subsubsection{Acurácia}

Em segmentação de imagens, a acurácia é o grau de similaridade entre a imagem de rótulos obtida da segmentação pelo método avaliado e a imagem de rótulos esperada (gabarito ${ }^{1}$ ).

Usualmente, as medidas de acurácia variam no intervalo [0,1], em que 0 representa a pior acurácia, isto é, quando as imagens de rótulos comparadas são incompatíveis, e 1 representa a melhor acurácia, isto é, quando as imagens de rótulos comparadas são idênticas.

$\mathrm{Na}$ literatura, diferentes medidas de acurácia foram propostas, uma vez que não existe uma medida universal que seja melhor para todos os casos de avaliação [Stehman (1997)]. Dentre essas medidas de avaliação, o Coeficiente de Dice [Dice (1945)], também conhecido como F-score ou Fmeasure [Labatut e Cherifi (2012); Rauber et al. (2013); van Rijsbergen (1979)], Coeficiente de similaridade de Sørensen [Sørensen (1948)] e Índice de acurácia média de Hellden [Hellden (1980)], é usado comumente para avaliar a acurácia dos métodos de segmentação de imagem baseados em região.

Sejam $\mathcal{O}^{\prime}$ e $\mathcal{O}^{*}$ os conjuntos de pixels rotulados como objeto na imagem de rótulos gerada pelo método avaliado e na imagem esperada, respectivamente. Em segmentação de imagens o Coeficiente de Dice está representado pela relação a seguir:

$$
C D=\frac{2\left|\mathcal{O}^{\prime} \cap \mathcal{O}^{*}\right|}{\left|\mathcal{O}^{\prime}\right|+\left|\mathcal{O}^{*}\right|}
$$

$\mathrm{Ou}$

$$
C D=\frac{2\left|\mathcal{O}^{\prime} \cap \mathcal{O}^{*}\right|}{\left|\mathcal{O}^{\prime} \cap \mathcal{O}^{*}\right|+\left|\mathcal{O}^{\prime} \cup \mathcal{O}^{*}\right|}
$$

tal que os delimitadores || representam a cardinalidade dos conjuntos usados nas expressões.

O resultado da segmentação gerado por qualquer método de segmentação baseado em região apresenta quatro conjuntos compostos por:

- Pixels rotulados corretamente como objeto, conhecidos como verdadeiros positivos(VP).

- Pixels rotulados corretamente como fundo, conhecidos como verdadeiros negativos(VN).

- Pixels rotulados incorretamente como objeto, conhecidos como falsos positivos(FP).

- Pixels rotulados incorretamente como fundo, conhecidos como falsos negativos(FN).

\footnotetext{
${ }^{1}$ O gabarito da imagem corresponde a uma imagem binária (branco no objeto e preto no fundo) geralmente obtida mediante uma segmentação manual.
} 
Os falsos positivos e negativos representam o erro da segmentação. Esses conjuntos podem ser visualizados em uma matriz de confusão (Tabela 2.1).

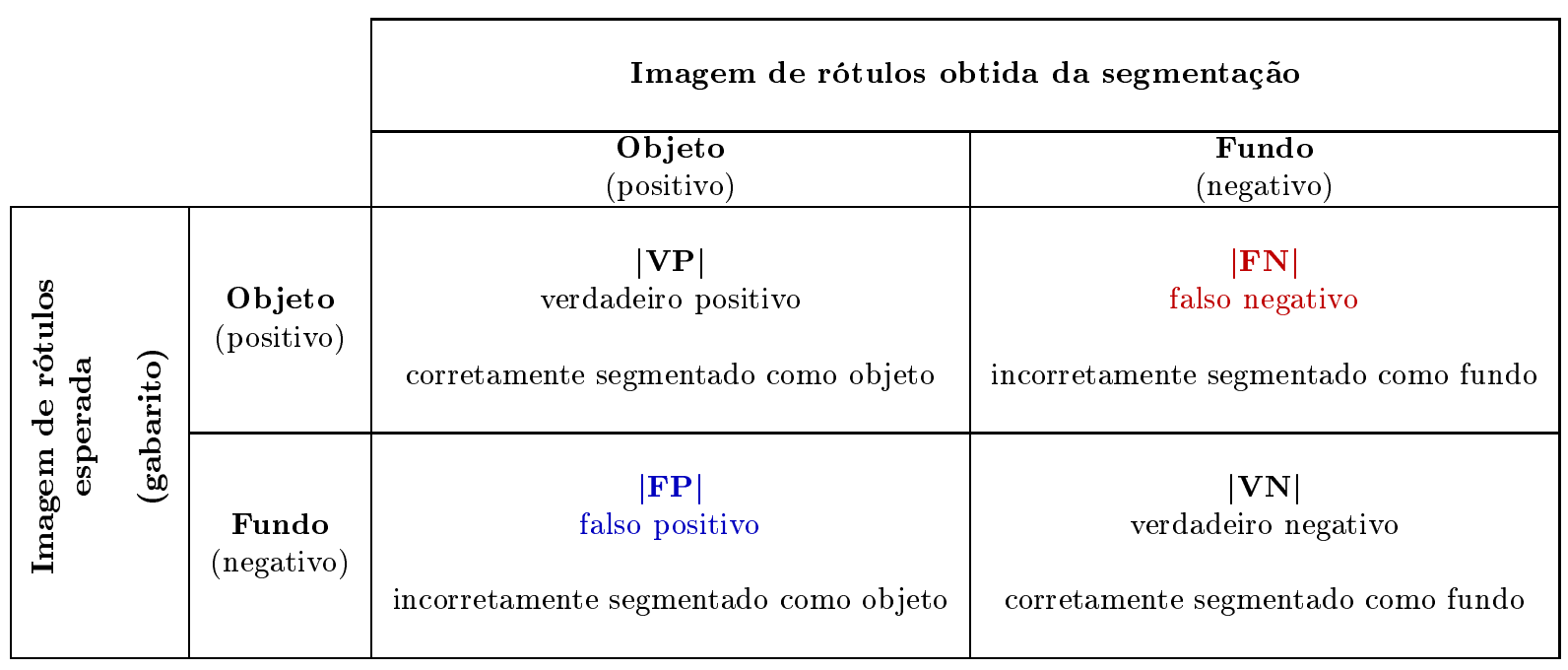

Tabela 2.1: Matriz de confusão para a segmentação binária

Em uma segmentação binária a definição do Coeficiente de Dice também pode ser expressa pela Equação 2.9 (F-measure), a qual considera a matriz de confusão (Tabela 2.1), uma vez que ela tem uma equivalência no diagrama de conjuntos usado na definição original do Coeficiente de Dice (Figura 2.12).

$$
C D=\frac{2|V P|}{2|V P|+|F N|+|F P|}
$$

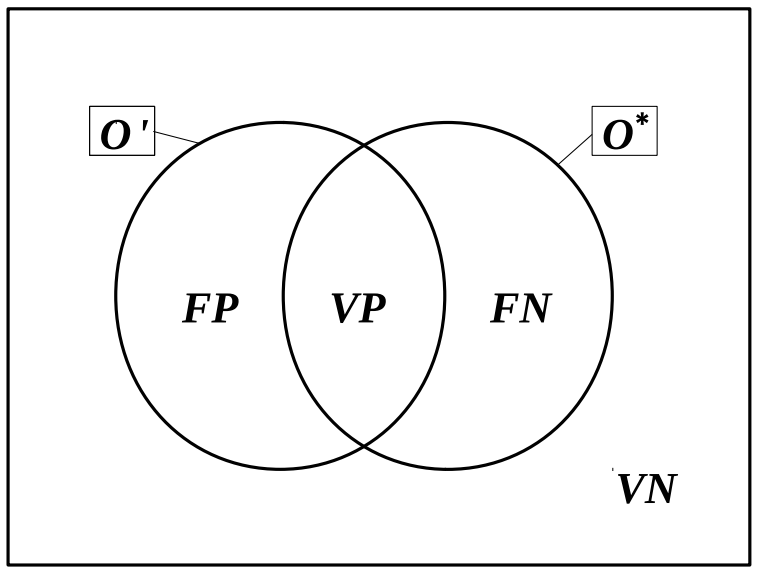

Figura 2.12: Diagrama da relação entre os conjuntos usados no Coeficiente de Dice, em que $\mathcal{O}^{\prime}$ representa o conjunto de pixels rotulados como objeto pelo método avaliado, $\mathcal{O}^{*}$ representa o conjunto de pixels rotulados como objeto no gabarito, VP (verdadeiros positivos) representa o conjunto de pixels do objeto corretamente rotulados, FP (falsos positivos) representa o conjunto de pixels do fundo incorretamente rotulados, FN (falsos negativos) representa o conjunto de pixels do objeto incorretamente rotulados e VN (verdadeiros negativos) representa o conjunto de pixels do fundo corretamente rotulados. 
Por exemplo, o valor do Coeficiente de Dice (Equação 2.7) calculado para o resultado da segmentação da imagem da Figura 2.13a, obtido via um método específico $M_{1}$, com gabarito conhecido (Figura 2.13b), é dado por:

$$
\begin{aligned}
C D_{M_{1}} & =\frac{2(71)}{82+89} \\
C D_{M_{1}} & =0.83
\end{aligned}
$$

de modo que esse valor representa a medida da acurácia para o método $M_{1}$.

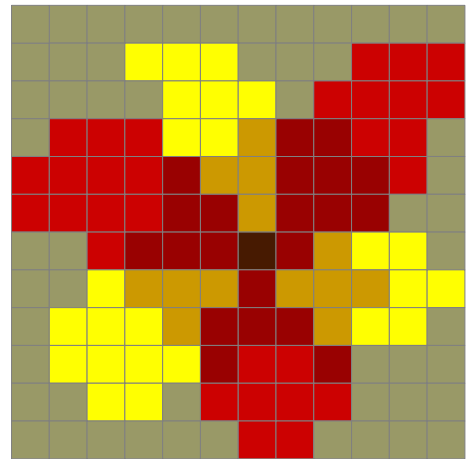

(a)

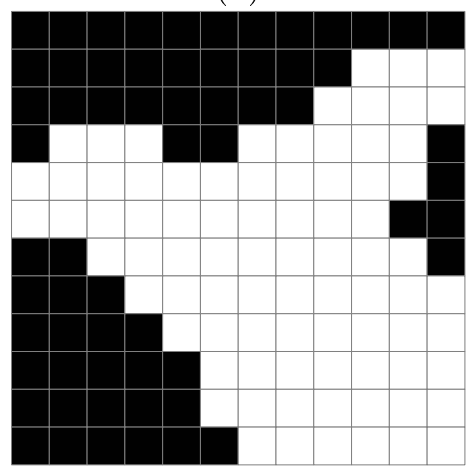

(c)

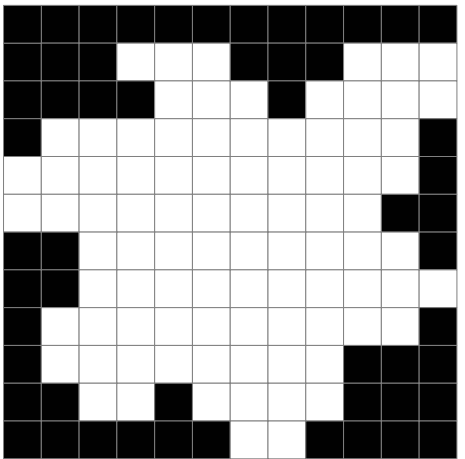

(b)

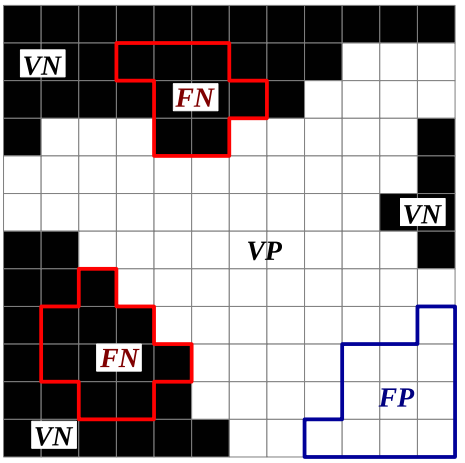

(d)

Figura 2.13: Exemplo de segmentação pelo método hipotético $M_{1}$ para uma imagem de avaliação (a), com gabarito conhecido (b), cujo resultado da segmentação (c) apresenta diferentes conjuntos que representam pixels do objeto corretamente rotulados (VP, verdadeiros positivos), pixels do fundo corretamente rotulados ( $V N$, verdadeiros negativos), pixels do fundo incorretamente rotulados ( $F P$, falsos positivos) e pixels do objeto incorretamente rotulados ( $F N$, falsos negativos).

No caso em que precisamos avaliar a acurácia de diferentes métodos de segmentação, o Coeficiente de Dice é calculado para cada um deles e, em seguida, esses resultados são comparados a fim de conhecer qual deles apresenta uma maior acurácia, isto é, um melhor desempenho na segmentação para um mesmo conjunto de imagens.

\subsubsection{Erro de borda}

O objetivo dos diferentes métodos de segmentação é encontrar soluções de alta qualidade para um determinado problema. Sempre que possível esses algoritmos deveriam identificar soluções próximas ao gabarito, com diferença mínima. Essa diferença pode ser medida com o coeficiente de Dice, como apresentado na subseção anterior. No entanto, erros em partes finas e alongadas de objetos não resultam em diferenças significativas na medida de avaliação de Dice, uma vez que a quantidade 
de pixels contidos nessas regiões finas é pequena.

Por exemplo, consideremos a imagem da Figura 2.14a, que possui um objeto com partes finas, com gabarito conhecido (Figura 2.14b), cujos resultados de segmentação pelos métodos $M_{1}$ e $M_{2}$ são apresentados nas Figuras 2.14c e 2.14f, respectivamente. Calculando os valores do coeficiente de Dice (Equação 2.7) para os resultados das segmentações pelos métodos $M_{1}$ e $M_{2}$, temos:

$$
\begin{aligned}
& C D_{M_{1}}=\frac{2(44)}{46+61}=0.82 \\
& C D_{M_{2}}=\frac{2(55)}{72+61}=0.83
\end{aligned}
$$

Note que $C D_{M_{1}}$ e $C D_{M_{2}}$ são valores muito próximos. No entanto, os resultados da segmentação possuem formas bem diferentes. Assim, uma outra medida baseada na borda do objeto segmentado em relação à borda ideal pode ser usada (Figura 2.14e,h).

Nesse trabalho nós fazemos uso de uma medida, chamada erro de borda, a qual mede a distância Euclidiana média existente entre a borda do objeto na imagem padrão (gabarito) e a borda do objeto segmentado pelo método a ser avaliado, definida a seguir.

Seja $\mathcal{B}_{4}(\mathcal{O})$ o conjunto de todos os pixels contidos na borda do objeto $\mathcal{O}$ definida por:

$$
\mathcal{B}_{4}(\mathcal{O})=\left\{s \in \mathcal{O} \mid \exists\langle s, t\rangle \in \mathcal{A}_{4} \wedge t \notin \mathcal{O}\right\}
$$

em que $\mathcal{A}_{4}$ representa uma vizinhança-4 no digrafo.

Os conjuntos $\mathcal{B}_{4}\left(\mathcal{O}^{\prime}\right)$ e $\mathcal{B}_{4}\left(\mathcal{O}^{*}\right)$ representam os conjuntos de pixels contidos na borda do objeto segmentado $\mathcal{O}^{\prime}$ e do objeto ideal $\mathcal{O}^{*}$, respectivamente.

Seja $r_{i} \in \mathcal{B}_{4}\left(\mathcal{O}^{\prime}\right)$ o pixel mais próximo ao pixel $s_{i} \in \mathcal{B}_{4}\left(\mathcal{O}^{*}\right)$ segundo a distância Euclidiana $d\left(r_{i}, s_{i}\right)$. Isto é, para qualquer $r^{\prime} \in \mathcal{B}_{4}\left(\mathcal{O}^{\prime}\right)$ temos que $d\left(r_{i}, s_{i}\right) \leq d\left(r^{\prime}, s_{i}\right)$.

A medida de erro de borda de um objeto segmentado $\mathcal{O}^{\prime}$ é dada por:

$$
\text { Erro de borda }\left(\mathcal{O}^{\prime}\right)=\frac{1}{n b} \sum_{i=1}^{n b} d\left(r_{i}, s_{i}\right)
$$

em que $n b$ é o número de pixels em $\boldsymbol{B}_{4}\left(\mathcal{O}^{*}\right), d\left(r_{i}, s_{i}\right)=\left\|r_{i}-s_{i}\right\|$ representa a distância Euclidiana entre os pixels $r_{i}$ e $s_{i}$, e $r_{i}=\operatorname{argmin} d\left(r, s_{i}\right)$.

$$
r \in \mathcal{B}_{4}\left(\mathcal{O}^{\prime}\right)
$$

Por exemplo, considere os métodos hipotéticos $M_{1}$ e $M_{2}$ da Figura 2.14. Calculando o valor do erro de borda (Equação 2.12) para o resultado da segmentação pelo método $M_{1}$ e o gabarito temos:

$$
\text { Erro de borda }\left(\mathcal{O}_{M_{1}}\right)=\frac{1}{45} \times 41.23=0.92
$$

e para o método $M_{2}$ e o gabarito temos:

$$
\text { Erro de borda }\left(\mathcal{O}_{\boldsymbol{M}_{\mathbf{2}}}\right)=\frac{1}{45} \times 6=0.13
$$

Note que os valores obtidos permitem identificar uma diferença significativa entre os métodos $M_{1}$ e $M_{2}$.

Assim, no caso de objetos com partes finas e alongadas, a medida de erro de borda permite identificar uma diferença significativa para os diferentes métodos de segmentação a serem avaliados. 


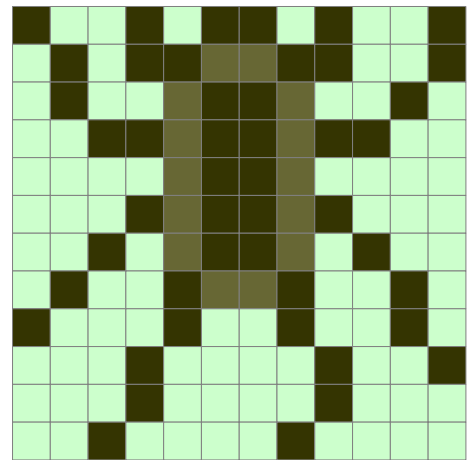

(a) $\hat{I}$

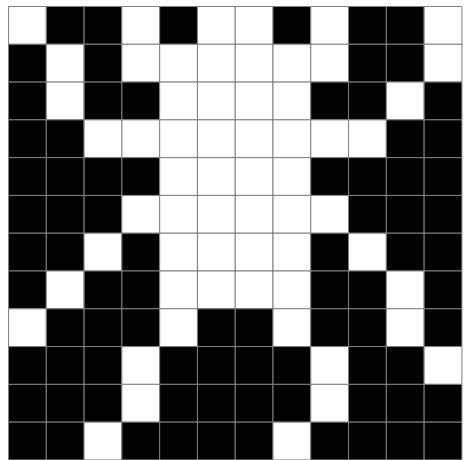

(b) $\hat{L}_{\text {ideal }}$

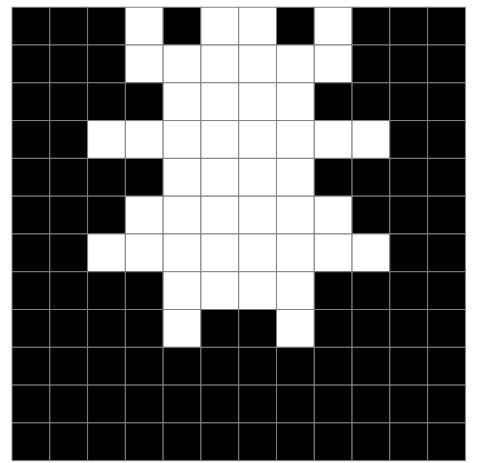

(c) $\hat{L}_{M_{1}}$

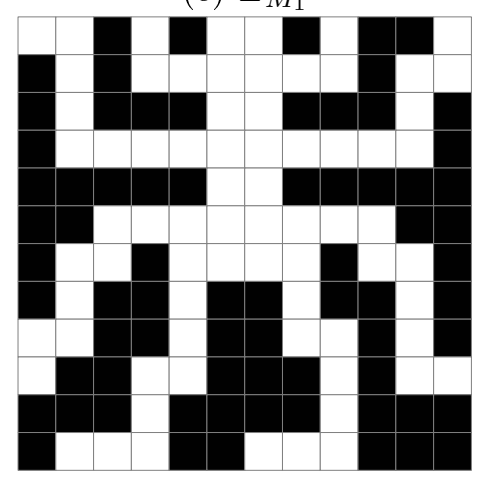

(f) $\hat{L}_{M_{2}}$

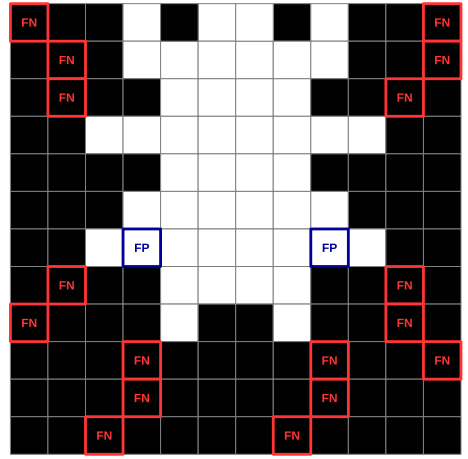

(d)

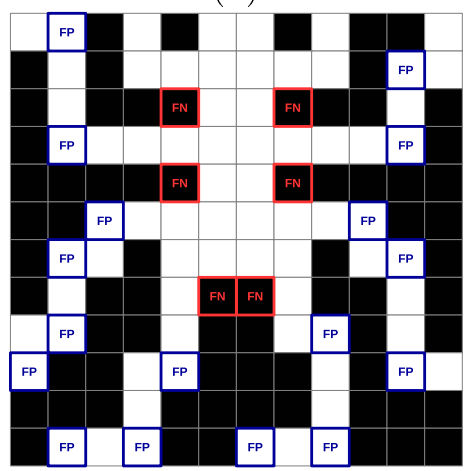

(g)

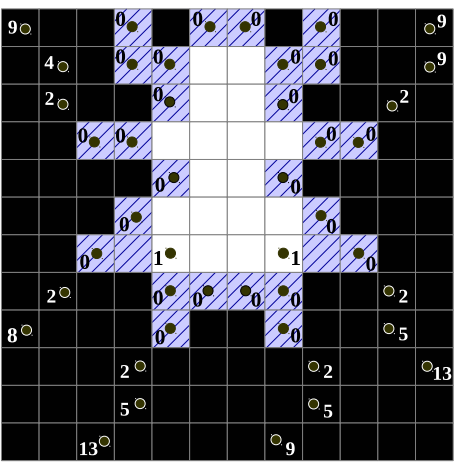

(e)

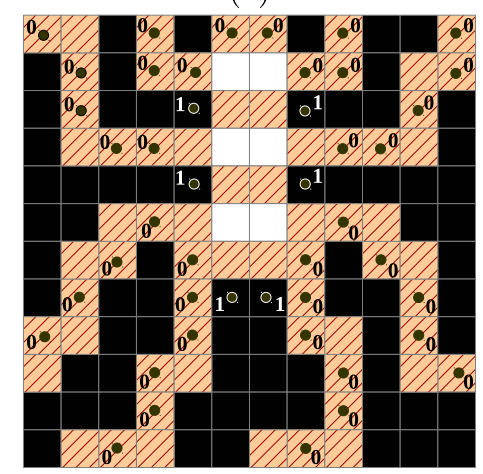

(h)

Figura 2.14: Exemplo comparativo do uso do Coeficiente de Dice e Erro de Borda considerando um digrafo de adjacência 4-conexo: (a) Imagem $\hat{I}$ de avaliação. (b) Gabarito da imagem $\hat{I}$. Em (c) e (f) são mostradas as imagens de rótulos $\hat{L}_{M_{1}}$ e $\hat{L}_{M_{2}}$, obtidas com dois métodos hipotéticos $M_{1}$ e $M_{2}$, respectivamente. Em (d) $e(g)$ são apresentados os diferentes conjuntos de pixels incorretamente rotulados como objeto ( $F P$, falsos positivos) e incorretamente rotulados como fundo (FN, falsos negativos), para os métodos $M_{1}$ e $M_{2}$. Em (e) $e$ (h) são apresentadas as bordas consideradas para obter a medida de erro de borda para os métodos $M_{1}$ e $\mathrm{M}_{2}$. Os pontos nos quadros representam a borda do objeto ideal (gabarito), tal que os números ao lado deles representam a distância Euclidiana quadrada dos mesmos até o pixel mais próximo na borda do objeto segmentado (quadros com cor azul e laranja). 


\section{Capítulo 3}

\section{Métodos relacionados}

Neste capítulo apresentamos os diferentes métodos em grafos que estão relacionados ao nosso método proposto.

\subsection{Transformada Imagem-Floresta (IFT)}

A IFT é um algoritmo usado para o desenvolvimento de operadores de processamento de imagens, com aplicações em filtragem [Falcão et al. (2001)], segmentação [Falcão e Bergo (2004); Miranda et al. (2011); Spina et al. (2012)], representação de formas [Falcão e da Cunha (2001)]), no qual uma imagem é transformada em um grafo e este em uma floresta de caminhos ótimos (custo mínimo ou força de conexidade máxima).

O conceito de um grafo derivado da imagem é usado direta ou indiretamente por várias técnicas de análise de imagens, as quais embora possuam conceitos similares são apresentadas como métodos não relacionados, levando a diferentes arcabouços, como, Watershed [Couprie et al. (2010); Cousty et al. (2010)], Random Walks [Grady (2006)], Fuzzy Connectedness [Ciesielski et al. (2007)], Graph Cuts [Boykov e Funka-Lea (2006); Vicente et al. (2008)], Distance Cut [Bai e Sapiro (2007)] e Grow Cut [Vezhnevets e Konouchine (2005)]. Dependendo do arcabouço usado, os pesos dos arcos entre os pixels do digrafo podem ser interpretados como uma medida de similaridade, função de velocidade, afinidade, custo ou distância. A IFT unifica e estende várias dessas técnicas fazendo uso da busca e otimização de conexidade em grafos, essencialmente generalizando o algoritmo de Dijkstra para funções de conexidade mais gerais.

Nesta seção apresentamos a IFT de um modo mais específico para o problema da segmentação baseada em regiões.

\subsubsection{Definição}

Para uma dada imagem $\hat{I}=\langle\mathcal{I}, I\rangle$, uma relação de adjacência em $\mathcal{I}$ define um digrafo $G=\langle\mathcal{V}, \mathcal{A}, \omega\rangle$ derivado da imagem $\hat{I}$. Assim, na IFT uma imagem $2 \mathrm{D} / 3 \mathrm{D}$ pode ser interpretada como um digrafo $G$, no qual os vértices $\mathcal{V}$ são os pixels/voxels da imagem no seu domínio $\mathcal{I} \subset \mathbb{Z}^{\boldsymbol{n}}$, os arcos são os pares de pixels ordenados $\langle s, t\rangle \in \mathcal{A} \subset \mathcal{I} \times \mathcal{I}$ e cada arco $\langle s, t\rangle \in \mathcal{A}$ tem um peso $\omega(\langle s, t\rangle)=\omega_{a}(\langle s, t\rangle) \geqslant 0$ associado, que pode ser dado por uma medida de dissimilaridade entre os pixels $s$ e $t$, bem como também cada vértice $t \in \mathcal{I}$ pode ter associado um peso $\omega(t)=\omega_{v}(t) \geqslant 0$.

Seja $\Pi(G, t)$ o conjunto de todos os caminhos possíveis no digrafo $G$ com destino no vértice 
$t, \boldsymbol{\Pi}=\bigcup_{t \in \mathcal{V}} \Pi(G, t)$ o conjunto de todos possíveis caminhos em $G$ e $\boldsymbol{F}$ um conjunto de valores de custo totalmente ordenado. A seguir apresentamos alguns conceitos usados na IFT.

Definição 4 (Função de custo) Uma função de custo $f: \Pi \rightarrow \boldsymbol{F}$ associa a qualquer caminho $\pi_{r \sim t} \in \Pi$ um valor de custo $f\left(\pi_{r \sim t}\right) \in \boldsymbol{F}$, sendo que seu inverso indica a força de conexidade entre o vértice $t$ e o vértice raiz/origem $r$ do caminho.

Geralmente, $f$ depende dos pesos $\omega_{a}$ associados aos arcos que interligam os vértices compondo o caminho, mas $f$ também pode depender dos pesos associados a esses vértices. Os pesos são normalmente baseados nas propriedades locais da imagem tais como cor, gradiente e posição do vértice ao longo do caminho, mas podem também incluir propriedades globais do objeto.

Os exemplos mais comuns de funções de custo são as funções $f_{\max }$ e $f_{\text {sum }}$, definidas pelas Equações 3.1 e 3.2 respectivamente, nas quais $\pi_{s}$ é qualquer caminho terminado em $s$ e $H(t)$ é um custo inicial fixo (handicap) para qualquer caminho trivial iniciando em $t$.

$$
\begin{aligned}
f_{\max }(\langle t\rangle) & =H(t) \\
f_{\max }\left(\pi_{s} \cdot\langle s, t\rangle\right) & =\max \left\{f_{\max }\left(\pi_{s}\right), \omega_{a}(\langle s, t\rangle)\right\} \\
f_{\text {sum }}(\langle t\rangle) & =H(t) \\
f_{\text {sum }}\left(\pi_{s} \cdot\langle s, t\rangle\right) & =f_{\text {sum }}\left(\pi_{s}\right)+\omega_{a}(\langle s, t\rangle)
\end{aligned}
$$

Em geral, o valor de $H(t)$ é dado pela Equação 3.3, na qual $\mathcal{S} \subseteq \mathcal{I}$ é um conjunto de pixels sementes (marcadores definidos pelo usuário).

$$
H(t)= \begin{cases}-1 & , \text { se } t \in \mathcal{S} \\ +\infty & , \text { caso contrário }\end{cases}
$$

Um caso particular da função $f_{\max }$ é a função $f_{\text {peak }}$ definida pela Equação 3.4 , em que $\omega_{a}(\langle s, t\rangle)=\omega_{v}(t)=I(t)$.

$$
\begin{aligned}
f_{\text {peak }}(\langle t\rangle) & =H(t) \\
f_{\text {peak }}\left(\pi_{s} \cdot\langle s, t\rangle\right) & =\max \left\{f_{\text {peak }}\left(\pi_{s}\right), \omega_{v}(t)\right\}
\end{aligned}
$$

De uma forma mais geral, as funções $f_{\max }$ e $f_{\text {sum }}$ pertencem à classe de funções monotonicamente incrementais (MI).

Definição 5 (Função monotonicamente incremental) Uma função $f_{m i}$ é monotonicamente incremental (MI) se satisfaz as expressões a seguir:

$$
\begin{aligned}
f_{m i}(\langle t\rangle) & =H(t) \\
f_{m i}\left(\pi_{s} \cdot\langle s, t\rangle\right) & =f_{m i}\left(\pi_{s}\right) \odot\langle s, t\rangle
\end{aligned}
$$

tal que $\odot: \boldsymbol{F} \times \mathcal{A} \rightarrow \boldsymbol{F}$ é uma operação binária que satisfaz as condições:

(M1) $\nu^{\prime} \geqslant \nu \Rightarrow \nu^{\prime} \odot\langle s, t\rangle \geqslant \nu \odot\langle s, t\rangle$ 
(M2) $\nu \odot\langle s, t\rangle \geqslant \nu$

para $\nu, \nu^{\prime} \in \boldsymbol{F}$ e qualquer arco $\langle s, t\rangle \in \mathcal{A}$. Note que $\odot$ depende apenas do custo de $\pi_{s}$ e não de qualquer outra propriedade de $\pi_{s}$.

Definição 6 (Caminho ótimo) Um caminho $\pi_{t}$ é ótimo se $f\left(\pi_{t}\right) \leqslant f\left(\pi_{t}^{\prime}\right)$ para qualquer outro caminho $\pi_{t}^{\prime} \in \mathbf{\Pi}(\boldsymbol{G}, \boldsymbol{t})$, independentemente da sua origem. Assim, para cada vértice $t \in \mathcal{I}$, temos um valor único $V_{o p t}^{f}(t)$ de custo de um caminho ótimo com destino em $t$, definido por:

$$
V_{o p t}^{f}(t)=\min _{\pi_{t} \in \boldsymbol{\Pi}(\boldsymbol{G}, \boldsymbol{t})}\left\{f\left(\pi_{t}\right)\right\}
$$

Definição 7 (Mapa de predecessores) Um mapa de predecessores $P$ é uma função que associa cada vértice $t \in \mathcal{I}$ a algum outro vértice adjacente $s \in \mathcal{I}$ (isto é, $P(t)=s$ ), tal que $\langle s, t\rangle \in \mathcal{A}$, ou um marcador nil $\notin \mathcal{I}$ (isto é, $P(t)=$ nil) para todo $t$ raiz do mapa.

Uma floresta de espalhamento é um mapa de predecessores $P$ que não contém ciclos (Figura 3.1b), isto é, a aplicação sucessiva da volta pelo mapa de predecessores a partir de cada vértice $t \in \mathcal{I}$ leva a um vértice raiz $R(t)$ tal que $P(R(t))=n i l$. Assim, para qualquer pixel $t \in \mathcal{I}$, a floresta $P$ define um caminho $\pi_{t}^{P}$ recursivamente como $\langle t\rangle$, se $P(t)=n i l$ (Figura $3.1 \mathrm{c}$ ), e $\pi_{s}^{P} \cdot\langle s, t\rangle$ se $P(t)=s \neq n i l$ (Figura 3.1d).

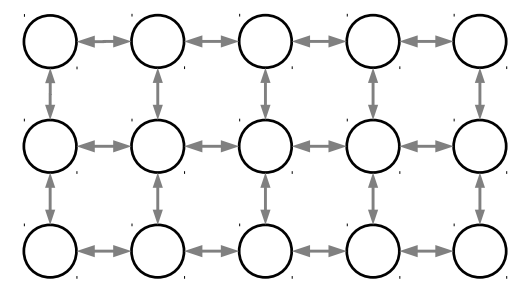

(a)

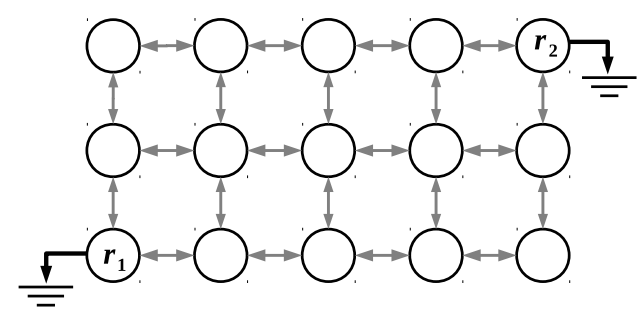

(c)

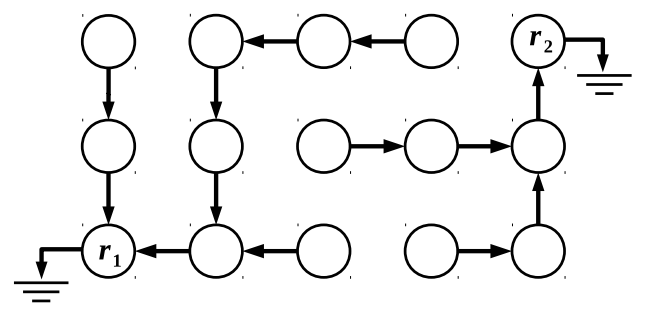

(b)

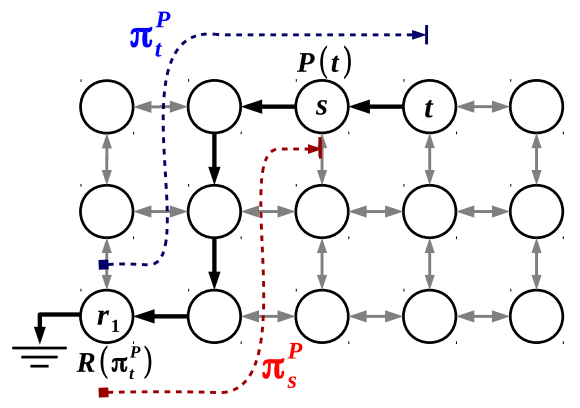

(d)

Figura 3.1: Exemplo de uma floresta de espalhamento na IFT: (a) Um digrafo $G$ de vizinhança-4. (b) Uma floresta de espalhamento $P$, obtida a partir do digrafo $G$, com dois vértices raizes $r_{1}$ e $r_{2}$. A floresta $P$ define em (c) dois caminhos triviais $\pi_{r_{1}}^{P}=\left\langle r_{1}\right\rangle$ e $\pi_{r_{2}}^{P}=\left\langle r_{2}\right\rangle$ no digrafo $G$, e em (d) um caminho $\pi_{t}^{P}=\pi_{s}^{P} \cdot\langle s, t\rangle$ (linhas pontilhadas) representado no sentido inverso, tal que $s=P(t)$ é o nó predecessor de $t$ e $r_{1}=R\left(\pi_{t}^{P}\right)$ é o pixel raiz/origem do caminho $\pi_{t}^{P}$.

Uma floresta de espalhamento $P$ é uma floresta de caminhos ótimos se para todo $t \in \mathcal{I}, \pi_{t}^{P}$ é um caminho ótimo. Note que qualquer caminho $\pi_{t}^{P}$ definido por uma floresta de caminhos ótimos $P$ é um caminho hereditariamente ótimo de acordo com a seguinte definição: 
Definição 8 (Caminho hereditariamente ótimo) Um caminho $\pi_{t_{n}}=\left\langle t_{1}, t_{2}, \ldots, t_{n}\right\rangle$ é hereditariamente ótimo se todos os caminhos $\pi_{t_{i}}=\left\langle t_{1}, t_{2}, \ldots, t_{i}\right\rangle$ para $i=1,2, \ldots, n$ são caminhos ótimos.

A IFT toma uma imagem $\hat{I}=\langle\mathcal{I}, I\rangle$, uma função de custo $f$ e uma relação de adjacência $\mathcal{A}$, transforma a imagem $\hat{I}$ num digrafo $G=\langle\mathcal{I}, \mathcal{A}, \omega\rangle$ e atribui um caminho ótimo para todo vértice $t \in \mathcal{I}$ tal que uma floresta de caminhos ótimos $P$ é obtida, isto é, uma floresta de espalhamento (mapa de predecessores) na qual todos os caminhos calculados $\pi_{t}^{P}$ são ótimos. Porém, $f$ deve satisfazer algumas condições, conforme será discutido na Seção 3.3 .

\subsubsection{Função de custo de caminho não MI}

A IFT garante resultados ótimos para uma classe de funções que contém as funções MI (Seção 3.3). No entanto, alguns outros trabalhos consideram o uso de funções não MI (FNMI), como $f_{\text {euc }}$ (Equação 3.7) com adjacência 8-conexo, para calcular uma aproximação da distância Euclidiana (EDT) [Falcão et al. (2002); Torres et al. (2004)]. Também outros trabalhos têm sugerido algumas vantagens práticas do uso das FNMI no contexto da segmentação de imagem [Herman e Carvalho (2001); Miranda et al. (2008)].

Mais recentemente, Strand et al. propuseram o Minimum Barrier Distance (MBD) [Strand et al. (2013)], o qual, numa configuração digital, leva a uma função de conexidade não MI (Equação 3.9). Em comparações empíricas de segmentação de imagem, um resultado favorável tem sido reportado para MBD [Strand et al. (2013) ${ }^{1}$.

Também, em outros trabalhos recentes, tem sido provado que algumas funções de custo de caminho não MI podem levar a resultados ótimos de acordo com outros critérios de otimalidade, tal como uma medida de corte em grafo [Mansilla e Miranda (2013a,b); Miranda e Mansilla (2014)], ou como uma função de energia para perseguição ótima de bordas [Miranda et al. (2011, 2012)]. Isso trouxe novas luzes para a pesquisa envolvendo FNMI [Mansilla et al. (2013a)]. Mais tarde, as FNMI também foram demonstradas serem úteis para a geração de superpixels [Barreto Alexandre et al. (2015)].

Por exemplo, algumas funções de custo não MI são:

$$
\begin{aligned}
f_{\text {euc }}(\langle t\rangle) & = \begin{cases}0, & \text { se } t \in \mathcal{S} \\
+\infty, & \text { caso contrário }\end{cases} \\
f_{\text {euc }}\left(\pi_{r \sim s} \cdot\langle s, t\rangle\right) & =\|t-r\|^{2}
\end{aligned}
$$

\footnotetext{
${ }^{1}$ Em [Strand et al. (2013)], foi considerado um algoritmo que encontra uma aproximação do Minimum Barrier Distance, calculando duas IFTs separadamente, uma para cada termo na Equação 3.9.
} 
tal que $\mathcal{S}$ é um conjunto de sementes, $\|t-r\|$ indica a distância Euclidiana entre os pixels $t$ e $r$. As funções $f_{\max }^{\omega_{v}}$ e $f_{\min }^{\omega_{v}}$ usadas em $f_{\downarrow}$ são funções que tomam os valores máximo e mínimo da intensidade ao longo do caminho, respectivamente, conforme definido a seguir

$$
\begin{aligned}
f_{\max }^{\omega_{v}}(\langle t\rangle) & =\omega_{v}(t) \\
f_{\max }^{\omega_{v}}\left(\pi_{s} \cdot\langle s, t\rangle\right) & =\max \left\{f_{\max }^{\omega_{v}}\left(\pi_{s}\right), \omega_{v}(t)\right\} \\
f_{\min }^{\omega_{v}}(\langle t\rangle) & =\omega_{v}(t) \\
f_{\min }^{\omega_{v}}\left(\pi_{s} \cdot\langle s, t\rangle\right) & =\min \left\{f_{\min }^{\omega_{v}}\left(\pi_{s}\right), \omega_{v}(t)\right\}
\end{aligned}
$$

tal que $\omega_{v}(t)=I(t)$ representa a intensidade do pixel $t$.

\subsection{Segmentação por competição de sementes}

Na segmentação via transformada imagem-floresta por competição de sementes (IFT-SC, IFT Segmentation by Seed Competition), são considerados dois conjuntos de sementes, um conjunto $\mathcal{S}_{1}$ de sementes internas composto por pixels selecionados dentro do objeto e um conjunto $\mathcal{S}_{\mathbf{0}}$ de sementes externas composto por pixels selecionados no fundo, tal que $\mathcal{S}_{\mathbf{1}} \cap \mathcal{S}_{\mathbf{0}}=\varnothing$. Neste paradigma, as sementes internas $\left(\mathcal{S}_{\mathbf{1}}\right)$ e externas $\left(\mathcal{S}_{\mathbf{0}}\right)$ competem por seus pixels mais fortemente conexos, de modo que a imagem é particionada em duas florestas de caminhos ótimos, uma enraizada nas sementes internas, definindo o objeto, e outra enraizada nas sementes externas, representando o fundo [Miranda e Falcão (2009)].

O resultado da segmentação é dada pela imagem de rótulos $\hat{L}$, cujos rótulos são definidos por:

$$
L(t)= \begin{cases}1, & \text { se } R\left(\pi_{t}^{P}\right) \in \mathcal{S}_{\mathbf{1}}, \\ 0, & \text { caso contrário. }\end{cases}
$$

tal que $R\left(\pi_{t}^{P}\right)$ é a raiz do caminho $\pi_{t}^{P}$ com término no pixel $t$ obtido da floresta $P$ calculada pela IFT.

Por questões de eficiência, o algoritmo da IFT (Algoritmo 1, pág. 27) já propaga os rótulos das raízes (sementes) para todos vértices do digrafo $G$, durante a sua execução.

\subsubsection{Exemplo de segmentação}

Um exemplo de segmentação baseada em região usando a IFT é apresentado na Figura 3.2. Os conjuntos de sementes $\mathcal{S}_{1}$ e $\mathcal{S}_{\mathbf{0}}$ são colocados na imagem para indicar pixels do objeto e fundo, respectivamente. A função $f_{\text {max }}$ é escolhida com $H(t)=-1$, para $t \in \mathcal{S}_{\mathbf{1}} \cup \mathcal{S}_{\mathbf{0}}$, e $H(t)=+\infty$ caso contrário. O peso $\omega_{a}(\langle s, t\rangle)$ dos arcos é calculado de forma a ser o maior possível na borda do objeto desejado em relação aos pesos que estão dentro e fora do objeto. Os conjuntos de sementes $\mathcal{S}_{1}$ e $\mathcal{S}_{\mathbf{0}}$ competem entre si e a imagem é dividida em duas florestas de caminhos ótimos, sendo o objeto definido pela floresta de caminhos ótimos com raízes no conjunto de suas sementes internas.

Na Figura 3.3 podemos observar um exemplo do processo de segmentação a partir de sementes em $\mathcal{S}$ para um objeto simples usando a mesma função $f_{\max }$ e regras de inicialização. 


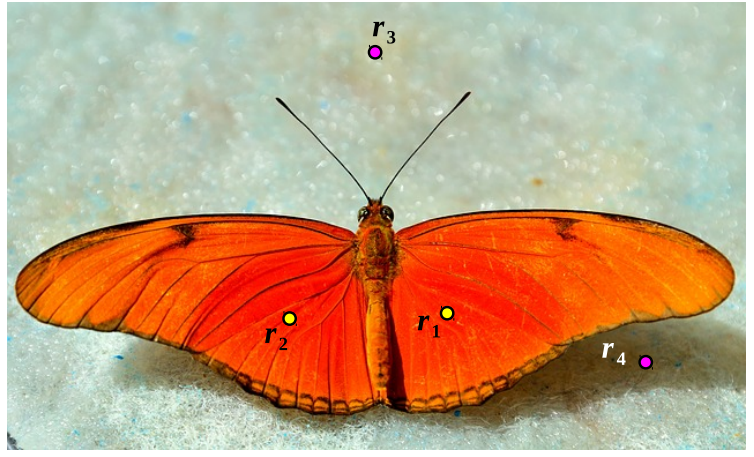

(a)

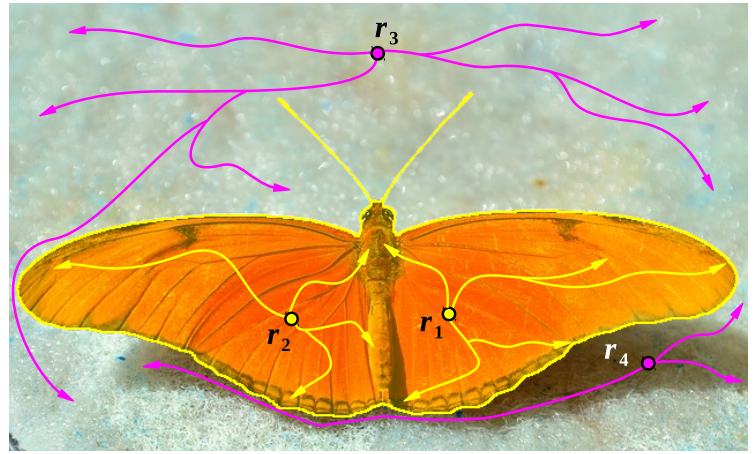

(b)

Figura 3.2: Exemplo de segmentação via IFT usando $f_{\max }$ : (a) Uma imagem com dois conjuntos de sementes (marcadores), tal que o conjunto de sementes internas $\mathcal{S}_{1}=\left\{r_{1}, r_{2}\right\}$ (marcadores internos) representa o objeto e o conjunto de sementes externas $\mathcal{S}_{0}=\left\{r_{3}, r_{4}\right\}$ (marcadores externos) o fundo. (b) Resultado da segmentação mostrando duas florestas de caminhos ótimos enraizadas nas sementes internas e externas, identificando o objeto e fundo respectivamente.

\begin{tabular}{|c|c|c|c|c|}
\hline 0 & 30 & 140 & 180 & 220 \\
\hline 40 & 90 & 120 & 210 & 190 \\
\hline 0 & 60 & 140 & 180 & 230 \\
\hline 40 & 100 & 70 & 140 & 190 \\
\hline 0 & 30 & 110 & 180 & 210 \\
\hline
\end{tabular}

(a)

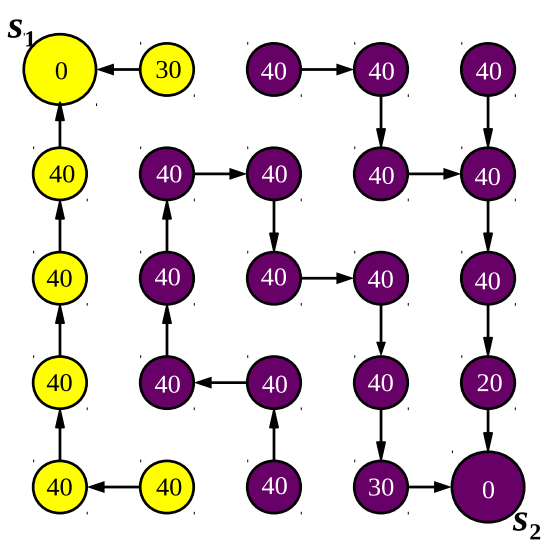

(c)

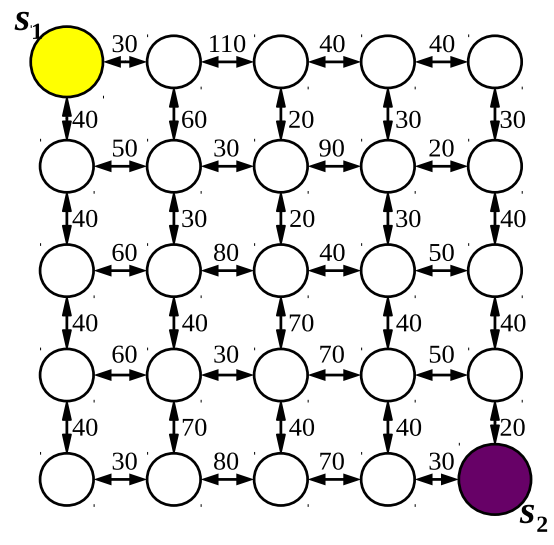

(b)

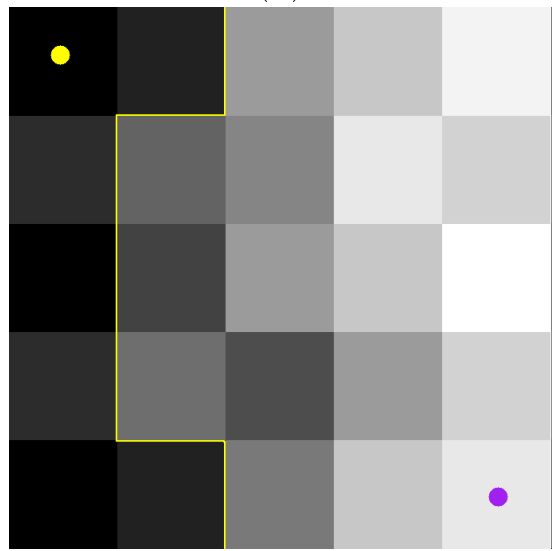

(d)

Figura 3.3: Exemplo de segmentação pela IFT com a função $f_{\max }$ : (a) Uma imagem de entrada $\hat{I}$ com dois marcadores escolhidos pelo usuário. Os quadros representam os pixels da imagem e os valores dentro deles as suas intensidades. (b) Um digrafo $G$, obtido a partir da imagem $\hat{I}$, com adjacência 4-conexo e conjunto de sementes $\mathcal{S}=\left\{s_{1}, s_{2}\right\}$. Os números ao lado das setas duplamente direcionadas (arcos simétricos igualmente ponderados) representam os pesos $\omega_{a}$ associados aos arcos. (c) Floresta de espalhamento $P$. As setas indicam o mapa de predecessores. Os números exibidos dentro dos nós representam os valores do mapa $V$ de custos. (d) A fronteira de separação entre as florestas ótimas enraizadas nas sementes $s_{1}$ e $s_{2}$. 


\subsubsection{Algoritmo da IFT}

O algoritmo da IFT (Algoritmo 1) segue essencialmente o procedimento do algoritmo de Dijkstra para o cálculo de caminhos de custo mínimo [Ahuja et al. (1993); Dijkstra (1959)], sendo ligeiramente modificado para permitir fontes múltiplas e uma função de custo mais geral.

\section{Algoritmo 1 - Algoritmo DA IFT}

Entrada: $\quad$ Imagem $\hat{I}=\langle\mathcal{I}, \vec{I}\rangle$, adjacência $\mathcal{A}$, conjuntos de sementes $\mathcal{S}_{\mathbf{1}}$ e $\mathcal{S}_{\mathbf{0}}$ e função de custo $f$. SAídA: $\quad$ Uma tripla $\langle L, P, V\rangle$ contendo o mapa de rótulos $L$, a floresta de espalhamento $P$ e o mapa de custos de caminho $V: \mathcal{I} \rightarrow \boldsymbol{F}$, tal que $V(t)=f\left(\pi_{t}^{P}\right)$, o qual converge para $V_{\text {opt }}^{f}$ (Equação 3.6) no caso de funções de custo MI.

Auxiliares: $\quad$ Fila $\mathcal{Q}$ de prioridade, variável tmp e um mapa de estado do vértice.

1. Para cada $t \in \mathcal{S}_{1}$, faça $L(t) \leftarrow 1$

2. Para cada $t \in \mathcal{S}_{0}$, faça $L(t) \leftarrow 0$

3. Para cada $t \in \mathcal{I}$, faça

4. $\quad P(t) \leftarrow$ nil e $V(t) \leftarrow f(\langle t\rangle)$.

5. $\quad \operatorname{estado}(t) \leftarrow 0$.

6. $\quad$ Se $V(t) \neq+\infty$, então insira $t$ em $\mathcal{Q}$.

7. Enquanto $\mathcal{Q} \neq \varnothing$, faça

8. $\quad$ Remova $s$ de $\mathcal{Q}$ cujo valor $V(s)$ é minimo.

9. $\quad \operatorname{estado}(s) \leftarrow 1$.

10. $\quad$ Para cada $\langle s, t\rangle \in \mathcal{A}$, tal que estado $(t)=0$, faça

11. $\quad$ Calcule tmp $\leftarrow f\left(\pi_{s}^{P} \cdot\langle s, t\rangle\right)$.

12. $\quad$ Se tmp $<V(t)$, então

13. $\quad \quad$ Se $V(t) \neq+\infty$, então remova $t$ de $\mathcal{Q}$.

14. $[$ Faça $P(t) \leftarrow s, V(t) \leftarrow t m p, L(t) \leftarrow L(s)$ e insira t em $\mathcal{Q}$.

Retorne $\langle L, P, V\rangle$

O Algoritmo 1 define os rótulos como $L(t)=1$ para todo $t \in \mathcal{S}_{\mathbf{1}}$ e $L(t)=0$ para todo $t \in \mathcal{S}_{\mathbf{0}}$ (Linhas 1 e 2). Nas Linhas 3 a 5 , para todos os caminhos triviais são inicializados os mapas de predecessores $(P)$, de custos $(V)$ e de estado do vértice. Na Linha 6 , todos os candidatos a raízes (sementes) são inseridos na fila $\mathcal{Q}$.

No caso de funções MI, o laço principal (Linhas 7 a 14) calcula os caminhos ótimos, de modo que em cada iteração um caminho $\pi_{s}^{P}$ de valor mínimo é obtido em $P$ quando removemos seu último vértice $s$ de $\mathcal{Q}$ (Linha 8). As linhas restantes (Linhas 9 a 14) calculam e propagam caminhos melhores para os vértices adjacentes, aplicando a seguinte regra de expansão: Se $f\left(\pi_{s}^{P} \cdot\langle s, t\rangle\right)<f\left(\pi_{t}^{P}\right)$ (Linha 12), então $\pi_{t}^{P}$ é trocado por $\pi_{s}^{P} \cdot\langle s, t\rangle$ (Linha 14), ou seja, o algoritmo avalia se o caminho $\pi_{s}^{P} \cdot\langle s, t\rangle$ que alcança $t$ por meio de $s$ é mais barato do que o caminho atual $\pi_{t}^{P}$ em $P$ e atualiza $P(t), V(t)$, $L(t)$ e $\mathcal{Q}$ adequadamente. No caso de funções não MI, uma floresta de espalhamento é calculada em $P$, porém o valor de custo calculado $V(t)$ pode diferir de $V_{o p t}^{f}(t)$.

\subsubsection{Fila de prioridade}

Seja $G=\langle\mathcal{I}, \mathcal{A}, \omega\rangle$ um digrafo com um número de vértices denotado por $n_{v}=|\mathcal{I}|$ e um número de arcos dado por $n_{a}=|\mathcal{A}|$.

O algoritmo da IFT (Algoritmo 1) usa uma fila de prioridade $\mathcal{Q}$ para propagar os rótulos das sementes para todos vértices do digrafo $G$, sendo também possível a propagação de outras 
informações para cada vértice, como sua ordem de propagação, uma medida de dissimilaridade, entre outros. A fila de prioridade contém os vértices que se encontram nas bordas das regiões em crescimento de cada semente, cuja propagação segue uma ordem não decrescente de valores de custo de caminho para o caso de funções MI.

O algoritmo da IFT terá uma complexidade de tempo $O\left(\left(n_{a}+n_{v}\right) \log n_{v}\right)$ usando um heap binário para a fila de prioridade $\mathcal{Q}$ e uma complexidade de $O\left(n_{a}+n_{v} k\right)$ se a fila $\mathcal{Q}$ usa bucket sort. Porém, para um digrafo $G$ esparso o algoritmo da IFT terá uma complexidade de $O\left(n_{v} \log n_{v}\right)$, quando a fila $\mathcal{Q}$ usa um heap binário e uma complexidade proporcional aos número de vértices, isto é $O\left(n_{v} k\right)$, se a fila $\mathcal{Q}$ usa bucket sort [Falcão et al. (2000)].

O caso em que a fila $\mathcal{Q}$ usa uma estrutura de bucket só é possível ao usar funções de custo de caminho com incrementos de custo $f\left(\pi_{s} \cdot\langle s, t\rangle\right)-f\left(\pi_{s}\right)$ inteiros, os quais estão limitados por uma constante $k$, tal que a propriedade $V_{\max }-V_{\min } \leqslant k$ deve ser mantida para preservar a corretude da fila, sendo $V_{\min }$ e $V_{\max }$ os valores mínimo e máximo de custo, respectivamente, dos vértices armazenados em $\mathcal{Q}$. A estrutura de bucket pode ser representada por uma fila circular com $k+1$ posições, na qual cada posição deve armazenar uma lista duplamente ligada de todos os vértices $t$ de mesmo custo, isto é, os vértices $t$ são inseridos no bucket $V(t) \%(k+1)$.

\subsubsection{Zonas de empate}

A floresta de caminhos ótimos calculada pela IFT, pode não ser única, o que leva a um problema de ambiguidade na segmentação resultante [Falcão et al. (2004)].

$\mathrm{O}$ problema de ambiguidade surge quando existe um vértice $t \in \mathcal{I}$ que pode ser alcançado a partir de duas ou mais sementes (raízes) com o mesmo custo mínimo e rótulos diferentes, conhecido como vértice de empate (Definição 9). Empates entre caminhos a partir de sementes diferentes com o mesmo rótulo não representam zonas de empate (Definição 10) reais, dado que levam ao mesmo resultado da segmentação [Miranda e Falcão (2009)].

Definição 9 (Vértice de empate) Para dados dois conjuntos de sementes $\mathcal{S}_{1}$ e $\mathcal{S}_{\mathbf{0}}$ representando diferentes classes (exemplo: objeto e fundo), um vértice $t$ é um vértice de empate se existem dois caminhos hereditariamente ótimos $\pi_{t}$ e $\tau_{t}$ tal que $R\left(\pi_{t}\right) \in \mathcal{S}_{0}$ e $R\left(\tau_{t}\right) \in \mathcal{S}_{1}$ (Figura 3.4).

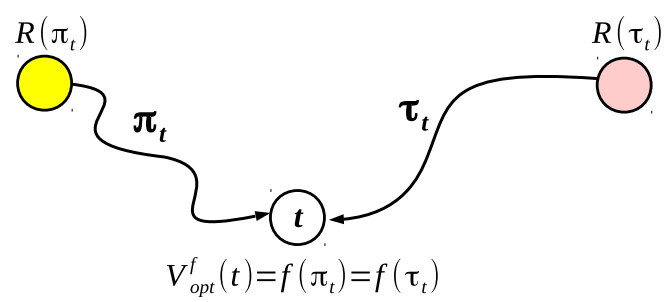

Figura 3.4: Exemplo de um vértice de empate t, em que $\pi_{t}$ e $\tau_{t}$ são dois caminhos hereditariamente ótimos a partir de sementes diferentes $R\left(\pi_{t}\right) \in \mathcal{S}_{1}$ e $R\left(\tau_{t}\right) \in \mathcal{S}_{0}$ respectivamente.

Definição 10 (Zona de empate) Uma zona de empate é um componente conexo maximal composto por vértices de empate, que forma uma subárvore em alguma floresta de caminhos ótimos (Figura 3.5). 


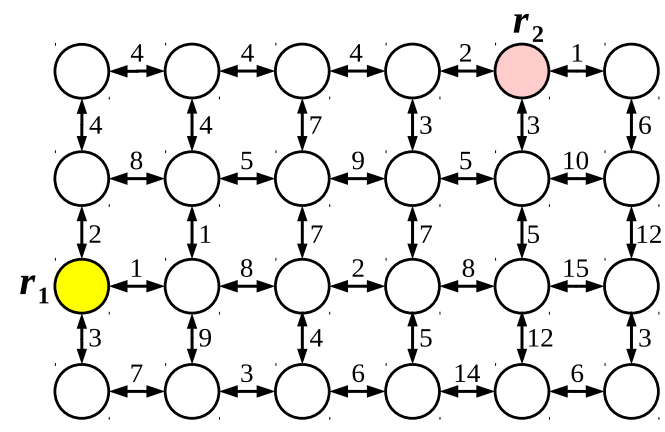

(a) $G$

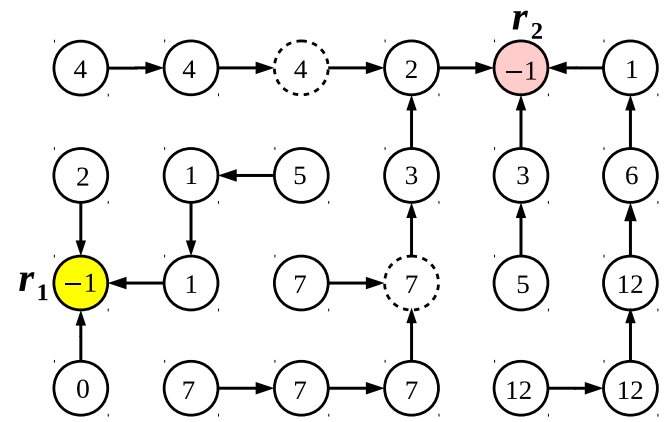

(c) $P_{2}$

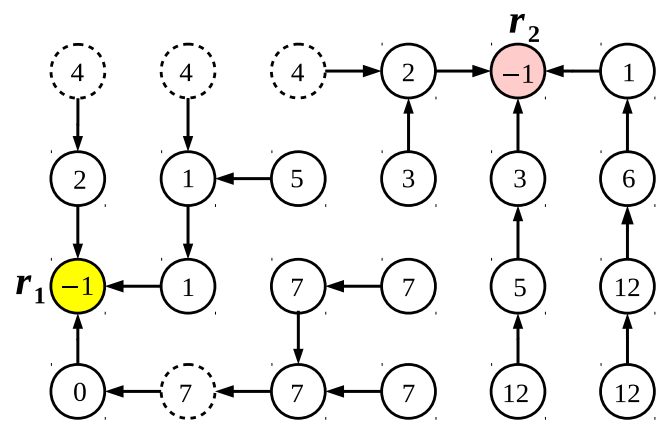

(b) $P_{1}$

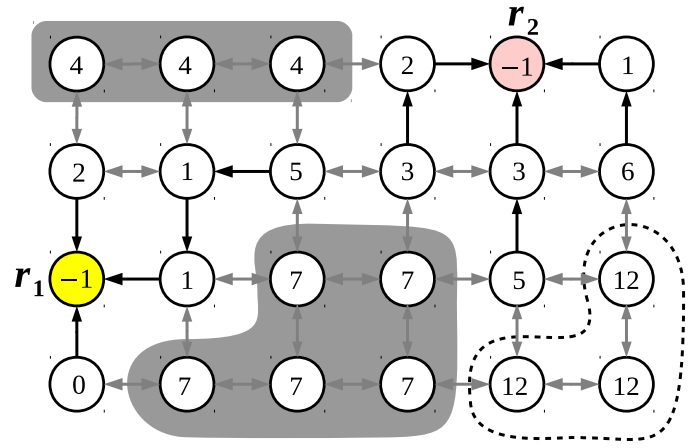

(d)

Figura 3.5: Exemplo de zonas de empate: (a) Um digrafo $G$ de vizinhança-4 com uma semente $r_{1}$ que representa o objeto e uma semente $r_{2}$ representando o fundo. Os números representam os pesos $\omega_{a}$ associados aos arcos. Em (b) e (c) são mostradas duas possiveis florestas de caminhos ótimos $P_{1}$ e $P_{2}$ usando a função de custo $f_{\max }$ (Equação 3.1). Os números dentro dos vértices indicam os valores de custo dos caminhos ótimos (Equação 3.6). Os vértices com contornos pontilhados são raizes de subárvores de caminhos ótimos compostos por vértices de empate. Em (d) duas zonas de empate (regiões em cinza) com valores de 4 e 7 são identificadas. Note que a região com borda pontilhada não representa uma zona de empate real, uma vez que qualquer caminho hereditariamente ótimo que conquiste os vértices dentro dessa região tem como origem a mesma semente $r_{2}$, levando a um mesmo resultado de segmentação. 


\subsubsection{Políticas de desempate}

O problema de ambiguidade das florestas ótimas pode ser solucionado realizando uma partição adequada das zonas de empate. A seguir são apresentadas três possíveis soluções de desempate.

\section{Política FIFO (First-In-First-Out)}

O algoritmo da IFT (Algoritmo 1, pág. 27) com política FIFO resolve o problema de ambiguidade das florestas em duas partes. Na primeira, o algoritmo da IFT decide sempre pelo primeiro caminho com menor custo que alcança um dado vértice, impedindo de atribuí-lo a qualquer outro caminho com mesmo custo que o alcance posteriormente (Linha 12). Na segunda parte, a fila de prioridade $\mathcal{Q}$ segue uma política de desempate FIFO escolhendo o primeiro pixel que entrou nela com mesmo custo.

Consequentemente, em uma política FIFO, uma zona de empate tende a ser dividida igualmente entre as sementes que a alcançam (Figura 3.6d).

\section{Política LIFO (Last-In-First-Out)}

O algoritmo da IFT (Algoritmo 1, pág. 27) com política LIFO também resolve o problema de ambiguidade das florestas em duas partes. A diferença em relação a política FIFO está no fato que o algoritmo da IFT com LIFO decide sempre pelo último caminho com custo mínimo que alcança um dado pixel, o que requer a modificação da expressão $t m p<V(t)$ da Linha 12 para $t m p \leqslant V(t)$, e a fila de prioridade segue uma política de desempate LIFO tal que o último pixel que entrou em $Q$ é escolhido no caso de pixel com mesmo custo. Em uma política LIFO, uma zona de empate será atribuída a uma única semente que a alcança (Figura 3.6f).

\section{Regra de desempate definida na própria função de custo}

Uma outra solução de desempate é incorporar uma regra de desempate dentro da própria definição da função de custo. Por exemplo, para resolver os empates da função $f_{\max }$ em favor do fundo, podemos considerar a função de custo $f_{\max }^{b k g}$ apresentada em [Miranda e Mansilla (2014)], a qual é definida como:

$$
\begin{aligned}
f_{\max }^{b k g}(\langle t\rangle) & = \begin{cases}-1, & \text { se } t \in \mathcal{S}_{1} \cup \mathcal{S}_{\mathbf{0}} \\
+\infty, & \text { caso contrário }\end{cases} \\
f_{\max }^{b k g}\left(\pi_{r \sim s} \cdot\langle s, t\rangle\right) & = \begin{cases}\max \left\{f_{\max }^{b k g}\left(\pi_{r \sim s}\right), 2 \times \omega_{a}(\langle s, t\rangle)+1\right\}, & \text { se } r \in \mathcal{S}_{\mathbf{1}} \\
\max \left\{f_{\max }^{b k g}\left(\pi_{r \sim s}\right), 2 \times \omega_{a}(\langle s, t\rangle)\right\}, & \text { se } r \in \mathcal{S}_{\mathbf{0}}\end{cases}
\end{aligned}
$$

Note que para caminhos não triviais (quando os pesos são inteiros), o custo de $f_{\max }^{b k g}$ é um número ímpar para caminhos vindos de $\mathcal{S}_{1}$ e um número par para caminhos provenientes de $\mathcal{S}_{\mathbf{0}}$, evitando assim as ambiguidades entre os diferentes rótulos, sendo a preferência dada para as sementes do fundo $\left(\mathcal{S}_{\mathbf{0}}\right)$. Essa formulação, dada pela função $f_{\max }^{b k g}$, corresponde a uma codificação alternativa para o método Iterative Relative Fuzzy Connectedness (IRFC) [Ciesielski et al. (2011)].

Nas Figuras 3.6g,h podemos observar um exemplo de partição de uma zona de empate usando a função $f_{\max }^{b k g}$. 


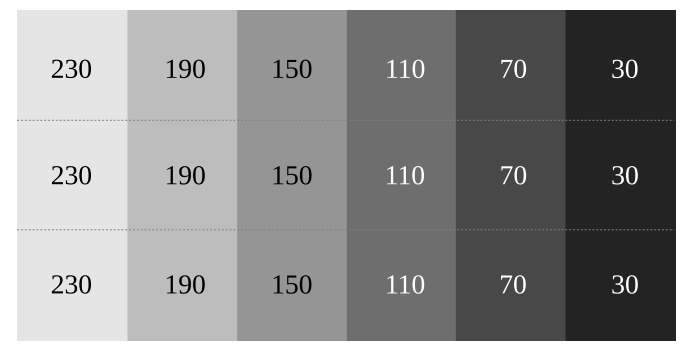

(a) $\hat{I}=\langle\mathcal{I}, I\rangle$

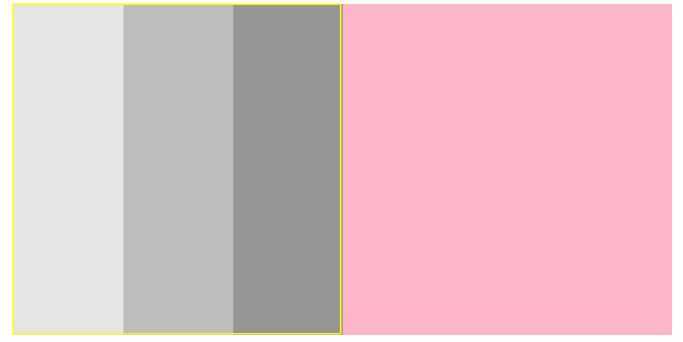

(c) $\mathcal{O}_{1}$

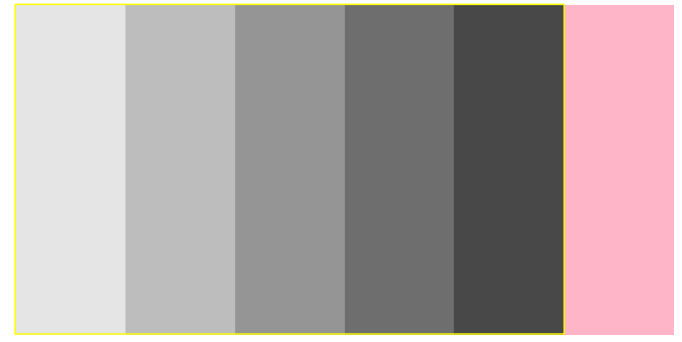

(e) $\mathcal{O}_{2}$

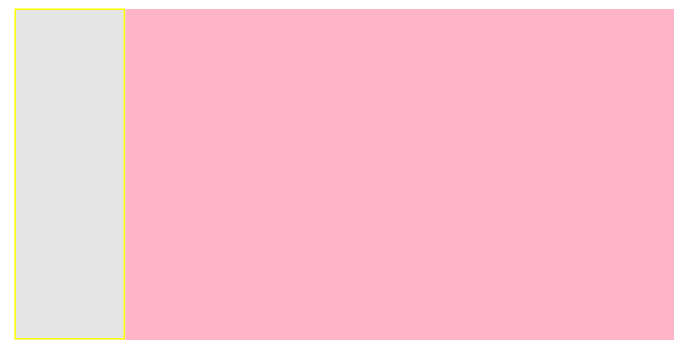

(g) $\mathcal{O}_{3}$

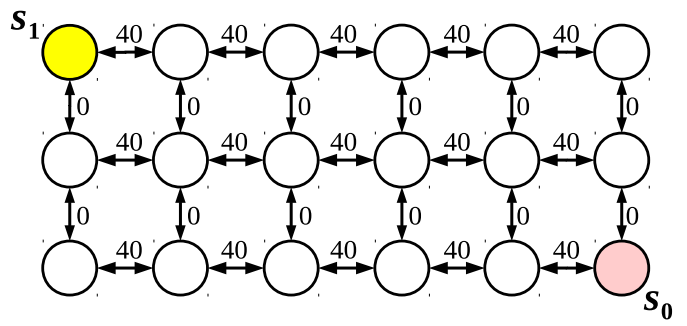

(b) $G=\langle\mathcal{I}, \mathcal{A}, \omega\rangle$
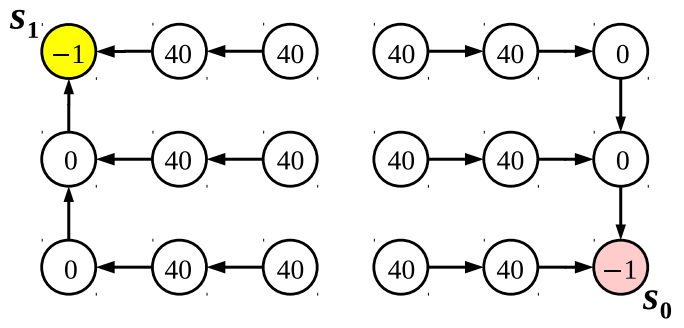

(d) $P_{1}$

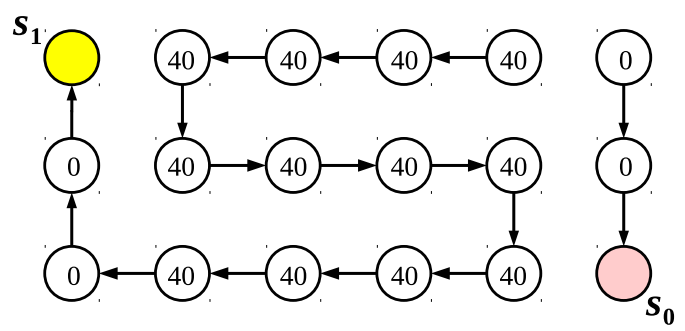

(f) $P_{2}$

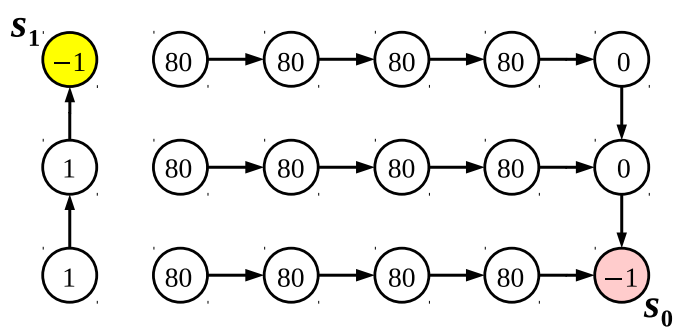

(h) $P_{3}$

Figura 3.6: Exemplo de políticas de desempate para o problema de ambiguidade das florestas. (a) Uma imagem em tons de cinza, em que cada quadro representa um pixel na imagem e os números representam as intensidades $I(t)$ dos pixels. (b) Um digrafo $G$ de vizinhança-4 derivado da imagem. As setas representam os arcos no digrafo e os números seus pesos associados $\omega_{a}$. As sementes $s_{1}$ e $s_{2}$ representam o objeto e fundo, respectivamente. Em (c) e (d) temos a imagem que representa o objeto $\mathcal{O}_{1}$ e a floresta $P_{1}$ resultantes da segmentação da IFT com $f_{\max }$ usando uma política de desempate FIFO. Em (e) e (f) temos a imagem que representa o objeto $\mathcal{O}_{2}$ e a floresta $P_{2}$ resultantes da segmentação da IFT com $f_{\max }$ usando uma política de desempate $L I F O$, no caso em que $\mathcal{S}_{1}$ é inserido após $\mathcal{S}_{\mathbf{0}}$ na fila $\mathcal{Q}$. Em (g) e (h) temos a imagem que representa o objeto $\mathcal{O}_{3}$ e a floresta $P_{3}$ resultantes da segmentação da IFT com $f_{\max }^{b k g}$ a qual possui uma regra de desempate na sua definição. 


\subsection{Condições suficientes para garantir a otimalidade dos caminhos da IFT}

No artigo original da IFT [Falcão et al. (2004)], uma classe de funções de custo foi apresentada, conhecidas como funções de conexidade suaves (Definição 11), as quais incluem as funções monotonicamente incrementais (MI) como um caso particular. Neste capítulo, provamos que as condições de suavidade apresentadas em [Falcão et al. (2004)] não são suficientes para garantir a otimalidade dos caminhos calculados pela IFT, tal como proposto em [Falcão et al. (2004)].

Definição 11 (Função de custo de caminho suave - PAMI2004) Uma função de custo de caminho $f$ é suave se para qualquer pixel $t \in \mathcal{I}$, existe um caminho ótimo $\pi_{t}$, o qual é ou trivial, ou tem a forma $\pi_{s} \cdot\langle s, t\rangle$ onde

(C1) $f\left(\pi_{s}\right) \leq f\left(\pi_{t}\right)$,

(C2) $\pi_{s}$ é ótimo, $e$

(C3) para qualquer caminho ótimo $\pi_{s}^{\prime}, f\left(\pi_{s}^{\prime} \cdot\langle s, t\rangle\right)=f\left(\pi_{t}\right)$.

Nesta seção, mostramos um contra-exemplo demonstrando que as condições C1-C3 da Definição 11 não são suficientes para garantir uma floresta de caminhos ótimos pelo algoritmo da IFT, bem como apresentamos um novo conjunto de condições que são suficientes para garantir a sua corretude, conforme publicado em [Condori et al. (2017)]. Paralelamente, essas inconsistências na formulação da IFT foram identificadas por um outro grupo de pesquisa, sendo que uma classe mais geral de funções com otimalidade garantida foi posteriormente apresentada em [Ciesielski et al. (2018)].

\subsubsection{Contra-exemplo para as condições de suavidade da IFT original (PAMI, 2004)}

Considere a função contra-exemplo $f_{C E}$ (Equação 3.14) e o digrafo de imagem da Figura 3.7.

$$
\begin{aligned}
f_{C E}\left(\pi_{t}=\langle t\rangle\right) & = \begin{cases}0, & \text { se } t \in \mathcal{S}, \\
+\infty, & \text { outro caso. }\end{cases} \\
f_{C E}\left(\pi_{t}=\pi_{s} \cdot\langle s, t\rangle\right) & = \begin{cases}8, & \text { se } \omega_{v}(s) \neq \omega_{v}(t), \\
4, & \text { se } \omega_{v}(s)=\omega_{v}(t) .\end{cases}
\end{aligned}
$$




\begin{tabular}{|c|c|c|c|c|c|}
\hline 255 & 255 & 255 & 255 & 255 & 255 \\
\hline 255 & 0 & 0 & 0 & 0 & 255 \\
\hline 255 & 255 & 255 & 255 & 255 & 255 \\
\hline
\end{tabular}

(a)

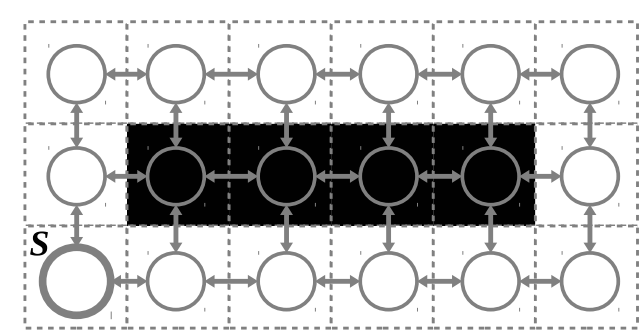

(b)

Figura 3.7: Contra-exemplo: (a) Uma imagem em que cada quadro representa um pixel e os valores dentro deles representam as suas intensidades. (b) Um digrafo derivado da imagem binária, no qual $\mathcal{S}$ é um conjunto de sementes contendo um único vértice e $\omega_{v}(t)=I(t)$ para todo pixel $t$.

Para esse digrafo, a função $f_{C E}$ satisfaz as condições de suavidade da Definição 11 (pág. 32). Na Figura 3.8 nós podemos observar que: Para qualquer pixel $t \in \mathcal{I} \backslash \mathcal{S}$, existe um caminho ótimo $\pi_{\mathcal{S} \sim t}$, tal que, $f_{C E}\left(\pi_{\mathcal{S} \sim s}\right) \leq f_{C E}\left(\pi_{\mathcal{S} \sim t}\right)(\mathrm{C} 1), \pi_{\mathcal{S} \sim s}$ é ótimo $(\mathrm{C} 2)$, e para qualquer caminho ótimo $\pi_{s}^{\prime}$ com destino em $s, f_{C E}\left(\pi_{s}^{\prime} \cdot\langle s, t\rangle\right)=f_{C E}\left(\pi_{\mathcal{S} \sim t}\right)$ (C3). Mas o algoritmo da IFT (Algoritmo 1, pág. 27) calcula uma floresta que não é ótima (Figura 3.9). Observe que, não existem soluções de florestas de caminhos ótimos para esse exemplo.

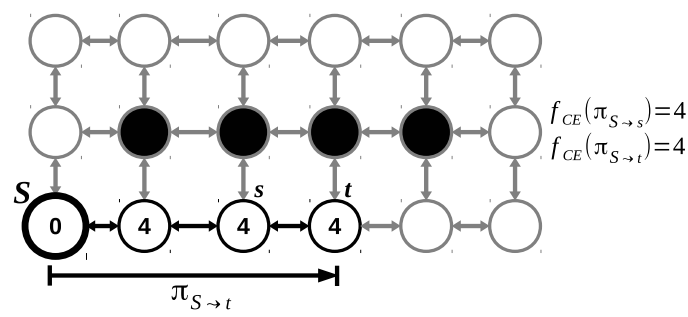

(a)

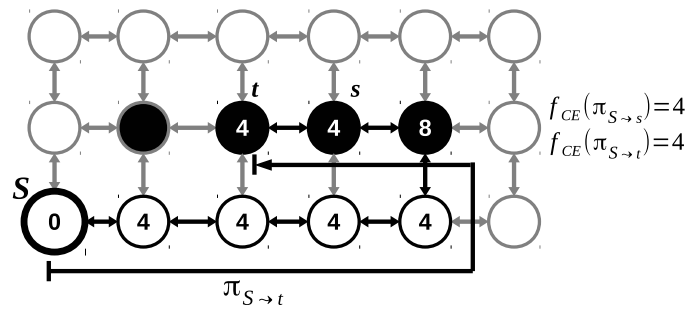

(c)

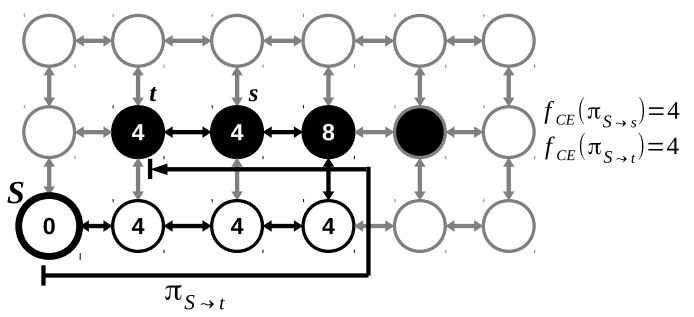

(b)

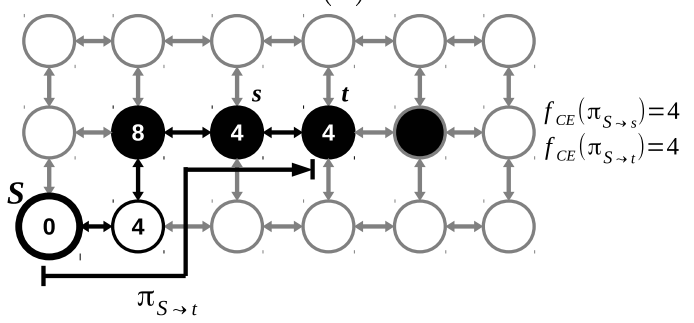

(d)

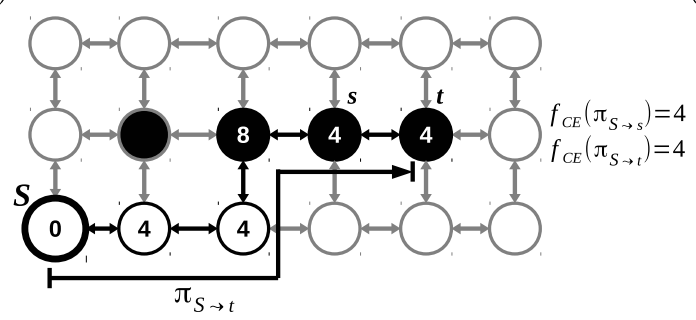

(e)

Figura 3.8: Contra-exemplo considerando $f_{C E}$ e o digrafo de imagem da Figura 3.7. A seta a partir da semente $\mathcal{S}$ para o nó $t$ representa um caminho ótimo $\pi_{\mathcal{S} \sim t}$, e os valores dentro dos nós representam os custos oferecidos pela semente em $\mathcal{S}$ a eles. (a) Caminhos não triviais $\pi_{\mathcal{S} \sim t}$ passando somente pelos nós brancos têm custo ótimo $V_{o p t}^{f_{C E}}(t)=4$. (b-e) Exemplos de caminhos ótimos $\pi_{\mathcal{S} \sim t}$ com $V_{o p t}^{f_{C E}}(t)=4$ para todos os nós pretos. Claramente, as condições da Definição 11 (pág. 32) são satisfeitas, mas não existe floresta ótima para esse digrafo usando a função $f_{C E}$. 


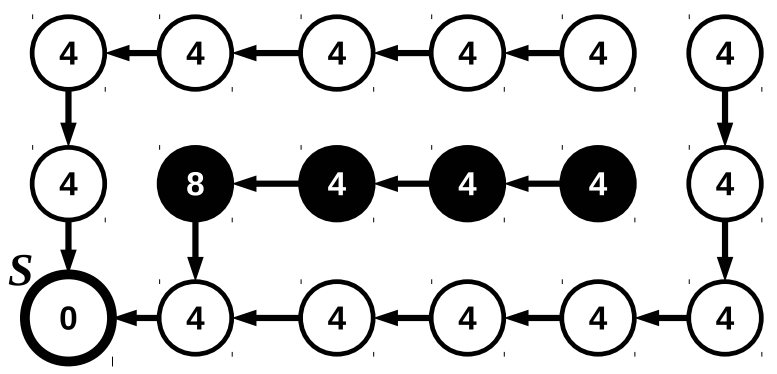

Figura 3.9: Contra-exemplo: Floresta calculada pela IFT usando $f_{C E}$ para o digrafo da imagem da Figura 3.7. Note que existe um nó com valor não ótimo $V(t)=8 \neq V_{o p t}^{f_{C E}}(t)=4$. Portanto a floresta resultante não é ótima.

Visando uma melhor compreensão do problema identificado, a seguir, é apresentada uma análise teórica da definição de suavidade da IFT original.

No Apêndice A de [Falcão et al. (2004)], é estabelecido que: "Por indução no comprimento de $\pi$, as Condições C1,C2, e C3 podem ser estendidas para prefixos arbitrários de caminhos ótimos", tal que as Condições $C 1, C 2$ e $C 3$ implicam nas Condições $C 1 *, C 2 *$ e $C 3 *$ dadas pela seguinte definição.

Definição 12 (Função de custo de caminho suave (prefixos arbitrários) - PAMI2004) Se $f$ é uma função de custo de caminho suave, então para qualquer $t \in \mathcal{I}$ existe um caminho ótimo $\pi$ para $t$, tal que, para quaisquer $\tau$ e $\lambda$ com $\pi=\tau \cdot \lambda$ temos

$(C 1 *) \quad f(\tau) \leq f(\pi)$,

$(C 2 *) \tau$ é ótimo,

$(C 3 *)$ para qualquer caminho ótimo $\tau^{\prime}$ com mesmo destino que $\tau, f\left(\tau^{\prime} \cdot \lambda\right)=f(\pi)$.

Considerando o contra-exemplo da Seção 3.3.1, é possível observar que $f_{C E}$ é uma função não suave de acordo com as Condições $C 1 *, C 2 *$ e $C 3 *$ da Definição 12 . Note que os caminhos representados nas Figuras 3.8b-3.8e não são ótimos para todos os prefixos, como requerido por $C 2 *$. Consequentemente, está claro que a Definição 11 (pág. 32) não implica na Definição 12 como estabelecido em [Falcão et al. (2004)], sendo essa conclusão errônea uma fonte de erro apresentada em [Falcão et al. (2004) $]^{2}$.

Consequentemente, uma análise mais profunda é necessária para generalizar a definição de suavidade.

\footnotetext{
${ }^{2}$ Posteriormente foi provado que até mesmo as Condições $C 1 *, C 2 *$ e $C 3 *$ da Definição 12 são insuficientes para garantir a otimalidade da IFT [Ciesielski et al. (2018)].
} 


\subsubsection{Versão suficiente das condições (ISMM, 2017)}

Motivados na busca de uma versão correta da IFT, propomos as seguintes Definições 13, 14 e 15), tal como descrito em [Condori et al. (2017)].

Definição 13 (Caminho hereditariamente ótimo) Um caminho $\pi_{t_{n}}=\left\langle t_{1}, t_{2}, \ldots, t_{n}\right\rangle$ é hereditariamente ótimo se todos os caminhos $\pi_{t_{i}}=\left\langle t_{1}, t_{2}, \ldots, t_{i}\right\rangle$ para $i=1,2, \ldots, n$ são caminhos ótimos.

Definição 14 (Função de caminho monotônica) Uma função $f$ é monotônica se para qualquer caminho $\pi_{t_{n}}=\left\langle t_{1}, t_{2}, \ldots, t_{n}\right\rangle$ no digrafo $G$ temos que $f\left(\left\langle t_{1}, \ldots, t_{i}\right\rangle\right) \leqslant f\left(\left\langle t_{1}, \ldots, t_{j}\right\rangle\right)$ sempre que $i \leqslant j \leqslant n$.

Definição 15 (Propriedade de substituição) Uma função $f$ tem a propriedade de substituição se para quaisquer caminhos $\pi_{s}$ e $\pi_{s}^{\prime}$ terminados em $s$, tal que $f\left(\pi_{s}\right)=f\left(\pi_{s}^{\prime}\right)$, temos que $f\left(\pi_{s} \cdot\langle s, t\rangle\right)=f\left(\pi_{s}^{\prime} \cdot\langle s, t\rangle\right)$, para qualquer $s \in \mathcal{I} \quad\langle\langle s, t\rangle \in \mathcal{A}$.

Assim, para uma dada função de custo de caminho $f$, que satisfaz as Definições 13,14 e 15, o algoritmo da IFT garante uma solução ótima de acordo com a Proposição 1 [Condori et al. (2017)], como demonstrado no Apêndice A.

Proposição 1 Para um dado digrafo $G=\langle\mathcal{I}, \mathcal{A}, \omega\rangle$, considere uma função de custo de caminho monotônica $f$ com a propriedade de substituição. Seja $\mathcal{O}$ o conjunto de todos os pixels $t \in \mathcal{I}$, tal que existe um caminho hereditariamente ótimo $\pi_{t}$ para $f$. Em qualquer floresta de espalhamento $P$ calculada pelo algoritmo da IFT para $f$, todos os caminhos $\tau_{t}^{P}$ com $t \in \mathcal{O}$ são caminhos ótimos.

Como consequência da Proposição 1, temos que para qualquer função de custo de caminho monotônica $f$ com a propriedade de substituição, o algoritmo da IFT (Algoritmo 1, pág. 27) só falha para encontrar uma floresta de caminhos ótimos $P$, se não existe tal floresta.

Note que em qualquer floresta de caminhos ótimos $P$, todos os caminhos $\pi_{t}^{P}$ são caminhos hereditariamente ótimos por definição. Portanto, a existência de uma floresta de caminhos ótimos $P$ implica que $\mathcal{O}=\mathcal{I}$ na Proposição 1 .

Posteriormente, condições menos restritas que garantem a otimalidade dos caminhos calculados pela IFT foram apresentadas em [Ciesielski et al. (2018)]. 


\subsection{Corte em grafo generalizado}

O Corte em Grafo Generalizado (GGC, Generalized Graph Cuts) [Ciesielski et al. (2012a,b); Couprie et al. (2010)] é um arcabouço que permite a descrição de diferentes métodos baseados em semente, tais como Random Walks [Grady (2006)], Graph Cuts [Boykov e Funka-Lea (2006)], Distance Cut [Bai e Sapiro (2007)], Iterative Relative Fuzzy Connectedness [Ciesielski et al. (2007)], e Watershed Cuts [Cousty et al. (2010)], cujos algoritmos têm o objetivo de encontrar uma segmentação que minimize uma função de energia de sua borda de corte.

\subsubsection{Funções de energia}

Sejam $G=\langle\mathcal{I}, \mathcal{A}, \omega\rangle$ um digrafo fortemente conexo derivado da imagem $\hat{I}$ e $\mathcal{U}$ o conjunto de todos os objetos $\mathcal{O}$ das segmentações possíveis da imagem $\hat{I}$.

No contexto de soluções de rotulação discretas, é possível distinguir duas classes importantes de energia, $\varepsilon_{1}$ e $\varepsilon_{\infty}$ [Ciesielski et al. (2012a)].

A energia $\varepsilon_{1}$ é definida como

$$
\varepsilon_{1}(\mathcal{O})=\sum_{\langle s, t\rangle \in \mathcal{C}(\mathcal{O})} \omega_{a}(\langle s, t\rangle)
$$

sendo que o peso $\omega_{a}(\langle s, t\rangle)$ representa uma medida de similaridade entre os vértices $s$ e $t$, tal que os pesos mais baixos identificam a borda de corte do objeto $\mathcal{O}$. Assim, a energia do objeto ótimo $\mathcal{O}_{\text {opt }} \in \mathcal{U}\left(\mathcal{S}_{1}, \mathcal{S}_{0}\right)$ deverá minimizar a energia $\varepsilon_{1}$ entre todas as possíveis soluções em $\mathcal{U}\left(\mathcal{S}_{1}, \mathcal{S}_{0}\right)$. A energia $\varepsilon_{1}$ pode ser otimizada usando o método do corte-mínimo/fluxo-máximo ( $\min$-cut/maxflow) [Boykov e Funka-Lea (2006)].

A energia $\varepsilon_{\infty}(\mathcal{O})$ pode ser definida na sua forma dual como:

$$
E(\mathcal{O})=\min _{\langle s, t\rangle \in \mathcal{C}(\mathcal{O})} \omega_{a}(\langle s, t\rangle)
$$

tal que o valor do peso $\omega_{a}(\langle s, t\rangle)$ representa nesse caso uma medida de dissimilaridade entre os vértices $s$ e $t$ (Figura 3.10).

Logo, o objeto de energia ótima $\mathcal{O}_{\text {opt }} \in \mathcal{U}\left(\mathcal{S}_{1}, \mathcal{S}_{\mathbf{0}}\right)$ deverá maximizar a energia $E$ entre todas as possíveis soluções em $\mathcal{U}\left(\mathcal{S}_{1}, \mathcal{S}_{0}\right)$, tal que $E\left(\mathcal{O}_{\text {opt }}\right) \geqslant E(\mathcal{O})$ para todo $\mathcal{O} \in \mathcal{U}\left(\mathcal{S}_{1}, \mathcal{S}_{\mathbf{0}}\right)$. Isto é,

$$
E\left(\mathcal{O}_{\text {opt }}\right)=\max _{\mathcal{O} \in \mathcal{U}\left(\mathcal{S}_{1}, \mathcal{S}_{0}\right)} E(\mathcal{O})
$$

No caso de digrafos simétricos, tais que $\omega_{a}(\langle s, t\rangle)=\omega_{a}(\langle t, s\rangle)$ para todo $\langle s, t\rangle \in \mathcal{A}$, a energia $E$ pode ser otimizada (maximizada) pela IFT usando a função MI $f_{\max }$ (Equação 3.1) (na ausência de zonas de empate ${ }^{3}$ ) e as funções não MI $f_{\max }^{b k g}$ (Equação 3.13) e $f_{\omega_{a}}$ (Equação 3.8), entre todas as segmentações possíveis satisfazendo as restrições de sementes (Figura 3.11).

\footnotetext{
${ }^{3}$ De modo mais geral, podemos também considerar as condições estabelecidas em [Miranda e Falcão (2009)].
} 


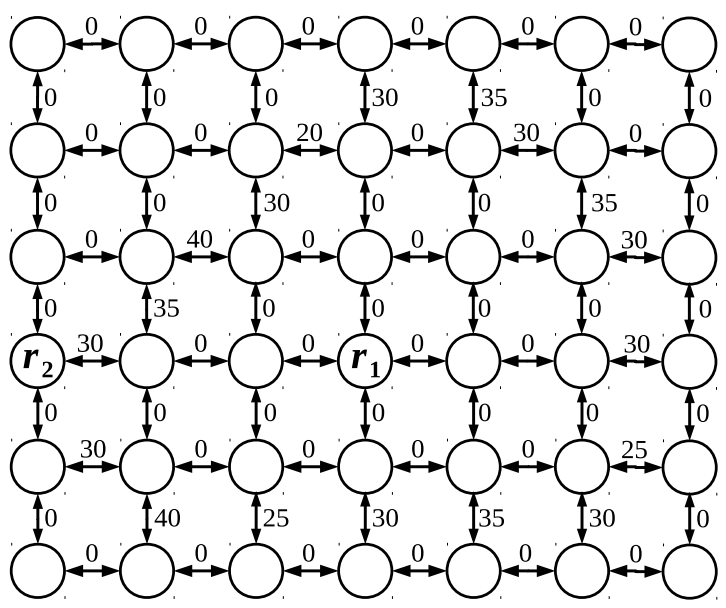

(a)

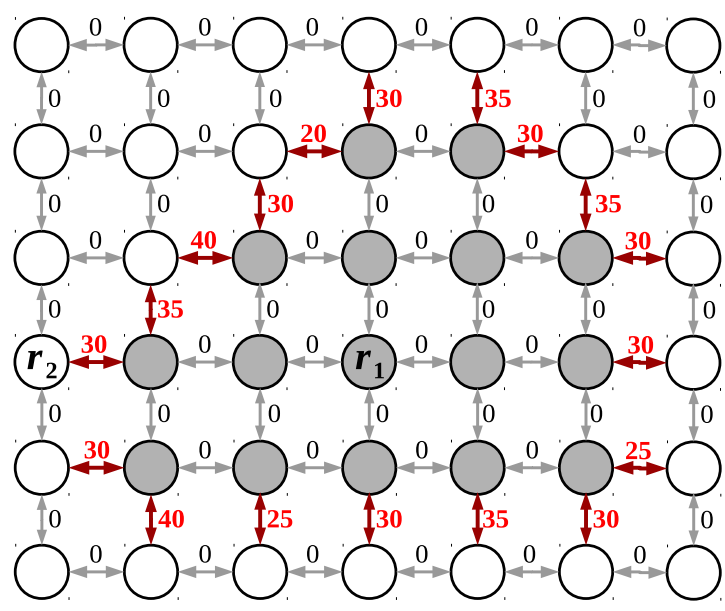

(b)

Figura 3.10: Exemplo de segmentação usando a função de energia $\varepsilon_{\infty}$ na sua forma dual. (a) Um grafo $G$ de vizinhança-4 com conjuntos de sementes $\mathcal{S}_{1}=\left\{r_{1}\right\}$ e $\mathcal{S}_{\mathbf{0}}=\left\{r_{2}\right\}$ representando o objeto e fundo, respectivamente. Os números nas setas representam os valores dos pesos $\omega_{a}$ associados aos arcos. (b) Um borda de corte do objeto é identificada com energia $E(\mathcal{O})=20$.

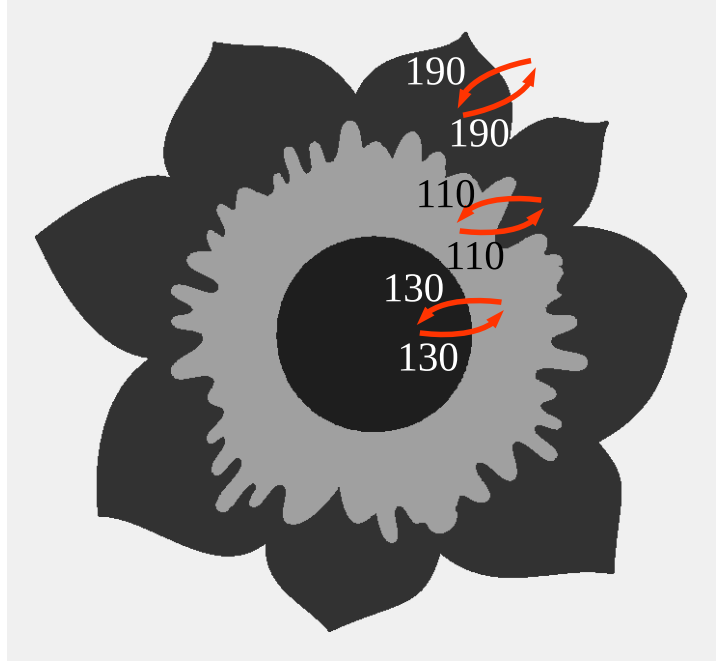

(a)

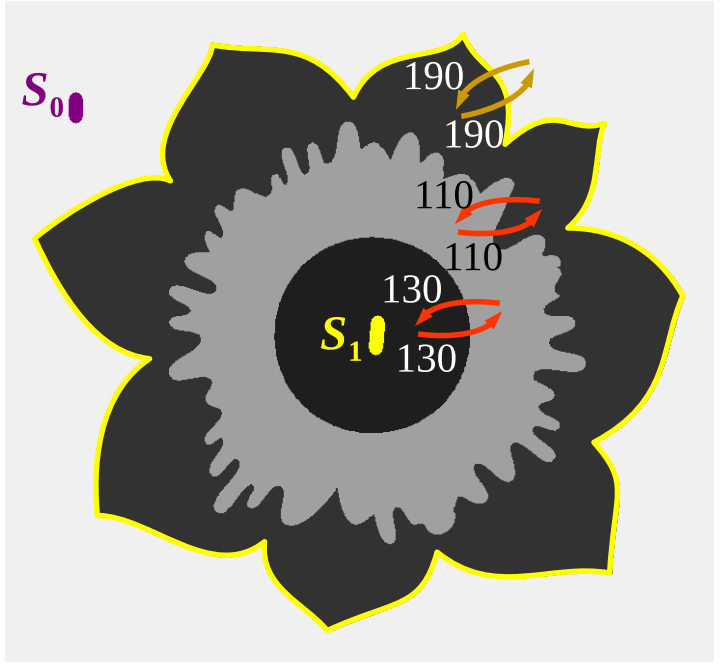

(b)

Figura 3.11: Exemplo de segmentação com maximização da função de energia E. (a) Representação esquemática de três possíveis bordas de corte, com alguns valores de arcos representativos $\omega_{a}(\langle s, t\rangle)$ indicados para cada região de segmento de borda. (b) Resultado da segmentação usando a função f fkg para as sementes mostradas $\left(\mathcal{S}_{\mathbf{1}}\right.$ e $\left.\mathcal{S}_{\mathbf{0}}\right)$. O segmento de borda com pesos de arcos $\omega_{a}(\langle s, t\rangle)$ igual a 190 tem preferência sobre os segmentos com pesos 130 e 110, devido à maximização da energia E (Equação 3.16) dada pelo mínimo peso na borda de corte. 


\subsection{Transformada Imagem-Floresta Orientada (OIFT)}

Seja $G$ um digrafo simétrico fortemente conexo (Subseções 2.2.4 e 2.2.9), no qual os pesos $\omega_{a}(\langle s, t\rangle)$ são uma combinação de uma medida comutativa de dissimilaridade $\delta_{a}(\langle s, t\rangle)$ entre pixels vizinhos $s$ e $t$, multiplicada por um fator de orientação, como a seguir:

$$
\omega_{a}(\langle s, t\rangle)= \begin{cases}\delta_{a}(\langle s, t\rangle) \times(1+\alpha), & \text { se } I(s)>I(t) \\ \delta_{a}(\langle s, t\rangle) \times(1-\alpha), & \text { se } I(s)<I(t) \\ \delta_{a}(\langle s, t\rangle), & \text { caso contrário }\end{cases}
$$

tal que $\alpha \in[-1,1]$. Diferentes procedimentos podem ser adotados para $\delta_{a}(\langle s, t\rangle)$, conforme discutido em [Ciesielski e Udupa (2010); Miranda et al. (2010)], tal como utilizar o valor absoluto da diferença das intensidades da imagem (isto é, $\delta_{a}(\langle s, t\rangle)=|I(s)-I(t)|$ ). Note que em geral te$\operatorname{mos} \omega_{a}(\langle s, t\rangle) \neq \omega_{a}(\langle t, s\rangle)$ quando $\alpha \neq 0$. Para imagens coloridas, um mapa de referência deve ser considerado para $I(t)$ na Equação 3.18, ou $\alpha$ deve ser ajustado para zero [Mansilla et al. (2013b)].

A Transformada Imagem-Floresta Orientada (OIFT, Oriented Image Foresting Transform) [Mansilla e Miranda (2013a); Miranda e Mansilla (2014)] é construída sobre o arcabouço da IFT considerando a seguinte função de custo de caminho em um digrafo simétrico:

$$
\begin{aligned}
f^{\sigma^{7}}(\langle t\rangle) & = \begin{cases}-1, & \text { se } t \in \mathcal{S}_{1} \cup \mathcal{S}_{0} \\
+\infty, & \text { caso contrário }\end{cases} \\
f^{\sigma^{\top}}\left(\pi_{r \sim s} \cdot\langle s, t\rangle\right) & = \begin{cases}\omega_{a}(\langle s, t\rangle), & \text { se } r \in \mathcal{S}_{1} \\
\omega_{a}(\langle t, s\rangle), & \text { caso contrário }\end{cases}
\end{aligned}
$$

na qual $\mathcal{S}_{\boldsymbol{1}}$ e $\mathcal{S}_{\boldsymbol{0}}$ denotam, respectivamente, os conjuntos de sementes selecionados dentro e fora do objeto a ser segmentado.

O objeto segmentado $\mathcal{O}^{\boldsymbol{P}}$ pela OIFT é definido a partir da floresta $P$ calculada pelo algoritmo da IFT (Algoritmo 1, pág. 27), com $f^{\sigma^{7}}$, tomando como pixels do objeto o conjunto de pixels que foram conquistados pelas raízes dos caminhos em $\mathcal{S}_{1}$, isto é, $\mathcal{O}^{\boldsymbol{P}}=\left\{t \in \mathcal{V} \mid R\left(\pi_{t}^{P}\right) \in \mathcal{S}_{\mathbf{1}}\right\}$. Para $\alpha>0$, a segmentação pela OIFT favorece transições a partir de pixels claros para pixels escuros e $\alpha<0$ favorece à orientação oposta.

A função $f^{0^{\top}}$ é uma função de conexidade não MI, como descrito em [Mansilla et al. (2013b)]. A otimalidade de $\mathcal{O}^{\boldsymbol{P}}$ pela OIFT é suportada por um critério de energia de corte em grafos envolvendo arcos a partir dos pixels do objeto para os pixels do fundo $\mathcal{C}\left(\mathcal{O}^{P}\right)$ (Equação 2.4), de acordo com o Teorema 1 de [Mansilla et al. (2013b); Miranda e Mansilla (2014)].

Teorema 1 (Otimalidade do corte pela OIFT) Para dados dois conjuntos de sementes $\mathcal{S}_{1} e$ $\mathcal{S}_{\mathbf{0}}$, seja $\mathcal{U}\left(\mathcal{S}_{1}, \mathcal{S}_{\mathbf{0}}\right)=\left\{\mathcal{O} \subseteq \mathcal{V} \mid \mathcal{S}_{\mathbf{1}} \subseteq \mathcal{O} \subseteq \mathcal{V} \backslash \mathcal{S}_{\mathbf{0}}\right\}$ o universo de todos os objetos possiveis satisfazendo as restrições de sementes. Qualquer floresta de espalhamento $P$ calculada pelo Algoritmo 1 para a função $f^{\sigma^{\top}}$ define um objeto segmentado $\mathcal{O}^{P}$ que maximiza $E$ (Equação 3.16) entre todos os resultados possíveis de segmentação em $\mathcal{U}\left(\mathcal{S}_{1}, \mathcal{S}_{0}\right)$. Isto é,

$$
E\left(\mathcal{O}^{P}\right)=\max _{\mathcal{O} \in \mathcal{U}\left(\mathcal{S}_{1}, \mathcal{S}_{0}\right)} E(\mathcal{O})
$$


Na Figura 3.12 podemos observar a segmentação da imagem da Figura 3.11a considerando $\alpha=0.5$, o que favorece a segmentação de um objeto com transições de pixels de claro para escuro.

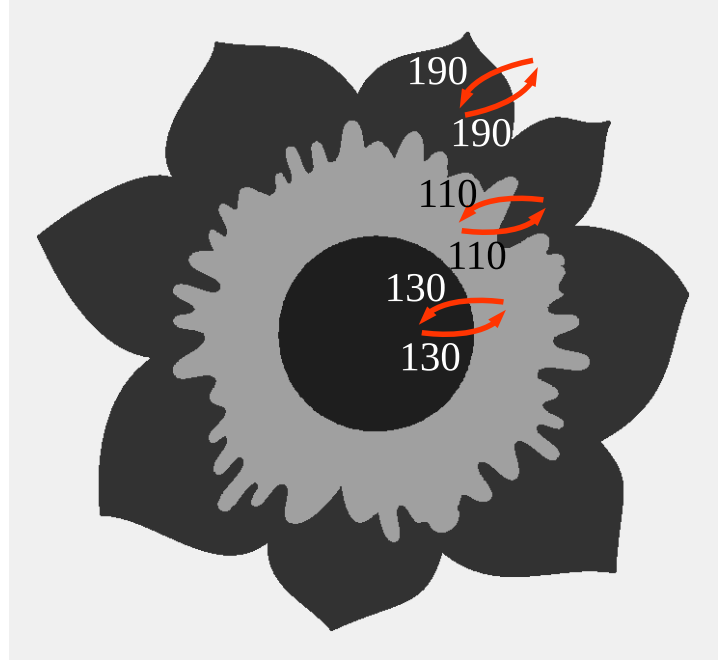

(a)

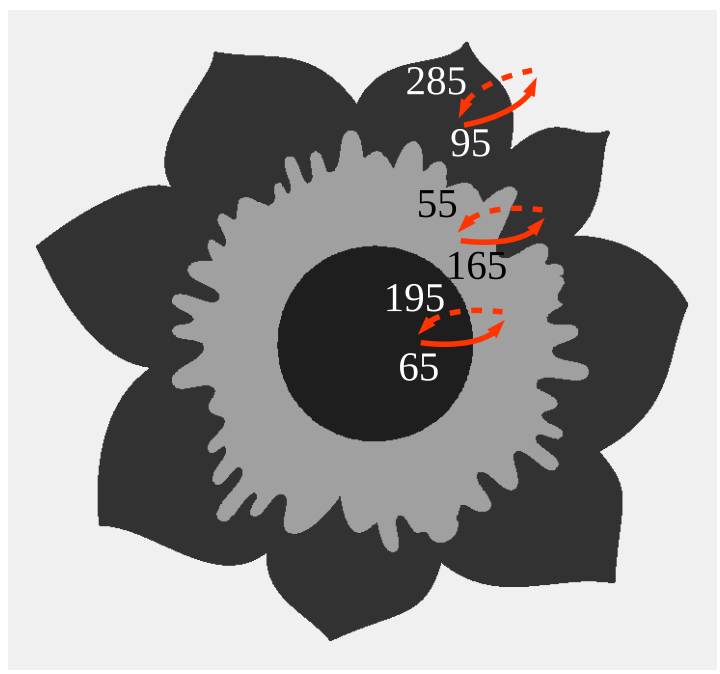

(b)

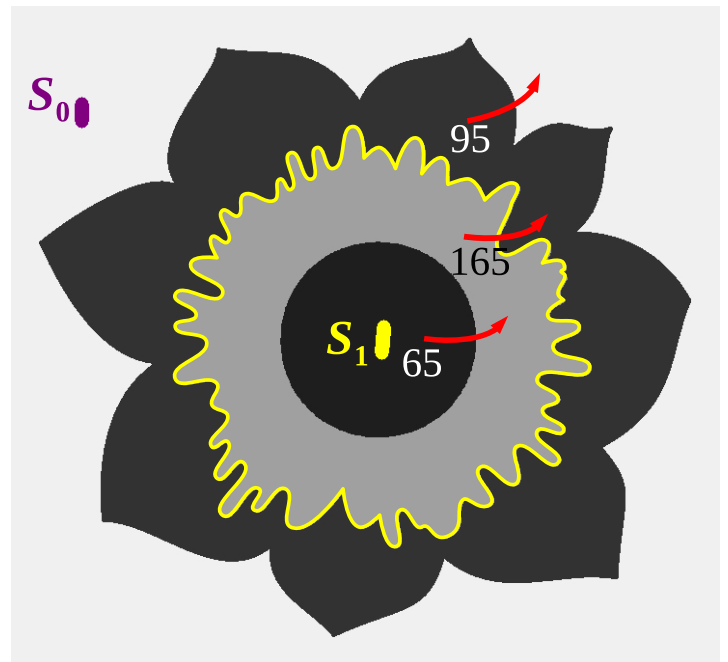

(c)

Figura 3.12: Exemplo de segmentação via OIFT. (a) Representação esquemática de três possíveis bordas de corte no digrafo da imagem, com alguns valores de arcos representativos $\delta_{a}(\langle s, t\rangle)$ indicados para cada região de segmento de borda. Em (b) são mostrados alguns valores de arcos representativos $\omega_{a}(\langle s, t\rangle), \alpha=0.5$, em que as setas contínuas representam os arcos pertencentes à borda de corte $\mathcal{C}(\mathcal{O})$ (Equação 2.4). (c) Resultado da segmentação usando a função $f^{0^{\lambda}}$ para as sementes $\mathcal{S}_{1}$ e $\mathcal{S}_{\mathbf{0}}$. O segmento de borda com pesos de arcos $\omega_{a}(\langle s, t\rangle)$ igual a 165 tem preferência sobre os segmentos com pesos 95 e 65 , devido à maximização da energia $E$ (Equação 3.16), isto é $E\left(\mathcal{O}_{\text {opt }}\right)=165$. 


\subsection{Riverbed}

Riverbed é um método de segmentação por perseguição de bordas que simula o comportamento da água que flui sobre um leito de rio (riverbed), procurando sempre níveis mais baixos do solo [Miranda et al. (2012)].

Seja $\left\langle s_{1}, s_{2}, \ldots, s_{\eta}\right\rangle$ uma sequência de sementes (pontos de ancoragem selecionados pelo usuário na borda do objeto), tal que $s_{1}=s_{\eta}$. Um contorno fechado com $\eta-1$ segmentos $\pi_{s_{1} \leadsto s_{2}}, \pi_{s_{2} \leadsto s_{3}}, \ldots, \pi_{s_{i-1} \leadsto s_{i}}, \ldots, \pi_{s_{\eta-1} \leadsto s_{\eta}}$ é calculado para a segmentação (Figura 3.13).

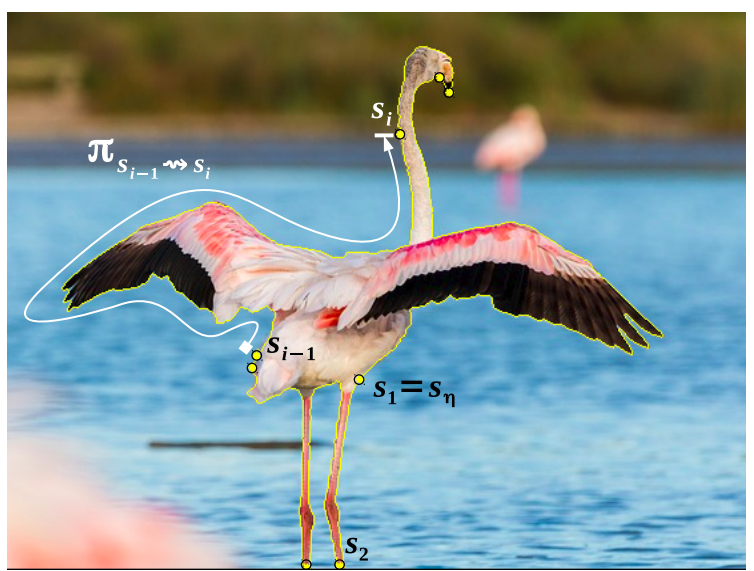

(a)

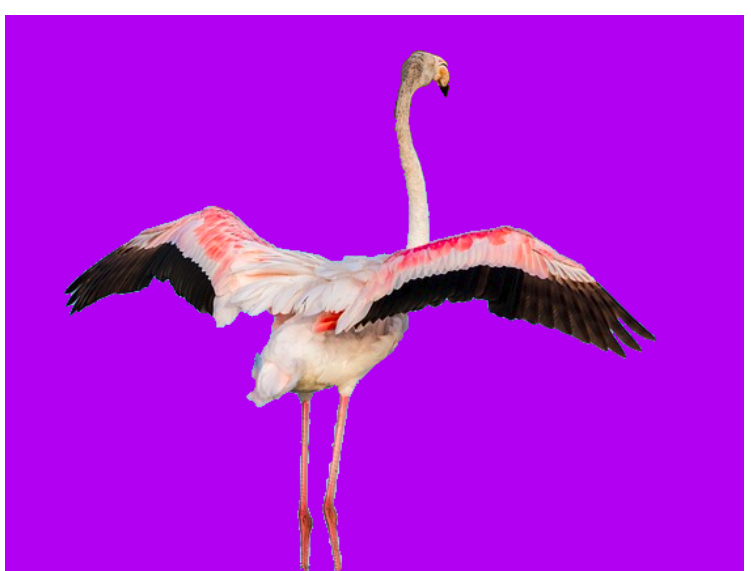

(b)

Figura 3.13: Exemplo de segmentação por Riverbed: (a) A sequência de pontos de ancoragem $\left\langle s_{1}, s_{2}, \ldots, s_{\eta}\right\rangle$ permite definir um contorno fechado (borda do objeto), e $\pi_{s_{i-1} \leadsto s_{i}}$ com $i=2, \ldots, \eta$ representa um caminho de energia ótima calculado pelo método com $f_{\text {river }}$. (b) Resultado da segmentação.

O método Riverbed resolve o problema de segmentação acima, calculando os segmentos $\pi_{s_{i-1} \leadsto s_{i}}$, $i=2, \ldots, n$, utilizando $\eta-1$ execuções do algoritmo da IFT [Falcão et al. (2004)] com a função $f_{\text {river }}$.

$$
\begin{aligned}
f_{\text {river }}(\langle t\rangle) & = \begin{cases}-1, & \text { se } t=s^{*} \\
+\infty, & \text { caso contrário }\end{cases} \\
f_{\text {river }}\left(\pi_{s} \cdot\langle s, t\rangle\right) & =\omega_{a}(\langle s, t\rangle)
\end{aligned}
$$

tal que $\omega_{a}(\langle s, t\rangle)$ possui valores baixos em regiões de elevado gradiente da imagem e $s^{*}=s_{i-1}$ é um ponto de início do segmento.

Conforme provado em [Miranda et al. (2012)], cada parte dos caminhos $\pi_{s_{i-1} \sim s_{i}}$ calculados pela

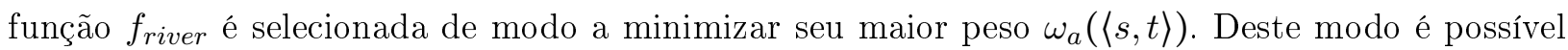
estabelecer uma relação teórica entre os caminhos de riverbed e a energia $\varepsilon_{\infty}$, usando um grafo dual no caso de grafos planares [Miranda et al. (2012)]. 


\section{Capítulo 4}

\section{Transformada Imagem-Floresta Orientada com restrições de conexidade}

Informações de alto nível sobre um dado objeto alvo, tais como sua forma, a polaridade da sua borda e se ele pode ser representado por um único componente conexo na imagem, representam conhecimentos prévios úteis para melhorar os resultados da sua segmentação. A restrição de conexidade de um objeto é uma importante propriedade topológica global que foi alvo de estudo em diferentes arcabouços para segmentação de imagens, em geral, resultando em um problema NPdifícil, tal como no algoritmo de corte-mínimo/fluxo-máximo [Vicente et al. (2008); Zeng et al. (2008)].

Neste capítulo apresentamos um novo método que incorpora restrições de conexidade na OIFT [Mansilla e Miranda (2013a); Miranda e Mansilla (2014)], chamado Transformada ImagemFloresta Orientada Conexa (COIFT, Connected Oriented Image Foresting Transform), que fornece soluções ótimas globais de acordo com a medida de corte em grafo $E$ (Equação 3.17) dentre todos resultados conexos possíveis, conservando o baixo custo computacional da OIFT (Figura 4.1), o qual foi publicado em [Mansilla et al. (2016)] e [Mansilla e Miranda (2016)].

Neste capítulo apresentamos de forma detalhada o método da COIFT, incluindo também uma análise sobre o tratamento de empates de energia.

\subsection{Restrição de conexidade}

Um objeto é conexo se para qualquer par de pontos no seu interior, existe uma curva de pontos os interligando, a qual está completamente contida no objeto (Figura 4.2).

A seguir apresentamos algumas definições consideradas numa versão discreta da restrição:

Definição 16 (Objeto conexo) Um objeto $\mathcal{O}$ é conexo se para qualquer par de vértices $p, q \in \mathcal{O}$ existe um caminho $\pi_{p \sim q}=\left\{t_{1}=p, t_{2}, \ldots, t_{i}, \ldots, t_{n}=q\right\}$, tal que $t_{i} \in \mathcal{O}, 1 \leqslant i \leqslant n$.

Definição 17 (Disco) Um disco $\mathcal{V}_{\odot}(c, \gamma)$, de centro $c$ e raio $\gamma$, é um conjunto de vértices $t \in \mathcal{I}$ definido por:

$$
\mathcal{V}_{\odot}(c, \gamma)=\{t \in \mathcal{I} \mid\|t-c\| \leq \gamma\}
$$

tal que $\|t-c\|$ é a distância Euclidiana entre os vértices t e c (Figura 4.3). 


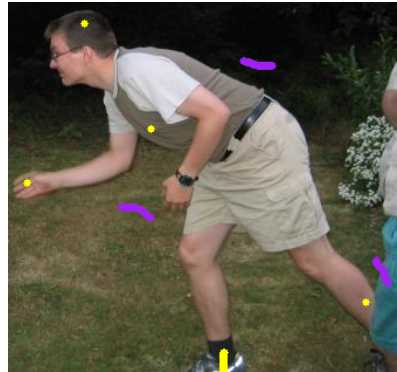

(a)

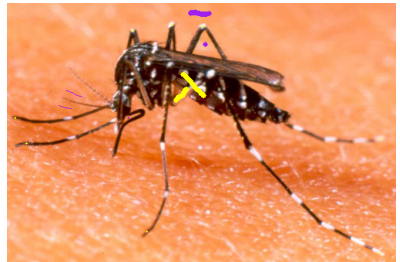

(d)

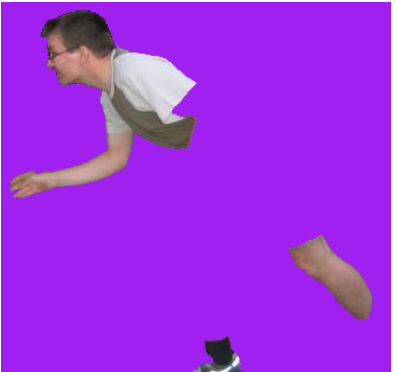

(b)

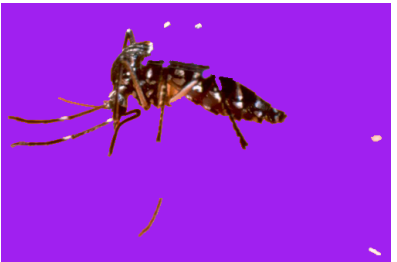

(e)

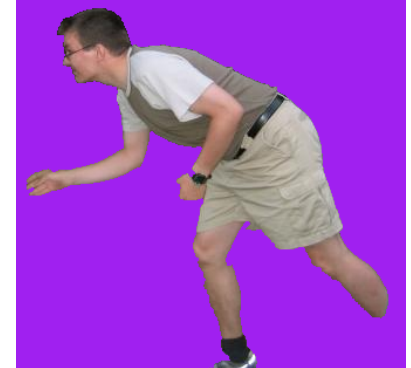

(c)

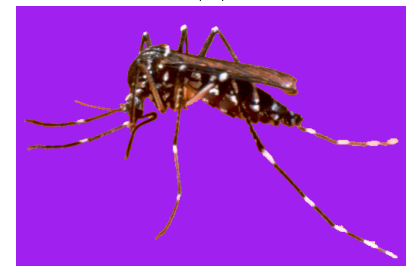

(f)

Figura 4.1: Exemplo da importância do uso da restrição de conexidade. (a,d) Imagens de entrada com marcadores selecionados pelo usuário. (b,e) Resultados da segmentação sem restrições de conexidade pela OIFT, gerando várias partes desconexas. (c,f) Os resultados propostos pela COIFT garantem objetos conexos a um baixo custo computacional.

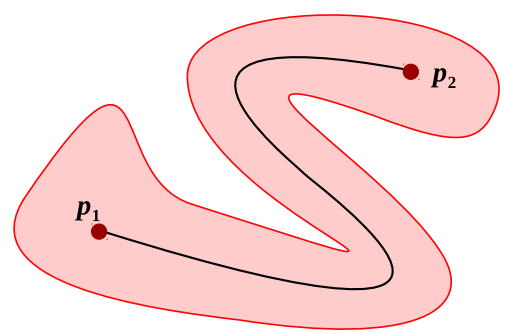

(a)

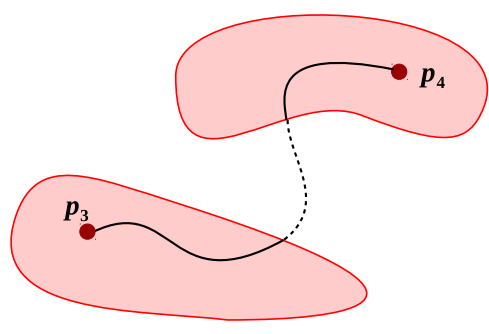

(b)

Figura 4.2: Exemplo de objetos conexos e não conexos. Em (a) um objeto conexo é mostrado, em que uma curva (linha em preto) contida completamente no objeto interliga os pontos $p_{1}$ e $p_{2}$. Em (b) temos que para os pontos $p_{3}$ e $p_{4}$ não existe uma curva contida completamente no objeto que os interligue, sendo portanto violada a restrição de conexidade.

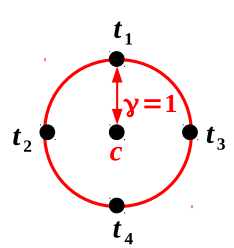

(a)

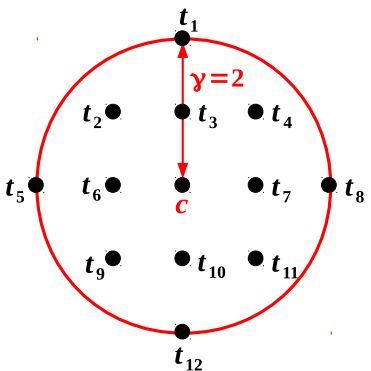

(b)

Figura 4.3: Exemplo de dois discos em um digrafo $G$. (a) Um disco $\mathcal{V}_{\odot}(c, \gamma=\mathbf{1})=\left\{c, t_{1}, t_{2}, t_{3}, t_{4}\right\}$. (b) Um disco $\mathcal{V}_{\odot}(c, \gamma=2)=\left\{c, t_{1}, t_{2}, t_{3}, t_{4}, t_{5}, t_{6}, t_{7}, t_{8}, t_{9}, t_{10}, t_{11}, t_{12}\right\}$. 
Definição 18 (Objeto $\gamma$-conexo) Para um dado objeto $\mathcal{O}$ e conjunto de sementes em seu interior $\mathcal{S}_{1}$, seja $\boldsymbol{R}^{\gamma}(\mathcal{O}){ }^{1}$ o conjunto de todos os vértices $t \in \mathcal{O}$, tal que $t$ é o centro de um disco com raio $\gamma\left(\mathcal{V}_{\odot}(t, \gamma)\right)$, o qual está completamente contido no objeto $\mathcal{O}$. Isto é:

$$
\boldsymbol{R}^{\gamma}(\mathcal{O})=\left\{t \in \mathcal{O} \mid \mathcal{V}_{\odot}(t, \gamma) \subseteq \mathcal{O}\right\}
$$

Um objeto $\mathcal{O}$ é $\gamma$-conexo em relação a $\mathcal{S}_{1}$ se as sementes em $\mathcal{S}_{1}$ estão todas em um mesmo componente conexo de $\boldsymbol{R}^{\gamma}(\mathcal{O})$. Isto é, para qualquer par de vértices $p, q \in \mathcal{S}_{1}$, existe um caminho $\pi_{p \sim q}=\left\{t_{1}=p, t_{2}, \ldots, t_{i}, \ldots, t_{n}=q\right\}$, tal que $t_{i} \in \boldsymbol{R}^{\gamma}(\mathcal{O}), 1 \leqslant i \leqslant n$ (Figura 4.4).

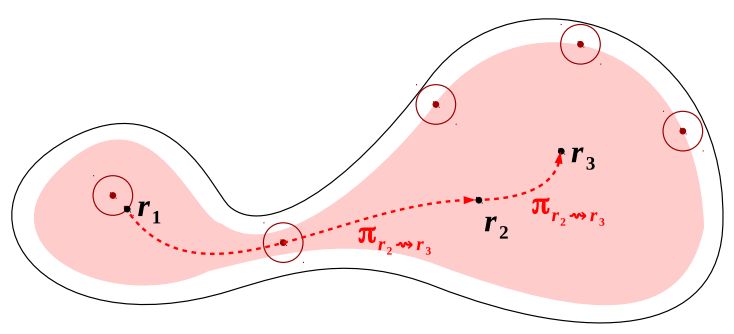

(a) Objeto 1-conexo em relação a $\left\{r_{1}, r_{2}, r_{3}\right\}$

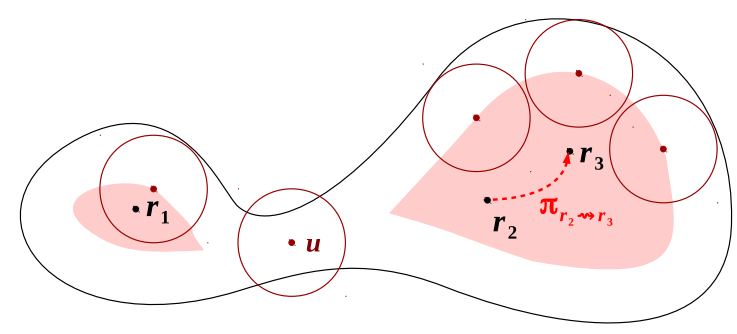

(b) Objeto não 3-conexo em relação a $\left\{r_{1}, r_{2}, r_{3}\right\}$

Figura 4.4: Exemplos de objetos $\gamma$-conexos e não $\gamma$-conexos. Para um objeto $\mathcal{O}$ com $\mathcal{S}_{1}=\left\{r_{1}, r_{2}, r_{3}\right\}$, temos em: (a) Uma região $\boldsymbol{R}^{\gamma}(\mathcal{O})$ com $\gamma=1$ (região sombreada) tal que $r_{1}, r_{2}$ e $r_{3}$ pertencem ao mesmo componente conexo de $\boldsymbol{R}^{\gamma}(\mathcal{O})$, identificando um objeto 1-conexo. (b) Uma região $\boldsymbol{R}^{\gamma}(\mathcal{O})$ com $\gamma=3$ (região sombreada) tal que $r_{1}$ não pertence ao mesmo componente conexo de $r_{2}$ e $r_{3}$ em $\boldsymbol{R}^{\gamma}(\mathcal{O})$, não satisfazendo-se a restrição de 3-conexidade. Note que para $\gamma=1$, os caminhos $\pi_{r_{1} \sim r_{2}}, \pi_{r_{2} \sim r_{3}}$ e $\pi_{r_{1} \sim r_{3}}=\pi_{r_{1} \sim r_{2}} \cdot \pi_{r_{2} \sim r_{3}}$ estão completamente contidos em $\boldsymbol{R}^{\gamma}(\mathcal{O})$ e para $\gamma=3$ não existem caminhos que interliguem $r_{1}$ com $r_{2}$ e $r_{1}$ com $r_{3}$ e que estejam completamente contidos em $\boldsymbol{R}^{\gamma}(\mathcal{O})$.

As seguintes propriedades podem ser verificadas:

1. Um objeto $\mathcal{O}$ é 0 -conexo em relação a $\mathcal{O}$ (isto é, $\mathcal{S}_{1}=\mathcal{O}$ ), se e somente se $\mathcal{O}$ é conexo.

2. Sejam $\beta, \theta \in \mathbb{R}$, tal que $\beta \leqslant \theta$, se um objeto $\mathcal{O}$ é $\theta$-conexo em relação a $\mathcal{S}_{1}$, então $\mathcal{O}$ é $\beta$-conexo em relação a $\mathcal{S}_{1}$.

Observe que, de acordo com a Propriedade 2, com o aumento do valor de $\gamma$ a restrição de objeto $\gamma$-conexo passa ser mais restritiva, reduzindo o universo de objetos que a satisfazem.

\subsection{Segmentação de um objeto conexo via COIFT}

Seja $\boldsymbol{A}$ um dado conjunto de pixels, o valor ótimo de energia usando o conjunto $\boldsymbol{A}$ como sementes internas é denotado por:

$$
E_{\boldsymbol{A}}=\max _{\mathcal{O} \in \mathcal{U}\left(\boldsymbol{A}, \mathcal{S}_{0}\right)} E(\mathcal{O})
$$

De acordo com essa mesma notação, $E_{\{t\}}$ denota a energia ótima a partir de uma única semente

\footnotetext{
${ }^{1}$ O conjunto $\boldsymbol{R}^{\gamma}(\mathcal{O})$ também pode ser interpretado como a erosão do objeto $\mathcal{O}$ com um elemento estruturante circular de raio $\gamma$.
} 
interna $t$, quando $\mathcal{S}_{1}=\{t\}$. Isto é:

$$
E_{\{t\}}=\max _{\mathcal{O} \in \mathcal{U}\left(\{\boldsymbol{t}\}, \mathcal{S}_{0}\right)} E(\mathcal{O})
$$

Para um conjunto de sementes $\boldsymbol{A} \cup \boldsymbol{B}$ a energia $E_{\boldsymbol{A} \cup \boldsymbol{B}}$ é sempre menor ou igual às energias das partes individuais $E_{\boldsymbol{A}}$ e $E_{\boldsymbol{B}}$. Isto é, $E_{\boldsymbol{A} \cup \boldsymbol{B}} \leq \min \left\{E_{\boldsymbol{A}}, E_{\boldsymbol{B}}\right\}$. Essa afirmação é uma consequência direta da otimização da energia do corte em grafo (Teorema 1, pág. 38), pois um corte ótimo num espaço pequeno de busca de possíveis bordas de corte com mais restrições (conjunto $\boldsymbol{A} \cup \boldsymbol{B}$ ) não pode gerar valores de energia melhores do que uma busca num espaço maior com poucas restrições $(\boldsymbol{A}$ ou $\boldsymbol{B})$. Assim, a borda ótima de corte para $\boldsymbol{A} \cup \boldsymbol{B}$ não pode ter uma energia maior do que a energia ótima obtida para $\boldsymbol{A}$ ou para $\boldsymbol{B}$.

A seguir, a Proposição 2, com prova no Apêndice B, dá uma caracterização ainda mais forte.

Proposição $2 A$ energia ótima $E_{\boldsymbol{A} \cup \boldsymbol{B}}$ dentre todos os objetos em $\mathcal{U}\left(\boldsymbol{A} \cup \boldsymbol{B}, \mathcal{S}_{\mathbf{0}}\right)$, satisfaz $E_{\boldsymbol{A} \cup \boldsymbol{B}}=\min \left\{E_{\boldsymbol{A}}, E_{\boldsymbol{B}}\right\}$.

Seja $\mathcal{O}_{\text {opt }}$ um objeto ótimo com energia máxima em $\mathcal{U}\left(\mathcal{S}_{1}, \mathcal{S}_{0}\right)$. A partir da Proposição 2, podemos concluir que

$$
E\left(\mathcal{O}_{\text {opt }}\right)=\min _{s \in \mathcal{S}_{1}} E_{\{s\}}
$$

De fato, também podemos deduzir que

$$
E\left(\mathcal{O}_{\text {opt }}\right)=\min _{t \in \mathcal{O}_{\text {opt }}} E_{\{t\}}
$$

uma vez que $\mathcal{O}_{\text {opt }} \in \mathcal{U}\left(\mathcal{O}_{\text {opt }}, \mathcal{S}_{0}\right) \subseteq \mathcal{U}\left(\mathcal{S}_{1}, \mathcal{S}_{0}\right)$ e $E\left(\mathcal{O}_{\text {opt }}\right)=\max _{\mathcal{O} \in \mathcal{U}\left(\mathcal{S}_{1}, \mathcal{S}_{0}\right)} E(\mathcal{O})$, o que implica que

$$
E\left(\mathcal{O}_{\text {opt }}\right)=\max _{\mathcal{O} \in \mathcal{U}\left(\mathcal{O}_{\text {opt }}, \mathcal{S}_{0}\right)} E(\mathcal{O})
$$

Seja $V_{o p t}^{f_{\max }}(t)$ o custo de um caminho ótimo até $t$ calculado pelo algoritmo da IFT com a função $f_{\max }^{\# \mathcal{S}_{0}}$ (Equação 4.8) usando um digrafo simétrico. A função $f_{\max }^{\# \mathcal{S}_{0}}$ é um caso particular da função $f_{\max }$ (Equação 3.1), que processa os pesos de arcos antiparalelos $\langle t, s\rangle$ ao longo do caminho com origem numa semente em $\mathcal{S}_{\mathbf{0}}$ (Figura 4.5).

$$
\begin{aligned}
f_{\max }^{\# \mathcal{S}_{0}}(\langle t\rangle) & = \begin{cases}-1, & \text { se } t \in \mathcal{S}_{\mathbf{0}} \\
+\infty, & \text { caso contrário }\end{cases} \\
f_{\max }^{\# \mathcal{S}_{0}}\left(\pi_{s} \cdot\langle s, t\rangle\right) & =\max \left\{f_{\max }^{\# \mathcal{S}_{0}}\left(\pi_{s}\right), \omega_{a}(\langle t, s\rangle)\right\}
\end{aligned}
$$




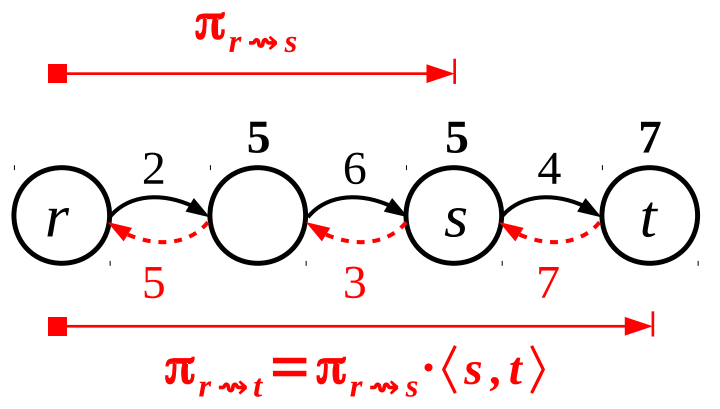

Figura 4.5: Exemplo de um caminho $\pi_{r \sim t}=\pi_{r \sim s} \cdot\langle s, t\rangle$ usando a função $f_{\max }^{\# \mathcal{S}_{0}}$, o qual processa pesos de arcos antiparalelos (setas pontilhadas). Os números nas setas representam os pesos associados aos arcos, $e$ os números sobre os vértices são os custos oferecidos pelo vértice raiz $r \in \mathcal{S}_{0}$, tal que $f_{\max }^{\# \mathcal{S}_{0}}\left(\pi_{r \sim s}\right)=5$ e $f_{\max }^{\# \mathcal{S}_{\mathrm{O}}}\left(\pi_{r \sim t}\right)=7$.

Um objeto ótimo $\mathcal{O}_{\text {opt }} \in \mathcal{U}\left(\{\boldsymbol{t}\}, \mathcal{S}_{\mathbf{0}}\right)$ possui uma energia máxima $E\left(\mathcal{O}_{\text {opt }}\right)=E_{\{t\}}$ equivalente ao valor de custo ótimo $V_{o p t}^{f_{\max } \mathcal{S}_{0}}(t)$, como definido na Proposição 3, a qual segue imediatamente do Lema 1 em [Bejar e Miranda (2015)] na sua forma dual.

Proposição 3 Para um dado digrafo simétrico fortemente conexo $G$ e conjuntos de sementes $\mathcal{S}_{\mathbf{1}}$ e $\mathcal{S}_{\mathbf{0}}$, tal que $\mathcal{S}_{1}=\{t\}$, nós temos que $E_{\{t\}}=V_{\text {opt }}^{f_{\max }^{f}}(t)$.

Assim, das Proposições 2 e 3 temos que um objeto ótimo $\mathcal{O}_{\text {opt }} \in \mathcal{U}\left(\mathcal{S}_{1}, \mathcal{S}_{\mathbf{0}}\right)$ possui uma energia máxima definida por:

$$
E\left(\mathcal{O}_{\text {opt }}\right)=\min _{t \in \mathcal{S}_{1}} E_{\{t\}}
$$

e

$$
E\left(\mathcal{O}_{\text {opt }}\right)=\min _{t \in \mathcal{S}_{1}} V_{o p t}^{f_{\max }}(t)
$$

Seja $\mathcal{U}_{\boldsymbol{c}}\left(\mathcal{S}_{1}, \mathcal{S}_{\mathbf{0}}\right)$ o conjunto de todos os objetos conexos satisfazendo as restrições de semente (isto é, $\mathcal{U}_{c}\left(\mathcal{S}_{1}, \mathcal{S}_{0}\right) \subseteq \mathcal{U}\left(\mathcal{S}_{1}, \mathcal{S}_{0}\right)$ ), definido por:

$$
\mathcal{U}_{c}\left(\mathcal{S}_{1}, \mathcal{S}_{0}\right)=\left\{\mathcal{O} \in \mathcal{U}\left(\mathcal{S}_{1}, \mathcal{S}_{0}\right) \mid G[\mathcal{O}] \text { é fortemente conexo }\right\}
$$

tal que $G[\mathcal{O}]$ é o subgrafo dirigido induzido por $\mathcal{O}$ (Subseção 2.2.5).

A COIFT maximiza a energia $E(\mathcal{O})$ (Equação 3.16) em um digrafo simétrico fortemente conexo $G$ dentre todos os objetos conexos em $\mathcal{U}_{\boldsymbol{c}}\left(\mathcal{S}_{1}, \mathcal{S}_{0}\right)$, garantindo uma solução ótima de segmentação de acordo com o Teorema 2, cuja prova é apresentada no Apêndice C. 
Teorema 2 (Otimalidade do corte pela COIFT) Para dados dois conjuntos de sementes $\mathcal{S}_{\mathbf{1}}$ $e \mathcal{S}_{\mathbf{0}}$, qualquer objeto segmentado $\mathcal{O}_{c}$ calculado pelo algoritmo da COIFT (Algoritmo 2) maximiza $E(\mathcal{O})$ (Equação 3.16) dentre todos os resultados possiveis de segmentação em $\mathcal{U}_{c}\left(\mathcal{S}_{1}, \mathcal{S}_{0}\right)$. Isto é:

$$
E\left(\mathcal{O}_{c}\right)=\max _{\mathcal{O} \in \mathcal{U}_{c}\left(\mathcal{S}_{1}, \mathcal{S}_{0}\right)} E(\mathcal{O})
$$

\subsubsection{Algoritmo da COIFT}

Seja $\mathcal{E}: \mathcal{I} \rightarrow \mathbb{R}$ o mapa de energias contendo $E_{\{t\}}$ para todo vértice $t \in \mathcal{I}$ (isto é, $\mathcal{E}(t)=E_{\{t\}}$ ). O algoritmo da COIFT (Algoritmo 2, pág. 47) compreende essencialmente três passos:

- Passo 1: Calcular $E_{\{t\}}$ para todo $t \in \mathcal{I}$.

- Passo 2: Interconectar sementes não conexas em $\mathcal{S}_{1}$, calculando caminhos passando pelos vértices $t$ com energia máxima $E_{\{t\}}$, resultando num novo conjunto conexo de sementes internas $\mathcal{S}_{1}^{c}$. Para essa finalidade usamos a função $f_{\text {river }}^{\prime}$, a qual é um caso particular da função de custo $f_{\text {river }}$ (Equação 3.20), definida como

$$
\begin{aligned}
f_{\text {river }}^{\prime}(\langle t\rangle) & = \begin{cases}-1, & \text { se } t=s^{*} \\
+\infty, & \text { caso contrário }\end{cases} \\
f_{\text {river }}^{\prime}\left(\pi_{s} \cdot\langle s, t\rangle\right) & =\omega_{v}(t)
\end{aligned}
$$

tal que $\omega_{v}(t)=K_{1}-E_{\{t\}}, K_{1}=\max _{t \in \mathcal{I}} E_{\{t\}}$ e $s^{*}$ é um vértice arbitrário em $\mathcal{S}_{1}$.

- Passo 3: Executar o método OIFT (IFT com $f^{\sigma^{\top}}$ ) (Equação 3.19) a partir dos conjuntos de sementes $\mathcal{S}_{1}^{c}$ e $\mathcal{S}_{\mathbf{0}}$, gerando um resultado final conexo (Figura 4.6).

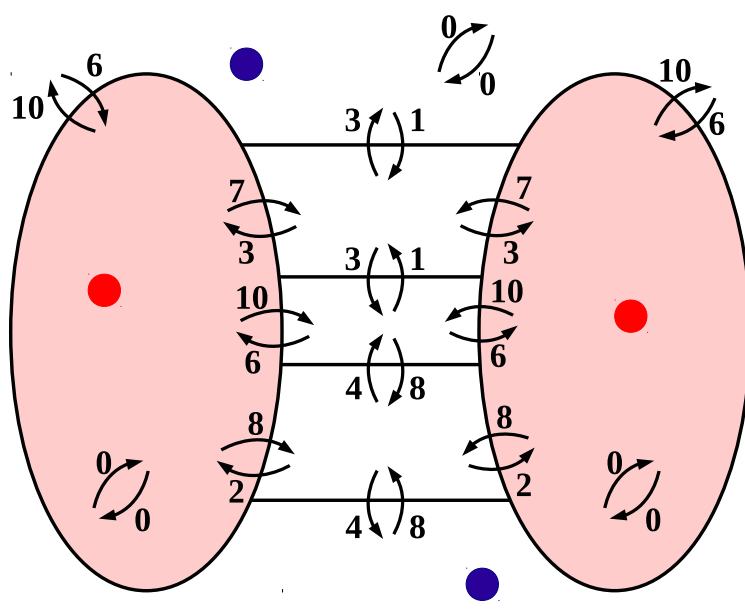

(a) $E(\mathcal{O})=7$

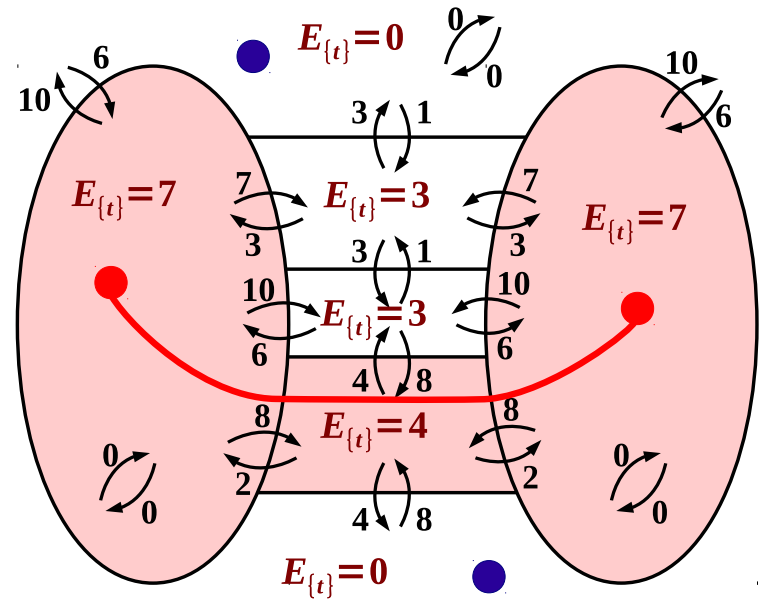

(b) $E(\mathcal{O})=4$

Figura 4.6: Representação gráfica com resultados das segmentações por: (a) OIFT. (b) COIFT. O caminho riverbed (linha vermelha) interconecta as sementes internas não conexas atravessando regiões com valores altos de $E_{\{t\}}$. 


\section{Algoritmo 2 - Algoritmo DA COIFT}

Entrada: $\quad$ Digrafo $G=\langle\mathcal{I}, \mathcal{A}, \omega\rangle$, conjunto de sementes $\mathcal{S}_{1}$ e $\mathcal{S}_{\mathbf{0}}$ e função de custo $f^{0^{\top}}$.

SAÍdA: $\quad$ Um componente $\langle L, P, V\rangle$ contendo o mapa de rótulos $L$, a floresta de espalhamento $P$ e o mapa de custos de caminho $V$.

Auxiliares: Mapa de custos mínimos de caminho $V_{\text {opt }}^{f_{\text {max }}^{\# S_{0}}}$, mapa de energias $\mathcal{E}: \mathcal{I} \rightarrow \mathbb{R}$ e conjunto de sementes $\mathcal{S}_{1}^{c}$.

1. Calcule $\left\langle-,-, V_{\text {opt }}^{f_{\max } S_{0}}\right\rangle \leftarrow \operatorname{IFT}\left(G, \varnothing, \mathcal{S}_{\mathbf{0}}, f_{\max }^{\# S_{0}}\right)$

2. Para cada $t \in \mathcal{I}$, faça

3. $\quad \mathrm{L} \quad \mathcal{E}(t) \leftarrow V_{\text {opt }}^{f_{\max }^{\# \mathcal{S}_{0}}}(t)$

4. Calcule $\mathcal{S}_{1}^{c} \leftarrow N e w \operatorname{ObjSeeds}\left(G, \mathcal{S}_{1}, \mathcal{E}\right)$

5. Calcule $\langle L, P, V\rangle \leftarrow \operatorname{IFT}\left(G, \mathcal{S}_{1}^{c}, \mathcal{S}_{0}, f^{\sigma^{\top}}\right)$

Retorne $\langle L, P, V\rangle$

\section{Algoritmo 3 - Algoritmo NewObjSeeds}

Entrada: Digrafo $G=\langle\mathcal{I}, \mathcal{A}, \omega\rangle$, conjunto de sementes $\mathcal{S}_{1}$ e mapa de energias $\mathcal{E}: \mathcal{I} \rightarrow \mathbb{R}$.

SAÍdA: $\quad$ Conjunto de sementes $\mathcal{S}_{1}^{c}$.

Auxiliares: Floresta de caminhos $P_{\text {river }}$, função de custo $f_{\text {river }}^{\prime}$ e variáveis $s^{*}, K_{1}$ e pred.

1. $s^{*} \leftarrow \arg \min _{t \in \mathcal{S}_{1}} \mathcal{E}(t)$

2. $\quad K_{1} \leftarrow \max _{t \in \mathcal{I}} \mathcal{E}(t)$

3. Para cada $t \in \mathcal{I}$, faça

4. $\quad \mathrm{L} \quad \omega_{v}(t) \leftarrow K_{1}-\mathcal{E}(t)$.

5. Calcule $\left\langle-, P_{\text {river }},-\right\rangle \leftarrow \operatorname{IFT}\left(G,\left\{s^{*}\right\}, \varnothing, f_{\text {river }}^{\prime}\right)$

6. $\mathcal{S}_{1}^{c} \leftarrow \varnothing$

7. Para cada $t \in \mathcal{S}_{1}$, faça

8. $\quad$ pred $\leftarrow t$

9. $\quad$ Enquanto pred $\neq$ nil, faça

10. $\quad \mid \mathcal{S}_{1}^{c} \leftarrow \mathcal{S}_{1}^{c} \cup\{$ pred $\}$

11. $\left[\quad\left[\right.\right.$ pred $\leftarrow P_{\text {river }}($ pred $)$

Retorne $\mathcal{S}_{1}^{c}$

\subsection{Segmentação de um objeto $\gamma$-conexo via COIFT}

Nesta seção mostramos como a COIFT pode ser estendida para segmentar objetos $\gamma$-conexos em relação a $\mathcal{S}_{1}$ (Definição 18, pág. 43), tornando-a mais ajustável para diferentes objetos (Figura 4.7). Nessa configuração a COIFT é chamada de COIFT com largura ajustável. 


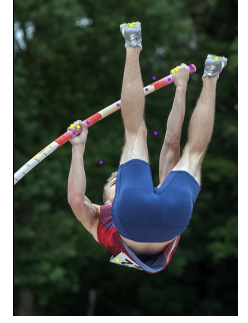

(a1)

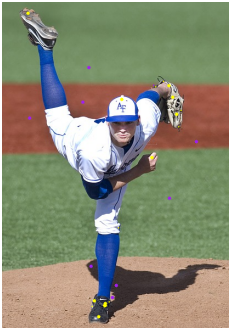

(b1)

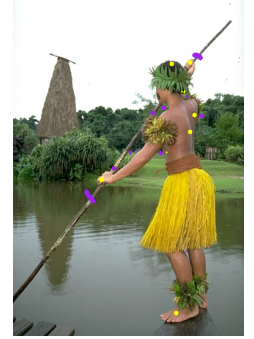

$(\mathrm{c} 1)$

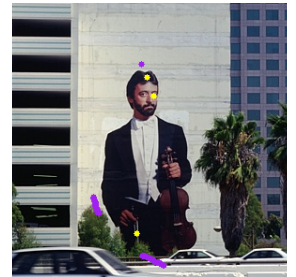

$(\mathrm{d} 1)$

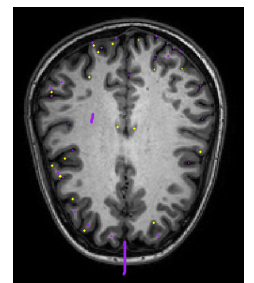

(e1)

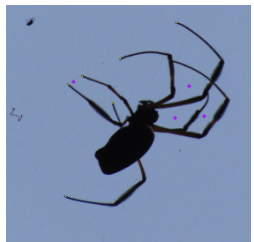

(f1)

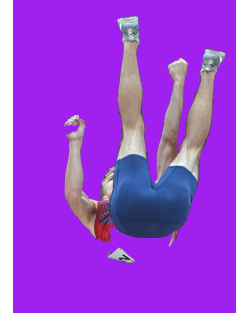

$(\mathrm{a} 2)$

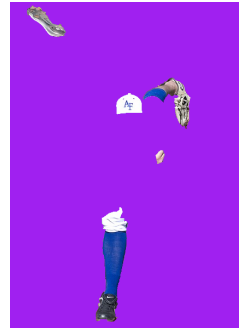

(b2)

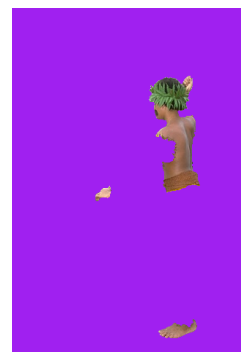

$(\mathrm{c} 2)$

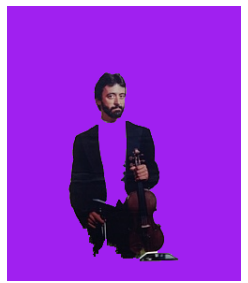

(d2)

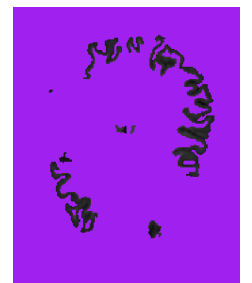

(e2)

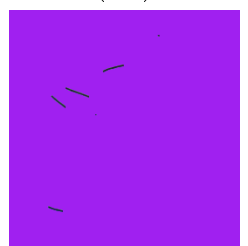

(f2)

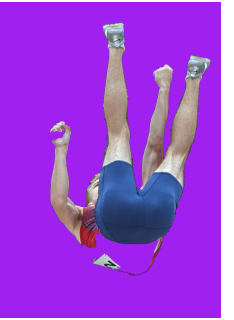

(a3)

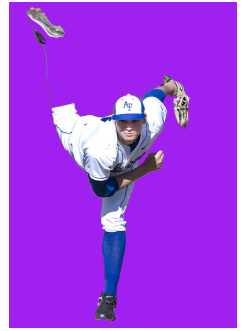

(b3)

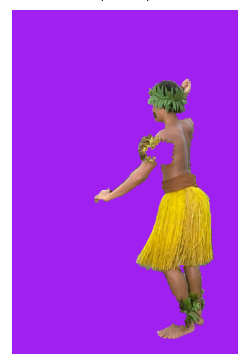

(c3)

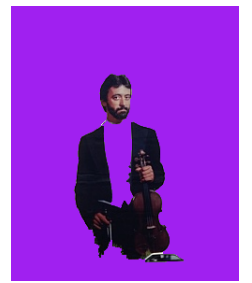

(d3)

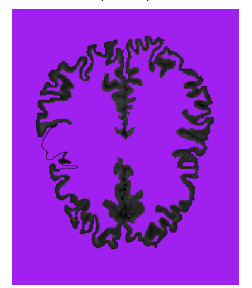

(e3)

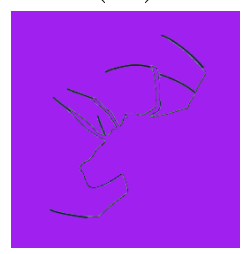

(f3)

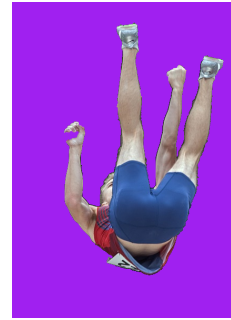

(a4)

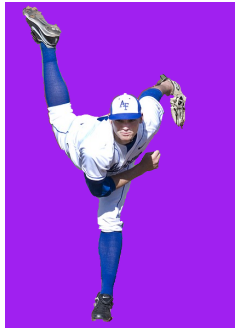

(b4)

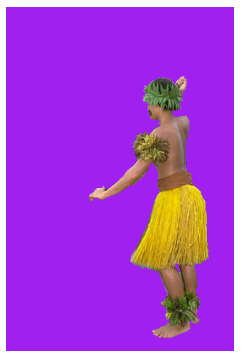

(c4)

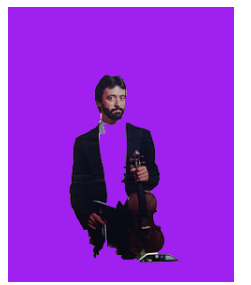

(d4)

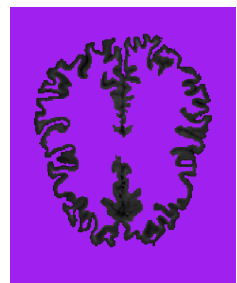

(e4)

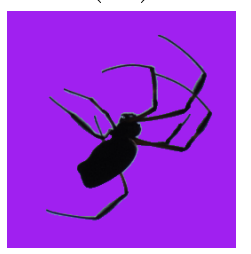

(f4)

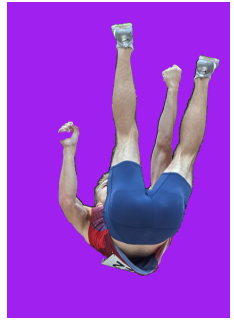

(a5)

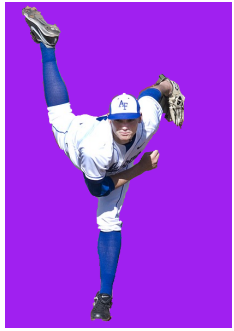

(b5)

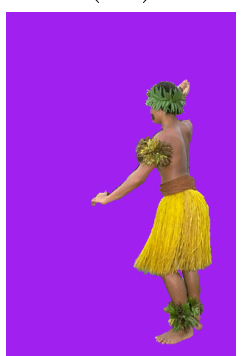

(c5)

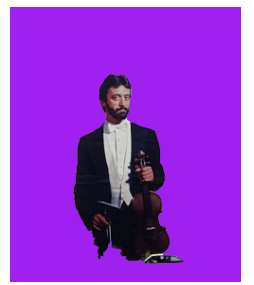

(d5)

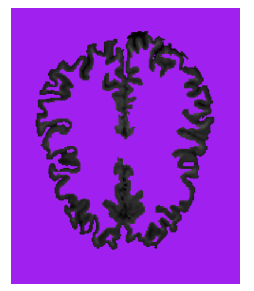

(e5)

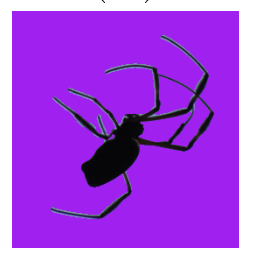

(f5)

Figura 4.7: Exemplos de segmentações via COIFT com largura ajustável. A primeira coluna representa as imagens de entrada com marcadores selecionados pelo usuário. A segunda coluna, segmentações sem restrição de conexidade (OIFT com $\alpha=0.0)$. A terceira coluna, segmentações com COIFT $(\alpha=0.0$ e $\gamma=0.0)$. A quarta coluna, segmentações com COIFT $(\alpha=0.0$ e $\gamma=1.0)$. A quinta coluna, segmentações com COIFT $(\alpha=0.0 e$ $\gamma=2.0)$. Os resultados propostos pela COIFT garantem objetos $\gamma$-conexos em relação as sementes internas. 
Seja $E_{\mathcal{V}_{\odot}(t, \gamma)}$ a energia ótima a partir de um único disco $\mathcal{V}_{\odot}(t, \gamma)$ (Definição 17, pág. 41), usando $\mathcal{V}_{\odot}(\boldsymbol{t}, \gamma)$ como sementes internas. A partir das Proposições 2 e 3 (págs. 44 e 45 ) temos que

$$
E_{\mathcal{V}_{\odot}(t, \gamma)}=\min _{p \in \mathcal{V}_{\odot}(t, \gamma)} E_{\{p\}}
$$

e

$$
E_{\mathcal{V}_{\odot}(t, \gamma)}=\min _{p \in \mathcal{V}_{\odot}(t, \gamma)} V_{o p t}^{f_{\max }^{H \mathcal{S}_{0}}}(p)
$$

Sejam $\mathcal{U}_{\boldsymbol{c}}^{\gamma}\left(\mathcal{S}_{1}, \mathcal{S}_{\mathbf{0}}\right)$ o conjunto de todos os objetos $\gamma$-conexos em relação a $\mathcal{S}_{\mathbf{1}}$ satisfazendo as restrições de sementes (isto é, $\mathcal{U}_{\boldsymbol{c}}^{\gamma}\left(\mathcal{S}_{1}, \mathcal{S}_{0}\right) \subseteq \mathcal{U}\left(\mathcal{S}_{1}, \mathcal{S}_{0}\right)$ ), definido por:

$$
\mathcal{U}_{c}^{\gamma}\left(\mathcal{S}_{1}, \mathcal{S}_{0}\right)=\left\{\mathcal{O} \in \mathcal{U}\left(\mathcal{S}_{1}, \mathcal{S}_{0}\right) \mid \mathcal{O} \text { é } \gamma \text {-conexo em relação a } \mathcal{S}_{1}\right\}
$$

Seja $\boldsymbol{R}^{\gamma}(\mathcal{O})=\bigcup_{k} C_{k}$, a partição de $\boldsymbol{R}^{\gamma}(\mathcal{O})$ em seus componentes fortemente conexos $C_{k}$, $k=1, \ldots, n_{c}$.

A partir da definição do objeto $\gamma$-conexo (Definição 18, pág. 43), podemos concluir que se $\mathcal{O}$ é $\gamma$-conexo então para quaisquer $s_{i}, s_{j} \in \mathcal{S}_{1}$, temos que:

1. $\mathcal{V}_{\odot}\left(s_{i}, \gamma\right) \subseteq \mathcal{O}$

2. $\left\{s_{i}, s_{j}\right\} \subseteq C_{k}$

Assim, da Proposição 2 (pág. 44) podemos deduzir que um objeto ótimo $\mathcal{O}_{\text {opt }} \in \mathcal{U}_{\boldsymbol{c}}^{\gamma}\left(\mathcal{S}_{1}, \mathcal{S}_{\mathbf{0}}\right)$ possui uma energia máxima definida por

$$
E\left(\mathcal{O}_{\text {opt }}\right) \leqslant \min _{t \in \mathcal{S}_{1} \subseteq C_{k}} E_{\mathcal{V}_{\odot}(t, \gamma)}
$$

A COIFT com largura ajustável maximiza a energia $E(\mathcal{O})$ (Equação 3.16) em um digrafo simétrico fortemente conexo $G$ dentre todos os objetos $\gamma$-conexos em $\mathcal{U}_{\boldsymbol{c}}^{\gamma}\left(\mathcal{S}_{1}, \mathcal{S}_{\mathbf{0}}\right)$, garantindo uma solução ótima de segmentação de acordo com o Teorema 3, cuja prova é apresentada no Apêndice D.

Teorema 3 (Otimalidade do corte pela COIFT com largura ajustável) Para dados dois conjuntos de sementes $\mathcal{S}_{\mathbf{1}}$ e $\mathcal{S}_{\mathbf{0}}$, qualquer objeto segmentado $\mathcal{O}_{\boldsymbol{c}}^{\gamma}$ calculado pelo algoritmo da COIFT com largura ajustável (Algoritmo 4) maximiza $E(\mathcal{O})$ (Equação 3.16) dentre todos os resultados possíveis de segmentação em $\mathcal{U}_{c}^{\gamma}\left(\mathcal{S}_{1}, \mathcal{S}_{0}\right)$. Isto é:

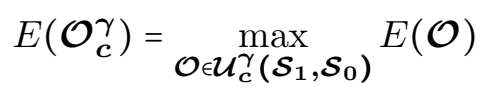

\subsubsection{Algoritmo da COIFT com largura ajustável}

Seja $\mathcal{E}_{\odot}: \mathcal{I} \rightarrow \mathbb{R}$ o mapa de energias contendo $E_{\mathcal{V}_{\odot}(t, \gamma)}$ para todo vértice $t \in \mathcal{I}$, isto é, $\mathcal{E}_{\odot}(t)=E_{\mathcal{V}_{\odot}(t, \gamma)}$. O algoritmo da COIFT com largura ajustável (Algoritmo 4, pág. 50) compreende essencialmente três passos:

- Passo 1: Calcular $E_{\mathcal{V}_{\odot}(t, \gamma)}$ para todo $t \in \mathcal{I}$ (Figuras $4.8 \mathrm{c}, \mathrm{d}$ ). 
- Passo 2: Interconectar sementes em $\mathcal{S}_{1}$, calculando caminhos passando pelos vértices $t$ com máxima energia $E_{\mathcal{V}_{\odot}(t, \gamma)}$, resultando em um novo conjunto conexo de sementes internas $\mathcal{S}_{1}^{c}$ (Figura 4.8e). Para essa finalidade usamos a função de custo $f^{\prime}{ }_{\text {river }}$ (Equação 4.13), tal que $\omega_{v}(t)=K_{2}-E_{\mathcal{V}_{\odot}(\boldsymbol{t}, \boldsymbol{\gamma})}$ e $K_{2}=\max _{t \in \mathcal{I}} E_{\mathcal{V}_{\odot}(\boldsymbol{t}, \boldsymbol{\gamma})}$. A fim de garantir a largura do objeto $\gamma$-conexo em relação ao conjunto $\mathcal{S}_{1}$, consideramos os discos centralizados no conjunto $\mathcal{S}_{1}^{c}$ para obter um novo conjunto conexo de sementes internas $\mathcal{S}_{1}^{\gamma c}$.

- Passo 3: Executar o método OIFT (IFT com $f^{0^{7}}$ ) (Equação 3.19) a partir dos conjuntos de sementes $\mathcal{S}_{\mathbf{1}}^{\gamma \boldsymbol{c}}$ e $\mathcal{S}_{\mathbf{0}}$, gerando um resultado final $\gamma$-conexo (Figura $4.8 \mathrm{f}$ ).

\section{Algoritmo 4 - Algoritmo da COIFT com largura Ajustável}

Entrada: $\quad$ Digrafo $G=\langle\mathcal{I}, \mathcal{A}, \omega\rangle$, conjunto de sementes $\mathcal{S}_{1}$ e $\mathcal{S}_{0}$ e função de custo $f^{0^{7}}$.

SAída: $\quad$ Um componente $\langle L, P, V\rangle$ contendo o mapa de rótulos $L$, a floresta de espalhamento $P$ e o mapa de custos de caminho $V$.

Auxiliares: Mapa de custos mínimos de caminho $V_{\text {opt }}^{f_{\text {max }}^{\# S_{0}}}$, mapa de energias $\mathcal{E}_{\odot}: \mathcal{I} \rightarrow \mathbb{R}$ e conjunto de sementes $\mathcal{S}_{1}^{\gamma c}$.

1. Calcule $\left\langle-,-, V_{\text {opt }}^{f_{\text {max }}^{H S_{0}}}\right\rangle \leftarrow \operatorname{IFT}\left(G, \varnothing, \mathcal{S}_{0}, f_{\max }^{\# S_{0}}\right)$

2. Para cada $t \in \mathcal{I}$, faça

3. $\quad \mathrm{L} \quad \mathcal{E}_{\odot}(t) \leftarrow \min _{p \in \mathcal{V}_{\odot}(t, \gamma)} V_{\text {opt }}^{f_{\text {max }}^{\sharp \mathcal{S}_{0}}}(p)$

4. Calcule $\mathcal{S}_{1}^{\gamma c} \leftarrow N e w D i s k \operatorname{Obj} \operatorname{Seeds}\left(G, \mathcal{S}_{1}, \mathcal{E}_{\odot}\right)$

5. Calcule $\langle L, P, V\rangle \leftarrow \operatorname{IFT}\left(G, \mathcal{S}_{1}^{\gamma c}, \mathcal{S}_{\mathbf{0}}, f^{\sigma^{\gamma}}\right)$

Retorne $\langle L, P, V\rangle$

\section{Algoritmo 5 - Algoritmo NewDiskObjSeeds}

Entrada: $\quad$ Digrafo $G=\langle\mathcal{I}, \mathcal{A}, \omega\rangle$, conjunto de sementes $\mathcal{S}_{1}$ e mapa de energias $\mathcal{E}_{\odot}: \mathcal{I} \rightarrow \mathbb{R}$.

SAÍdA: $\quad$ Conjunto de sementes $\mathcal{S}_{1}^{\gamma c}$.

Auxiliares: Floresta de caminhos $P_{\text {river }}$, função de custo $f_{\text {river }}^{\prime}$, conjunto de sementes $\mathcal{S}_{1}^{c}$ e variáveis $s^{*}, K_{2}$ e pred.

1. $s^{*} \leftarrow \arg \min _{t \in \mathcal{S}_{1}} \mathcal{E}_{\odot}(t)$

2. $K_{2} \leftarrow \max _{t \in \mathcal{I}} \mathcal{E}_{\odot}(t)$

3. Para cada $t \in \mathcal{I}$, faça

4. $\quad \mathrm{L} \quad \omega_{v}(t) \leftarrow K_{2}-\mathcal{E}_{\odot}(t)$.

5. Calcule $\left\langle-, P_{\text {river }},-\right\rangle \leftarrow \operatorname{IFT}\left(G,\left\{s^{*}\right\}, \varnothing, f_{\text {river }}^{\prime}\right)$

6. $\mathcal{S}_{1}^{c} \leftarrow \varnothing$

7. Para cada $t \in \mathcal{S}_{1}$, faça

8. $\quad$ pred $\leftarrow t$

9. $\quad$ Enquanto pred $\neq$ nil, faça

10.

11.

$$
\mathcal{S}_{1}^{c} \leftarrow \mathcal{S}_{1}^{c} \cup\{\text { pred }\}
$$

12. $\mathcal{S}_{1}^{\gamma c} \leftarrow \varnothing$

13. Para cada $t \in \mathcal{S}_{1}^{c}$, faça

14. $\mathrm{L} \quad \mathcal{S}_{1}^{\gamma c} \leftarrow \mathcal{S}_{1}^{\gamma c} \cup \mathcal{V}_{\odot}(t, \gamma)$

Retorne $\mathcal{S}_{1}^{\gamma c}$

A principal diferença em relação ao algoritmo original da COIFT (Algoritmo 2, pág. 50) está nos passos 1 e 2 , nos quais temos que calcular $E_{\mathcal{V}_{\odot}(t, \gamma)}$ em vez de $E_{\{t\}}$. 


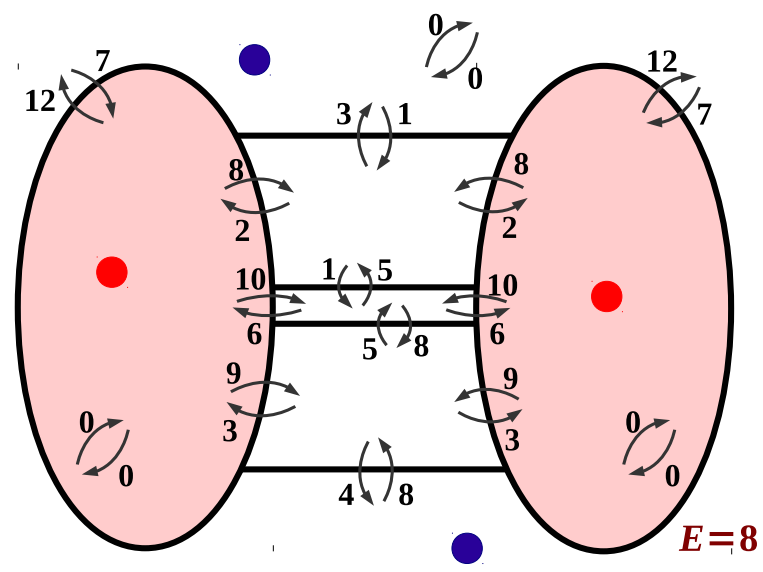

(a)

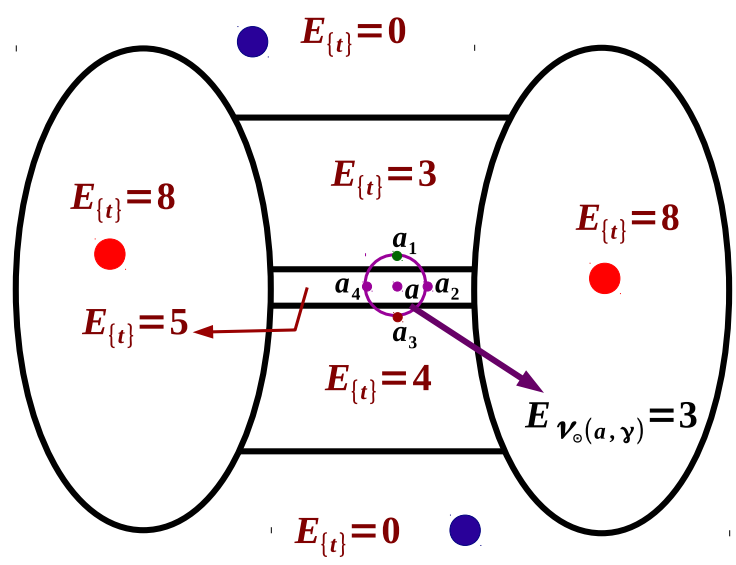

(c)

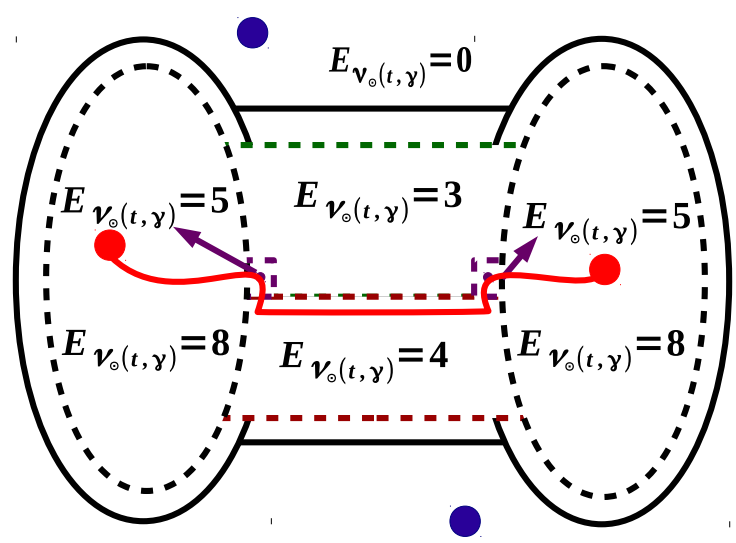

(e)

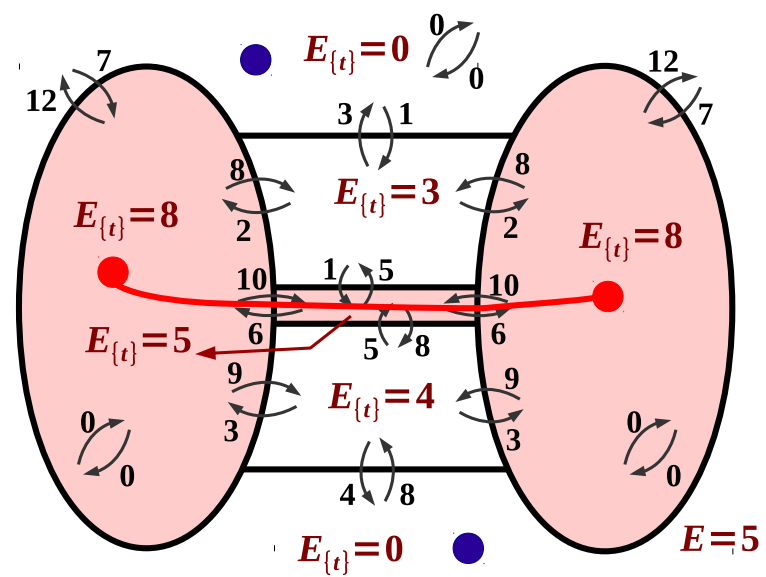

(b)

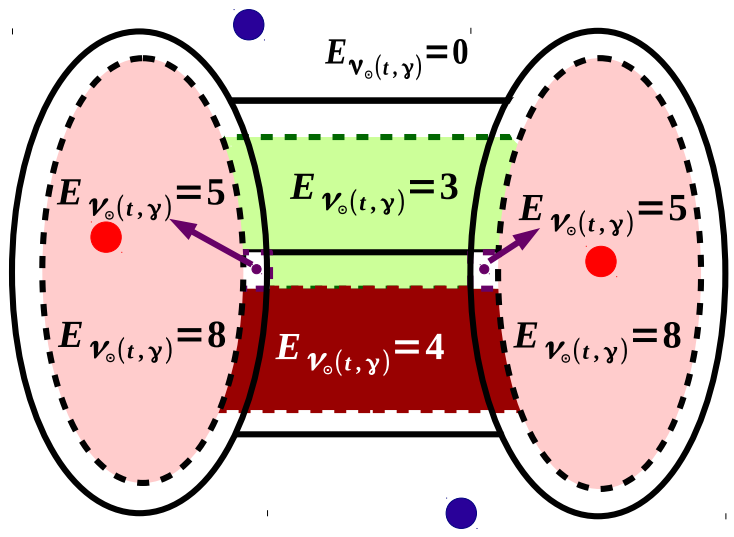

(d)

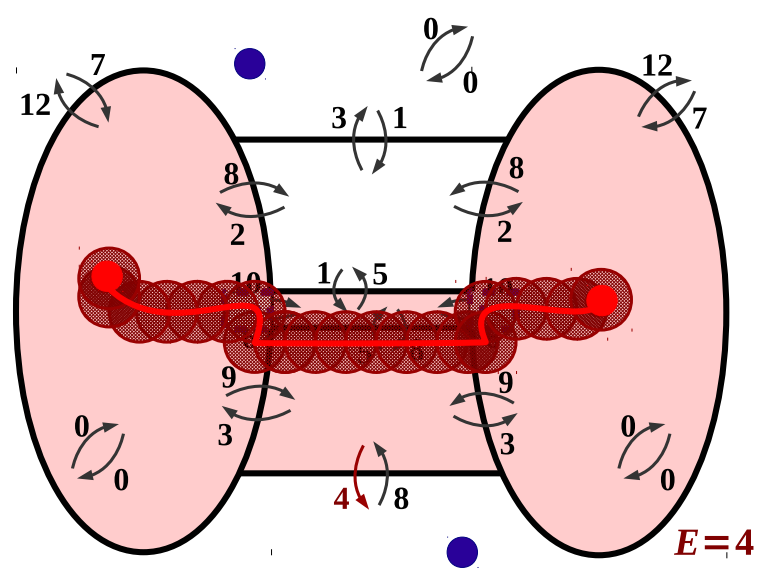

(f)

Figura 4.8: Representação gráfica com resultados de segmentações dadas por: (a) OIFT. (b) COIFT ( $\gamma=0)$, o caminho riverbed interconectando as sementes e os valores de $E_{\{t\}}$ para cada região são mostrados em vermelho. (c-f) COIFT com $\gamma=1.0$. Em (c) podemos observar como a energia do disco é calculada para o pixel $a$, isto é, $E_{\mathcal{V}_{\odot}(a, \gamma)}=\min \left\{E_{\left\{a_{1}\right\}}, E_{\left\{a_{2}\right\}}, E_{\left\{a_{3}\right\}}, E_{\left\{a_{4}\right\}}, E_{\{a\}}\right\}=\min \{3,5,4,5,5\}=3$. As regiões coloridas em (d) representam as regiões de energia final $E_{\mathcal{V}_{\odot}(t, \gamma)}$ para todo $t \in \mathcal{I}$. (e) $O$ caminho riverbed interconectando as sementes internas é mostrado. (f) Um objeto 1 - conexo em relação ao conjunto de sementes internas iniciais é obtido. 
Na prática, $E_{\boldsymbol{V}_{\odot}(t, \gamma)}$ pode ser calculada pela erosão do mapa de custos $V_{o p t}^{f_{\max }^{\# \mathcal{S}_{0}}}$ com raio de erosão $\gamma$

Na Figura 4.9 podemos observar um exemplo de segmentação de um pássaro com a OIFT e COIFT com $\gamma=1.0$, na qual é mostrada a vantagem do uso de restrições de $\gamma$-conexidade para melhorar o resultado da segmentação.

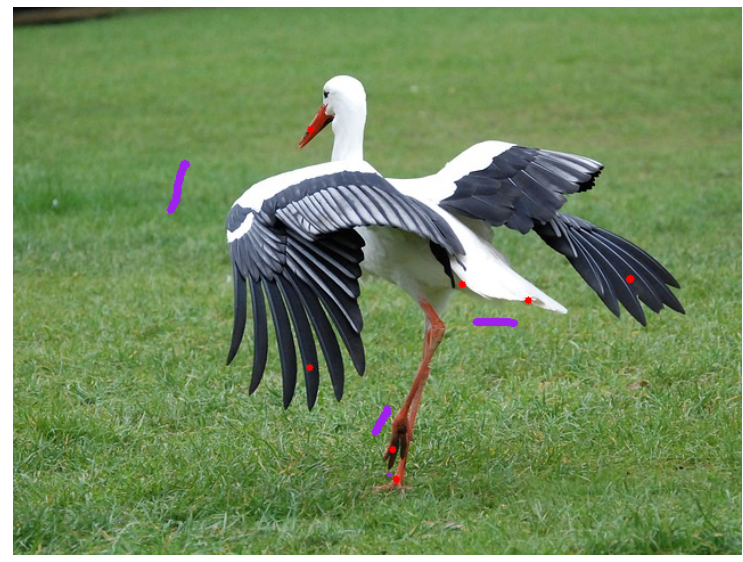

(a)

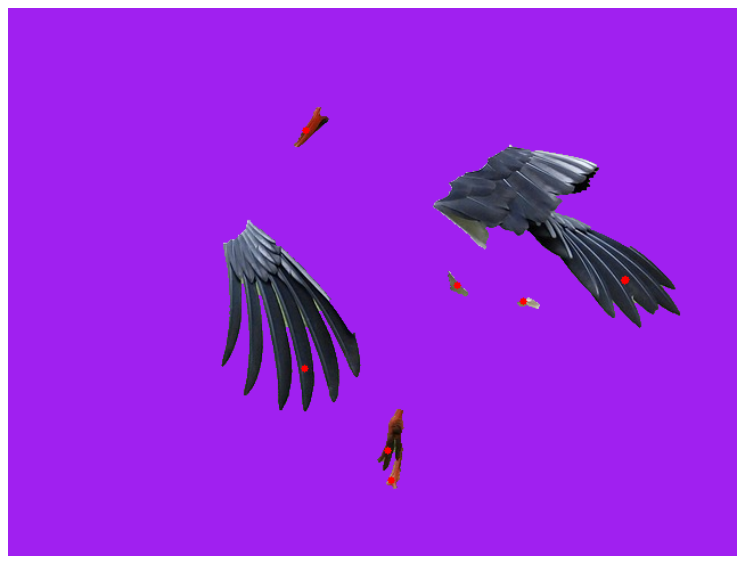

(b)

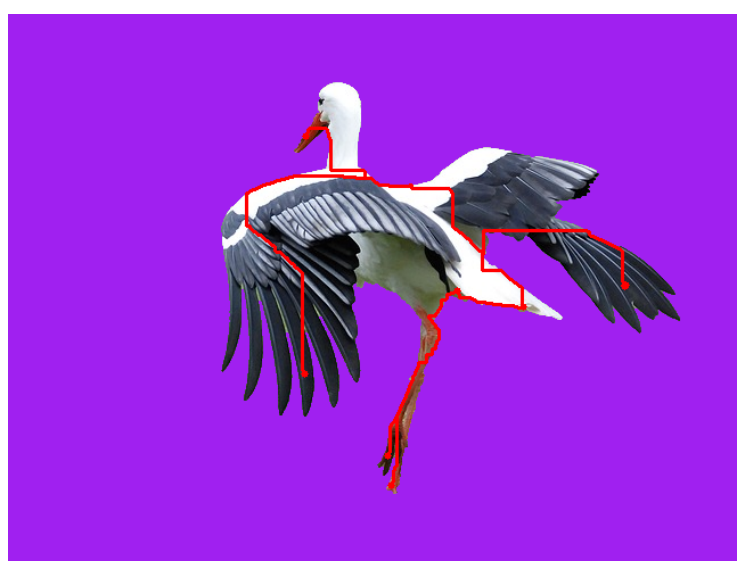

(c)

Figura 4.9: Exemplo de segmentação: (a) Imagem com marcadores escolhidos pelo usuário. (b) Segmentação com OIFT. (c) Segmentação com COIFT $(\gamma=1.0)$. As sementes internas e o caminho riverbed interconectando as mesmas são mostrados em vermelho. 


\subsubsection{Tratamento de empates de energia}

Embora os resultados da COIFT melhorem os resultados da segmentação incorporando as restrições de conexidade na sua definição, conforme apresentado nas seções anteriores, ela não lida com os possíveis empates de energia que podem ocorrer durante a relação dos caminhos de riverbed interconectando as sementes internas.

As alterações propostas a seguir não afetam os resultados teóricos apresentados nas seções anteriores, mas melhoram a qualidade da segmentação resultante.

\section{Tratamento de empate nas regiões de energia $E_{\mathcal{V}_{\odot}(t, \gamma)}$}

Um exemplo de empate nas regiões de energia $E_{\mathcal{V}_{\odot}(\boldsymbol{t}, \boldsymbol{\gamma})}$ pode ser observado na Figura $4.8 \mathrm{e}$, no qual um caminho riverbed alternativo poderia atravessar regiões afastadas das bordas no mapa de energias $E_{\{t\}}$ (Figura 4.8c). A seleção de caminhos afastados das fronteiras dos componentes de mesma energia ajuda a evitar vazamentos em regiões de alto contraste coladas ao objeto, tais como sombras.

Assim, a fim de obter caminhos mais centralizados, nós modificamos a função $f_{\text {river }}^{\prime}$ (Equação 4.13) para favorecer a seleção de caminhos de conexão afastados das bordas das regiões de energia $E_{\{t\}}$ considerando um outro parâmetro no peso associado ao vértice $t$, tornando $f_{\text {river }}^{\prime}$ em uma função lexicográfica $f_{\text {river }}^{\prime \prime}$.

$$
\begin{aligned}
f_{\text {river }}^{\prime \prime}(\langle t\rangle) & = \begin{cases}-1, & \text { se } t=s^{*} \\
+\infty, & \text { caso contrário }\end{cases} \\
f_{\text {river }}^{\prime \prime}\left(\pi_{s} \cdot\langle s, t\rangle\right) & =\vec{v}(t)
\end{aligned}
$$

tal que $\vec{v}(t)=\left(K_{2}-E_{\mathcal{V}_{\odot}(t, \gamma)}, K_{3}-E_{\mathcal{V}_{\odot}(t, 2 \gamma)}\right), K_{2}=\max _{t \in \mathcal{I}} E_{\mathcal{V}_{\odot}(t, \gamma)}, K_{3}=\max _{t \in \mathcal{I}} E_{\mathcal{V}_{\odot}(t, 2 \gamma)}$ e $s^{*}$ é um vértice arbitrário em $\mathcal{S}_{1}$.

\section{Tratamento de consistência com resultado de OIFT}

Sempre que a segmentação pela OIFT já resulta em um único componente conexo, poderíamos esperar que a COIFT produziria o mesmo resultado. Porém, em casos onde existem soluções múltiplas com mesmo valor de energia ótima, a COIFT pode selecionar um caminho de conexão alternativo pelo riverbed, levando a uma segmentação diferente, como ilustrado na Figura 4.10. Com o fim de garantir o mesmo resultado nesses casos, mudamos a função $f_{\text {river }}^{\prime \prime}$ (Equação 4.19) para favorecer a seleção de caminhos de riverbed que passam pela segmentação da OIFT, atribuindo custos baixos para pixels no resultado erodido da OIFT (Figura 4.10f) com um raio $\gamma$. Note que, o resultado da OIFT pode ser usado como uma informação a priori da COIFT uma vez que um objeto segmentado pelo algoritmo da OIFT está incluso num objeto segmentado pelo algoritmo da COIFT (isto é, $\mathcal{O}^{\boldsymbol{O I F T}} \subseteq \mathcal{O}^{\boldsymbol{C O I F T}}$ ). Assim, consideramos a erosão da imagem de rótulos da OIFT (isto é, $\left.\hat{L}_{O I F T}\right)$, e modificamos a função $f_{\text {river }}^{\prime \prime}$ tal que um novo parâmetro no peso associado a todo vértice $t \in \mathcal{I}$ é considerado. Isto é, $f_{\text {river }}^{\prime \prime} \operatorname{com} \vec{v}(t)=\left(1-L_{O I F T}^{\gamma}(t), K_{2}-E_{\mathcal{V}_{\odot}(\boldsymbol{t}, \boldsymbol{\gamma})}, K_{3}-E_{\mathcal{V}_{\odot}(t, 2 \gamma)}\right)$, tal que $K_{2}=\max _{t \in \mathcal{I}} E_{\mathcal{V}_{\odot}(t, \gamma)}, K_{3}=\max _{t \in \mathcal{I}} E_{\mathcal{V}_{\odot}(t, 2 \gamma)}$ e $L_{O I F T}^{\gamma}$ representa o mapa resultante da erosão do resultado da segmentação pela OIFT com raio $\gamma$. 


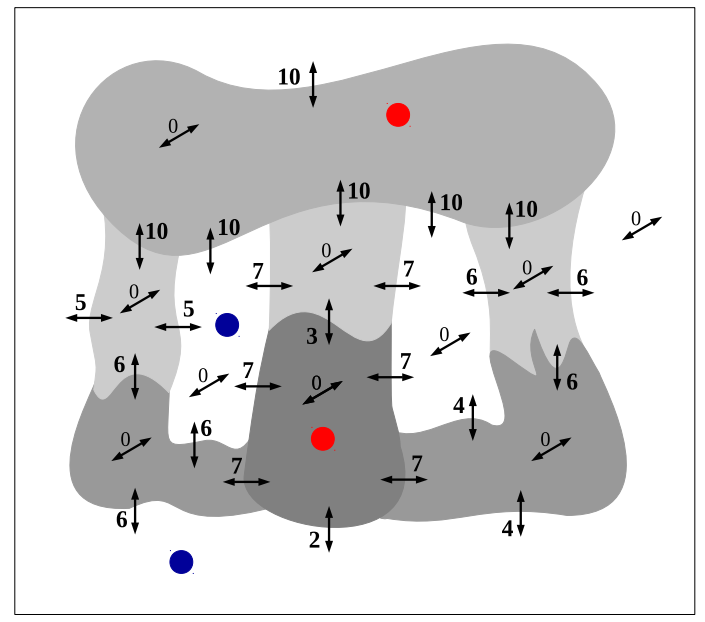

(a)

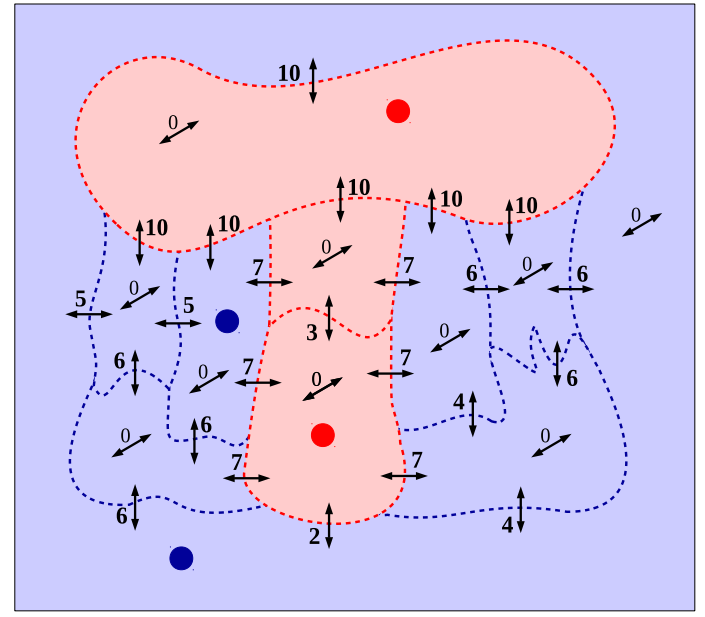

(b) $E(\mathcal{O})=2$

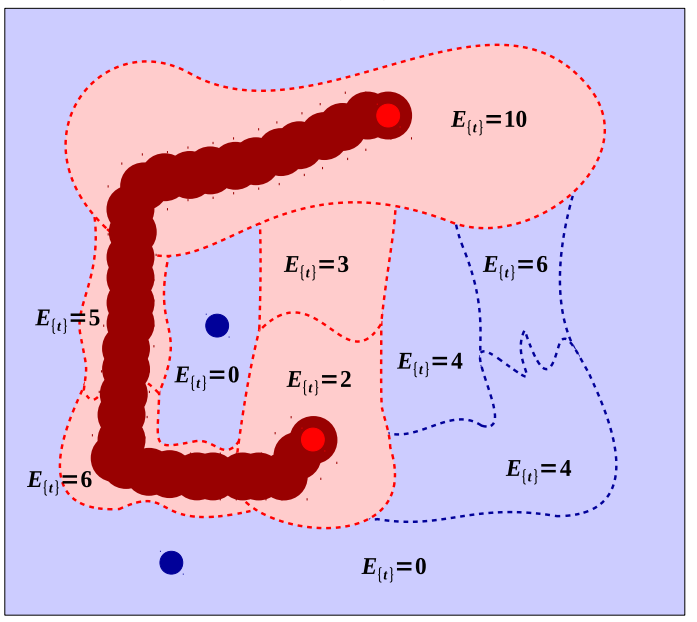

(c) $E(\mathcal{O})=2$

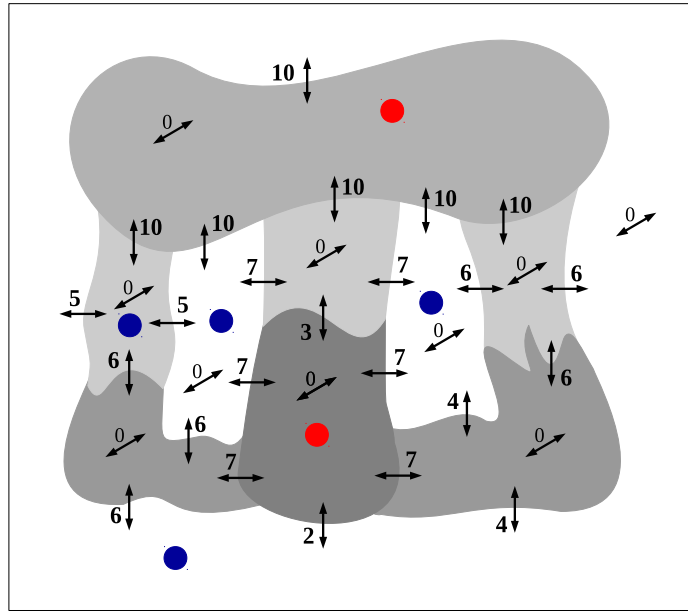

(d)

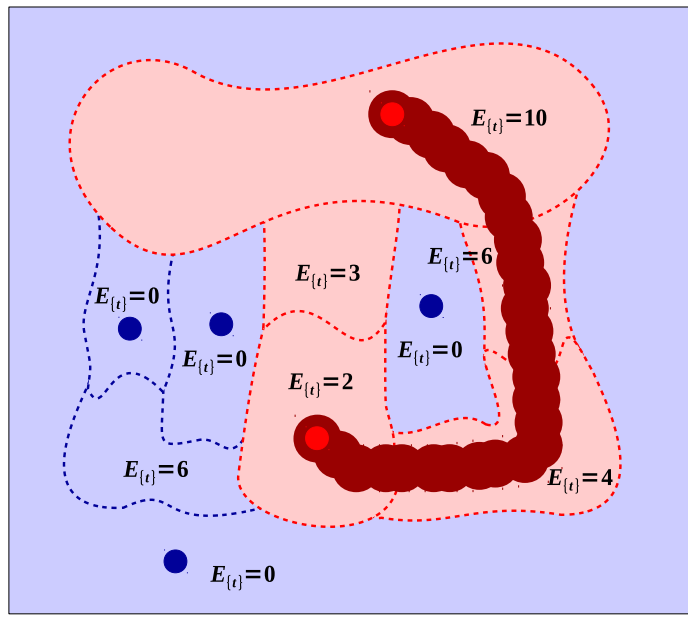

(e) $E(\mathcal{O})=2$

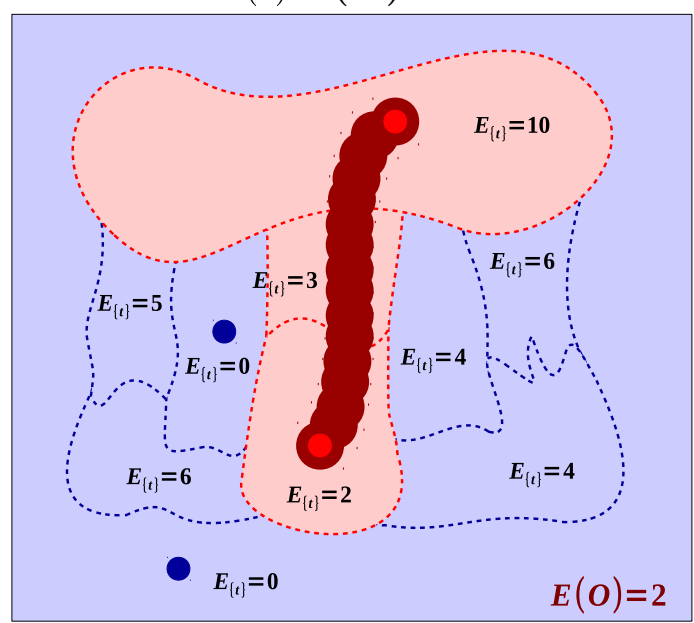

(f) $E(\mathcal{O})=2$

Figura 4.10: Representação esquemática da segmentação de uma imagem sintética pela OIFT e COIFT no caso de empates de energias. (a) Uma imagem sintética com sementes internas (marcadores vermelhos) e externas (marcadores azuis). (b) Resultado da OIFT. (c) Resultado da COIFT. Os valores de $E_{\{t\}}$ para cada região são mostrados. (d) Sementes externas adicionais são selecionadas. (e) Resultado atualizado pela COIFT. Notar que mesmo com mais sementes externas, a COIFT ainda não da a mesma solução da OIFT, a qual tem uma única componente conexa. (f) Resultado da COIFT considerando a função riverbed modificada que favorece pixels na OIFT. Notar que os resultados em (c), (e) e (f) tem a mesma energia (isto é, E = 2). Para resultados da COIFT, o caminho riverbed interconectando as sementes internas é mostrado em vermelho. 
Finalmente, a fim de obter caminhos de menor comprimento, podemos incorporar um quarto parâmetro na função de custo lexicográfica $f_{\text {river }}^{\prime \prime}$ (Equação 4.19), na qual o peso associado a todo vértice $t \in \mathcal{I}$ é

$$
\vec{v}(t)=\left(1-L_{O I F T}^{\gamma}(t), K_{2}-E_{\mathcal{V}_{\odot}(t, \gamma)}, K_{3}-E_{\mathcal{V}_{\odot}(t, 2 \gamma)}, f_{l e n}\left(\pi_{t}\right)\right)
$$

tal que $f_{l e n}\left(\pi_{t}\right)$ é uma função de custo que calcula o comprimento do caminho até o vértice $t$, definida por

$$
\begin{aligned}
f_{\text {len }}(\langle t\rangle) & = \begin{cases}0, & \text { se } t \in \mathcal{S}_{1} \cup \mathcal{S}_{0} \\
+\infty, & \text { caso contrário }\end{cases} \\
f_{\text {len }}\left(\pi_{s} \cdot\langle s, t\rangle\right) & =f_{\text {len }}\left(\pi_{s}\right)+1 .
\end{aligned}
$$

Na Figura 4.11 é mostrado um exemplo da segmentação de um inseto com a OIFT e a COIFT com $\gamma=1.0$ e $\gamma=4.0$, considerando o tratamento de empates de energia, sendo possível observar caminhos riverbed centralizados de menor comprimento (Figura 4.11c). Nessa figura, note que quando $\gamma=4.0$, o objeto alvo representa um objeto não 4-conexo em relação ao conjunto de sementes internas (marcadores vermelhos na Figura 4.11a). Nesse caso, a COIFT gera um novo conjunto de sementes internas $\mathcal{S}_{1}^{\gamma c}$ que passa por pixels pertencentes ao fundo, uma vez que a largura considerada dos discos, que é usada para detectar a violação de $\gamma$-conexidade, é maior do que a largura das partes finas do objeto (Figura 4.11d).

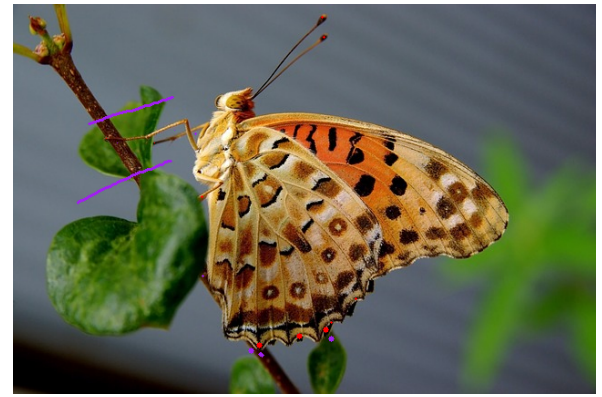

(a)

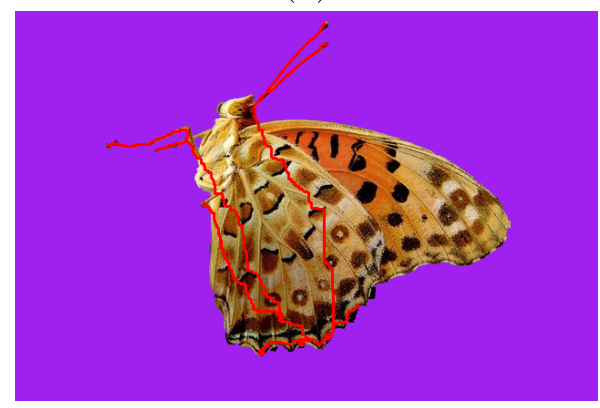

(c)

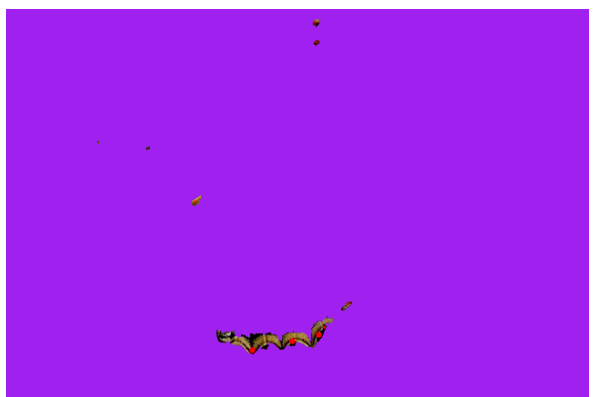

(b)

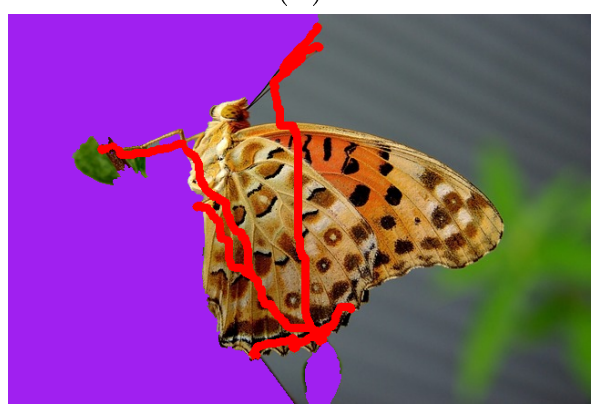

(d)

Figura 4.11: Exemplo de segmentação pela COIFT com largura ajustável considerando o tratamento de empates de energias. (a) Imagem com marcadores escolhidos pelo usuário. (b) Segmentação com OIFT. Em (c) e (d) são mostrados os resultados da segmentação pela COIFT com $\gamma=1.0$ e $\gamma=4.0$, respectivamente. Note que, para valores maiores de $\gamma$ a restrição de $\gamma$-conexidade é mais forte, sendo o objeto alvo a ser segmentado um objeto não 4-conexo em relação ao conjunto de sementes internas (marcadores vermelhos em (a)). 


\subsection{Resultados experimentais}

Nesta seção detalhamos cinco experimentos realizados para avaliar o desempenho da COIFT, sendo que para cada experimento foi usado um conjunto de imagens diferente. No primeiro experimento avaliamos a COIFT sem largura ajustável, no segundo e terceiro experimento avaliamos a COIFT com largura ajustável sem considerar o tratamento de empates de energia, e finalmente no quarto e quinto experimento avaliamos a COIFT com largura ajustável considerando o tratamento de empates de energia.

A seguir apresentamos os conjuntos de imagens usados nos nossos experimentos, as abordagens usadas para o cálculo automático das sementes, os métodos comparados, bem como as curvas de desempenho obtidas.

\subsubsection{Conjuntos de imagens}

Objetos com partes finas e alongadas são difíceis a serem segmentados por métodos sem restrição de conexidade, comumente gerando quebra de conexidade em suas partes finas e, consequentemente, requerendo um maior esforço por parte do usuário. Note que a seleção de mais marcadores nestas partes finas precisa ser feita de modo cauteloso para não invadir o fundo, com tamanho de pincel pequeno e visualização com zoom. Assim, a fim de demonstrar a vantagem do uso das restrições de conexidade, os cinco conjuntos de imagens usados nos nossos experimentos, descritos a seguir, possuem objetos com essas características.

- O primeiro conjunto está composto por 40 imagens de Tomografia Computadorizada (TC), com gabarito conhecido, correspondente a estudos da coluna cervical de 10 pessoas ${ }^{2}$. Esse conjunto foi usado para segmentar a vértebra da coluna no nosso experimento da COIFT sem largura ajustável (Subseção 4.4.4). Na Figura 4.12 podemos observar um exemplo de uma imagem pertencente a este conjunto.
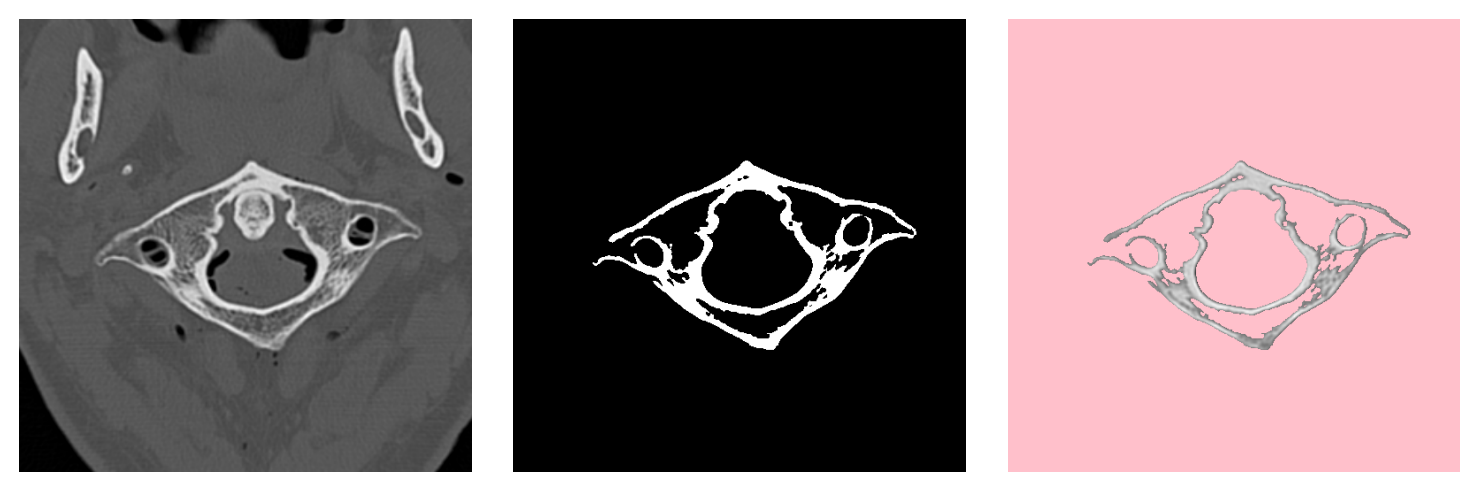

Figura 4.12: Exemplo de segmentação da vértebra da coluna.

\footnotetext{
${ }^{2}$ As imagens TC com os gabaritos correspondentes foram obtidas pelo Prof. Jayaram K. Udupa da Universidade da Pensilvânia (UPENN).
} 
- O segundo conjunto está composto por 44 imagens de Ressonância Magnética de 1 Tesla (RM-T1) de Phantoms, com gabarito conhecido, disponível no site BrainWeb ${ }^{3}$. Esse conjunto foi usado para segmentar a massa cinzenta cortical no nosso experimento da COIFT com largura ajustável (Subseção 4.4.5). Na Figura 4.13 podemos observar um exemplo de uma imagem pertencente a este conjunto.
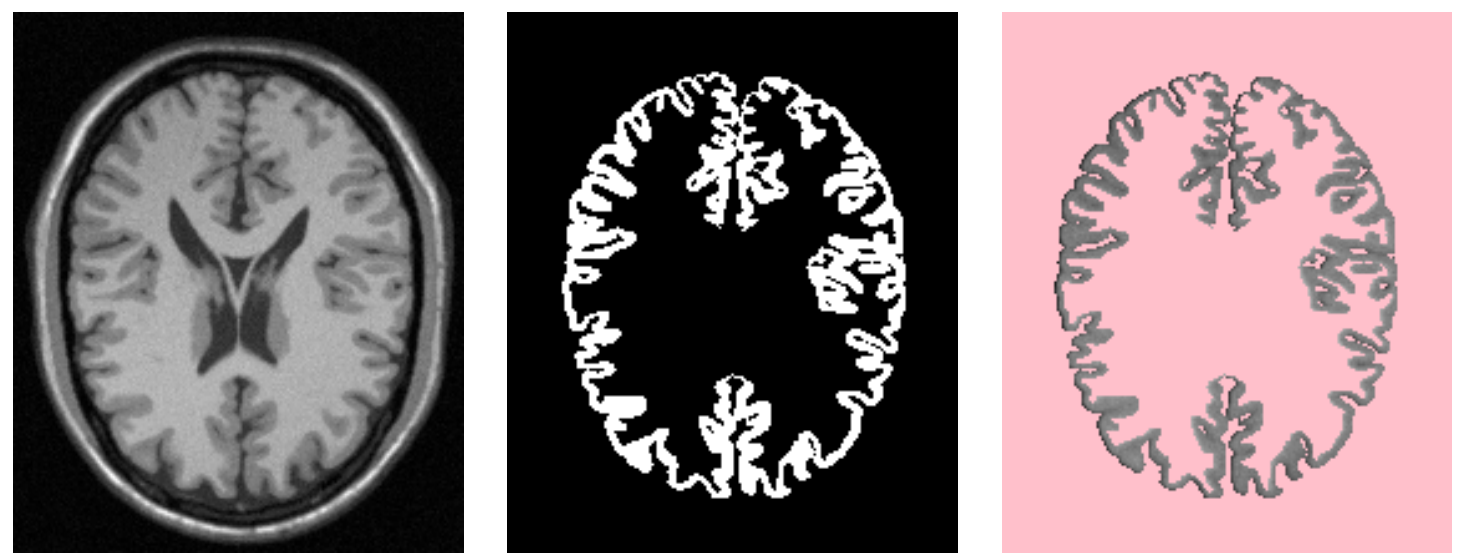

Figura 4.13: Exemplo de segmentação da massa cinzenta.

- O terceiro conjunto está composto por 50 imagens públicas de pássaros ${ }^{4}$, das quais desenhamos o gabarito por segmentação manual, disponíveis publicamente à comunidade ${ }^{5}$. Esse conjunto foi usado para segmentar um pássaro nas imagens no nosso experimento da COIFT com largura ajustável (Subseção 4.4.5). Na Figura 4.14 podemos observar um exemplo de uma imagem pertencente a este conjunto.
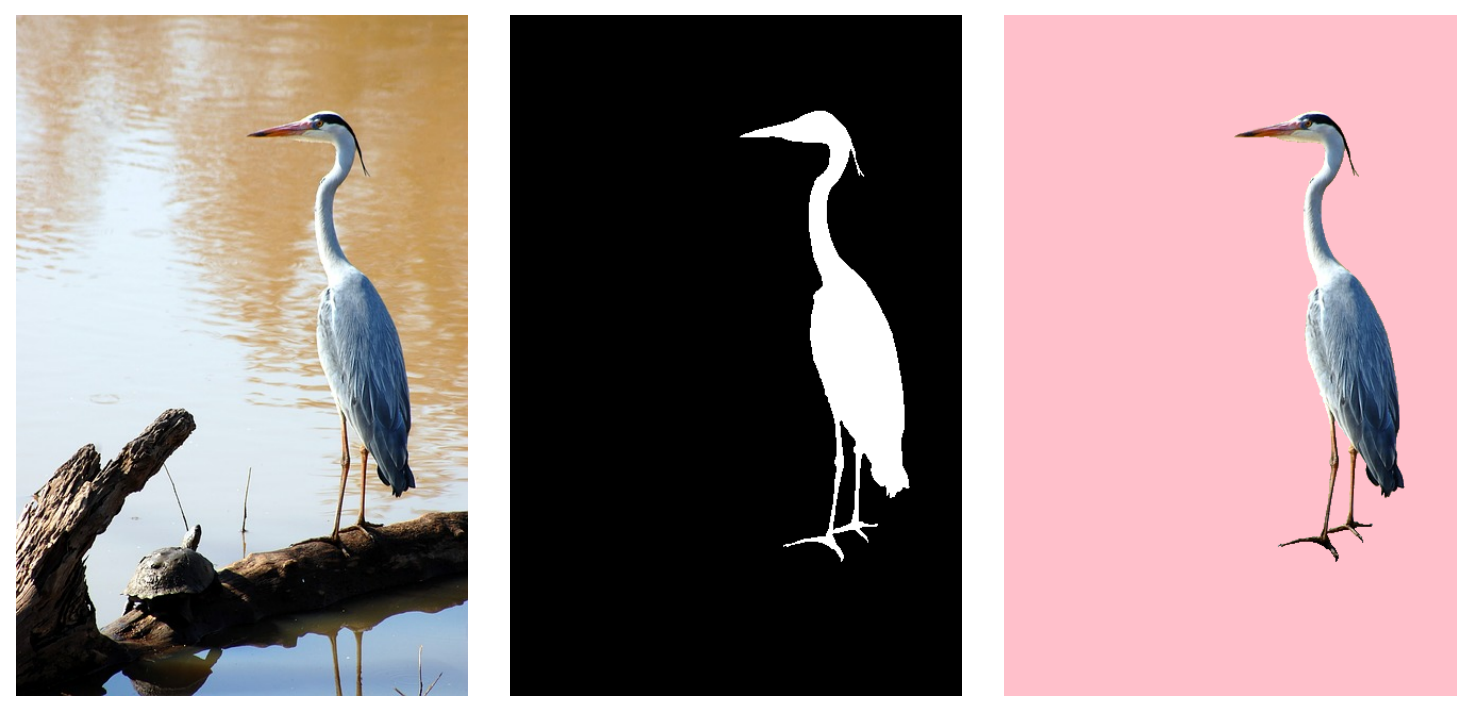

Figura 4.14: Exemplo de segmentação de um pássaro.

\footnotetext{
${ }^{3}$ URL: http://www.bic.mni.mcgill.ca/brainweb/

${ }^{4}$ URL: https://pixabay.com/

${ }^{5}$ URL: http://www.vision.ime.usp.br/ lucyacm/thesis/coift.html
} 
- O quarto conjunto está composto por 100 imagens públicas de pássaros ${ }^{4}$, das quais desenhamos o gabarito por segmentação manual, disponíveis publicamente à comunidade ${ }^{5}$. Esse conjunto foi usado para segmentar um pássaro nas imagens no nosso experimento da COIFT com largura ajustável considerando o tratamento de empates de energia (Subseção 4.4.5). Na Figura 4.15 podemos observar um exemplo de uma imagem pertencente a este conjunto.
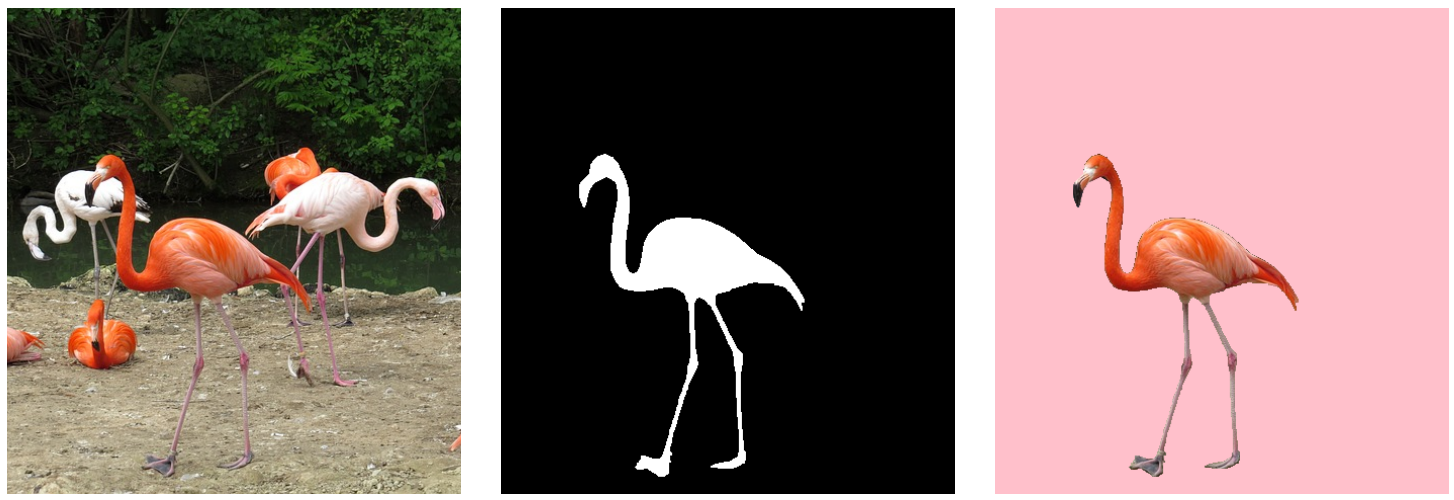

Figura 4.15: Exemplo de segmentação de um pássaro.

A diferença entre o terceiro e o quarto conjunto de imagens está no fato de que o quarto conjunto contém imagens cujo objeto alvo não possui buracos ou apresenta apenas buracos muito pequenos.

- O quinto conjunto está composto por 130 imagens públicas de aranhas, insetos e outros invertebrados ${ }^{4}$, das quais desenhamos o gabarito por segmentação manual, disponíveis publicamente à comunidade ${ }^{5}$. Esse conjunto foi usado para segmentar um inseto nas imagens no nosso experimento da COIFT com largura ajustável considerando o tratamento de empates de energia (Subseção 4.4.5). Na Figura 4.16 podemos observar um exemplo de uma imagem pertencente a este conjunto.
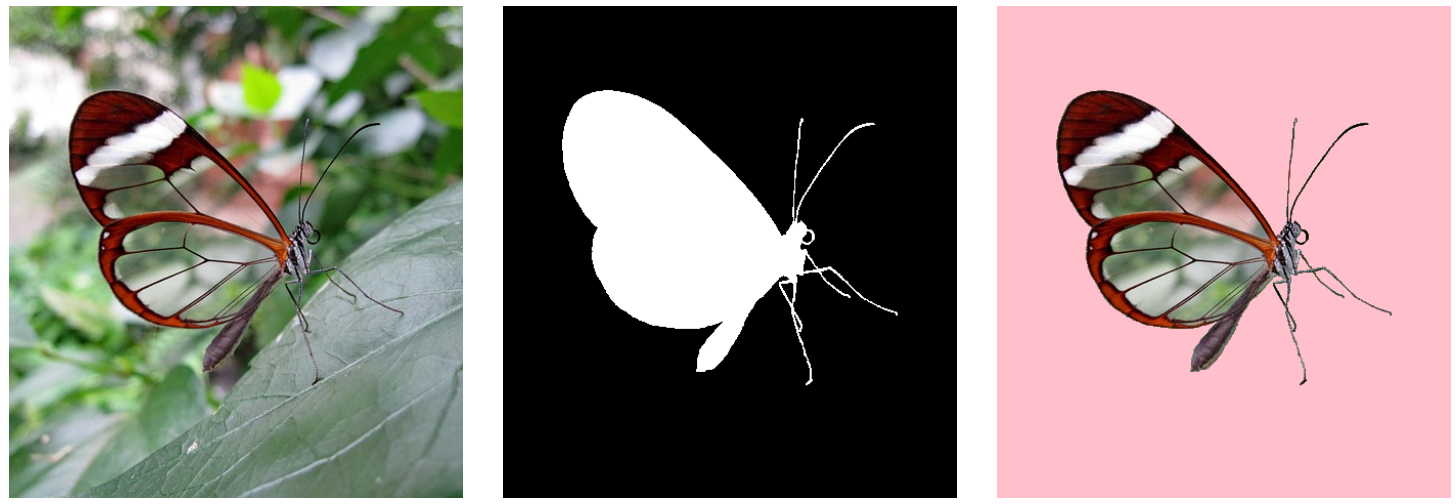

Figura 4.16: Exemplo de segmentação de um inseto.

\footnotetext{
${ }^{4}$ URL: https://pixabay.com/

${ }^{5}$ URL: http://www.vision.ime.usp.br/ lucyacm/thesis/coift.html
} 


\subsubsection{Seleção automática de marcadores}

Para gerar as sementes internas e externas de modo automático usamos duas abordagens, descritas a seguir:

- A primeira abordagem foi conduzida usando um usuário robô, como proposto por Gulshan et al. [Gulshan et al. (2010)], para simular a interação do usuário colocando pinceladas automaticamente para desempenhar a tarefa da segmentação de forma iterativa. Esta abordagem foi usada nos experimentos com os nossos três primeiros conjuntos de imagens (Subseção 4.4.1).

- A segunda abordagem foi conduzida usando os extremos do esqueleto e a erosão do objeto no gabarito da imagem para obter o conjunto de sementes internas $\mathcal{S}_{1}$, e no caso do conjunto de sementes externas $\mathcal{S}_{\boldsymbol{0}}$ foi realizada somente a erosão do fundo no gabarito das imagens para os mesmos raios de erosão considerados para obter $\mathcal{S}_{1}$. Esta abordagem foi usada nos nossos experimentos com os quarto e quinto conjuntos de imagens (Subseção 4.4.1). Na Figura 4.17 são mostrados dois exemplos de sementes calculadas automaticamente para uma imagem pertencente a nosso quarto conjunto de imagens.

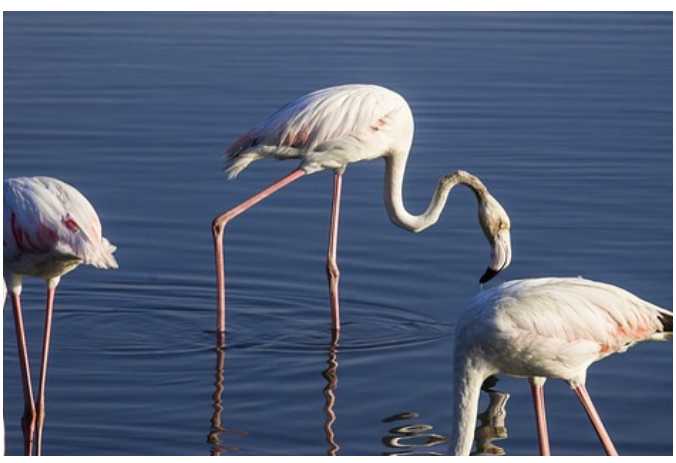

(a) $\hat{I}$

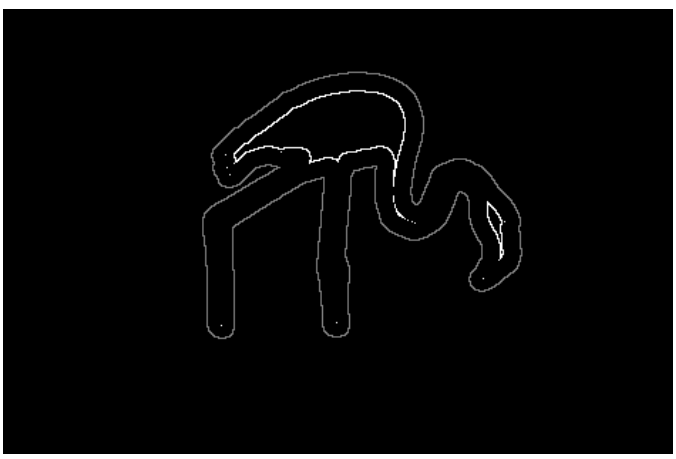

(c) raio $=6$

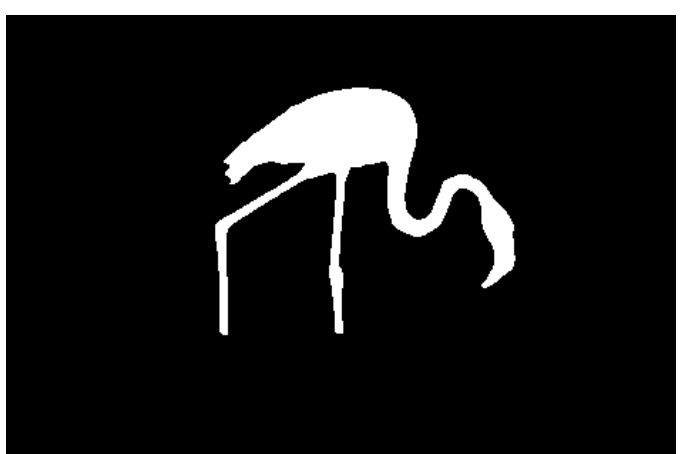

(b) Gabarito

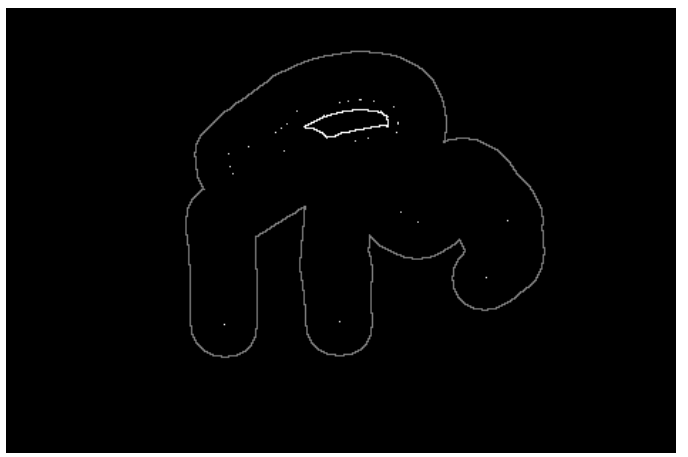

(d) raio $=20$

Figura 4.17: Exemplo dos conjuntos de sementes $\mathcal{S}_{1}$ e $\mathcal{S}_{\mathbf{0}}$ calculados automaticamente. (a) Imagem $\hat{I}$. (b) Gabarito da imagem $\hat{I}$. Em (b) e (c) são mostrados os conjuntos de sementes $\mathcal{S}_{1}$ (marcadores em branco) e $\mathcal{S}_{0}$ (marcadores em cinza), considerando um raio de erosão de 6 e 20, respectivamente. 
O resultado da segmentação pela OIFT e COIFT para a imagem da Figura 4.17a considerando um raio de erosão igual a 6 é mostrada na Figura 4.18.

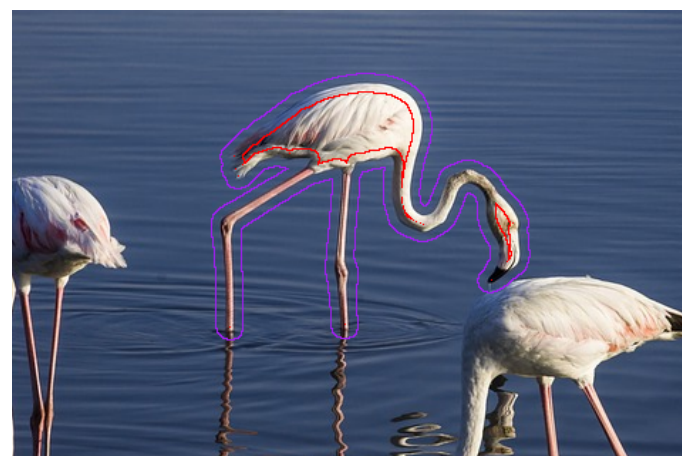

(a)

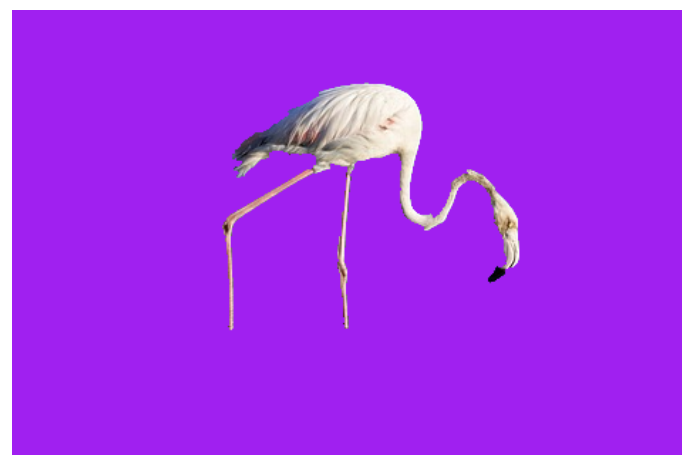

(c) $\operatorname{COIFT}(\gamma=1.0)$

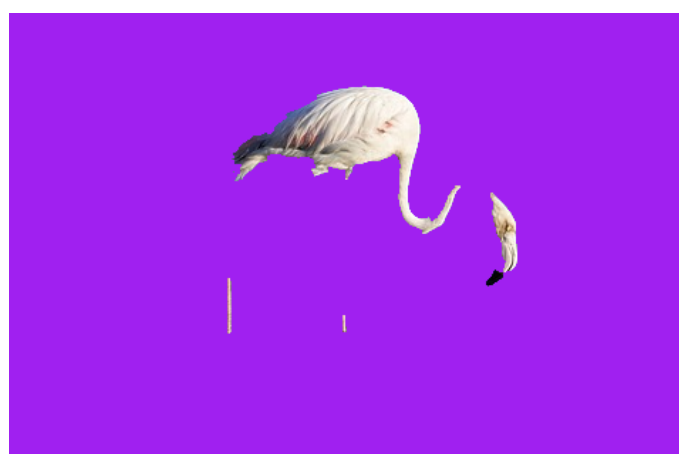

(b) OIFT

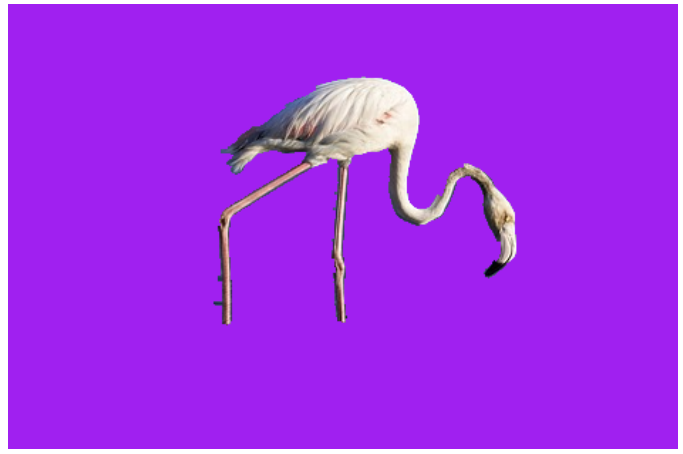

(d) $\operatorname{COIFT}(\gamma=3.0)$

Figura 4.18: Exemplo de segmentação pela (b) OIFT e (c,d) COIFT. Em (a) são mostrados as sementes internas (marcadores em vermelho) e as sementes externas (marcadores em roxo) calculadas automaticamente considerando os extremos do esqueleto e um raio de erosão de 6 (Figura 4.17b).

\subsubsection{Métodos comparados}

Nós comparamos COIFT contra outros métodos com tempos competitivos de execução (IRFC [Ciesielski et al. (2007)], OIFT [Miranda e Mansilla (2014)], ORFC+GC [Bejar e Miranda (2015); Ciesielski et al. (2013)]), a fim de mostrar que métodos baseados na minimização de $\varepsilon_{\infty}$ (ou equivalentemente na maximização de $E(\mathcal{O})$ ) podem se beneficiar do uso das restrições de conexidade.

A fim de salientar melhor as diferenças entre os métodos, não consideramos nenhum modelo prévio de distribuição de intensidades do objeto e fundo na atribuição dos pesos $\delta_{a}(\langle s, t\rangle)$, com base no método descrito em [Miranda et al. (2010)], visando um maior desafio.

\subsubsection{Experimentos usando COIFT sem largura ajustável}

No nosso primeiro experimento usamos as 40 imagens TC correspondentes a estudos da coluna cervical de 10 pessoas para segmentar a vértebra da coluna (Figuras 4.19), com $\alpha=0.5$ para todos os métodos com restrições de polaridade de borda. 
As Figuras 4.1 e 4.19 mostram o resultado da segmentação pela OIFT [Miranda e Mansilla (2014)] e COIFT para os mesmos marcadores selecionados pelo usuário, deixando claras as vantagens da COIFT.

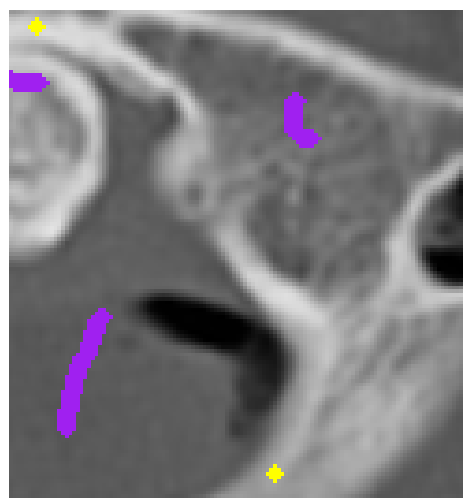

(a)

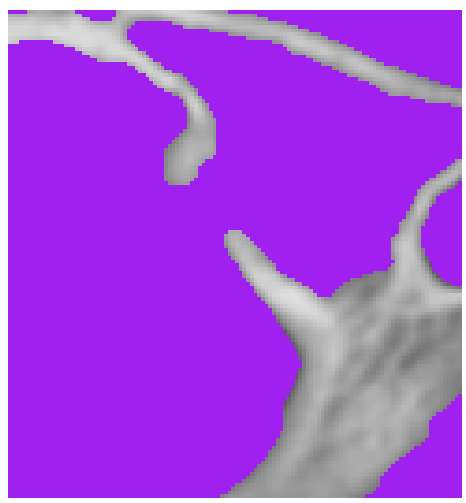

(b)

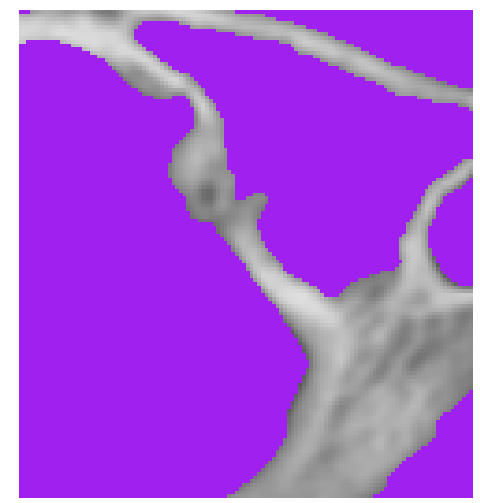

(c)

Figura 4.19: Exemplo de segmentação da vértebra da coluna via COIFT, mostrando a importância do uso da restrição de conexidade. (a) Imagem de entrada com marcadores selecionados pelo usuário. (b) Resultado da segmentação sem restrições de conexidade. (c) O resultado proposto pela COIFT garante um objeto conexo.

A Figura 4.20 mostra a curva experimental usando um usuário robô, o qual confirma os mesmos resultados para imagens da vértebra (Figura 4.19).
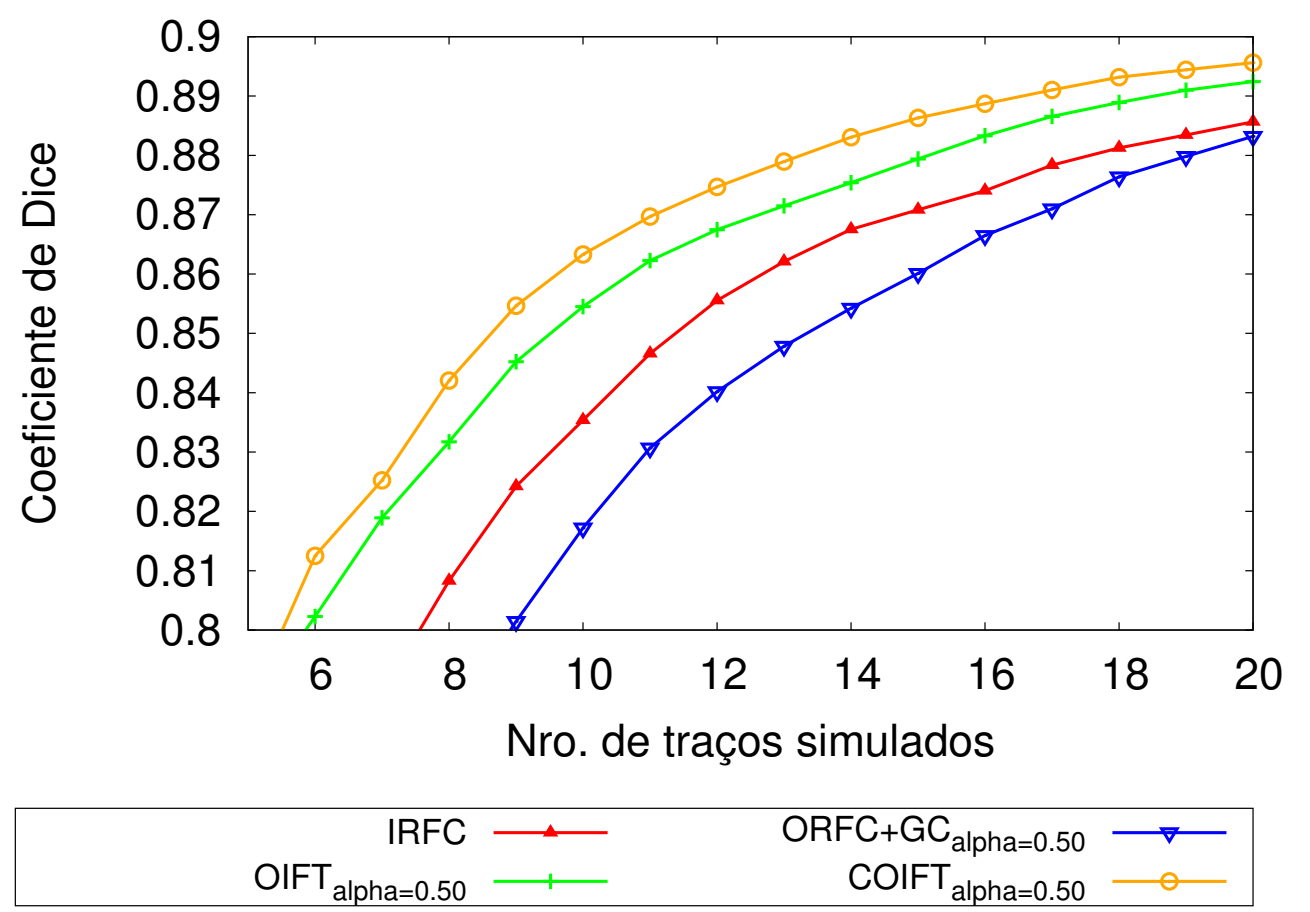

Figura 4.20: Curva de acurácia média da segmentação da vértebra da coluna por um usuário robô. 


\subsubsection{Experimentos usando COIFT com largura ajustável}

Como mencionado na Subseção 2.5.2, erros de segmentação de partes finas e alongadas dos objetos, não resultam em diferenças significativas na medida de avaliação usando o Coeficiente Dice, devido à quantidade pequena de pixels contidos nessas regiões. Assim, nos nossos experimentos usando a COIFT com largura ajustável usamos o erro de borda (Subseção 2.5.2) como medida de comparação entre os métodos avaliados a fim de diferenciar melhor seus desempenhos.

A seguir apresentamos os nossos experimentos usando a COIFT sem e com tratamento de empates de energia.

\section{COIFT sem tratamento de empates de energia}

No segundo experimento, usamos as 44 imagens RM-T1 de phantoms para segmentar a massa cinzenta cortical (Figura 4.21). A Figura 4.22 mostra as curvas de erro médio para todas as imagens usando um usuário robô para a segmentação da massa cinzenta cortical. Note que a COIFT tem o mais baixo erro entre todas as abordagens para $\gamma=2.0$.

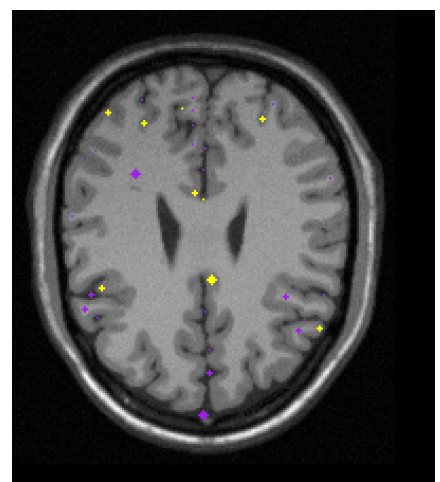

(a)

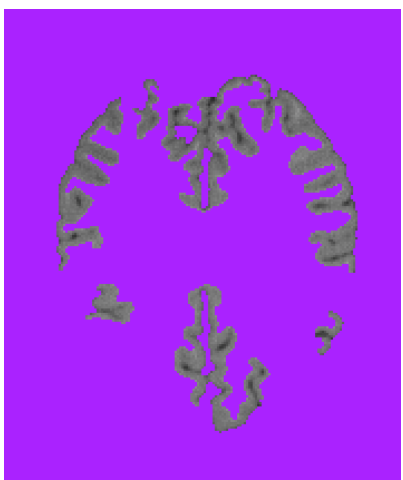

(b)

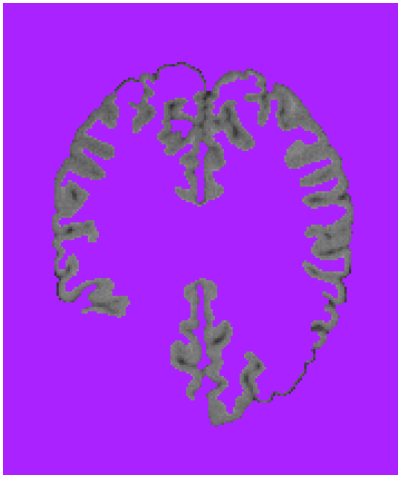

(c)

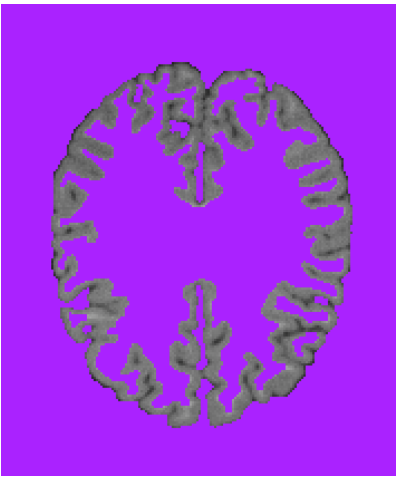

(d)

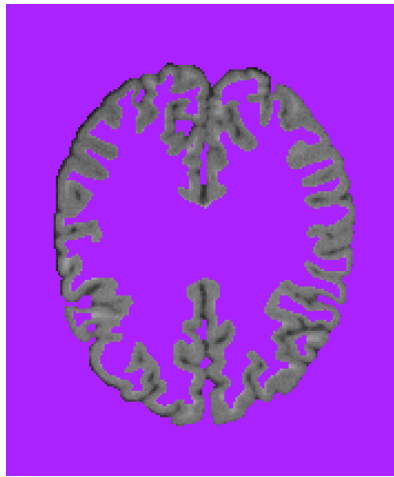

(e)

Figura 4.21: Segmentação da massa cinzenta cortical: (a) A imagem de entrada com marcadores selecionados pelo usuário. (b) Segmentação sem restrições de conexidade (OIFT com $\alpha=0.0$ ). (c) Segmentação pela COIFT com $\alpha=0.0$ e $\gamma=0.0$. (d) Segmentação pela COIFT com $\alpha=0.0$ e $\gamma=1.0$. (e) Segmentação pela COIFT com $\alpha=0.0$ e $\gamma=2.0$. Os resultados propostos pela COIFT garantem objetos conexos. 

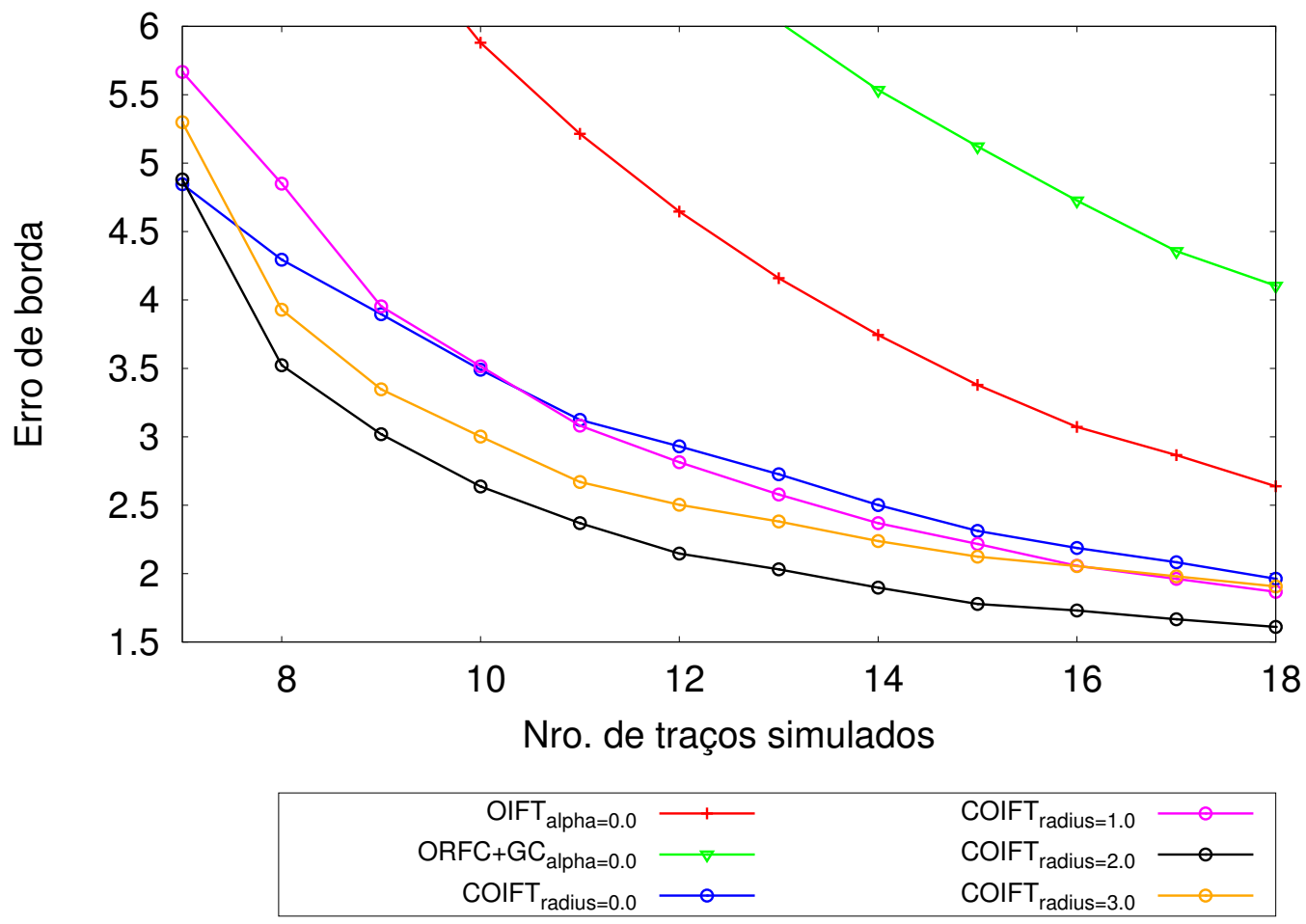

Figura 4.22: Curva de erro médio (erro de borda) usando um usuário robô para a segmentação da massa cinzenta cortical.

No terceiro experimento, usamos as 50 imagens públicas de pássaros (terceiro conjunto de imagens). A Figura 4.23 mostra os resultados de segmentação pela OIFT [Miranda e Mansilla (2014)] e COIFT com diferentes valores de raio $\gamma$ para os mesmos marcadores selecionados pelo usuário, deixando claras as vantagens da COIFT. Note que esse conjunto de imagens contém objetos com partes finas e alongadas. 


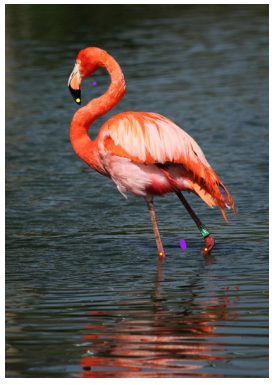

(a1)

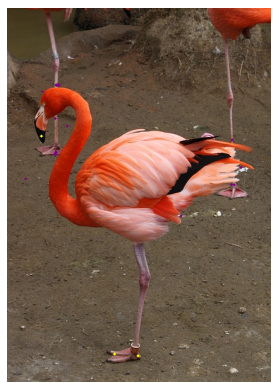

(b1)

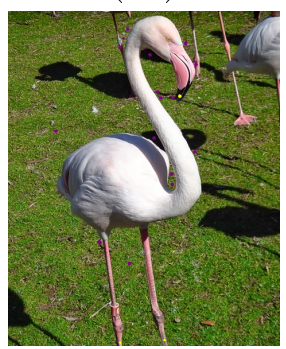

(c1)

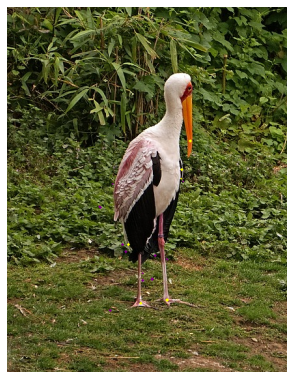

(d1)

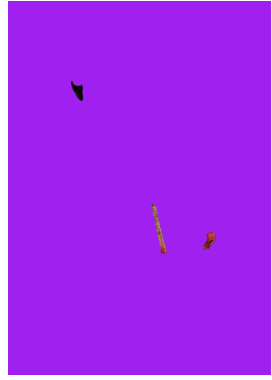

(a2)

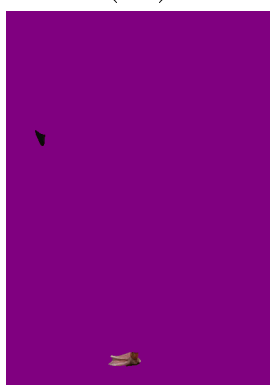

(b2)

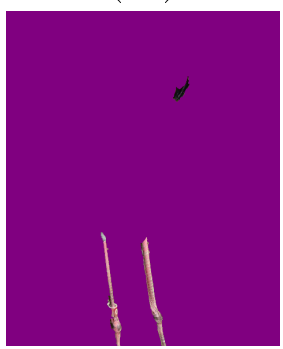

(c2)

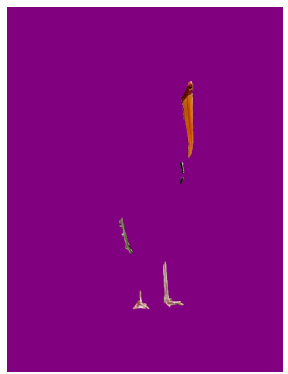

(d2)

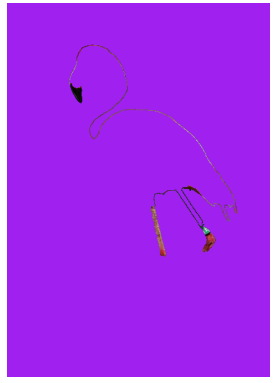

(a3)

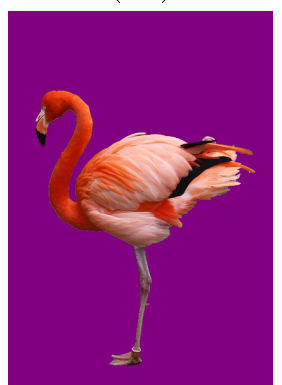

(b3)

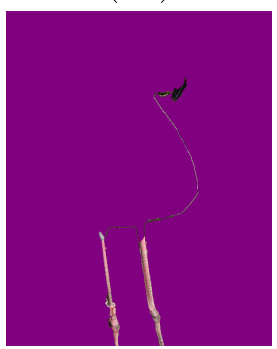

(c3)

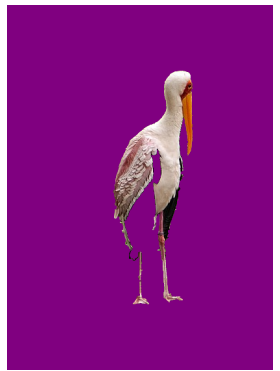

(d3)

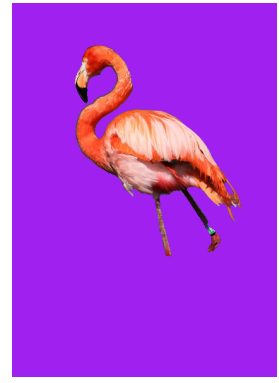

(a4)

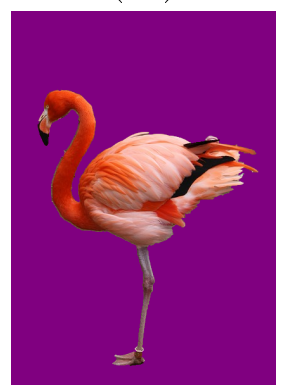

(b4)

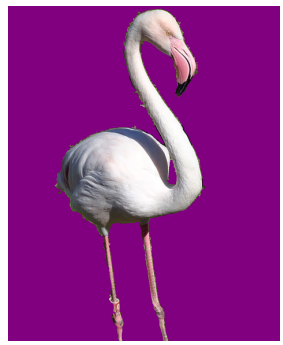

(c4)

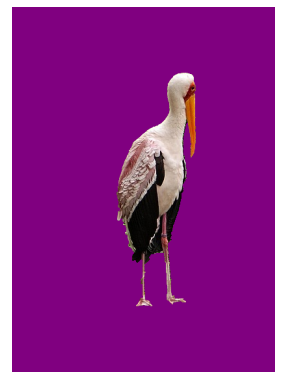

(d4)

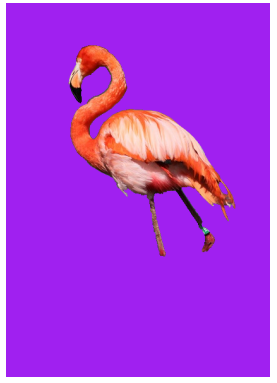

(a5)

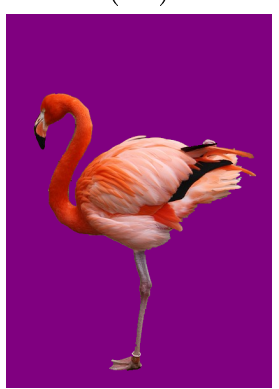

(b5)

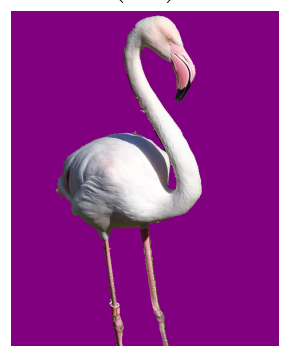

(c5)

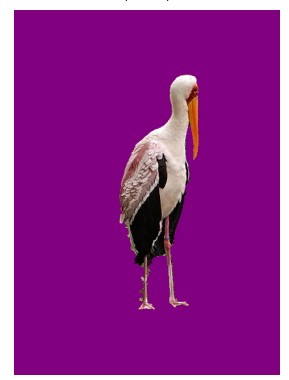

(d5)

Figura 4.23: Segmentação de diferentes pássaros : A primeira coluna representa as imagens de entrada com marcadores selecionados pelo usuário. A segunda coluna, segmentações sem restrições de conexidade (OIFT com $\alpha=0.0)$. A terceira coluna, segmentações com a COIFT $(\alpha=0.0$ e $\gamma=0.0)$. A quarta coluna, segmentações com a COIFT $(\alpha=0.0$ e $\gamma=1.0)$. A quinta coluna, segmentações com COIFT $(\alpha=0.0$ e $\gamma=2.0)$. Resultados propostos pela COIFT garantem objetos conexos.

Na Figura 4.24 mostramos as curvas de erro médio usando um usuário robô para segmentar o conjunto de imagens dos pássaros (terceiro conjunto de imagens). Para cada segmentação, o erro foi calculado como a distância média da borda do gabarito a partir da borda delineada. O método ORFC + GC [Bejar e Miranda (2015); Ciesielski et al. (2013)] não teve um bom desempenho neste conjunto de imagens porque não foi capaz de segmentar algumas partes finas e alongadas dos objetos. 


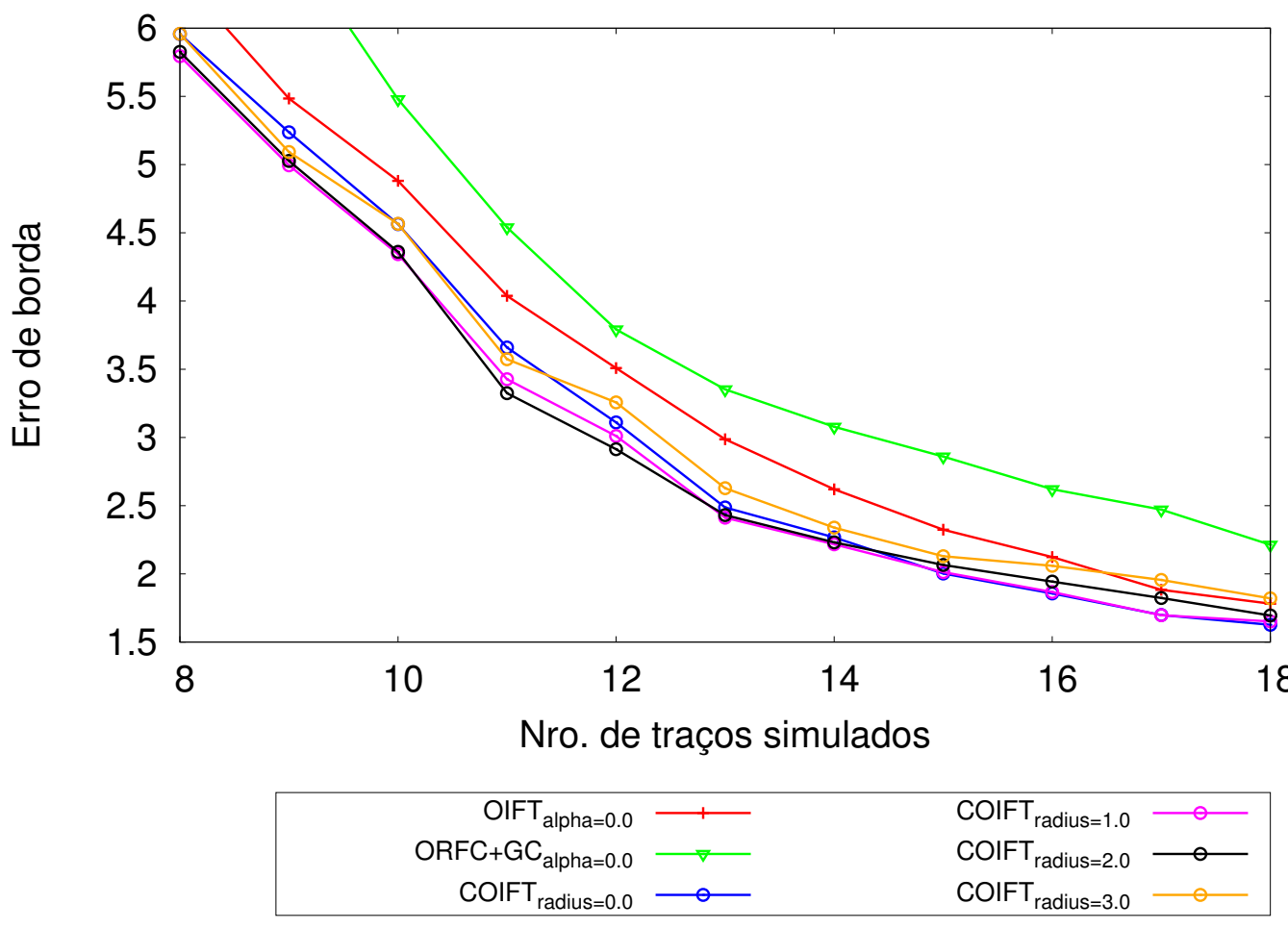

Figura 4.24: Curva de erro médio (erro de borda) usando um usuário robô para segmentar o conjunto de imagens dos pássaros (terceiro conjunto de imagens).

\section{COIFT com tratamento de empates de energia}

Nos dois últimos experimentos, detalhados a seguir, se fez uso da COIFT com a função $f_{\text {river }}^{\prime \prime}$ considerando os quatro componentes lexicográficos detalhados no tratamento de empates de energia (Subseção 4.3.2). Também, para os mesmos experimentos se fez uso dos extremos do esqueleto e erosão do objeto no gabarito da imagem para o cálculo automático das sementes internas (segunda abordagem na Subseção 4.4.2), sendo essa abordagem adequada para mostrar uma maior diferença entre os métodos de segmentação com e sem restrição de conexidade.

No quarto experimento, foram usadas as 100 imagens públicas de pássaros, sem sobreposição com o conjunto de imagens anterior usado no terceiro experimento. Na Figura 4.25 mostramos as curvas de erro médio usando os extremos do esqueleto conjuntamente com diferentes raios de erosão do objeto no gabarito das imagens para obter as sementes internas.

Finalmente, no quinto experimento usamos as 130 imagens públicas de insetos. Na Figura 4.26 mostramos as curvas de erro médio usando os extremos do esqueleto junto com diferentes raios de erosão do objeto no gabarito das imagens.

Note que, para valores elevados de $\gamma$, a restrição de $\gamma$-conexidade da COIFT gera resultados piores quando comparada com valores menores de $\gamma$ por conta de problemas nas partes finas do objeto. 


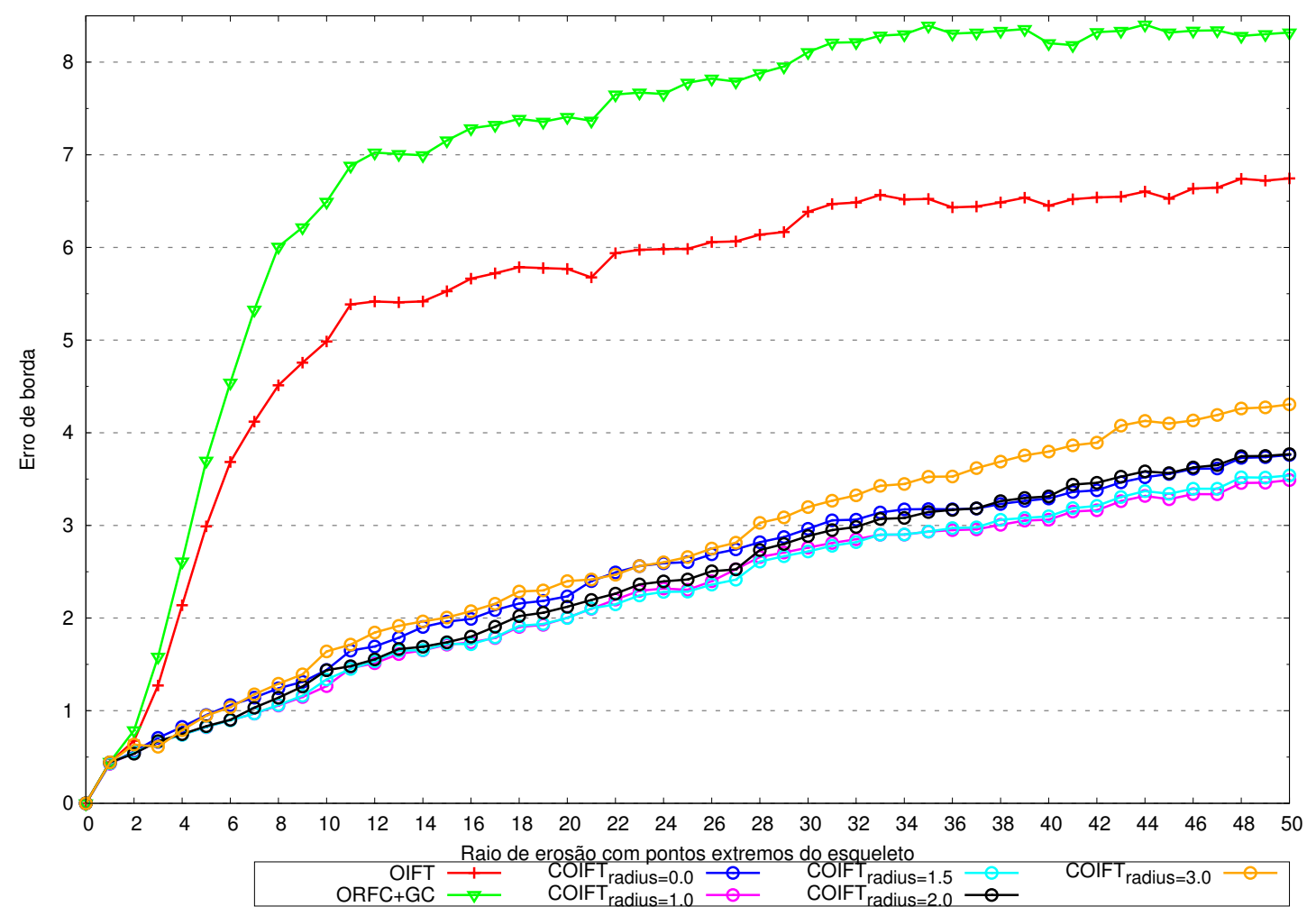

(a)

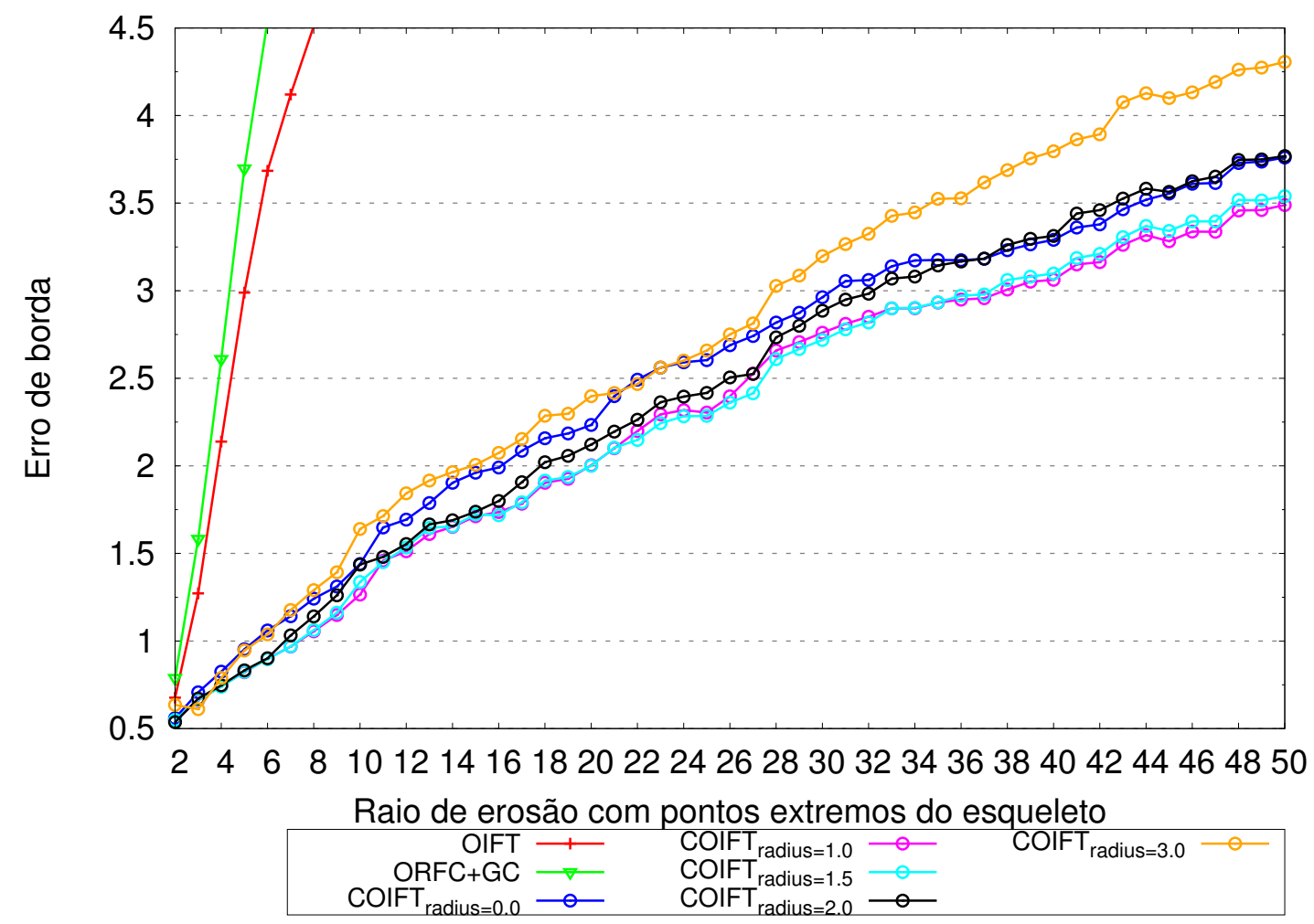

(b)

Figura 4.25: Curva de erro médio (erro de borda) usando os extremos do esqueleto e erosão do objeto no gabarito para segmentar o conjunto de imagens dos pássaros (quarto conjunto de imagens). Em (b) é mostrada uma versão expandida das curvas mostradas em (a). 


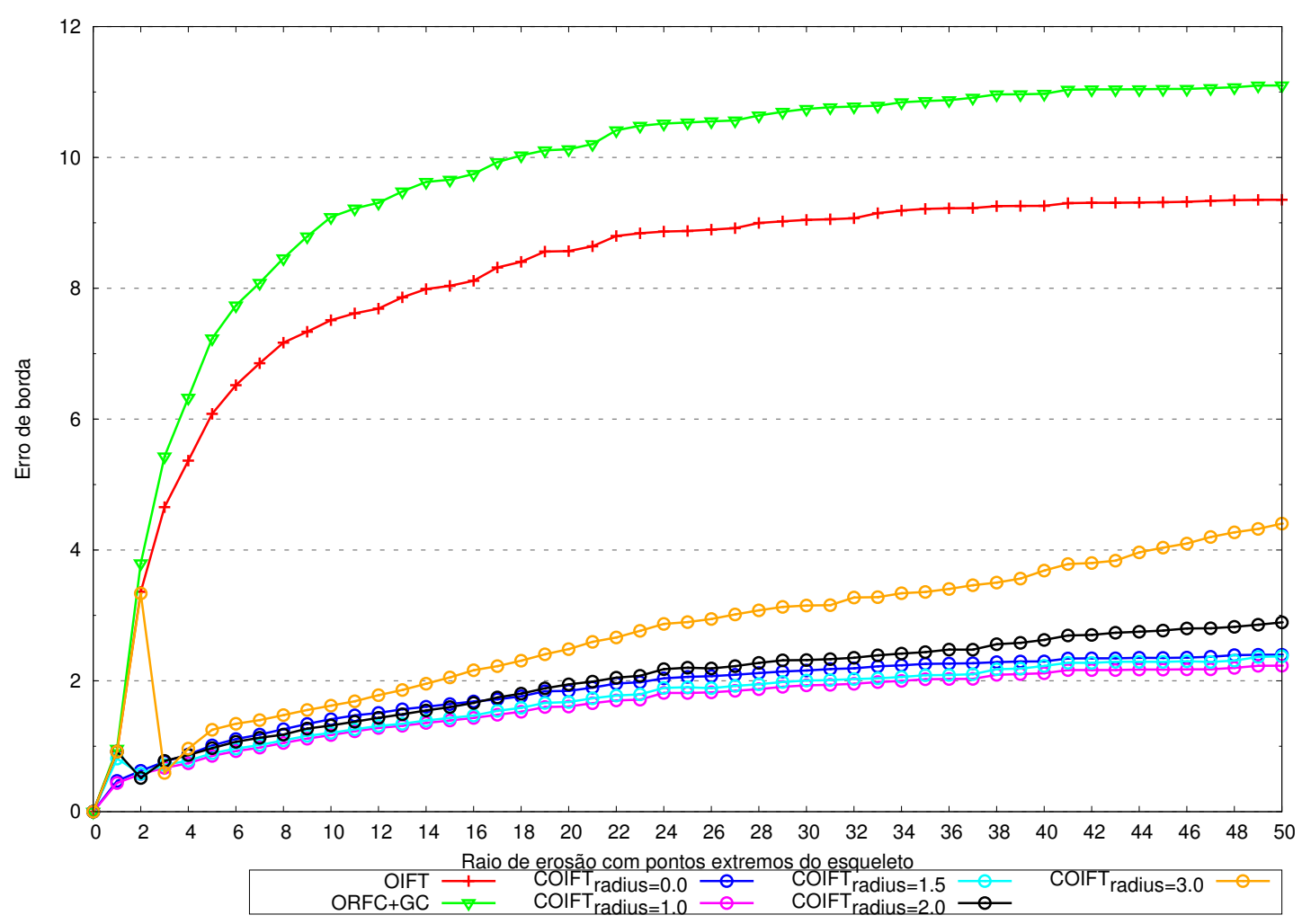

(a)

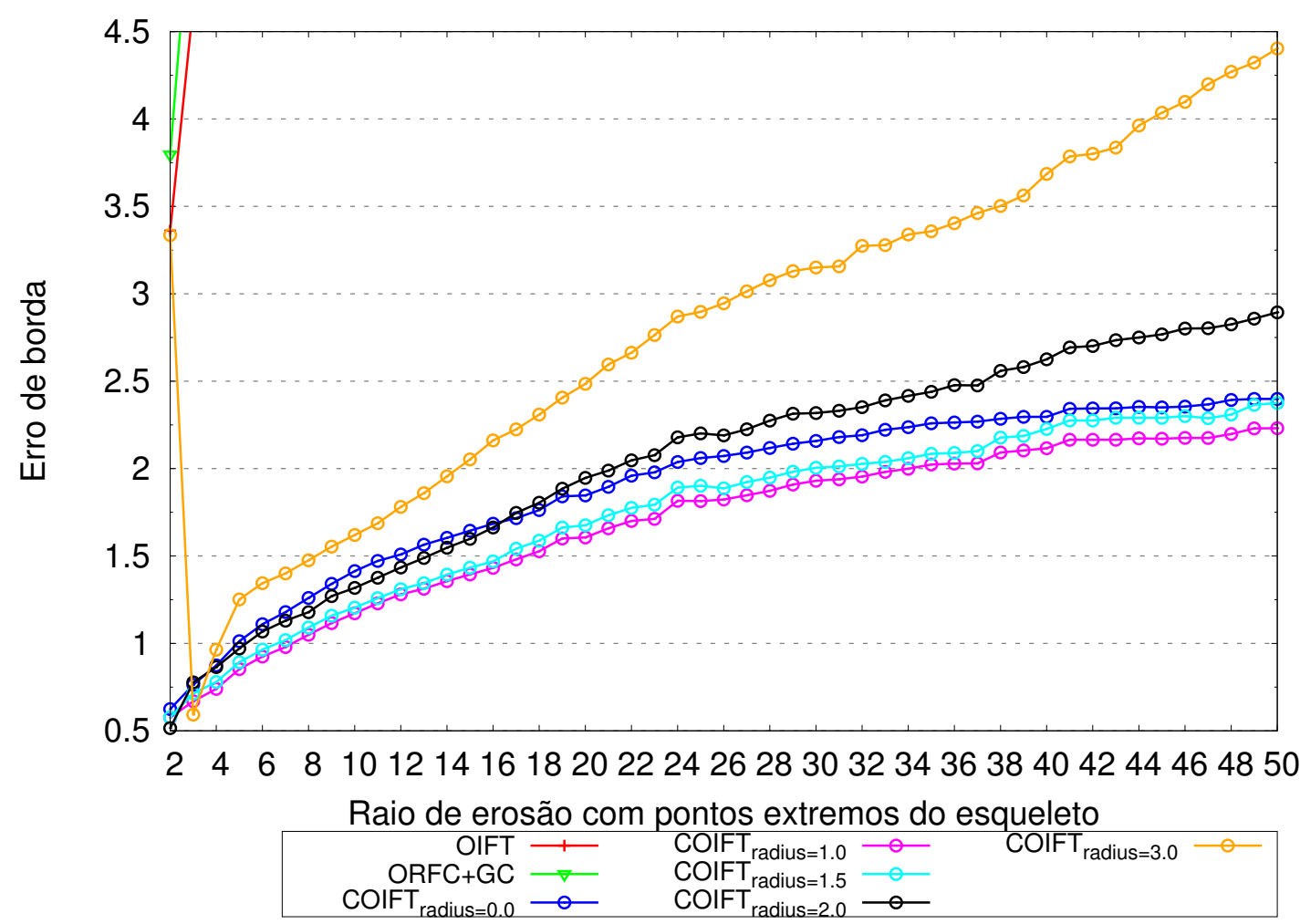

(b)

Figura 4.26: Curva de erro médio (erro de borda) usando os extremos do esqueleto e erosão do objeto no gabarito para segmentar o conjunto de imagens dos insetos. Em (b) é mostrada uma versão expandida das curvas mostradas em (a). 


\subsection{Observações}

\subsubsection{Restrições de conexidade para sementes do fundo}

O método da COIFT também pode ser usado para garantir fundos conexos, onde as restrições de conexidade devem ser aplicadas para sementes do fundo. Para isso, precisa-se inverter os papéis desempenhados pelas sementes internas e externas e aplicar a COIFT no grafo transposto $G^{T}$, como ilustrado na Figura 4.27. Note que nesse caso a COIFT garante objetos segmentados sem buracos.

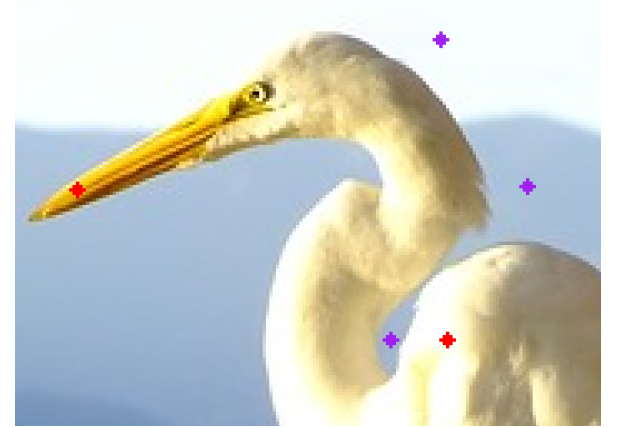

(a)

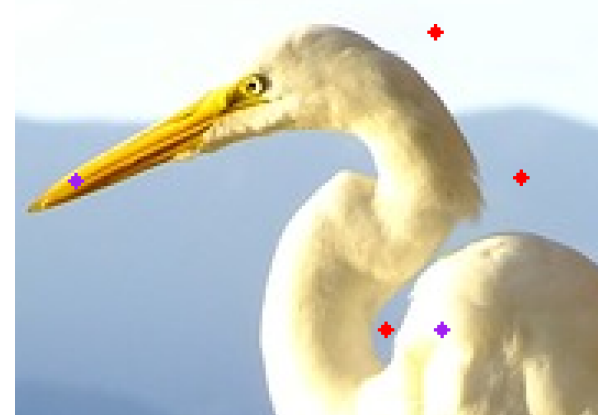

(c)

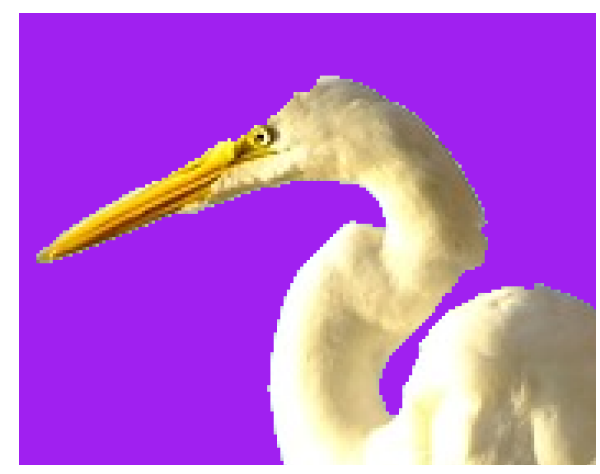

(e)

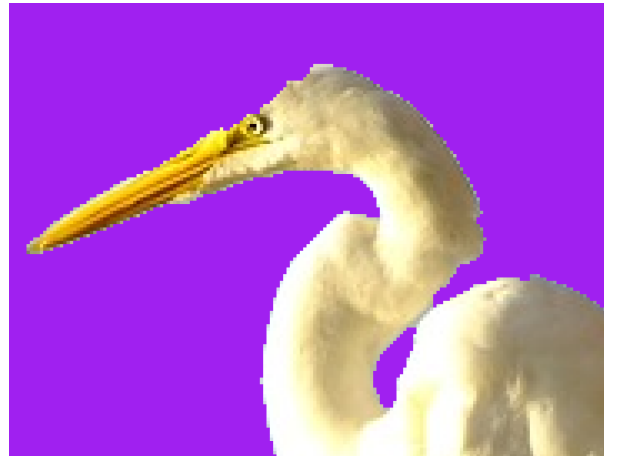

(b)

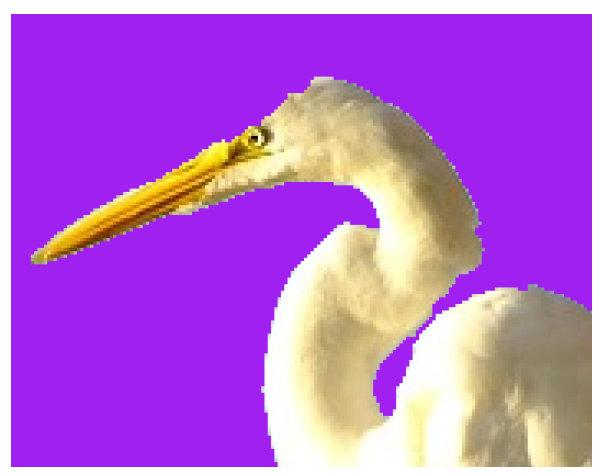

(d)

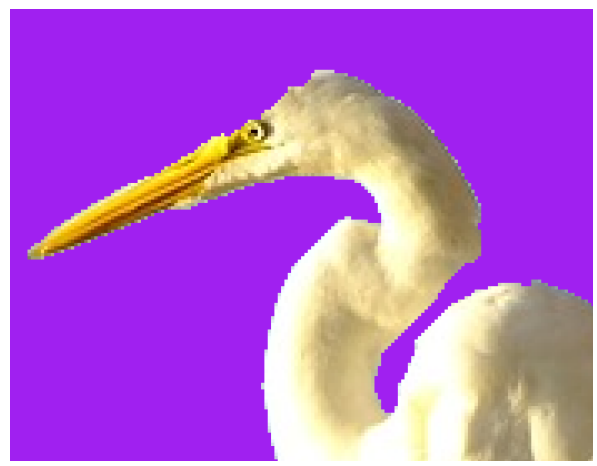

(f)

Figura 4.27: Exemplo de restrição de conexidade para sementes do fundo. (a) Imagem de entrada com marcadores selecionados pelo usuário. (b) Segmentação sem restrições de conexidade (OIFT com $\alpha=0.0)$. (c) Marcadores invertidos da imagem (a). (d) Segmentação com a COIFT ( $\alpha=0.0$ e $\gamma=0.0)$. (e) Segmentação com a COIFT $(\alpha=0.0$ e $\gamma=1.0)$. ( $f)$ Segmentação com a COIFT $(\alpha=0.0$ e $\gamma=2.0)$. Os resultados propostos pela COIFT garantem fundos conexos. 


\subsubsection{Casos intratáveis na COIFT}

Um caso em que a COIFT não garante um objeto conexo é dado quando as sementes do fundo dividem completamente as sementes do objeto como observado na Figura 4.28.

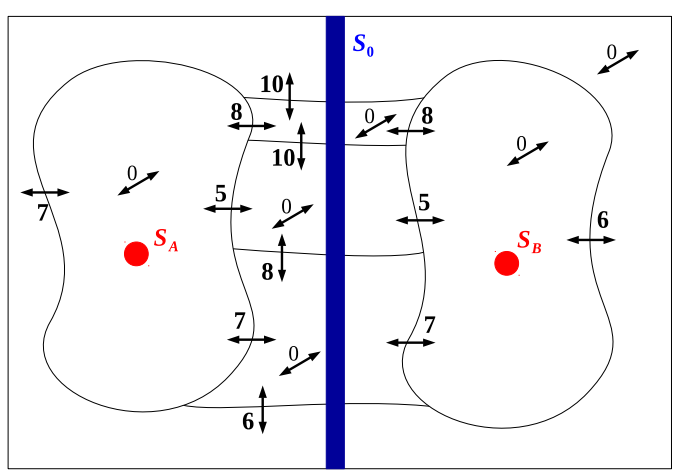

(a)

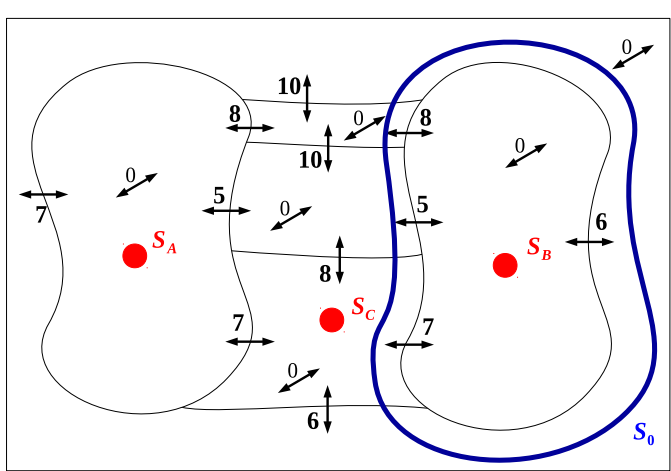

(b)

Figura 4.28: Exemplos de casos intratáveis via COIFT. (a) Imagem de entrada com marcadores selecionados pelo usuário, em que o conjunto de sementes internas é dado por $\mathcal{S}_{1}=\boldsymbol{S}_{\boldsymbol{A}} \cup \boldsymbol{S}_{\boldsymbol{B}}$. (b) Imagem de entrada com marcadores selecionados pelo usuário, em que o conjunto de sementes internas é $\mathcal{S}_{1}=\boldsymbol{S}_{\boldsymbol{A}} \cup \boldsymbol{S}_{\boldsymbol{B}} \cup \boldsymbol{S}_{\boldsymbol{C}}$. Note que o conjunto de sementes externas $\mathcal{S}_{0}$ divide a imagem, evitando qualquer conexão entre os conjuntos de sementes internas.

Note que nesse caso, a COIFT não fornece um objeto conexo devido ao fato de que não existe uma solução para esse caso em si, e não por um problema no algoritmo proposto. 


\section{Capítulo 5}

\section{Corte em grafo com funções não MI}

Existem casos nos quais o algoritmo da IFT sempre gera corretamente uma floresta de caminhos ótimos, os quais não são capturados pelas condições de uma função MI (Definição 5). Por exemplo, a IFT com a função de distância Euclidiana $f_{\text {euc }}$ (Equação 3.7) garante uma solução ótima se o número de sementes é menor que três (isto é, $|\mathcal{S}|<3$ ) ou se o raio $\rho$ de adjacência Euclidiana usado na definição dos arcos for suficientemente elevado [Falcão et al. (2004)]. Os caminhos gerados pela IFT com funções não MI, podem ter também a sua otimalidade definida por outros critérios, como é o caso da função $f_{\text {river }}$ de custo do riverbed [Miranda et al. (2012)].

Neste capítulo mostramos como uma classe particular da função de custo do riverbed, tal como usado pelo método da COIFT, garante um resultado ótimo de acordo com uma medida de corte em grafo, no contexto de competição de sementes.

\subsection{Corte ótimo com a função não MI $f_{\omega_{v}}$}

Para digrafos simétricos fortemente conexos, qualquer floresta enraizada nos vértices sementes é uma floresta de caminhos ótimos para a função de custo $f_{\text {river }}^{\prime}$ (Equação 4.13). Assim, o algoritmo da IFT sempre gerará um resultado correto.

Nesta seção a função $f_{\text {river }}^{\prime}$ será apresentada sob a forma da função $f_{\omega_{v}}$ (Equação 5.1), a fim de generalizar os resultados para qualquer peso $\omega_{v}$ associado aos vértices do digrafo,

$$
\begin{aligned}
f_{\omega_{v}}(\langle t\rangle) & = \begin{cases}0, & \text { se } t \in \mathcal{S} \\
+\infty, & \text { caso contrário }\end{cases} \\
f_{\omega_{v}}\left(\pi_{s} \cdot\langle s, t\rangle\right) & =\omega_{v}(t)
\end{aligned}
$$

tal que $\omega_{v}(t)$ representa o peso associado ao vértice $t \in \mathcal{I}$. Para fins de simplicidade usaremos $\omega_{v}(t)=I(t)$, sendo $I(t)$ o nível de intensidade do pixel $t$.

As Figuras 5.1a-c mostram um exemplo do cálculo de uma floresta de caminhos ótimos pelo Algoritmo 1 usando $f_{\omega_{v}}$. Na Figura 5.1c, está claro que os custos não são necessariamente uma função não decrescente ao longo do caminho, como requerido por $M 1$. Portanto, os resultados ótimos pelo Algoritmo 1 não são restritos à Definição 5.

Uma vez que qualquer floresta enraizada nos vértices sementes em $\mathcal{S}$ é uma floresta de caminhos ótimos para $f_{\omega_{v}}$ (Equação 5.1), à primeira vista parece que $f_{\omega_{v}}$ é uma função sem utilidade. No entanto, no contexto da segmentação de imagem por competição de sementes, a IFT com $f_{\omega_{v}}$ pode 
ser usada para produzir resultados similares ao watershed por marcadores no nível do pixel usando a função $f_{\text {peak }}$ (Equação 3.4) [Lotufo e Falcão (2000)], porém gerando um corte ótimo no grafo, conforme explicado a seguir.

Considere dois conjuntos de sementes $\mathcal{S}_{1}$ e $\mathcal{S}_{0}$, os quais estão compostos por pixels selecionados no objeto e fundo, respectivamente, tal que $\mathcal{S}=\mathcal{S}_{0} \cup \mathcal{S}_{1}$ na Equação 5.1. O objeto segmentado é definido a partir da floresta $P$ calculada pelo Algoritmo 1 como $\mathcal{O}^{P}=\left\{t \in \mathcal{I} \mid R\left(\pi_{t}^{P}\right) \in \mathcal{S}_{1}\right\}$. A segmentação resultante produz um corte ótimo $\mathcal{C}\left(\mathcal{O}^{P}\right)$ (Equação 2.4) no digrafo de acordo com o Teorema 4 (Figura 5.1), cuja prova é dada no Apêndice E.

Teorema 4 (Otimalidade do Corte da IFT com $f_{\omega_{v}}$ ) Para um digrafo simétrico fortemente conexo $G$, com dois conjuntos de sementes $\mathcal{S}_{0}$ e $\mathcal{S}_{1}$ dados, denota-se $\mathcal{U}\left(\mathcal{S}_{1}, \mathcal{S}_{0}\right)=\left\{\mathcal{O} \subseteq \mathcal{I} \mid \mathcal{S}_{1} \subseteq\right.$ $\left.\mathcal{O} \subseteq \mathcal{I} \backslash \mathcal{S}_{0}\right\}$ como o universo de todos os objetos possiveis satisfazendo as restrições de semente. $O$ algoritmo da IFT (Algoritmo 1) em $G$ com função de custo de caminho $f_{\omega_{v}}$ e $\mathcal{S}=\mathcal{S}_{0} \cup \mathcal{S}_{1}$ produz um objeto segmentado $\mathcal{O}^{P}$ o qual maximiza a energia $E(\mathcal{O})$ (Equação 5.2) entre todos os objetos em $\mathcal{U}\left(\mathcal{S}_{1}, \mathcal{S}_{0}\right)$. Isso é, $E\left(\mathcal{O}^{P}\right)=\max _{\mathcal{O} \in \mathcal{U}\left(\mathcal{S}_{1}, \mathcal{S}_{0}\right)} E(\mathcal{O})$.

$$
E(\mathcal{O})=\min _{\langle s, t\rangle \in \mathcal{C}(\mathcal{O})}\left\{\max \left\{\omega_{v}(s), \omega_{v}(t)\right\}\right\}
$$

\section{\begin{tabular}{ll|l|l|l}
0 & 2 & 1 & 2
\end{tabular} \begin{tabular}{lll|l|}
1 & 1 & 6 & 7
\end{tabular} $2 \quad 4 \quad 330$ \begin{tabular}{ll|l|l}
0 & 5 & 2 & 1 \\
\hline
\end{tabular}}

(a)

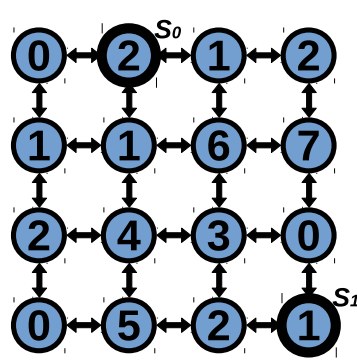

(b)

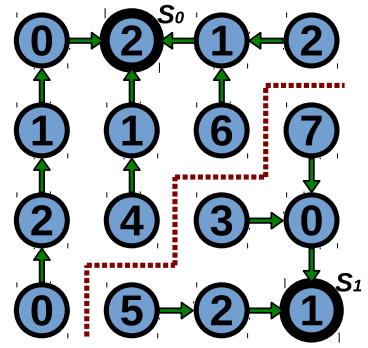

(c)

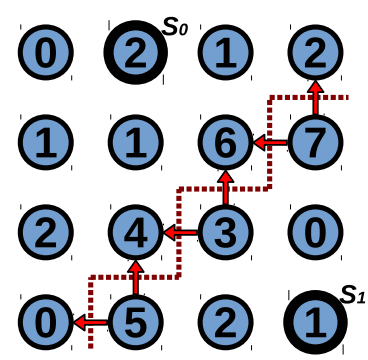

(d)

Figura 5.1: Exemplo da otimalidade do corte da IFT com a função $f_{\omega_{v}}$. (a) Uma imagem de entrada $\hat{I}$. (b) Um grafo com vizinhança-4 a partir de $\hat{I}$, em que os valores indicam $\omega_{v}(t)=I(t)$. (c) A floresta $P$ calculada pelo Algoritmo 1 com $f_{\omega_{v}}$, na qual os arcos indicam o predecessor $P(t)$ de cada vértice $t$. (d) $O$ corte resultante $\mathcal{C}(\mathcal{O})$ tem energia $E(\mathcal{O})=\min \{\max \{7,2\}, \max \{7,6\}, \max \{3,6\}, \max \{3,4\}, \max \{5,4\}, \max \{5,0\}\}=4$. 


\section{Capítulo 6}

\section{Conclusões}

Neste capítulo apresentamos algumas considerações finais do nosso trabalho, bem como algumas sugestões para trabalhos futuros.

\subsection{Considerações finais}

Neste trabalho, propomos um novo método chamado Transformada Imagem-Floresta Orientada Conexa (COIFT), que suporta uma largura mínima controlável pelo usuário da restrição de conexidade entre as sementes do objeto. Isso inclui o método da COIFT original [Mansilla et al. (2016)] como um caso particular quando $\gamma=0.0$.

Melhoramos o tratamento de empates na formulação da energia da COIFT e discutimos sua aplicação para evitar objetos com buracos, aplicando as restrições de conexidade para as sementes do fundo.

O nosso novo método incorpora satisfatoriamente restrições de conexidade na OIFT, preservando seu baixo custo de complexidade $O(N=|\mathcal{I}|)$ (quando $\mathcal{Q}$ é implementada usando bucket sorting [Falcão et al. (2004)]), uma vez que ele requer somente quatro execuções do algoritmo da IFT (Algoritmo 1, pág. 27).

Também, analisamos um caso particular da função de custo riverbed que garante um resultado ótimo de acordo com um critério de corte em grafo, bem como analisamos teoricamente a definição da IFT, apontando uma falha no seu artigo original e apresentando condições suficientes para sua corretude.

Além disso, durante o doutorado também conseguimos as seguintes contribuições:

- Foi disponibilizado um conjunto de 280 imagens públicas ${ }^{1}$ de pássaros, aranhas, insetos e outros invertebrados conjuntamente com seus gabaritos, obtidos por segmentação manual, na página web http://www.vision.ime.usp.br/ lucyacm/thesis/coift.html (Figura 6.1).

\footnotetext{
${ }^{1}$ Essas imagens estão liberadas em domínio público segundo a licença da Creative Commons CC0, disponíveis na página web https://pixabay.com/.
} 


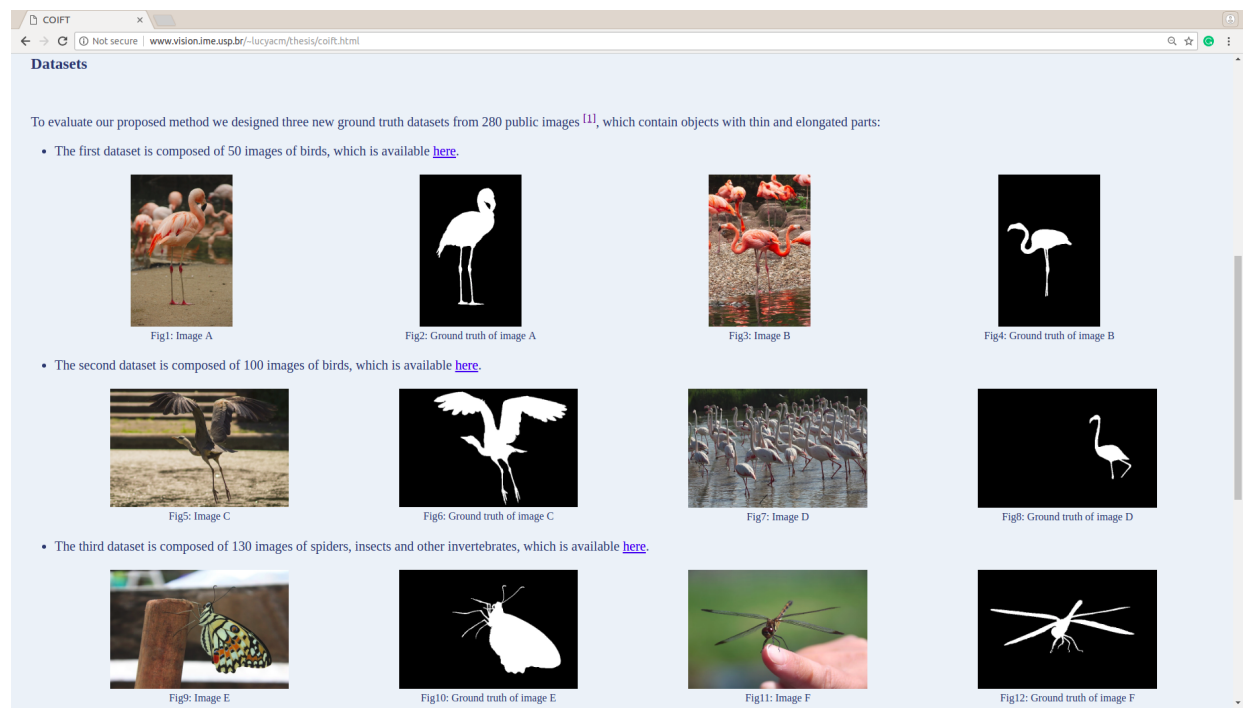

Figura 6.1: Página web com informação da COIFT

- A publicação dos artigos em conferências:

- Mansilla, L., Cappabianco, F. e Miranda, P. (ICIP-2016): "Oriented Image Foresting Transform Segmentation with Connectivity Constraints".

IEEE International Conference on Image Processing (ICIP).

Conceito A1.

- Mansilla, L. e Miranda, P. (SIBGRAPI-2016): "Oriented Image Foresting Transform Segmentation: Connectivity Constraints with Adjustable Width". XXVI Conference on Graphics, Patterns and Images (SIBGRAPI).

Conceito B1.

- Condori M., Mansilla L. e Miranda P. (ISMM-2017): "Bandeirantes: A Graphbased Approach for Curve Tracing and Boundary Tracking".

13th International Symposium on Mathematical Morphology (ISMM).

Conceito B1.

\subsection{Trabalhos futuros}

Observando as vantagens oferecidas pelo uso das restrições de conexidade, especialmente para segmentar objetos com partes finas e alongadas, como uma rede vascular, como trabalho futuro pretendemos:

- Estender a COIFT para aplicações em 3D a fim de auxiliar na segmentação de estruturas com anatomia complexa, tais como redes vasculares, para auxiliar o médico especialista na escolha da melhor opção de tratamento disponível no caso da presença de alguma patologia.

- Testar a COIFT em um arcabouço completo de segmentação automática.

- Estender a COIFT para múltiplos objetos a fim de melhorar os resultados da segmentação de objetos que representam estruturas finas. 


\section{Apêndice A}

\section{Prova de corretude da Proposição 1}

Proposição 1 Para um dado digrafo $G=\langle\mathcal{I}, \mathcal{A}, \omega\rangle$, considere uma função de custo de caminho monotônica $f$ com a propriedade de substituição. Seja $\mathcal{O}$ o conjunto de todos os pixels $t \in \mathcal{I}$, tal que existe um caminho hereditariamente ótimo $\pi_{t}$ para $f$. Em qualquer floresta de espalhamento $P$ calculada pelo algoritmo da IFT para $f$, todos os caminhos $\tau_{t}^{P} \operatorname{com} t \in \mathcal{O}$ são caminhos ótimos.

Prova: Seja $\pi_{t}=\left\langle t_{1}, t_{2}, \ldots, t_{i}, \ldots, t_{n}=t\right\rangle$ um caminho hereditariamente ótimo para $f$, e seja $\tau_{t}^{P}=\left\langle s_{1}, s_{2}, \ldots, s_{m}=t\right\rangle$ o caminho calculado na floresta de espalhamento $P$. Nós temos a seguinte prova por indução matemática:

- Base: Mostrar que a declaração é válida para $n=1$.

Nesse caso, temos $t=t_{1}$, e portanto $\pi_{t}=\left\langle t_{1}\right\rangle$. Como $f\left(\left\langle t_{1}\right\rangle\right)$ é um valor finito, $t_{1}$ é inserido na fila de prioridade $\mathcal{Q}$ (Linha 6 do Algoritmo 1, pág. 27). Também, como $f\left(\left\langle t_{1}\right\rangle\right)$ é ótimo , nós podemos garantir que ele não vai deixar $\mathcal{Q}$ (Linha 8) com um custo $V\left(t_{1}\right)$ pior do que $f\left(\left\langle t_{1}\right\rangle\right)$, assim, o caminho calculado $\tau_{t}^{P}$ só pode ser ótimo.

- Passo indutivo: Suponha a declaração é verdade para o caminho hereditariamente ótimo $\pi_{t_{i}}, i \geqslant 1$. Nós devemos provar que isso também é válido para o caminho hereditariamente ótimo $\pi_{t_{i+1}}=\pi_{t_{i}} \cdot\left\langle t_{i}, t_{i+1}\right\rangle$.

A partir da hipótese temos que o caminho $\tau_{t_{i}}^{P}$ em $P$ é ótimo. Dado que a propriedade de substituição é satisfeita para $f$, podemos concluir que:

$$
f\left(\tau_{t_{i}}^{P} \cdot\left\langle t_{i}, t_{i+1}\right\rangle\right)=f\left(\pi_{t_{i}} \cdot\left\langle t_{i}, t_{i+1}\right\rangle\right)=f\left(\pi_{t_{i+1}}\right)
$$

Assim, também podemos concluir que $\tau_{t_{i}}^{P} \cdot\left\langle t_{i}, t_{i+1}\right\rangle$ é ótimo.

Logo, por construção do algoritmo (Algoritmo 1, pág. 27), se $t_{i+1}$ ainda não foi conquistado, nós temos que esse caminho estendido $\tau_{t_{i}}^{P} \cdot\left\langle t_{i}, t_{i+1}\right\rangle$ será avaliado pelo Algoritmo 1 (Linhas 1014), e como este é ótimo, ele não será posteriormente substituído por qualquer outro caminho (como garantido pela desigualdade estrita na Linha 12). Se $t_{i+1}$ já foi conquistado seguindo um caminho diferente em $P$, seu custo não pode ser pior que $f\left(\pi_{t_{i+1}}\right)$, porque $f$ é uma função de custo de caminho monotônica. Assim, no final, $\tau_{t_{i+1}}^{P}$ será um caminho ótimo. 
APÊNDICE A 


\section{Apêndice B}

\section{Prova de corretude da Proposição 2 da COIFT}

Proposição 2 A energia ótima $E_{\boldsymbol{A} \cup \boldsymbol{B}}$ dentre todos os objetos em $\mathcal{U}\left(\boldsymbol{A} \cup \boldsymbol{B}, \mathcal{S}_{\mathbf{0}}\right)$, satisfaz $E_{\boldsymbol{A} \cup \boldsymbol{B}}=\min \left\{E_{\boldsymbol{A}}, E_{\boldsymbol{B}}\right\}$.

Prova: Vamos provar isso usando a prova por contradição e supor que:

$$
E_{\boldsymbol{A} \cup \boldsymbol{B}}<\min \left\{E_{\boldsymbol{A}}, E_{\boldsymbol{B}}\right\} .
$$

Logo, como a energia $E_{\boldsymbol{A} \cup \boldsymbol{B}}$ é ótima, temos que:

$$
E_{\boldsymbol{A} \cup \boldsymbol{B}}=\max _{\mathcal{O} \in \mathcal{U}\left(\boldsymbol{A} \cup B, \mathcal{S}_{0}\right)} E(\mathcal{O})
$$

Agora, considere dois objetos $\mathcal{O}_{\boldsymbol{A}} \in \mathcal{U}\left(\boldsymbol{A}, \mathcal{S}_{0}\right)$ e $\mathcal{O}_{\boldsymbol{B}} \in \mathcal{U}\left(\boldsymbol{B}, \mathcal{S}_{0}\right)$, tais que $E\left(\mathcal{O}_{A}\right)=E_{A}$ e $E\left(\mathcal{O}_{\boldsymbol{B}}\right)=E_{\boldsymbol{B}}$. Então, a união $\mathcal{D}=\mathcal{O}_{\boldsymbol{A}} \cup \mathcal{O}_{\boldsymbol{B}}$ é uma solução válida em $\mathcal{U}\left(\boldsymbol{A} \cup \boldsymbol{B}, \mathcal{S}_{0}\right)$, isto é, $\boldsymbol{A} \subset \mathcal{D}, \boldsymbol{B} \subset \mathcal{D}$ e $\mathcal{S}_{\mathbf{0}} \cap \mathcal{D}=\varnothing$.

Logo, como $\mathcal{D} \in \mathcal{U}\left(\boldsymbol{A} \cup \boldsymbol{B}, \mathcal{S}_{\mathbf{0}}\right)$, a partir da Equação B.2 podemos concluir que:

$$
E(\mathcal{D}) \leq E_{\boldsymbol{A} \cup \boldsymbol{B}}
$$

Por outro lado, da Equação 3.16 temos que:

$$
\begin{aligned}
& E(\mathcal{D})=\min _{\langle s, t\rangle \in \mathcal{C}(\mathcal{D})} \omega_{a}(\langle s, t\rangle) \\
& E(\mathcal{D})=\min _{\langle s, t\rangle \in \mathcal{C}\left(\mathcal{O}_{A} \cup \mathcal{O}_{B}\right)} \omega_{a}(\langle s, t\rangle) \\
& E(\mathcal{D})=\min \left\{\min _{\langle s, t\rangle \in \mathcal{C}\left(\mathcal{O}_{A}\right) \mid t \notin \mathcal{O}_{B}} \omega_{a}(\langle s, t\rangle), \min _{\langle s, t\rangle \in \mathcal{C}\left(\mathcal{O}_{B}\right) \mid t \notin \mathcal{O}_{A}} \omega_{a}(\langle s, t\rangle)\right\} \\
& E(\mathcal{D}) \geq \min \left\{\min _{\langle s, t\rangle \in \mathcal{C}\left(\mathcal{O}_{A}\right)} \omega_{a}(\langle s, t\rangle), \min _{\langle s, t\rangle \in \mathcal{C}\left(\mathcal{O}_{B}\right)} \omega_{a}(\langle s, t\rangle)\right\}=\min \left\{E_{\boldsymbol{A}}, E_{\boldsymbol{B}}\right\}
\end{aligned}
$$

Assim, da equação acima, temos que

$$
E(\mathcal{D}) \geq \min \left\{E_{\boldsymbol{A}}, E_{\boldsymbol{B}}\right\} .
$$


Mas como $E_{\boldsymbol{A} \cup \boldsymbol{B}}<\min \left\{E_{\boldsymbol{A}}, E_{\boldsymbol{B}}\right\}$ (Inequação B.1), temos que $E(\mathcal{D})>E_{\boldsymbol{A} \cup \boldsymbol{B}}$, o que leva a uma contradição de acordo com a Inequação B.3. 


\section{Apêndice C}

\section{Prova do teorema da otimalidade do corte pela COIFT}

Teorema 2 (Otimalidade do corte pela COIFT) Para dados dois conjuntos de sementes $\mathcal{S}_{\mathbf{1}}$ e $\mathcal{S}_{\mathbf{0}}$, qualquer objeto segmentado $\mathcal{O}_{\boldsymbol{c}}$ calculado pelo algoritmo da COIFT (Algoritmo 2) maximiza $E(\mathcal{O})$ (Equação 3.16$)$ dentre todos os resultados possíveis de segmentação em $\mathcal{U}_{\boldsymbol{c}}\left(\mathcal{S}_{\mathbf{1}}, \mathcal{S}_{\mathbf{0}}\right)$. Isto é:

$$
E\left(\mathcal{O}_{c}\right)=\max _{\mathcal{O}_{\mathcal{U}} \mathcal{U}_{c}\left(\mathcal{S}_{1}, \mathcal{S}_{0}\right)} E(\mathcal{O})
$$

Prova: Seja $\mathcal{O}_{\text {opt }}$ um objeto ótimo com energia máxima em $\mathcal{U}\left(\mathcal{S}_{1}, \mathcal{S}_{0}\right)$. A partir da Proposição 2 (pág. 44), podemos concluir que

$$
E\left(\mathcal{O}_{\text {opt }}\right)=\min _{s \in \mathcal{S}_{1}} E_{\{s\}}
$$

De fato, também podemos deduzir que

$$
E\left(\mathcal{O}_{\text {opt }}\right)=\min _{t \in \mathcal{O}_{\text {opt }}} E_{\{t\}}
$$

uma vez que $\mathcal{O}_{\text {opt }} \in \mathcal{U}\left(\mathcal{O}_{\text {opt }}, \mathcal{S}_{0}\right) \subseteq \mathcal{U}\left(\mathcal{S}_{1}, \mathcal{S}_{0}\right)$ e $E\left(\mathcal{O}_{\text {opt }}\right)=\max _{\mathcal{O} \in \mathcal{U}\left(\mathcal{S}_{1}, \mathcal{S}_{0}\right)} E(\mathcal{O})$, o que implica que

$$
E\left(\mathcal{O}_{\text {opt }}\right)=\max _{\mathcal{O} \in \mathcal{U}\left(\mathcal{O}_{\text {opt }}, \mathcal{S}_{0}\right)} E(\mathcal{O})
$$

Seja $\mathcal{U}_{\boldsymbol{c}}\left(\boldsymbol{S}_{1}, \boldsymbol{S}_{\mathbf{0}}\right)$ o conjunto de todos os objetos conexos possíveis da segmentação a partir das sementes $\mathcal{S}_{1}$ e $\mathcal{S}_{\mathbf{0}}$ (Equação 4.11) e $\mathcal{O}_{\boldsymbol{o p t}}^{\boldsymbol{c}} \in \mathcal{U}_{\boldsymbol{c}}\left(\boldsymbol{S}_{\mathbf{1}}, \boldsymbol{S}_{\mathbf{0}}\right)$ uma solução ótima tal que

$$
E\left(\mathcal{O}_{\text {opt }}^{c}\right)=\max _{\mathcal{O} \in \mathcal{U}_{c}\left(\boldsymbol{S}_{1}, S_{0}\right)} E(\mathcal{O})
$$

Dado que um corte ótimo num espaço de busca menor com mais restrições não pode gerar 
valores de energia melhores que a solução ótima em um espaço com menos restrições, temos que:

$$
E\left(\mathcal{O}_{\text {opt }}^{c}\right) \leqslant E\left(\mathcal{O}_{\text {opt }}\right)
$$

Dado que $\mathcal{O}_{\text {opt }}^{\boldsymbol{c}}$ é conexo, então existe um conjunto conexo de pixels $\mathcal{S}_{1}^{c}$, composto pelos pixels em $\mathcal{S}_{1}$ estendido por caminhos que os interligam, tal que $\mathcal{S}_{1} \subseteq \mathcal{S}_{1}^{c} \subseteq \mathcal{O}_{\text {opt }}^{c}$. Assim, temos que:

$$
E\left(\mathcal{O}_{\text {opt }}^{c}\right)=\max _{\mathcal{O} \in \mathcal{U}_{c}\left(\mathcal{S}_{1}, \mathcal{S}_{0}\right)} E(\mathcal{O})=\max _{\mathcal{O} \in \mathcal{U}_{c}\left(\mathcal{S}_{1}^{c}, \mathcal{S}_{0}\right)} E(\mathcal{O})=\max _{\mathcal{O} \in \mathcal{U}\left(\mathcal{S}_{1}^{c}, \mathcal{S}_{0}\right)} E(\mathcal{O})
$$

e da Proposição 2 (pág. 44) podemos deduzir que

$$
E\left(\mathcal{O}_{o p t}^{c}\right)=\min _{t \in \mathcal{S}_{1}^{c}} E_{\{t\}}=E_{\mathcal{S}_{1}^{c}}
$$

Portanto, um conjunto de sementes interligadas $\mathcal{S}_{1}^{c}$ passando por dentro de $\mathcal{O}_{\text {opt }}^{c}$ é suficiente para garantir um resultado ótimo, porém como $\mathcal{O}_{\text {opt }}^{\boldsymbol{c}}$ é desconhecido, devemos selecionar as sementes de modo a maximizar $\min _{t \in \mathcal{S}_{1}^{c}} E_{\{t\}}$. Dado que $\mathcal{S}_{1} \subseteq \mathcal{S}_{1}^{c}$ é fixo, devemos mais precisamente selecionar $\mathcal{S}_{1}^{c} \backslash \mathcal{S}_{1}$ de modo a maximizar $\min _{t \in \mathcal{S}_{1}^{c} \backslash \mathcal{S}_{1}} E_{\{t\}}$. Essa seleção é garantida pelo cálculo das interconexões pela função do riverbed, conforme explicado abaixo.

Para isso, vamos verificar os passos do algoritmo da COIFT (Algoritmo 2, pág. 47).

Primeiro, nas Linhas 2-3, o algoritmo da COIFT (Algoritmo 2) calcula o mapa de energias $\mathcal{E}$, tal que

$$
\mathcal{E}(t)=E_{\{t\}}=V_{o p t}^{f_{\max }^{\# \mathcal{S}_{0}}}(t)
$$

para todo $t \in \mathcal{I}$, o que requer uma execução do algoritmo da IFT com $f_{\max }^{\# \mathcal{S}_{0}}$ usando só as sementes em $\mathcal{S}_{0}$ para inicializar os custos dos caminhos triviais (Linha 1 ).

Logo, na Linha 4, o algoritmo da COIFT com o algoritmo NewObjSeeds (Algoritmo 3, pág. 47) interconecta sementes não conexas em $\mathcal{S}_{1}$ através de pixels com valores altos de energia $E_{\{t\}}$, fazendo uso da função de custo $f_{\text {river }}^{\prime}$ (Equação 4.13), a qual é um caso particular da função $f_{\text {river }}$ (Equação 3.20), tal que $\omega_{v}(t)=K_{1}-E_{\{t\}}, K_{1}=\max _{t \in \mathcal{I}} E_{\{t\}}$ e $s^{*}$ é um vértice arbitrário em $\mathcal{S}_{\mathbf{1}}$.

Assim, um caminho riverbed $\tau_{r \sim t}$ (caminho calculado pelo Algoritmo 1 (pág. 27) usando $f_{\text {river }}^{\prime}$ ) a partir do vértice $r=s^{*}$ até $t$, sempre atravessa uma sequência de vértices intermediários $t_{i}$, para os quais o valor máximo de $\omega_{v}\left(t_{i}\right)=K_{1}-E_{\left\{t_{i}\right\}}$ é minimizado ao longo de qualquer parte dele entre outros caminhos alternativos possíveis [Miranda et al. (2012)]. Consequentemente, um caminho riverbed pela $f_{\text {river }}^{\prime}$ sempre busca níveis mais altos de energia $E_{\left\{t_{i}\right\}}$ que garante um corte ótimo como desejado.

Na Linha 1 do Algoritmo 3 (pág. 47), um vértice arbitrário em $\mathcal{S}_{1}$ é selecionado para ser usado como $s^{*}$ para o cálculo da IFT com função de custo $f_{\text {river }}^{\prime}$, gerando uma floresta de caminhos $P_{\text {river }}$, tal que para todo $t \in \mathcal{S}_{1} \backslash\left\{s^{*}\right\}$, são selecionados todos os pixels no caminho $\pi_{t}^{P_{\text {river }}}$ para compor um novo conjunto conexo de sementes internas $\mathcal{S}_{1}^{c}$ (Linha 4 do Algoritmo 2, pág. 47).

Finalmente, na Linha 5, a COIFT calcula o método da OIFT com $f^{0^{\top}}$ (Equação 3.19) a partir dos conjuntos de sementes $\mathcal{S}_{\mathbf{1}}^{\boldsymbol{c}}$ e $\mathcal{S}_{\mathbf{0}}$, gerando um resultado final, o qual é garantido ser um objeto 
conexo $\mathcal{O}_{c}$, tal que

$$
E\left(\mathcal{O}_{c}\right)=\max _{\mathcal{O} \in \mathcal{U}\left(\mathcal{S}_{1}^{\mathrm{c}}, \mathcal{S}_{0}\right)} E(\mathcal{O})=\max _{\mathcal{O} \in \mathcal{U}_{c}\left(\mathcal{S}_{1}^{\mathrm{c}}, \mathcal{S}_{0}\right)} E(\mathcal{O})=\max _{\mathcal{O} \in \mathcal{U}_{c}\left(\mathcal{S}_{1}, \mathcal{S}_{0}\right)} E(\mathcal{O})
$$




\section{Apêndice D}

\section{Prova do teorema da otimalidade do corte pela COIFT com largura ajustável}

Teorema 3 (Otimalidade do corte pela COIFT com largura ajustável) Para dados dois conjuntos de sementes $\mathcal{S}_{\mathbf{1}}$ e $\mathcal{S}_{\mathbf{0}}$, qualquer objeto segmentado $\mathcal{O}_{\boldsymbol{c}}^{\gamma}$ calculado pelo algoritmo da COIFT com largura ajustável (Algoritmo 4) maximiza $E(\mathcal{O})$ (Equação 3.16) dentre todos os resultados possíveis de segmentação em $\mathcal{U}_{c}^{\gamma}\left(\mathcal{S}_{1}, \mathcal{S}_{0}\right)$. Isto é:

$$
E\left(\mathcal{O}_{c}^{\gamma}\right)=\max _{\mathcal{O} \in \mathcal{U}_{c}^{\gamma}\left(\mathcal{S}_{1}, \mathcal{S}_{0}\right)} E(\mathcal{O})
$$

Prova: Considere o conjunto de todos os vértices $t \in \mathcal{O}$ tal que existe um disco $\mathcal{V}_{\odot}(t, \gamma) \subseteq \mathcal{O}$, definido por:

$$
\boldsymbol{R}^{\gamma}(\mathcal{O})=\left\{t \in \mathcal{O} \mid \mathcal{V}_{\odot}(t, \gamma) \subseteq \mathcal{O}\right\}
$$

e o conjunto de todos os objetos $\gamma$-conexos possíveis da segmentação a partir das sementes $\mathcal{S}_{1}$ e $\mathcal{S}_{\mathbf{0}}$, definido por:

$$
\mathcal{U}_{c}^{\gamma}\left(\mathcal{S}_{1}, \mathcal{S}_{0}\right)=\left\{\mathcal{O} \in \mathcal{U}\left(\mathcal{S}_{1}, \mathcal{S}_{0}\right) \mid \mathcal{O} \text { é } \gamma \text {-conexo em relação a } \mathcal{S}_{1}\right\}
$$

Seja $\mathcal{O}_{\text {opt }}^{c}$ um objeto ótimo com energia máxima em $\mathcal{U}_{\boldsymbol{c}}^{\gamma}\left(\mathcal{S}_{1}, \mathcal{S}_{\mathbf{0}}\right)$. Então se cumpre que existe um componente conexo $C_{k}$ em $R^{\gamma}\left(\mathcal{O}_{\text {opt }}^{\boldsymbol{c}}\right)$, tal que $\mathcal{S}_{1} \subseteq C_{k}$. Como $C_{k}$ é conexo temos que existe um conjunto de vértices $\mathcal{S}_{1}^{c}$ compondo um caminho que interliga sementes em $\mathcal{S}_{1}$, tal que $\mathcal{S}_{1}^{c} \subseteq C_{k}$.

Assim, podemos deduzir que existe um conjunto de vértices $\mathcal{S}_{1}^{\gamma c}$ composto por discos $\mathcal{V}_{\odot}(t, \gamma)$, definido por:

$$
\mathcal{S}_{1}^{\gamma c}=\bigcup_{t \in \mathcal{S}_{1}^{c}} \mathcal{V}_{\odot}(t, \gamma)
$$

Como $\mathcal{S}_{1} \subseteq \mathcal{S}_{1}^{c} \subseteq \mathcal{S}_{1}^{\gamma c} \subseteq \mathcal{O}_{o p t}^{c}$ temos que $\mathcal{U}\left(\mathcal{O}_{o p t}^{c}, \mathcal{S}_{0}\right) \subseteq \mathcal{U}\left(\mathcal{S}_{1}^{\gamma c}, \mathcal{S}_{0}\right) \subseteq \mathcal{U}\left(\mathcal{S}_{1}^{c}, \mathcal{S}_{0}\right) \subseteq$ $\mathcal{U}\left(\mathcal{S}_{1}, \mathcal{S}_{0}\right)$. Assim, podemos concluir que:

$$
E\left(\mathcal{O}_{\text {opt }}^{c}\right)=\max _{\mathcal{O} \in \mathcal{U}_{c}^{\gamma}\left(\mathcal{S}_{1}, \mathcal{S}_{0}\right)} E(\mathcal{O})=\max _{\mathcal{O} \in \mathcal{U}\left(\mathcal{S}_{1}^{\gamma c}, \mathcal{S}_{0}\right)} E(\mathcal{O})
$$


e da Proposição 2 (pág. 44) podemos deduzir que

$$
E\left(\mathcal{O}_{o p t}^{c}\right)=E_{\mathcal{S}_{1}^{\gamma c}}=\min _{p \in \mathcal{S}_{1}^{\gamma c}} E_{\{p\}}
$$

Dado que $E_{\mathcal{V}_{\odot}(t, \gamma)}=\min _{p \in \mathcal{V}_{\odot}(t, \gamma)} E_{\{p\}}$ pode-se concluir que

$$
E\left(\mathcal{O}_{o p t}^{c}\right)=E_{\mathcal{S}_{1}^{\gamma c}}=\min _{\mathcal{V}_{\odot}(t, \gamma) \subseteq \mathcal{S}_{1}^{\gamma c}} E_{\mathcal{V}_{\odot}(t, \gamma)}
$$

o que equivale a

$$
E\left(\mathcal{O}_{o p t}^{c}\right)=E_{\mathcal{S}_{1}^{\gamma c}}=\min _{t \in \mathcal{S}_{1}^{c}} E_{\mathcal{V}_{\odot}(t, \gamma)}
$$

uma vez que para todo $t \in \mathcal{S}_{1}^{c}$ temos que $\mathcal{V}_{\odot}(t, \gamma) \subseteq \mathcal{S}_{1}^{\gamma c}$.

Portanto, um conjunto de sementes interligadas $\mathcal{S}_{1}^{c}$ passando por dentro do componente conexo $C_{k} \subseteq \boldsymbol{R}^{\gamma}\left(\mathcal{O}_{\text {opt }}^{c}\right)$ é suficiente para garantir um resultado ótimo, porém como $\mathcal{O}_{\text {opt }}^{\boldsymbol{c}}$ é desconhecido, devemos selecionar as sementes de modo a maximizar $\min _{t \in \mathcal{S}_{1}^{c}} E_{\mathcal{V}_{\odot}(t, \gamma)}$. Dado que $\mathcal{S}_{1} \subseteq \mathcal{S}_{1}^{c}$ é fixo, devemos mais precisamente selecionar $\mathcal{S}_{1}^{c} \backslash \mathcal{S}_{1}$ de modo a maximizar $\min _{t \in \mathcal{S}_{1}^{c} \backslash \mathcal{S}_{1}} E_{\mathcal{V}_{\odot}(t, \gamma)}$. Essa seleção é garantida pelo cálculo das interconexões pela função do riverbed, conforme explicado abaixo.

Para isso, vamos verificar os passos do Algoritmo 4 (pág. 50).

Primeiro, nas Linhas 2-3, o algoritmo da COIFT (Algoritmo 4) calcula o mapa de energias $\mathcal{E}_{\odot}$, tal que $\mathcal{E}_{\odot}(t)=E_{\mathcal{V}_{\odot}(t, \gamma)}$ para todo $t \in \mathcal{I}$, o que requer a erosão do mapa de custo calculado pelo algoritmo da IFT com $f_{\max }^{\sharp \mathcal{S}_{0}}$ usando só as sementes em $\mathcal{S}_{\mathbf{0}}$ para inicializar os custos dos caminhos triviais (Linha 1).

Logo, na Linha 4, o algoritmo da COIFT (Algoritmo 4) com o algoritmo NewDiskObjSeeds (Algoritmo 5, pág. 50) interconecta sementes não conexas em $\mathcal{S}_{1}$ através de vértices com valores altos de energia $E_{\mathcal{V}_{\odot}(t, \gamma)}$, fazendo uso da função de custo $f_{\text {river }}^{\prime}$ (Equação 4.13), tal que $\omega_{v}(t)=$ $K_{2}-E_{\mathcal{V}_{\odot}(t, \gamma)}$ e $K_{2}=\max _{t \in \mathcal{I}} E_{\mathcal{V}_{\odot}(t, \gamma)}$

Assim, um caminho riverbed $\tau_{r \sim t}$ (caminho calculado pelo Algoritmo 1 (pág. 27) usando $f_{\text {river }}^{\prime}$ ) a partir do vértice $r=s^{*}$ até $t$, sempre atravessa uma sequência de vértices intermediários $t_{i}$, para os quais o valor máximo de $\omega_{v}\left(t_{i}\right)=K_{2}-E_{\mathcal{V}_{\odot}\left(\boldsymbol{t}_{\boldsymbol{i}}, \gamma\right)}$ é minimizado ao longo de qualquer parte dele entre outros caminhos alternativos possíveis [Miranda et al. (2012)]. Consequentemente, um caminho riverbed pela $f_{\text {river }}^{\prime}$ sempre busca níveis mais altos de energia $E_{\mathcal{V}_{\odot}(t, \gamma)}$ que garante um corte ótimo como desejado.

Na Linha 1 do Algoritmo 5 (pág. 50), um vértice arbitrário em $\mathcal{S}_{1}$ é selecionado para ser usado como $s^{*}$ para o cálculo da IFT com função de custo $f_{\text {river }}^{\prime}$, gerando uma floresta de caminhos $P_{\text {river }}$, tal que para todo $t \in \mathcal{S}_{1}$ são selecionados todos os pixels $t_{i}$ no caminho $\pi_{t}^{P_{\text {river }}}$ conjuntamente com os vértices que compõem o seu disco $\mathcal{V}_{\odot}\left(t_{i}, \gamma\right)$ para compor um novo conjunto conexo de sementes internas $\mathcal{S}_{1}^{\gamma c}$ que satisfazem a restrição de $\gamma$-conexidade (Linha 4 do Algoritmo 4, pág. 50).

Finalmente, na Linha 5, a COIFT calcula o método da OIFT com $f^{0^{7}}$ (Equação 3.19) a partir dos conjuntos de sementes $\mathcal{S}_{1}^{\gamma \boldsymbol{c}}$ e $\mathcal{S}_{\mathbf{0}}$, gerando um resultado final, o qual é garantido ser um objeto $\gamma$-conexo $\mathcal{O}_{c}^{\gamma}$, tal que

$$
E\left(\mathcal{O}_{c}^{\gamma}\right)=\max _{\mathcal{O} \in \mathcal{U}\left(\mathcal{S}_{1}^{\gamma c}, \mathcal{S}_{0}\right)} E(\mathcal{O})=\max _{\mathcal{O} \in \mathcal{U}_{c}^{\gamma}\left(\mathcal{S}_{1}^{\gamma c}, \mathcal{S}_{0}\right)} E(\mathcal{O})=\max _{\mathcal{O} \in \mathcal{U}_{c}^{\gamma}\left(\mathcal{S}_{1}, \mathcal{S}_{0}\right)} E(\mathcal{O})
$$


PROVA DO TEOREMA DA OTIMALIDADE DO CORTE PELA COIFT COM LARGURA AJUSTÁVEL 
APÊNDICE D 


\section{Apêndice E}

\section{Prova do teorema da otimalidade do corte da IFT com $f_{\omega_{v}}$}

Com o fim de provar o Teorema 4 (pág. 72), precisamos dos Lemas 1, 2 e 3, nos quais usamos $F(\pi)$ para denotar o custo máximo ao longo de um caminho:

$$
F\left(\pi=\left\langle t_{1}, \ldots, t_{n}\right\rangle\right)=\max _{i=1,2, \ldots, n}\left\{f\left(\left\langle t_{1}, \ldots, t_{i}\right\rangle\right)\right\}
$$

Lema 1 Seja $P$ um mapa de predecessores calculado pelo algoritmo da IFT (Algoritmo 1). Para quaisquer dois caminhos $\delta_{t}^{P}=\left\langle t_{1}, t_{2}, \ldots, t_{n}=t\right\rangle$ e $\tau_{s}^{P}=\left\langle s_{1}, s_{2}, \ldots, s_{m}=s\right\rangle$, definidos por $P$, se $F\left(\delta_{t}^{P}\right)<F\left(\tau_{s}^{P}\right)$, então temos que o vértice $t$ foi removido de $\mathcal{Q}$ antes do vértice $s$ na Linha 8 do Algoritmo 1.

Prova: Seja $s_{k}$ um vértice em $\tau_{s}^{P}$, tal que $f\left(\left\langle s_{1}, \ldots, s_{k}\right\rangle\right)=F\left(\tau_{s}^{P}\right)$. Da Equação E.1, nós temos que $f\left(\left\langle t_{1}, \ldots, t_{i}\right\rangle\right) \leq F\left(\delta_{t}^{P}\right), i=1,2, \ldots, n$. A partir das premissas do Lema 1, nós podemos concluir que $F\left(\delta_{t}^{P}\right)<f\left(\left\langle s_{1}, \ldots, s_{k}\right\rangle\right)$. Assim, $f\left(\left\langle t_{1}, \ldots, t_{i}\right\rangle\right)<f\left(\left\langle s_{1}, \ldots, s_{k}\right\rangle\right), i=1,2, \ldots, n$.

A partir da dinâmica de execução do algoritmo da IFT (Algoritmo 1, pág. 27), sabemos que os caminhos $\delta_{t}^{P}$ e $\tau_{s}^{P}$ armazenados no mapa de predecessores $P$ são gradualmente calculados pela remoção dos vértices com custo mínimo em $\mathcal{Q}$ (Linha 8). Depois que $s_{k}$ é inserido na fila de prioridade $\mathcal{Q}$ com custo $V\left(s_{k}\right)=f\left(\left\langle s_{1}, \ldots, s_{k}\right\rangle\right)$, ele não será removido de $\mathcal{Q}$ antes que todos os vértices $t_{i}, i=1,2, \ldots, n$, sejam processados consecutivamente em $\mathcal{Q}$, com custos mais baixos $V\left(t_{i}\right)=f\left(\left\langle t_{1}, \ldots, t_{i}\right\rangle\right)$. Portanto, nós temos que $t=t_{n}$ é removido de $\mathcal{Q}$ antes do que $s$.

Do Lema 1, também podemos concluir o seguinte lema:

Lema 2 Seja $P$ um mapa de predecessores calculado pelo algoritmo da IFT (Algoritmo 1) com política de desempate FIFO. Para quaisquer dois caminhos $\delta_{s}^{P}$ e $\tau_{s^{\prime}}^{P}, s \neq s^{\prime}$, definidos em $P$, se $F\left(\tau_{s^{\prime}}^{P}\right)<F\left(\delta_{s}^{P}\right)$ e $f\left(\delta_{s}^{P} \cdot\langle s, t\rangle\right)=f\left(\tau_{s^{\prime}}^{P} \cdot\left\langle s^{\prime}, t\right\rangle\right)$, então nós temos que $\pi_{t}^{P} \neq \delta_{s}^{P} \cdot\langle s, t\rangle$.

Prova: O Algoritmo 1 (pág. 27) atribuirá $t$ ao primeiro caminho de melhor custo que o alcança, devido à desigualdade estrita na Linha 12. De acordo com o Lema 1 , nós temos que $s^{\prime}$ sai de $\mathcal{Q}$ antes de $s$. Consequentemente, o caminho $\tau_{s^{\prime}}^{P} \cdot\left\langle s^{\prime}, t\right\rangle$ é avaliado antes de $\delta_{s}^{P} \cdot\langle s, t\rangle$, oferecendo 
o mesmo custo (isso é, $f\left(\delta_{s}^{P} \cdot\langle s, t\rangle\right)=f\left(\tau_{s^{\prime}}^{P} \cdot\left\langle s^{\prime}, t\right\rangle\right)$ ). Portanto, nós temos que $\pi_{t}^{P}$ não pode ser $\delta_{s}^{P} \cdot\langle s, t\rangle$.

Lema 3 Considere o mapa $P$ calculado pelo algoritmo da IFT (Algoritmo 1) com $f_{\omega_{v}}$ em um digrafo simétrico fortemente conexo $G$. Para qualquer arco $\langle s, t\rangle \in \mathcal{A}$, tal que $\pi_{a \sim s}^{P}, \tau_{b \sim t}^{P}$ e $a \neq b$, temos que:

$$
\max \left\{F\left(\pi_{a \sim s}^{P}\right), F\left(\tau_{b \sim t}^{P}\right)\right\}=\max \left\{\omega_{v}(s), \omega_{v}(t)\right\} .
$$

\section{Prova:}

- Primeiramente, vamos provar que para $F\left(\pi_{a \sim s}^{P}\right)>F\left(\tau_{b \sim t}^{P}\right)$ temos que $F\left(\pi_{a \sim s}^{P}\right)=\omega_{v}(s)$. Isso pode ser provado por contradição.

Assuma que $F\left(\pi_{a \sim s}^{P}\right)>\omega_{v}(s)$ quando $F\left(\pi_{a \sim s}^{P}\right)>F\left(\tau_{b \sim t}^{P}\right)$. Assim, pelas Equações 5.1 e E.1 temos que

$$
F\left(\pi_{a \sim s}^{P}\right)=\max \left\{F\left(\pi_{a \sim P(s)}^{P}\right), f_{\omega_{v}}\left(\pi_{a \sim s}^{P}\right)\right\}=F\left(\pi_{a \sim P(s)}^{P}\right),
$$

uma vez que $f_{\omega_{v}}\left(\pi_{a \sim s}^{P}\right)=\omega_{v}(s)$.

Como

$$
F\left(\pi_{a \sim P(s)}^{P}\right)>F\left(\tau_{b \sim t}^{P}\right) \quad \text { e } \quad f_{\omega_{v}}\left(\pi_{a \sim s}^{P}\right)=f_{\omega_{v}}\left(\tau_{b \sim t}^{P} \cdot\langle t, s\rangle\right)=\omega_{v}(s)
$$

pelo Lema 2 nós temos que o caminho $\pi_{a \leadsto s}^{P}$ é inválido, levando a uma contradição.

- Devido ao problema simétrico, por argumentos similares, nós temos que se $F\left(\tau_{b \sim t}^{P}\right)>F\left(\pi_{a \sim s}^{P}\right)$ então $F\left(\tau_{b \sim t}^{P}\right)=\omega_{v}(t)$.

- $\mathrm{O}$ único caso ainda não tratado é quando $F\left(\pi_{a \sim s}^{P}\right)=F\left(\tau_{b \sim t}^{P}\right)$. Seja $C$ esse valor, ou seja,

$$
C=F\left(\pi_{a \sim s}^{P}\right)=F\left(\tau_{b \sim t}^{P}\right) .
$$

Pelas Equações 5.1 e E.1, temos que

$$
F\left(\pi_{a \sim s}^{P}\right) \geq \omega_{v}(s) \quad \text { e } \quad F\left(\tau_{b \sim t}^{P}\right) \geq \omega_{v}(t) .
$$

Consequentemente, para $F\left(\pi_{a \sim s}^{P}\right)=F\left(\tau_{b \sim t}^{P}\right)=C$ temos

$$
C \geq \max \left\{\omega_{v}(s), \omega_{v}(t)\right\}
$$

No Algoritmo 1 (pág. 27), os vértices $p_{i}$ e $q_{j}$ em $\pi_{a \sim s}^{P}=\left\langle p_{1}=a, p_{2}, \ldots, p_{n}=s\right\rangle \mathrm{e}$ $\tau_{b \sim t}^{P}=\left\langle q_{1}=b, q_{2}, \ldots, q_{m}=t\right\rangle$, respectivamente, são removidos consecutivamente da fila de prioridade, para valores crescentes de $i$ e $j$, sempre processando primeiro um vértice definindo um caminho $\left(\left\langle p_{1}=a, \ldots, p_{i}\right\rangle\right.$ ou $\left.\left\langle q_{1}=b, \ldots, q_{j}\right\rangle\right)$ com custo mais baixo $\left(\omega_{v}\left(p_{i}\right)\right.$ ou $\left.\omega_{v}\left(q_{j}\right)\right)$. 
Assim, quando

$$
\left\{p_{i}, q_{j}\right\} \subset \mathcal{Q} \quad \text { e } f_{\omega_{v}}\left(\left\langle p_{1}=a, \ldots, p_{i}\right\rangle\right)=\omega_{v}\left(p_{i}\right)=C,
$$

o vértice $p_{i}$ somente pode ser removido da fila de prioridade $\mathcal{Q}$, se $f_{\omega_{v}}\left(\left\langle q_{1}=b, \ldots, q_{j}\right\rangle\right)=\omega_{v}\left(q_{j}\right)=C$, e vice-versa.

Consequentemente, na última iteração, teremos um vértice em $\mathcal{Q}$ com custo igual a $C$ (isso é, $f_{\omega_{v}}\left(\pi_{a \sim s}^{P}\right)=\omega_{v}(s)=C$ ou $\left.f_{\omega_{v}}\left(\tau_{b \sim t}^{P}\right)=\omega_{v}(t)=C\right)$. Portanto, $\max \left\{\omega_{v}(s), \omega_{v}(t)\right\}=C$.

Assim, o Teorema 4 pode ser provado por contradição como se segue.

Teorema 4 (Otimalidade do Corte da IFT com $f_{\omega_{v}}$ ) Para um digrafo simétrico fortemente conexo $G$ com dois conjuntos de sementes $\mathcal{S}_{0}$ e $\mathcal{S}_{1}$ dados, denota-se $\mathcal{U}\left(\mathcal{S}_{1}, \mathcal{S}_{0}\right)=\left\{\mathcal{O} \subseteq \mathcal{I} \mid \mathcal{S}_{1} \subseteq \mathcal{O} \subseteq \mathcal{I} \backslash \mathcal{S}_{0}\right\}$ como o universo de todos os objetos possíveis satisfazendo as restrições de semente. O algoritmo da IFT (Algoritmo 1) em $G$ com função de custo de caminho $f_{\omega_{v}}$ e $\mathcal{S}=\mathcal{S}_{0} \cup \mathcal{S}_{1}$ produz um objeto segmentado $\mathcal{O}^{P}$ o qual maximiza a energia $E(\mathcal{O})$ (Equação 5.2) entre todos os objetos em $\mathcal{U}\left(\mathcal{S}_{1}, \mathcal{S}_{0}\right)$. Isso é, $E\left(\mathcal{O}^{P}\right)=\max _{\mathcal{O} \in \mathcal{U}\left(\mathcal{S}_{1}, \mathcal{S}_{0}\right)} E(\mathcal{O})$.

Prova: Seja $E_{\text {opt }}$ o valor ótimo de energia, isso é,

$$
E_{\text {opt }}=\max _{\mathcal{O} \in \mathcal{U}\left(\mathcal{S}_{1}, \mathcal{S}_{0}\right)} E(\mathcal{O})
$$

Assuma que

$$
E\left(\mathcal{O}^{P}\right)<E_{\text {opt }}=E\left(\mathcal{O}_{\text {opt }}\right)
$$

onde $\mathcal{O}_{\text {opt }}$ denota uma solução ótima. Seja $\langle s, t\rangle$ um arco em $\mathcal{C}\left(\mathcal{O}^{P}\right)$, tal que

$$
E\left(\mathcal{O}^{P}\right)=\max \left\{\omega_{v}(s), \omega_{v}(t)\right\}
$$

Como

$$
\max \left\{\omega_{v}(s), \omega_{v}(t)\right\}=E\left(\mathcal{O}^{P}\right)<E\left(\mathcal{O}_{\text {opt }}\right)
$$

pela Equação 5.2 podemos concluir que

$$
\langle s, t\rangle \notin \mathcal{C}\left(\mathcal{O}_{\text {opt }}\right) .
$$

Note também que $\langle t, s\rangle \notin \mathcal{C}\left(\mathcal{O}_{\text {opt }}\right)$ pelos mesmos motivos. Assim temos duas possibilidades, $\{s, t\} \subset \mathcal{O}_{\text {opt }}$ ou $\{s, t\} \subset \mathcal{I} \backslash \mathcal{O}_{\text {opt }}$ :

1. No primeiro caso (Figura E.1a), $\pi_{\mathcal{S}_{0} \sim t}^{P}$ necessariamente passa através de um arco $\langle y, x\rangle$, tal 
que $\langle x, y\rangle \in \mathcal{C}\left(\mathcal{O}_{\text {opt }}\right)$ e pela Equação 5.2 temos

$$
\max \left\{\omega_{v}(x), \omega_{v}(y)\right\} \geq \min _{\langle a, b) \in \mathcal{C}\left(\mathcal{O}_{o p t}\right)}\left\{\max \left\{\omega_{v}(a), \omega_{v}(b)\right\}\right\}=E_{\text {opt }}
$$

Consequentemente, temos

$$
F\left(\pi_{\mathcal{S}_{0} \sim t}^{P}\right) \geq E_{\text {opt }}
$$

2. No segundo caso (Figura E.1b), $\tau_{\mathcal{S}_{1} \sim s}^{P}$ passa através de um arco $\left\langle x^{\prime}, y^{\prime}\right\rangle$, tal que $\left\langle x^{\prime}, y^{\prime}\right\rangle \in \mathcal{C}\left(\mathcal{O}_{\text {opt }}\right)$ e pela Equação 5.2 temos

$$
\max \left\{\omega_{v}\left(x^{\prime}\right), \omega_{v}\left(y^{\prime}\right)\right\} \geq E_{\text {opt }},
$$

o qual implica que

$$
F\left(\tau_{\mathcal{S}_{1} \sim s}^{P}\right) \geq E_{\text {opt }}
$$

Portanto, em ambos casos temos

$$
\max \left\{F\left(\pi_{\mathcal{S}_{0} \sim t}^{P}\right), F\left(\tau_{\mathcal{S}_{1} \sim s}^{P}\right)\right\} \geq E_{\text {opt }}
$$

Mas, pelo Lema 3 temos

$$
\max \left\{F\left(\pi_{\mathcal{S}_{0} \sim t}^{P}\right), F\left(\tau_{\mathcal{S}_{1} \sim s}^{P}\right)\right\}=\max \left\{\omega_{v}(s), \omega_{v}(t)\right\}=E\left(\mathcal{O}^{P}\right)
$$

levando a uma contradição.

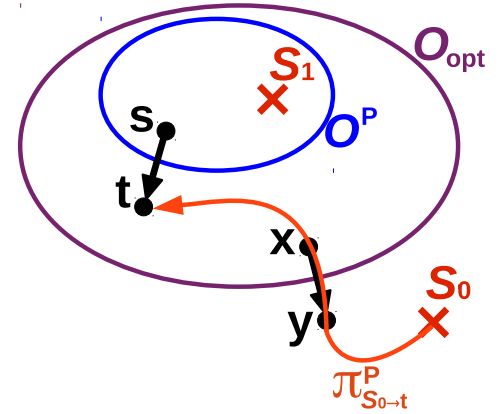

(a)

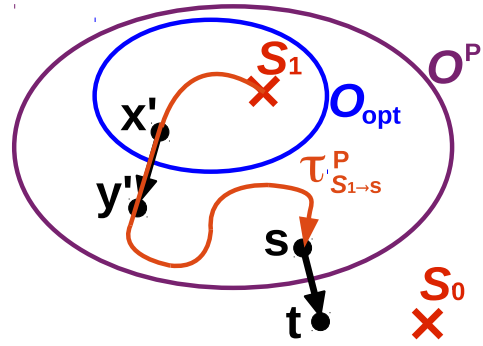

(b)

Figura E.1: Representação esquemática para a prova de corretude do Teorema 4. $\mathcal{O}_{\text {opt }}$ é o objeto que representa a solução ótima, $\mathcal{O}^{P}$ representa o objeto segmentado definido pela floresta $P$ calculada pelo Algoritmo $1, \mathcal{S}=\left\{\mathcal{S}_{0}, \mathcal{S}_{1}\right\}$, e $\langle s, t\rangle$ é um arco em $\mathcal{C}\left(\mathcal{O}^{P}\right)$ sendo que $\langle s, t\rangle \notin \mathcal{O}_{\text {opt }}$. Assim temos duas possibilidades: (a) O primeiro caso: $\{s, t\} \subset \mathcal{O}_{\text {opt }}$. (b) O segundo caso: $\{s, t\} \subset \mathcal{I} \backslash \mathcal{O}_{\text {opt }}$. 


\section{Referências Bibliográficas}

Ahuja et al. (1993) R.K. Ahuja, T.L. Magnanti e J.B. Orlin. Network Flows: Theory, Algorithms and Applications. Prentice-Hall. Citado na pág. 27

Bai e Sapiro (2007) X. Bai e G. Sapiro. Distance cut: interactive segmentation and matting of images and videos. Em IEEE International Conference on Image Processing (ICIP), volume 2, páginas II - 249-II - 252, San Antonio, Texas. Citado na pág. 1, 21, 36

Barreto Alexandre et al. (2015) E. Barreto Alexandre, A. Shankar Chowdhury, A.X. Falcão e P.A.V. Miranda. Ift-slic: A general framework for superpixel generation based on simple linear iterative clustering and image foresting transform. Em Graphics, Patterns and Images (SIBGRAPI), 2015 28th SIBGRAPI Conference on, páginas 337-344. doi: 10.1109/SIBGRAPI.2015.20. Citado na pág. 24

Bejar e Miranda (2015) Hans H.C. Bejar e Paulo A.V. Miranda. Oriented relative fuzzy connectedness: Theory, algorithms, and its applications in hybrid image segmentation methods. EURASIP Journal on Image and Video Processing, 2015(21). doi: 10.1186/s13640-015-0067-4. Citado na pág. $45,60,64$

Boykov e Funka-Lea (2006) Y. Boykov e G. Funka-Lea. Graph cuts and efficient N-D image segmentation. International Journal of Computer Vision, 70(2):109-131. ISSN 0920-5691. doi: http://dx.doi.org/10.1007/s11263-006-7934-5. Citado na pág. 1, 2, 21, 36

Ciesielski e Udupa (2010) K.C. Ciesielski e J.K. Udupa. Affinity functions in fuzzy connectedness based image segmentation i: Equivalence of affinities. Computer Vision and Image Understanding, 114(1):146-154. ISSN 1077-3142. Citado na pág. 38

Ciesielski et al. (2007) K.C. Ciesielski, J.K. Udupa, P.K. Saha e Y. Zhuge. Iterative relative fuzzy connectedness for multiple objects with multiple seeds. Computer Vision and Image Understanding, 107(3):160-182. ISSN 1077-3142. Citado na pág. 1, 2, 21, 36, 60

Ciesielski et al. (2011) K.C. Ciesielski, J.K. Udupa, A.X. Falcão e P.A.V. Miranda. Comparison of fuzzy connectedness and graph cut segmentation algorithms. Em Proceedings of SPIE on Medical Imaging: Image Processing, volume 7962, Orlando. doi: 10.1117/12.872522. Citado na pág. 30

Ciesielski et al. (2012a) K.C. Ciesielski, J.K. Udupa, A.X. Falcão e P.A.V. Miranda. Fuzzy connectedness image segmentation in graph cut formulation: A linear-time algorithm and a comparative analysis. Journal of Mathematical Imaging and Vision, 44(3):375-398. Citado na pág. 36

Ciesielski et al. (2012b) K.C. Ciesielski, J.K. Udupa, A.X. Falcão e P.A.V. Miranda. A unifying graph-cut image segmentation framework: algorithms it encompasses and equivalences among them. Em Proc. of SPIE on Medical Imaging: Image Processing, volume 8314. doi: 10.1117/12. 911810. Citado na pág. 1, 36

Ciesielski et al. (2018) K.C. Ciesielski, A.X. Falcão e P.A.V. Miranda. Path-value functions for which dijkstra's algorithm returns optimal mapping. Journal of Mathematical Imaging and Vision. ISSN 1573-7683. doi: 10.1007/s10851-018-0793-1. URL https://doi.org/10.1007/s10851-018-0793-1. Citado na pág. 32, 34, 35 
Ciesielski et al. (2013) Krzysztof Chris Ciesielski, P.A.V. Miranda, A.X. Falcão e Jayaram K. Udupa. Joint graph cut and relative fuzzy connectedness image segmentation algorithm. Medical Image Analysis (MEDIA), 17(8):1046-1057. Citado na pág. 60, 64

Condori et al. (2017) M.A.T. Condori, L.A.C. Mansilla e P.A.V. Miranda. Bandeirantes: A graph-based approach for curve tracing and boundary tracking. Em Mathematical Morphology and Its Applications to Signal and Image Processing, volume vol. LNCS 10225, páginas 95-106, Fontainebleau, France. Springer International Publishing. ISBN 978-3-319-57240-6. Citado na pág. $4,32,35$

Couprie et al. (2010) C. Couprie, L. Grady, L. Najman e H. Talbot. Power watersheds: A unifying graph-based optimization framework. Transactions on Pattern Analysis and Machine Intelligence, 99. ISSN 0162-8828. doi: http://doi.ieeecomputersociety.org/10.1109/TPAMI.2010.200. Citado na pág. $1,21,36$

Cousty et al. (2010) J. Cousty, G. Bertrand, L. Najman e M. Couprie. Watershed cuts: Thinnings, shortest path forests, and topological watersheds. IEEE Transactions on Pattern Analysis and Machine Intelligence, 32:925-939. ISSN 0162-8828. Citado na pág. 1, 2, 21, 36

de Moraes Braz e Miranda (2014) C. de Moraes Braz e P.A.V. Miranda. Image segmentation by image foresting transform with geodesic band constraints. Em Image Processing (ICIP), 2014 IEEE International Conference on, páginas 4333-4337. doi: 10.1109/ICIP.2014.7025880. Citado na pág. 4

Dice (1945) L.R. Dice. Measures of the amount of ecologic association between species. Ecology, 26:297-302. Citado na pág. 15

Dijkstra (1959) E.W. Dijkstra. A note on two problems in connexion with graphs. Numerische Mathematik, 1:269-271. Citado na pág. 27

Falcão e Bergo (2004) A.X. Falcão e F.P.G. Bergo. Interactive volume segmentation with differential image foresting transforms. IEEE Transactions on Medical Imaging, 23(9):1100-1108. Citado na pág. 21

Falcão e da Cunha (2001) A.X. Falcão e B.S. da Cunha. Multiscale Shape Representation by Image Foresting Transform. Em Milan Sonka e Kenneth Hanson, editors, Proceedings of SPIE on Medical Imaging, volume 4322, páginas 1091-1100, San Diego, CA. Citado na pág. 21

Falcão et al. (1998) A.X. Falcão, J.K. Udupa, S. Samarasekera, S. Sharma, B.E. Hirsch e R.A. Lotufo. User-steered image segmentation paradigms: Live-wire and live-lane. Graphical Models and Image Processing, 60(4):233-260. Citado na pág. 1

Falcão et al. (2000) A.X. Falcão, J.K. Udupa e F.K. Miyazawa. An ultra-fast user-steered image segmentation paradigm: Live-wire-on-the-fly. IEEE Transactions on Medical Imaging, 19(1):5562. Citado na pág. 1,28

Falcão et al. (2001) A.X. Falcão, B.S. da Cunha e R.A. Lotufo. Design of connected operators using the image foresting transform. Em SPIE on Medical Imaging, volume 4322, páginas 468479. Citado na pág. 21

Falcão et al. (2002) A.X. Falcão, L.F. Costa e B.S. da Cunha. Multiscale skeletons by image foresting transform and its applications to neuromorphometry. Pattern Recognition, 35(7):15711582. Citado na pág. 24

Falcão et al. (2004) A.X. Falcão, J. Stolfi e R.A. Lotufo. The image foresting transform: Theory, algorithms, and applications. IEEE Transactions on Pattern Analysis and Machine Intelligence, 26(1):19-29. Citado na pág. 1, 3, 4, 28, 32, 34, 40, 71, 73 
Grady (2006) L. Grady. Random walks for image segmentation. IEEE Transactions on Pattern Analysis and Machine Intelligence, 28(11):1768-1783. ISSN 0162-8828. Citado na pág. 1, 21, 36

Gulshan et al. (2010) V. Gulshan, C. Rother, A. Criminisi, A. Blake e A. Zisserman. Geodesic star convexity for interactive image segmentation. Em Proceedings of Computer Vision and Pattern Recognition, páginas 3129-3136. Citado na pág. 59

Hellden (1980) U. Hellden. A test of landsat-2 imagery and digital data for thematic mapping illustrated by an environmental study in northern kenya. Relatório Técnico 47, Lund University, Natural Geography Institute, Sweden. Citado na pág. 15

Herman e Carvalho (2001) G.T. Herman e B.M. Carvalho. Multiseeded segmentation using fuzzy connectedness. IEEE Transactions on Pattern Analysis and Machine Intelligence, 23:460474. Citado na pág. 24

Labatut e Cherifi (2012) V. Labatut e H. Cherifi. Accuracy measures for the comparison of classifiers. CoRR, abs/1207.3790. Citado na pág. 15

Lézoray e Grady (2012) O. Lézoray e L. Grady. Image Processing and Analysis with Graphs: Theory and Practice. CRC Press, California, USA. ISBN 1439855072. Citado na pág. 1, 2, 3

Lotufo e Falcão (2000) R.A. Lotufo e A.X. Falcão. The ordered queue and the optimality of the watershed approaches. Em Mathematical Morphology and its Applications to Image and Signal Processing, volume 18, páginas 341-350. Kluwer. Citado na pág. 72

Madabhushi e Udupa (2005) A. Madabhushi e J.K. Udupa. Interplay between intensity standardization and inhomogeneity correction in mr image processing. IEEE TMI, 24(5):561-576. Citado na pág. 1

Mansilla e Miranda (2013a) L.A.C. Mansilla e P.A.V. Miranda. Image segmentation by oriented image foresting transform: Handling ties and colored images. Em 18th International Conference on Digital Signal Processing (DSP), páginas 1-6, Santorini, Greece. IEEE. Citado na pág. 24, 38, 41

Mansilla e Miranda (2013b) L.A.C. Mansilla e P.A.V. Miranda. Image segmentation by oriented image foresting transform with geodesic star convexity. Em Computer Analysis of Images and Patterns (CAIP), volume 8047, páginas 572-579, York, UK. Citado na pág. 24

Mansilla e Miranda (2016) L.A.C. Mansilla e P.A.V. Miranda. Oriented image foresting transform segmentation: Connectivity constraints with adjustable width. Em Graphics, Patterns and Images (SIBGRAPI), 2016 29th SIBGRAPI Conference on, páginas 289-296, São José Dos Campos, SP, Brazil. doi: 10.1109/SIBGRAPI.2016.047. Citado na pág. 4, 41

Mansilla et al. (2013a) L.A.C. Mansilla, F.A.M. Cappabianco e P.A.V. Miranda. Image segmentation by image foresting transform with non-smooth connectivity functions. Em XXVI Conference on Graphics, Patterns and Images (SIBGRAPI), páginas 147-154, Arequipa, Perú. IEEE. Citado na pág. 4,24

Mansilla et al. (2013b) L.A.C. Mansilla, M.P. Jackowski e P.A.V. Miranda. Image foresting transform with geodesic star convexity for interactive image segmentation. Em IEEE International Conference on Image Processing (ICIP), páginas 4054-4058, Melbourne, Australia. Citado na pág. 4,38

Mansilla et al. (2016) L.A.C. Mansilla, P.A.V. Miranda e F.A.M. Cappabianco. Oriented image foresting transform segmentation with connectivity constraints. Em Image Processing (ICIP), 2016 23rd IEEE International Conference on, páginas 2554-2558, Phoenix, Arizona, USA. doi: 10.1109/ICIP.2016.7532820. Citado na pág. 4, 41, 73 
Miranda e Falcão (2009) P.A.V. Miranda e A.X. Falcão. Links between image segmentation based on optimum-path forest and minimum cut in graph. Journal of Mathematical Imaging and Vision. ISSN 1573-7683. doi: 10.1007/s10851-009-0159-9. Citado na pág. 25, 28, 36

Miranda e Mansilla (2014) P.A.V. Miranda e L.A.C. Mansilla. Oriented image foresting transform segmentation by seed competition. Image Processing, IEEE Transactions on, 23(1):389-398. ISSN 1057-7149. doi: 10.1109/TIP.2013.2288867. Citado na pág. 4, 24, 30, 38, 41, 60, 61, 63

Miranda et al. (2008) P.A.V. Miranda, A.X. Falcão, A. Rocha e F.P.G. Bergo. Object delineation by $\kappa$-connected components. EURASIP Journal on Advances in Signal Processing, páginas 1-14. Citado na pág. 24

Miranda et al. (2010) P.A.V. Miranda, A.X. Falcão e J.K. Udupa. Synergistic arc-weight estimation for interactive image segmentation using graphs. Computer Vision and Image Understanding, 114(1):85-99. ISSN 1077-3142. Citado na pág. 38, 60

Miranda et al. (2011) P.A.V. Miranda, A.X. Falcão e T.V. Spina. The riverbed approach for user-steered image segmentation. Em Proceedings of the International Conference on Image Processing, páginas 3133-3136, Brussels, Belgium. IEEE. doi: 10.1109/ICIP.2011.6116330. Citado na pág. 21,24

Miranda et al. (2012) P.A.V. Miranda, A.X. Falcão e T.V. Spina. Riverbed: A novel user-steered image segmentation method based on optimum boundary tracking. IEEE Transactions on Image Processing. doi:10.1109/TIP.2012.2188034. Citado na pág. 4, 24, 40, 71, 80, 84

Nowozin e Lampert (2010) Sebastian Nowozin e Christoph H. Lampert. Global interactions in random field models: A potential function ensuring connectedness. SIAM Journal on Imaging Sciences, 3(4):1048-1074. doi: 10.1137/090752614. URL http://dx.doi.org/10.1137/090752614. Citado na pág. $1,2,3$

Peng et al. (2011) Bo Peng, Lei Zhang, David Zhang e Jian Yang. Image segmentation by iterated region merging with localized graph cuts. Pattern Recognition, 44(10-11):2527-2538. Citado na pág. 1

Rauber et al. (2013) P.E. Rauber, A.X. Falcão, T.V. Spina e P.J. Rezende. Interactive segmentation by image foresting transform on superpixel graphs. Em XXVI Conference on Graphics, Patterns and Images (SIBGRAPI), páginas 131-138. IEEE. Citado na pág. 15

Sørensen (1948) T. Sørensen. A Method of Establishing Groups of Equal Amplitude in Plant Sociology Based on Similarity of Species Content and Ist Application to Analyses of the Vegetation on Danish Commons. Det Kongelige Danske Videnskabernes Selskab. Munksgaard. Citado na pág. 15

Spina et al. (2012) T.V. Spina, P.A.V. Miranda e A.X. Falcão. Intelligent understanding of user interaction in image segmentation. International Journal of Pattern Recognition and Artificial Intelligence (IJPRAI), 26(2). Citado na pág. 21

Stehman (1997) S.V. Stehman. Selecting and interpreting measures of thematic classification accuracy. Em Remote Sensing of Environment, volume 62, páginas 77-89. Citado na pág. 15

Strand et al. (2013) R. Strand, K.C. Ciesielski, F. Malmberg e P.K. Saha. The minimum barrier distance. Computer Vision and Image Understanding, 117:429-437. Citado na pág. 24

Torres et al. (2004) R.S. Torres, A.X. Falcão e L.F. Costa. A graph-based approach for multiscale shape analysis. Pattern Recognition, 37(6):1163-1174. Citado na pág. 24

van Rijsbergen (1979) C.J. van Rijsbergen. Information retrieval. Wiley Inter-science, London, second ed. Citado na pág. 15 
Vezhnevets e Konouchine (2005) V. Vezhnevets e V. Konouchine. "growcut- interactive multilabel N-D image segmentation by cellular automata. Em Proc. Graphicon., páginas 150-156. Citado na pág. 21

Vicente et al. (2008) S. Vicente, V. Kolmogorov e C. Rother. Graph cut based image segmentation with connectivity priors. Em IEEE Proc. of Computer Vision and Pattern Recognition (CVPR), páginas 1-8, Anchorage, Alaska. Citado na pág. 1, 2, 3, 21, 41

W.Yang et al. (2010) W.Yang, J.Cai, J. Zheng e J. Luo. User-friendly interactive image segmentation through unified combinatorial user inputs. IEEE Transactions on Image Processing, 19 (9):2470-2479. Citado na pág. 1

Zeng et al. (2008) Yun Zeng, Dimitris Samaras, Wei Chen e Qunsheng Peng. Topology cuts: A novel min-cut/max-flow algorithm for topology preserving segmentation in nâd images. Computer Vision and Image Understanding, 112(1):81 90. ISSN 1077-3142. doi: http://dx.doi.org/10.1016/j.cviu.2008.07.008. URL http://www.sciencedirect.com/science/article/pii/S1077314208001094. Special Issue on Discrete Optimization in Computer Vision. Citado na pág. 1, 2, 3, 41 